\title{
Deep Extragalactic Neutral Hydrogen Surveys with the Green Bank Telescope
}

Nickolas M Pingel

Follow this and additional works at: https://researchrepository.wvu.edu/etd

\section{Recommended Citation}

Pingel, Nickolas M, "Deep Extragalactic Neutral Hydrogen Surveys with the Green Bank Telescope" (2017). Graduate Theses, Dissertations, and Problem Reports. 7228.

https://researchrepository.wvu.edu/etd/7228

This Dissertation is protected by copyright and/or related rights. It has been brought to you by the The Research Repository @ WVU with permission from the rights-holder(s). You are free to use this Dissertation in any way that is permitted by the copyright and related rights legislation that applies to your use. For other uses you must obtain permission from the rights-holder(s) directly, unless additional rights are indicated by a Creative Commons license in the record and/ or on the work itself. This Dissertation has been accepted for inclusion in WVU Graduate Theses, Dissertations, and Problem Reports collection by an authorized administrator of The Research Repository @ WVU.

For more information, please contact researchrepository@mail.wvu.edu. 


\title{
Deep Extragalactic Neutral Hydrogen Surveys with the Green Bank Telescope \\ Nickolas M. Pingel
}

\author{
Dissertation Submitted to \\ The Eberly College of Arts and Sciences \\ at West Virginia University \\ in partial fulfillment of the requirements \\ for the degree of \\ Doctor of Philosophy \\ in \\ Physics \\ D.J. Pisano, Ph.D., Chair \\ Loren Anderson, Ph.D. \\ Kevin Bandura, Ph.D. \\ Duncan Lorimer, Ph.D. \\ Richard Prestage, Ph.D. \\ Morgantown, West Virginia, USA \\ 2017
}

Keywords: galaxies: evolution; galaxies: star formation; phased array feeds: observations; phased array feeds: beamforming

Copyright 2018 Nickolas Pingel 
Abstract

\title{
Deep Extragalactic Neutral Hydrogen Surveys with the Green Bank Telescope
}

\author{
Nickolas Pingel
}

The Green Bank Telescope (GBT) is utilized to map the galaxies of the Westerbork Radio Synthesis Telescope (WSRT) Hydrogen Accretion in LOcal GAlaxieS (HALOGAS) Survey to look for diffuse Hi features that were previously undetected due, in part, to the lack of angular sensitivity of the WSRT. Cosmological simulations predict that cold gas should accrete onto galaxies in the nearby Universe in the form of vast filaments that penetrated into the halo and extend down to the disk. While such filaments are too hot to be observed in Hi emission towards the edge of the virial radius, it is thought that the gas will cool near enough disk to be observed in Hi near the disk at Hi column densities on the order of $10^{18} \mathrm{~cm}^{-2}$.

We find no evidence for significant large scale Hi features that could be associated with the theorized cold flows in the HALOGAS sample of galaxies an Hi column density level of $10^{18} \mathrm{~cm}^{-2}$. This result suggests future $\mathrm{HI}$ surveys should invest the necessary time to probe down to Hi column densities $\sim 10^{17} \mathrm{~cm}^{-2}$ in order to detect HI associated with infalling cold flows and the cosmic web.

In addition to going down to rarely probed Hi column density levels, this work also presents the results of commissioning observations of a cryogenically cooled phased array feed for the GBT that is optimized for observations between one and two GHz. Maps of an external galaxy and faint Hi cloud compare well to data already obtained with the traditional single pixel feed, indicating this instrument will be a definite to the current single pixel feed horn. The ability of PAFs to simultaneously form multiple beams on the sky increases the field of view, and thus survey speeds, such that Hi column density levels below $10^{18} \mathrm{~cm}^{-2}$ can be reached in a realistic time constraints. Observing the Universe at these column density levels will reveal galaxy evolution mechanisms that so far have remained undetected. 


\section{Acknowledgements}

First and foremost, I wish to thank my Mom and Dad, Robin and Michael Pingel. Growing up as an only child meant that, for better or worse at times, their attention to my development was undivided. From a young age I was taught to approach every aspect of life - whether that be a sport, academics, or general interest — full heartedly. This dissertation is the ultimate collimation of that philosophy. I love and appreciate you both in ways that cannot be expressed in the few words alloted here. Thank you for your unwavering support.

To Carolyn, the love of my life, your support and love has and always will abide. We took a chance entering the adventure that was graduate school at West Virgina University and moving to Morgantown. We've cultivated an incredibly community both here and back in Madison. And while it will be difficult to leave them both temporarily, I am excited to build new communities with you wherever we go. I wish to be the same source of encouragement for the pursuit of your passions as you have provided me over the years. I can't wait to travel the world with you.

I of course also wish to thank my adviser, Dr. D. J. Pisano. Allowing me to begin research my first year of graduate school helped immensely in jump-starting my development as a scientist. Whenever I came to you with something to chat about - whether that be a concept I did not initially understand or an exciting result - you always made the time necessary time to guide me to the right interpretation on my own. I cannot stress enough how integral you were in helping me achieve my dream of becoming a professional astronomer. I look forward to working together for many years to come.

In terms of other influential advisers, I wish to also thank Dr. Snezana Stanimirović, who took a risk on sophomore at the University of Wisconsin-Madison with no astronomy background whatsoever. I am immensely proud of the work that we accomplished over the subsequent four years, and I am happy that it continued even into my time at WVU. Thank you for taking a chance on me.

To my friends that I grew up with, I realize it is particularly rare to have a friend group that stays close even as we spread out throughout the U.S. and even to Australia. I love you all. To my friends I made in Morgantown - and my band mates in [Indistinct Chattering] — you all helped to keep me sane and grounded while I was finishing up this Dissertation. I'll never forget playing at 123 Pleasant Street or just the nights watching movies and goofing around.

And I need to give one final thanks to my friends at Chestnut Brew Works, who gave me the fuel I needed to finish this process with a smile of my face. 


\section{Table of Contents}

List of Tables

List of Figures $\quad$ vii

1 Introduction 1

1.1 The Utility of Neutral Hydrogen . . . . . . . . . . . . . . . . . . . . . 1

1.2 Basic Observable Hi Properties . . . . . . . . . . . . . . . . . . . . . 2

1.3 Accretion of Hi Onto Galaxies . . . . . . . . . . . . . . . . . . 6

1.4 Spectral Line Observation with Phased Array Feeds . . . . . . . . . . 11

1.5 Sampling the Focal Plane . . . . . . . . . . . . . . . . . . 13

1.6 Dissertation Outline . . . . . . . . . . . . . . . 15

2 A Pilot GBT Survey of the HALOGAS Galaxies 16

2.1 Introduction . . . . . . . . . . . . . . . . . . . . 17

2.2 The HALOGAS Data and Sample . . . . . . . . . . . . . . 23

2.2.1 WSRT Data Cubes . . . . . . . . . . . . . . 23

2.2.2 Sample....................... 25

2.3 GBT Observations and Data Reduction and Low-Resolution WSRT

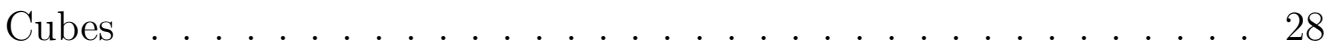

2.3.1 Observations and Data Reduction . . . . . . . . . . . . . 28

2.3.2 Convolution of WSRT data . . . . . . . . . . . . . . 32

2.3.3 Primary-beam Correction ....................... 36

2.4 Results . . . . . . . . . . . . . . . . . . . . 38

2.4.1 Summary of Analysis . . . . . . . . . . . . . . . 38

2.4.1.1 Global Hi Profiles and Noise . . . . . . . . . . . 38

2.4.1.2 $N_{\mathrm{HI}}$ Images . . . . . . . . . . . . . . . . . . 39

2.4.1.3 The Cumulative Hi Mass vs. $N_{\mathrm{HI}} \ldots \ldots . . . . . . .44$

2.4.1.4 Radial Profiles of $N_{\mathrm{HI}}$ and Cumulative Flux . . . . . 42

2.4.2 NGC891 ...................... . . . . 44

2.4.2.1 The Cumulative Hi Mass vs. $N_{\mathrm{HI}} \ldots \ldots . . . . . . .49$

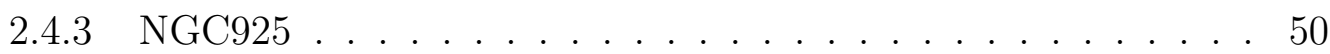

$2.4 .4 \mathrm{NGC4414}$...................... . . . 57

2.4 .5 NGC4565 ...................... . . . . 63

2.5 Discussion . . . . . . . . . . . . . . . . . . . 67

2.5.1 Implications for Future Hi Surveys . . . . . . . . . . . . . . . 68

2.5.2 The Relationship Between Diffuse Hi and Cold Mode Accretion 70

2.6 Conclusions and Future Work . . . . . . . . . . . . . . . . 79

3 The Results of the Full GBT-HALOGAS Survey 83

3.1 Introduction . . . . . . . . . . . . . . . . . 83

3.2 Alterations to Analysis Methods . . . . . . . . . . . . . . . 85

3.3 The Full GBT Survey Data and Sample . . . . . . . . . . . . . . . 92

3.3.1 NGC672 ........................ . . 92 
3.3.2 NGC1003 . . . . . . . . . . . . . . . . . . . . . . . . . . . . . 94

$3.3 .3 \quad$ UGC4278 $\ldots \ldots \ldots \ldots \ldots$

$3.3 .4 \quad \mathrm{NGC} 2541 \ldots \ldots \ldots \ldots$

$3.3 .5 \quad$ NGC4244 . . . . . . . . . . . . . . . . . . . . . . . . . . 98

$3.3 .6 \quad \mathrm{NGC} 4258 \ldots \ldots \ldots 0$

$3.3 .7 \quad$ NGC4559 . . . . . . . . . . . . . . . . . . . . . . . 102

$3.3 .8 \quad$ UGC7774 . . . . . . . . . . . . . . . . . . . . . . . . . . 102

$3.3 .9 \quad$ NGC4631 . . . . . . . . . . . . . . . . . . . . . . . . . . . . . . 108

3.3 .10 NGC5023 . . . . . . . . . . . . . . . . . . . . . . . . 109

3.3 .11 NGC5229 . . . . . . . . . . . . . . . . . . . . . 115

3.3 .12 NGC5585 . . . . . . . . . . . . . . . . . . . 116

3.4 Analysis . . . . . . . . . . . . . . . . . . . . 116

3.4.1 General Trends . . . . . . . . . . . . . . . . . . . . 118

3.4 .2 The Diffuse Hi Mass Fraction . . . . . . . . . . . . . . . . . 132

3.5 The Dearth of Diffuse Hi _ . . . . . . . . . . . . . . . . . . 134

3.6 Conclusions . . . . . . . . . . . . . . . . . . 136

4 Neutral Hydrogen Observations with the Focal L-Band Array for the Green

Bank Telescope (FLAG) 138

4.1 Introduction . . . . . . . . . . . . . . . . . . . 138

4.2 Theory of Optimal Beamforming . . . . . . . . . . . . . 142

4.3 The FLAG Backend . . . . . . . . . . . . . . . . . . . 145

4.3 .1 General Overview . . . . . . . . . . . . . . . . . 145

4.4 The Data and Observations . . . . . . . . . . . . . . . . . 148

4.4 .1 Calibration Observations . . . . . . . . . . . . . . . . . . . 149

4.4 .2 Science Observations . . . . . . . . . . . . . . . . . . 152

4.4 .3 Data Reduction . . . . . . . . . . . . . . . . . . . . 153

4.4.3.1 Processing Beamforming Weights . . . . . . . . . 155

4.4 .4 Calibration and Imaging . . . . . . . . . . . . . . 156

1.5 Results . . . . . . . . . . . . . . . . . . . . 161

$1.5 .1 T_{\mathrm{sys}}$ and Noise Properties . . . . . . . . . . . . . . 161

1.5.2 Beamformer Weight Validity over Time . . . . . . . . . . 173

1.5.3 Hi FLAG Observations . . . . . . . . . . . . . . . . 176

1.5.3.1 NGC6946 . . . . . . . . . . . . . . . . 177

1.5.3.2 M31-M33 Cloud . . . . . . . . . . . . . . 189

1.6 Conclusions, Current Status and Future . . . . . . . . . . 197

5 Conclusions 201

5.1 Final Conclusions . . . . . . . . . . . . . . . . . . . 201

Appendix A Quantum Mechanical Derivation of the Spin-Flip Transition 205 


\section{List of Tables}

2.1 Summary of Observations and Properties . . . . . . . . . . . . . . 28

2.2 Summary of Data Cubes . . . . . . . . . . . . . . . . . . . . . . . . . . . . . . 77

2.3 Summary of Derived Properties . . . . . . . . . . . . . . . 79

3.1 Summary of Observations and Properties . . . . . . . . . . . 93

3.2 Q Parameters for the interactions between NGC4258, NGC4288, and UGC7408 . . . . . . . . . . . . . . . . . . . . . . . 100

3.3 Summary of Derived Properties . . . . . . . . . . . . . . . 132

4.1 Summary of FLAG Observations . . . . . . . . . . . . . . . 150

4.2 Formed Beam Offsets . . . . . . . . . . . . . . . . . . . 150

1.3 Mean $T_{\text {sys }} / \eta$ over all sessions . . . . . . . . . . . . . . . . . 165

1.4 Summary of derived system properties in XX Polarization from calibration scans used to make the science images . . . . . . . . . . . . . 167

1.5 Flux Offsets for NGC6946 Data . . . . . . . . . . . . . . . . . 187 


\section{List of Figures}

1.1 Star formation rate and cosmological density of Hi as a function of $z \quad 5$

2.1 GBT L-Band Beam Model . . . . . . . . . . . . . . . . . . . . . . . 33

2.2 NGC891 Moment Maps . . . . . . . . . . . . . . . . . . . . 45

2.3 NGC891 flux, cumulative Hi mass, and radial properties . . . . . . . 46

2.4 NGC891 Channel maps . . . . . . . . . . . . . . . . . . . . . . 48

2.5 NGC925 Moment Maps . . . . . . . . . . . . . . . . . . . . . 51

2.6 NGC925 flux, cumulative Hi mass, and radial properties . . . . . . . 53

2.7 NGC925 Channel Maps . . . . . . . . . . . . . . . . . . 55

2.8 Halogas flux . . . . . . . . . . . . . . . . . . . . 56

2.9 NGC4414 Moment Maps . . . . . . . . . . . . . . . . . . . . . . 58

2.10 NGC4414 flux, cumulative Hi mass, and radial properties . . . . . . . 59

2.11 NGC4414 Channel Maps . . . . . . . . . . . . . . . . . . . . . 60

2.12 NGC4565 Moment Maps . . . . . . . . . . . . . . . . . . 64

2.13 NGC4565 flux, cumulative Hi mass, and radial properties . . . . . . . 65

2.14 NGC4565 Channel Maps . . . . . . . . . . . . . . . . 66

$2.15 f_{19}$ of Pilot Survey Sources . . . . . . . . . . . . . . . 71

3.1 NGC949 Global Hi Profile . . . . . . . . . . . . . . . . . . . . . . . . 87

3.2 NGC4062 Global Hi Profile . . . . . . . . . . . . . . . . . . . . . . . 88

3.3 UGC2082 Global Hi Profile . . . . . . . . . . . . . . . . . . . . . . . 89

3.4 NGC4448 Global Hi Profile . . . . . . . . . . . . . . . . . . . . . . . 90

3.5 NGC4274 Global Hi Profile . . . . . . . . . . . . . . . . . . . . . . . 91

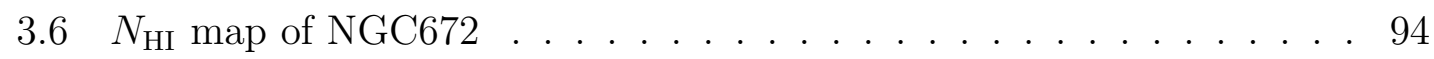

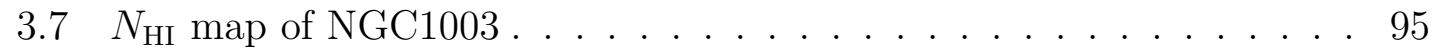

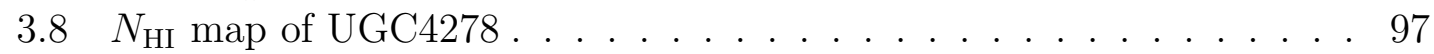

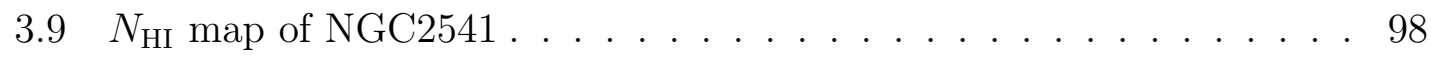

$3.10 N_{\mathrm{HI}}$ map of NGC4244 . . . . . . . . . . . . . . . . . . . . . . . . . . 99

$3.11 N_{\mathrm{HI}}$ map of NGC4258 field. . . . . . . . . . . . . . . . . 103

3.12 Channel maps of NGC4258 (367 $\mathrm{km} \mathrm{s}^{-1}$ to $\left.408 \mathrm{~km} \mathrm{~s}^{-1}\right)$. . . . . . . . 104

3.13 Channel maps of NGC4258 $\left(414 \mathrm{~km} \mathrm{~s}^{-1}\right.$ to $\left.455 \mathrm{~km} \mathrm{~s}^{-1}\right)$. . . . . . . 105

3.14 Zoomed $N_{\mathrm{HI}}$ image of NGC4258 Field . . . . . . . . . . . . . 106

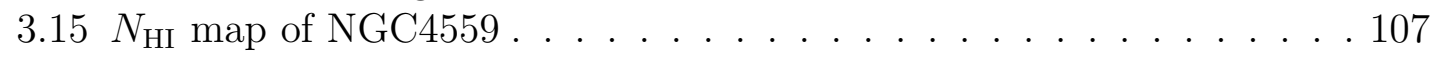

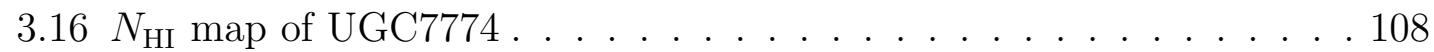

$3.17 N_{\mathrm{HI}}$ map of NGC4631 . . . . . . . . . . . . . . . . . . . 110

3.18 Channel Maps of NGC4631 (564 $\mathrm{km} \mathrm{s}^{-1}$ to $\left.605 \mathrm{~km} \mathrm{~s}-1\right)$. . . . . . 111

3.19 Channel Maps of NGC4631 (610 $\mathrm{km} \mathrm{s}^{-1}$ to $\left.651 \mathrm{~km} \mathrm{~s}-1\right)$. . . . . . . 112

3.20 Channel Maps of NGC4631 (656 $\mathrm{km} \mathrm{s}^{-1}$ to $\left.697 \mathrm{~km} \mathrm{~s}-1\right)$. . . . . . . 113

3.21 Channel Maps of NGC4631 $\left(703 \mathrm{~km} \mathrm{~s}^{-1}\right.$ to $\left.744 \mathrm{~km} \mathrm{~s}-1\right)$. . . . . . . 114

$3.22 N_{\mathrm{HI}}$ map of NGC5023 . . . . . . . . . . . . . . . . . . . . 115

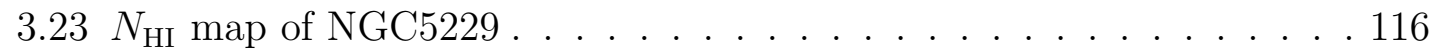

$3.24 N_{\mathrm{HI}}$ map of NGC5585 . . . . . . . . . . . . . . . . . . 117

3.25 NGC672 flux, cumulative Hi mass, and radial properties . . . . . . . 120 
3.26 NGC1003 flux, cumulative Hi mass, and radial properties . . . . . . . 121

3.27 UGC4278 flux, cumulative Hi mass, and radial properties . . . . . . . 122

3.28 NGC2541 flux, cumulative Hi mass, and radial properties . . . . . . . 123

3.29 NGC4244 flux, cumulative Hi mass, and radial properties . . . . . . . 124

3.30 NGC4258 flux, cumulative Hi mass, and radial properties . . . . . . . 125

3.31 NGC4559 flux, cumulative Hi mass, and radial properties . . . . . . . 126

3.32 UGC7774 flux, cumulative Hi mass, and radial properties . . . . . . . 127

3.33 NGC4631 flux, cumulative Hi mass, and radial properties . . . . . . . 128

3.34 NGC5023 flux, cumulative Hi mass, and radial properties . . . . . . . 129

3.35 NGC5229 flux, cumulative Hi mass, and radial properties . . . . . . . 130

3.36 NGC5585 flux, cumulative Hi mass, and radial properties . . . . . . . 131

$3.37 f_{19}$ vs. Galaxy Properties for Entire Survey Sample . . . . . . . . . . 135

4.1 Covariance Matrix Example . . . . . . . . . . . . . . . . . . 146

4.2 Calibraiton Grid Trajectory . . . . . . . . . . . . . . . . . . . . 149

4.3 Formay of Beamformer Weight Binary File . . . . . . . . . . . . . . . 154

1.4 Raw Beamformed Spectra of NGC6946 . . . . . . . . . . . . 159

$1.5 T_{\mathrm{sys}} / \eta$ as a Function of Frequency . . . . . . . . . . . . . . . 164

1.6 Temporal variation of $T_{\text {sys }} / \eta$ for the boresight beam . . . . . . . . . 166

1.7 Temporal variation of $T_{\mathrm{sys}} / \eta$ of Boresight Beam . . . . . . . . . . 168

1.8 Beamformer Weight Phase and Amplitude vs. Time . . . . . . . . . . 172

1.9 Hi flux profile of combined NGC6946 FLAG data . . . . . . . . . . . 178

1.10 Hi flux profile of NGC6946 FLAG data for GBT16B_400_12 . . . . . 179

1.11 Hi flux profile of NGC6946 FLAG data for GBT16B_400_13 . . . . . 180

1.12 Hi Profiles of Scaled GBT16B_400_12 Data . . . . . . . . . . . . . . . 181

1.13 Hi Profiles of Scaled GBT16B_400_13 Data . . . . . . . . . . . . . . . 182

1.14 Channel Maps of FLAG NGC6946 Combined Data . . . . . . . . . . 183

$1.15 N_{\mathrm{HI}}$ map of FLAG NGC6946 Data . . . . . . . . . . . . . . . . . . 184

1.16 Hi Profiles of Final Combined NGC6946 FLAG Data Cube . . . . . . 188

1.17 Individual Hi Flux Profiles of M31 Cloud 6 . . . . . . . . . . . . . . . 190

1.18 The Formed Beam Locations over the PAF FOV . . . . . . . . . . . . 191

1.19 The $T_{\mathrm{sys}} / \eta$ variations across the FOV . . . . . . . . . . . . . . . 192

1.20 Channel maps of the M31 Cloud 6 Snapshot image . . . . . . . . . 193

$1.21 N_{\mathrm{HI}}$ map of the M31 Cloud 6 Snapshot image . . . . . . . . . . . . . 194 


\section{Chapter 1}

\section{Introduction}

\subsection{The Utility of Neutral Hydrogen}

Because the proton and electron, which comprise the neutral hydrogen atom, are both Fermions (possessing half-integer spins, specifically 1/2), the interaction of their respective spin states in the presence of a magnetic field causes a spin-spin coupling that breaks the degeneracy of the ground state into two distinct triplet and singlet states (Kemble \& Zener, 1929). See Appendix A for a detailed quantum mechanical derivation. When the electron's spin is aligned with the proton, the total angular momentum is one and the atom is in an overall higher energy state than the anti-aligned state, where the total angular momentum is equal to zero. The difference between the potential energies of the two available states is equal to $\Delta E=9.427 \times 10^{-25} \mathrm{~J}$. Applying this energy difference

$$
\Delta E=h \nu
$$

where $h$ is the Planck constant, the frequency of the so-called spin-flip transition is found to be $\nu=1420.405751 \mathrm{MHz}$, or similarly a wavelength of $\lambda=21.10611405$ cm (Ewen \& Purcell, 1951).

This particular transmission frequency lies within the radio portion of the 
electromagnetic spectrum. Observations of the spectral line emission of neutral hydrogen (HI) with radio telescopes have provided insight to many diverse properties of galaxy structure and evolution. While considered a forbidden transition since the magnetic dipole approximation of the Hi atom has a rate of spontaneous emission of $\sim 3 \times 10^{15} \mathrm{~s}^{-1}$ (Mohr et al., 2016) — or taking the inverse, approximately $9.5 \times 10^{6}$ $\mathrm{yr}$ - the remarkably ubiquitous nature of $\mathrm{HI}$ in the Universe gives way to the detections of Hi over the entire sky in both our Galaxy as well as external galaxies. The low mass of an Hi atom makes it particularly susceptible to gravitational interactions, giving astronomers the capability to use HI as a detailed probe for galaxy kinematics, dynamical properties, morphology, and tidal interactions between galaxies (e.g., Eneev et al. 1973; Bosma 1983; Cox et al. 1996). Other examples of major scientific discoveries accredited to the analysis of Hi observational data are: tracing the distribution of dark matter in galaxies through the study of rotation curves (e.g., Roberts 1966), discerning the edges of late-type ${ }^{1}$ galaxies (e.g., van Gorkom 1996; Bland-Hawthorn et al. 2017; Ianjamasimanana et al. 2017), and the study of interstellar turbulence and the subsequent links to star formation in our own Galaxy, the Milky Way (MW; e.g., Pingel et al. 2013, 2018) and in external galaxies (e.g., Stanimirović et al. 1999; Zhang et al. 2012).

\subsection{Basic Observable Hi Properties}

As alluded to above, $\mathrm{HI}$ is a versatile tool for procuring a comprehensive understanding of both the MW and external galaxies. Since the transition frequency

\footnotetext{
${ }^{1}$ This description encompasses all classes of spiral and irregular galaxies.
} 
of $\mathrm{HI}$ is well constrained, one can utilize the principles of the Doppler effect to obtain a line-of-sight velocity that, in turn, can be used to model the kinematics of an observed Hi distribution. The intensity of the Hi emission observed by a radio telescope can be characterized by the brightness temperature, $T_{\mathrm{b}}$, which is a physical property of the radiation emitted by an object. It is formally defined as the temperature that a black body in thermal equilibrium must radiate at in order to replicate the observed radiation from a source. Its formal units are power per unit solid angle per unit bandwidth (in the Rayleigh-Jeans low frequency approximation for black body radiation). Depending upon the size of the radio telescope beam used for an observation, one can distinguish the intensity of the Hi distribution in two distinct ways. Firstly, if the solid angle subtended by the source $\left(\Omega_{\mathrm{s}}\right)$ is unresolved (smaller than the beam width, $\Omega_{\mathrm{b}}$ ), the measured brightness temperature will be equal to

$$
T_{\mathrm{b}}=T_{\mathrm{a}}\left(\frac{\Omega_{\mathrm{b}}}{\Omega_{\mathrm{s}}}\right)
$$

where $T_{\mathrm{a}}$ is the antenna temperature - the temperature at which a resistor would duplicate the output power per unit frequency (normalized by $k_{\mathrm{B}}$ ) of the entire system. Secondly, if the source emission is smooth and extends well beyond the size of the telescope beam, $T_{\mathrm{b}}$ becomes equivalent to the intrinsically measured $T_{\mathrm{a}}$, which can be taken as a direct measurement of the spectral brightness of the HI distribution.

Given a three dimensional distribution of Hi atoms in some space that has some density $n_{\mathrm{HI}}$, a quantity deemed the Hi column density $N_{\mathrm{HI}}$, which measures 
the amount of Hi atoms per square $\mathrm{cm}$ along the line-of-sight (los), can be calculated

$$
N_{\mathrm{HI}}=\int_{\operatorname{los}} n_{\mathrm{HI}} \mathrm{d} s
$$

where $\mathrm{d} s$ is the infinitesimal path length. Wilson et al. (2013) shows that if the standard assumption that the Hi in external galaxies is optically thin is applied, $N_{\mathrm{HI}}$ is then proportional to the velocity integrated brightness temperature

$$
N_{\mathrm{HI}}=1.82 \times 10^{18} \int_{l o s}\left(\frac{T_{\mathrm{b}}(v)}{\mathrm{K}}\right)\left(\frac{\mathrm{d} v}{\mathrm{~km} \mathrm{~s}^{-1}}\right) \mathrm{cm}^{-2},
$$

By obtaining many measurements over some area of sky that are sufficiently spaced such that they can be interpolated to create a grid, one can create a data cube with two spatial dimensions and one of frequency (or equivalently radial velocity with respect to some rest frequency) of an extended source. The fluctuations of the brightness temperature along the spectral axis of such cubes can be used to infer gas dynamics (e.g, by simultaneously plotting how brightness temperature changes as a function of position and radial velocity), or Equation 1.4 can be applied for each pixel in the two spatial dimensions to create integrated $N_{\mathrm{HI}}$ images that can probe the extended structure and morphology of the Hi in and around a given galaxy. Such cubes are the basic data sets for much of the analysis to be discussed in the subsequent chapters of this work. 


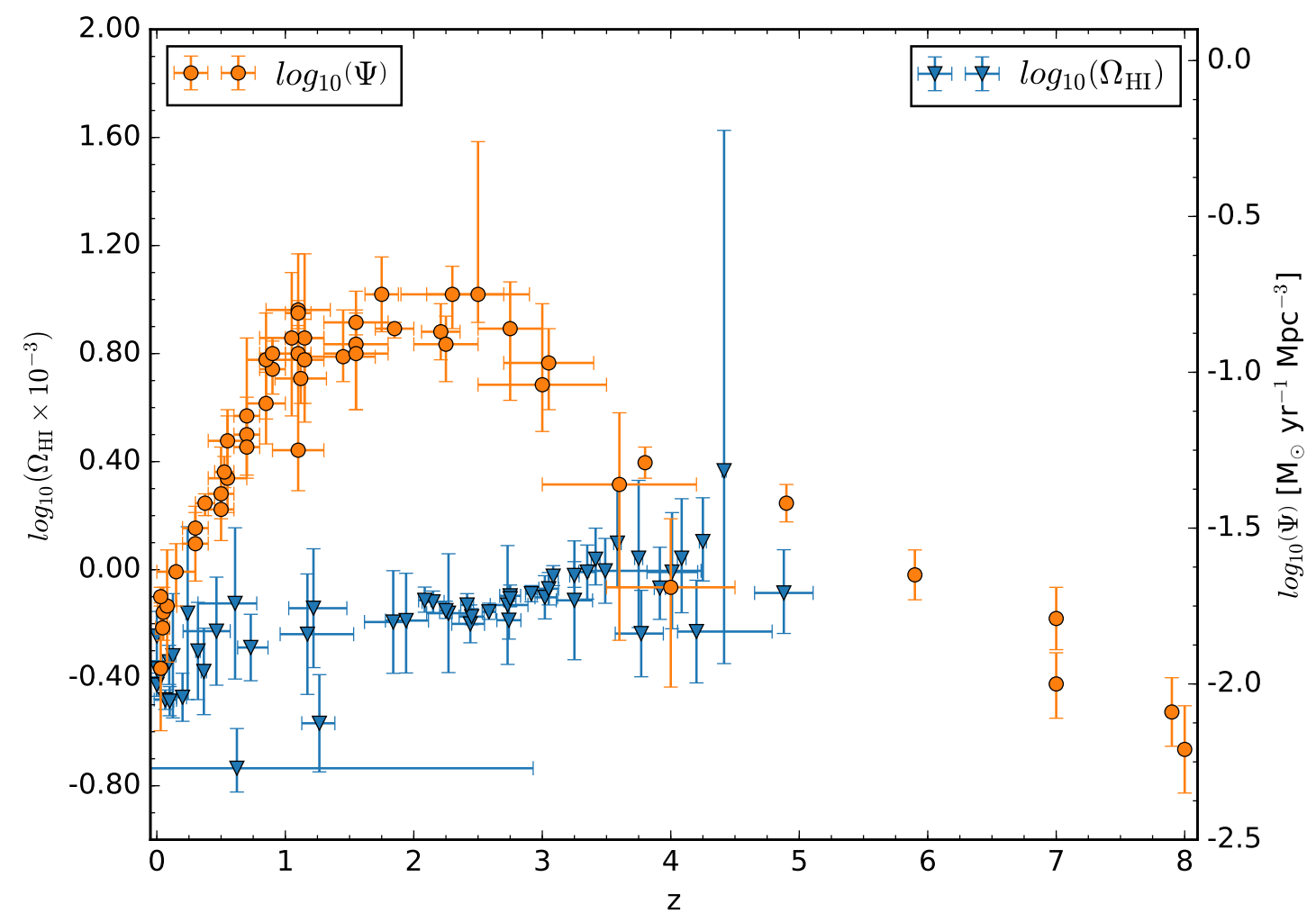

Figure 1.1 History of star formation rate from Madau \& Dickinson (2014) (right y-axis) plotted with the cosmological density of Hi (left y-axis) from Rhee et al. (2018) as a function of $z$ 


\subsection{Accretion of Hi Onto Galaxies}

The particular aspect of Hi this dissertation will focus on is the search for diffuse Hi in and around nearby, late-type galaxies. Throughout the text, 'diffuse'

refers to HI that is angularly extended with peak $N_{\mathrm{HI}}$ values below $10^{19} \mathrm{~cm}^{-2}$. This is the $N_{\mathrm{HI}}$ level is the sensitivity limits deep interferometric surveys. It is also near the $N_{\mathrm{HI}}$ threshold that models of extragalactic ionizing radiation predict ionization will be balanced with recombination.

There exists a discrepancy between the cosmological star formation rate density $(\Psi)$, which peaks near a redshift $(z)$ of $\sim 2$ (Madau \& Dickinson, 2014), and the relatively mild evolution of the cosmic neutral gas density $\left(\Omega_{\mathrm{HI}}\right)$ as traced by damped Lyman alpha (DLA) systems, nearby Hi emission, and spectral stacking of Hi data (Rhee et al., 2018). Figure 1.1 combines measurements of $\Psi$ compiled by Madau \& Dickinson (2014) with the $\Omega_{\mathrm{HI}}$ values published by Rhee et al. (2018) to explicitly demonstrate the deviation. As star formation rises by almost an order of magnitude over a $z$ range of 5 to 2 , or a look-back time between $12-10 \times 10^{6} \mathrm{yr}$, $\Omega_{\mathrm{HI}}$ shows little variation. Given that $\mathrm{HI}$ is a raw ingredient for the molecular hydrogen $\left(\mathrm{H}_{2}\right)$ gas required for the formation of stars (Prialnik, 2009), it is surprising to observe no obvious correlation between the distribution of neutral gas and star formation as a function of $z$. The implication of the inconsistent evolution of $\Omega_{\mathrm{HI}}$ with $\Psi$ is that a mechanism for refueling galaxies with the necessary cold gas to form stars remains undetected.

The theory of structure formation indicates that galaxies largely reside at the 
junction of large-scale filaments and sheets which compose the cosmic web (e.g., Frenk et al. 1985). Over-densities in the background baryonic (normal) matter and non-baryonic (dark matter) density distribution created gravitational instabilities that led to the formation of individual galaxies, groups and ultimately clusters. Analytic treatments of this process show that as baryons flow into the gravitational potential of spherical over-densities created by the dark matter, their temperature will increase such that the random motions outward will balance the gravitational pull inward. The system (i.e. the spherical over-density or 'halo') is then 'virialized' such that the kinetic energy $(K E)$ can be related to the potential energy $(U)$ like $K E=\frac{1}{2} U$

Imagine a system of $N$ individual stars that have the same average velocity and mass $m$ that are distributed throughout a sphere of radius $R_{\text {sys }}$. The total mass in the system is therefore $M_{\text {tot }}=N \cdot m$. The $K E$ of the entire system is then equal to

$$
K E=\frac{1}{2} M_{\mathrm{tot}} v^{2}
$$

while

$$
U=\frac{G M_{\mathrm{tot}}}{2 R_{\mathrm{sys}}},
$$

where $\mathrm{G}$ is the gravitational constant. Relating the average $K E$ to a temperature $T$ through the ideal gas law gives

$$
K E=3 / 2 k_{\mathrm{B}} T
$$


Here, $k_{\mathrm{B}}$ is the Boltzmann constant. For simplicity, if a spherically uniform density is assumed, $M_{\text {tot }}$ can be related to $R_{\text {sys }}$ and substituted into Equation 1.6. Assuming virial equilibrium where the particle motions balance out the tendency for collapse from the gravitational potential energy, one can set this form of Equation 1.6 equal to Equation 1.7 to get a scaling relation of $T_{\text {vir }} \propto M_{\text {vir }}^{2 / 3}$, where $T_{\text {vir }}$ is now the virial temperature and $M_{\text {vir }}$ is now the total mass contained within the halo. Given the mass of a typical dark matter halo $\left(\sim 10^{12} M_{\odot}\right)$, the temperature of the diffuse gas surrounding the new galaxy will be on the order of $10^{6} \mathrm{~K}$ ). In traditional views (e.g., Rees \& Ostriker 1977; White \& Frenk 1991), if the cooling time is short enough (either through recombination or collisional ionization/excitation processes), the virialized gas will eventual cool and fall onto the central galaxy to fuel its star formation. Recent numerical simulations such as Kereš et al. (2005, 2009) and Nelson et al. (2013), have shown that gas can accrete onto the central galaxy without heating up to the virial temperature. Since this gas has significantly shorter cooling times than the gas at the virial temperature, it will accrete onto the central galaxy essentially in free-fall in the form of vast, diffuse filaments which penetrate from near the edge of the halo down to the disk. The quasi-spherical accretion of hot gas with short cooling times has been deemed 'hot' mode accretion, while the accretion of gas through filaments of gas which never heated up the halo's virial temperature is called 'cold' mode accretion.

Figure 5 from Ocvirk et al. (2008) provides a visual demonstration of the spatial distribution (top two panels) and temperature (middle two panels) of the hot and cold gas. The left middle and top panels show the cool gas in the form of Mpc 
scale filaments that flow directly to the disk of the galaxy at high $z$. The right middle and top panels show that by lower $z$ the filaments have smoothed out to be a spherical distribution of uniformly high temperature gas. The temperature of the gas in these predicted cold filaments is too high within the streams themselves for a significant amount of neutral gas to exist within the largely ionized medium. However, as the gas approaches the disk, cooling instabilities in which gas is collisionally excited and cools through subsequent radiative de-excitation of excited states could allow gas to cool enough to form Hi in the innermost regions of the halo $(\mathrm{R} \leq 100 \mathrm{kpc})$ at $\left.N_{\mathrm{HI}} \leq 10^{18} \mathrm{~cm}^{-2}\right)$. Furthermore, extraplanar Hi gas has been observed in many nearby galaxies (e.g., Fraternali et al. 2002; Oosterloo et al. 2007; Kamphuis et al. 2013; Zschaechner et al. 2011), and while a majority of this gas is likely related to galactic fountain activity (Begeman, 1989), there is likely to be some associated with intergalactic gas (Sancisi et al., 2008). The Hi data presented in this work are deep enough to probe these levels in search of any hints of cold mode accretion and thus the missing star formation fuel. The possible existence of significant diffuse Hi components — both in terms of mass and spatial extent around nearby spiral galaxies is among the most captivating open questions in modern galaxy astrophysics. If detected, such a diffuse component could be associated with the infalling cold gas necessary for refueling future star formation.

While the detection of a considerable amount of diffuse Hi around multiple nearby galaxies would clarify how galaxies can continue with ongoing star formation, a non-detection has different but equally intriguing implications regarding our understanding of galaxy structure and evolution. One implication is that gas may come 
into galaxies in an ionized form. This may still even be true if the mass fraction of the potential diffuse $\mathrm{HI}$ is not high enough to account for the necessary accretion rates to fully refuel start formation. Another implication is a sensitivity threshold that future Hi surveys planned for the newest generations of radio telescopes, such as the Australian Square Kilometer Array Pathfinder and MeerKAT (and eventually the Square Kilometer Array), must meet to reliably detect diffuse HI. A non-detection at $N_{\mathrm{HI}} \sim 10^{18} \mathrm{~cm}^{-2}$ also has ramifications for the understanding of the physical conditions towards the edge of the disk of galaxies such as the transition from $\mathrm{HI}$ to ionized hydrogen (HII) and thus the definition of a distinct 'edge' of a galaxy. Models of the background extragalactic radiation field put forth by Maloney (1993) and Dove \& Shull (1994) predict the existence of a critical $N_{\mathrm{HI}}$ value on the order of $\sim 10^{18-17} \mathrm{~cm} \mathrm{~s}^{-1}$ wherein the Hi radial profile no longer smoothly decreases but declines sharply. If such a break were not present in deep Hi data, it would indicate that the ionizing flux of the extragalactic background radiation is not as influential at shaping the outer edge of galaxy disks. Furthermore, a smoothly decreasing HI profile would suggest the existence of an ultra-diffuse Hi component that may be associated with the large-scale structure of the cosmic web. Deep Hi observations performed with a telescope like the Green Bank Telescope (GBT), such as the those presented in this work, will reveal a comprehensive picture of the diffuse HI in and around spiral galaxies within the local Universe. 


\subsection{Spectral Line Observation with Phased Array Feeds}

The primary limiting factor in the study of diffuse Hi emission is the required integration time on large and generally oversubscribed radio telescopes. Furthermore, the field of view of conventional radio telescope at L-Band (frequency range of 1 to $2 \mathrm{GHz}$ ) is constrained to a single beam, primarily due to the large size of corrugated feed horns horns necessary at such wavelengths. While other large single dish facilities like Arecibo (e.g., Haynes \& ALFALFA Team 2008) and Parkes (e.g., Barnes et al. 2001) have successfully deployed multi-beam receivers, the engineering challenges of placing such large feeds (and associated cryogenics and electronics) in the focal plane are completely bypassed with a phased array feed (PAF). The limited field of view fundamentally restricts the required mapping time of extended sources. PAF receivers have the capability to form multiple beams on the sky thus increasing the field of view and, in turn, reducing the telescope time necessary to obtain deep data sets that probe the diffuse Hi in the local Universe.

While PAF technology has been prevalent in telecommunication applications for decades (Jeffs et al., 2008), the extremely low power levels of electromagnetic radiation from deep space has slowed development of PAF applications in radio astronomy. Only now, with the advancement of low noise amplifiers, cryogenics, and advanced signal processing algorithms, are astronomers able to utilize the advantages of PAFs. Several PAF systems are currently under development or beginning initial science surveys on various radio telescopes. The Australian Square Kilometer Array Pathfinder (ASKAP) telescope (DeBoer et al., 2009) possesses 36 dishes that, 
as a part of its early science and commissioning campaign, has imaged the nearby dwarf galaxy the Small Magellanic Cloud in unprecedented detail (McClure-Griffiths 2018; private communication); detected the distinct transient emission modes of an intermittent pulsar (Hobbs et al., 2016); demonstrated the wide-field imaging capabilities with the detections of over 3,000 distinct radio sources over a $6 \times 3$ square degree field of view (Heywood et al., 2016); and so far found over 20 Fast Radio Bursts (Bannister et al., 2017). The APERTIF upgrade to the Westerbork Radio Synthesis Telescope (Oosterloo et al., 2009) will bestow similarly increased capabilities to another prominent interferometer.

PAFs are also an invaluable upgrade to large single dish telescopes, which have the advantage over multi-antenna telescopes of continuous coverage in the visibility plane (that is, no spatial components of the incoming signals are filtered out) and phenomenal surface brightness sensitivity. Commissioning results on the Parkes radio telescope for an adapted PAF intended for use on the $100 \mathrm{~m}$ Effelsberg dish show good recovery of the Hi emission as detected on the existing multi-beam L-Band receiver (Reynolds et al., 2017).

In order to make PAFs a viable upgrade to reliable traditional single pixel feeds, the ratio of the system temperature $\left(T_{\mathrm{sys}}\right)$ to aperture efficiency $(\eta)$ of the PAF must be less than or equal to the value for the single pixel times the root number of formed beams. The system temperature is a measure of the available noise power introduced by the various elements of the receiver and its signal processing components, and $\eta$ describes how well the main reflector is illuminated. $T_{\text {sys }} / \eta$ ratio provides a way to measure the sensitivity of a radio telescope. 
Concerning a single dish like the GBT, a PAF operating between $\sim 1-2 \mathrm{GHz}$

must reach $T_{\text {sys }} / \eta<70 \mathrm{~K}$, assuming seven Nyquist spaced beams are formed. The Focal L-Band Array for the GBT (FLAG) is the worlds first cryogenically cooled PAF to be used on a large single dish. Subsequently, it is the most sensitive PAF in the world with $T_{\text {sys }}$ below $20 \mathrm{~K}$ at $1350 \mathrm{MHz}$ (Roshi et al., 2018). The excellent performance of this instrument makes it ideal for reaching previously unattainable $N_{H I}$ levels in a reasonable amount of telescope time. This work presents the results of deep Hi observations that test the feasibility of FLAG's role in the search for incredibly faint Hi that potentially is associated with accreting gas.

\subsection{Sampling the Focal Plane}

Consider a point source that illuminates the aperture of a parabolic radio telescope with diameter $D$ in the form of a monochromatic plane wave

$$
A(\mathbf{r}, t)=A_{0} e^{i(2 \pi \nu t+\phi)} e^{-i \hat{\mathbf{k}} \cdot \mathbf{r}},
$$

with an amplitude, $A_{0}$, frequency $\nu$, and phase $\phi$ that propagates in some direction $\hat{\mathbf{k}}$ over some time $t$. Here, r represents the three dimensional spatial coordinate.

The far-field approximation the of Kirchhoff's diffraction formula for a circular aperture gives the intensity pattern over some angle from the normal $\theta$ at the focal plane of the telescope as proportional to

$$
I(\theta) \propto\left(\frac{J_{1}(\pi D \sin (\theta) \nu / c)}{\pi D \sin (\theta)) \nu / c}\right)^{2}
$$


where $J_{1}$ is a Bessel function of the first kind and $c$ is the speed of light. The resulting pattern is what is known as an Airy pattern. The focal plane field will peak in the central region before gradually transforming to a series of concentric rings. This field will generate a complex voltage, $v$, in a sensor that sits within the focal plane. If multiple sensors are placed in the focal plane, the intensity pattern can be sampled at several different angle simultaneously to receive a different contribution from the incident energy. For example, a central sensor will inherently detect a larger intensity than an outer sensor when the source is lined up along the boresight of the telescope. That same outer sensor may also detect less intensity than a neighboring sensor if the companion sits within the one of the concentric rings of increased intensity. A beam can be formed by taking the weighted combination of the voltage received at each sensor (Fisher \& Bradley, 2000). Inherently, each sensor possesses a unique view of the aperture, which includes contribution from the ground. The ultimate goal of beamforming in the context of astronomical signal detection is to weight the measured voltage from each dipoles in such a way so as to reduce the amount of illumination spillover towards the ground, while maximizing gain by illuminating the aperture itself.

For a horizontal mount radio telescope such as the GBT, the direction of the propagation vector of an incident astronomical signal will depend on the Azimuth $(A z)$ and Elevation $(E l)$. Each plane wave from direction $(A z, E l)$ at frequency $\nu_{0}$ will correspond to an associated direction vector $\mathbf{a}\left(A z, E l, \nu_{0}\right)$. Solving for the direction vector over multiple sets of $(A z, E l)$ and summing the weighted response of individual sensors enables an observer to manipulate the general sensitivity of the 
array to particular areas of the sky.

\subsection{Dissertation Outline}

Chapter 2 presents the pilot survey of deep Hi observations down to a detection limit of $N_{\mathrm{HI}} \sim 10^{18} \mathrm{~cm}^{-2}$ performed with the GBT as part of the single dish counterpart to the WSRT Hydrogen Accretion in LOcal GAlaxieS (HALOGAS). The results for four sources out of a total of 24 nearby spiral galaxies are discussed in addition to a model of the GBT beam at L-Band. Chapter 3 extends this analysis to the remaining sources in the HALOGAS sample.

In Chapter 4, the results of several spectral line commissioning runs performed with the new beamforming backend for FLAG are presented. Discussions on the backend system, signal processing, custom software used in the data reduction and calibration procedure, and current issues to address going forward are included.

Lastly, Chapter 5 puts forth conclusions on the search for diffuse Hi emission, future role of PAFs in radio astronomy, and proposes additional avenues of analysis with these GBT HALOGAS data. Appendix A includes more in-depth discussions on the spin-flip transition of HI. 


\title{
Chapter 2
}

\section{A GBT Survey of the HALOGAS Galaxies and Their Environments}

\section{I: Revealing the full extent of Hi around NGC891, NGC925,}

\section{NGC4414 \& NGC4565}

\begin{abstract}
We present initial results from a deep neutral hydrogen (HI) survey of the HALOGAS galaxy sample, which includes the spiral galaxies NGC891, NGC925, NGC4414, and NGC4565, performed with the Robert C. Byrd Green Bank Telescope (GBT). The resulting observations cover at least four $\operatorname{deg}^{2}$ around these galaxies with an average $5 \sigma$ detection limit of $1.2 \times 10^{18}$ $\mathrm{cm}^{-2}$ over a velocity range of $20 \mathrm{~km} \mathrm{~s}^{-1}$ and angular scale of $9.1^{\prime}$. In addition to detecting the same total flux as the GBT data, the spatial distribution of the GBT and original Westerbork Synthesis Radio Telescope (WSRT) data match well at equal spatial resolutions. The Hi mass fraction below Hi column densities of $10^{19} \mathrm{~cm}^{-2}$ is, on average, $2 \%$. We discuss the possible origins of low column density Hi of nearby spiral galaxies. The absence of a considerable amount of newly detected Hi by the GBT indicates these galaxies do not have significant extended diffuse Hi structures, and suggests future surveys planned with the SKA and its precursors must go at least as deep as $10^{17}$
\end{abstract}

\footnotetext{
${ }^{1}$ This work is currently under revisions based on reviewer comments to be published in the Astrophysical Journal. I wish to acknowledge D. J. Pisano, G. Heald, T. H. Jarrett, W. J. G. de Blok, G. I. G. Józsa, E. Jütte, R. J. Rand, T. Oosterloo, and B. Winkel as coauthors on the submitted version of this chapter.
} 
$\mathrm{cm}^{-2}$ in column density to significantly increase the probability of detecting

Hi associated with the cosmic web and/or cold mode accretion.

\subsection{Introduction}

Resolved neutral hydrogen (HI) observations undertaken over the past decade have revealed many intricate details related to the morphology and dynamics of spiral galaxies. A primary science goal of recent large surveys is to develop a deep understanding of how physical processes within the disks of spiral galaxies, such as star formation and the subsequent stellar feedback, affect their local circumgalactic environments. Surveys such as The Hi Nearby Galaxy Survey (THINGS; Walter et al. 2008) and Hydrogen Accretion in LOcal GAlaxies Survey (HALOGAS; Heald et al. 2011; hereby referred to as H11) performed with the Very Large Array (VLA) and Westerbork Synthesis Radio Telescope (WSRT), respectively, provide high resolution maps of the environments around nearby spiral galaxies.

Accretion of diffuse gas onto the disks of galaxies from the intergalactic medium (IGM) is a possible explanation for how the Hi content of galaxies has remained relatively constant since $z \sim 2$ while the star formation rate was up to 10 times higher at high redshifts (Noterdaeme et al., 2012; Madau \& Dickinson, 2014). The constant Hi content implies that galaxies have somehow replenished themselves with enough gas to fuel continuous star formation. And though not directly responsible for star formation, Hi is an intermediate phase towards molecular hydrogen, which is the raw ingredient of the star formation fuel. If the star formation is to continue, 
external gas has to be accreted and pass through the Hi phase at some stage in the accretion process. Observationally inferred accretion rates as traced by Hi, however, fall between 0.1 and $0.2 M_{\odot}$ at low redshifts. This is a full order of magnitude lower than what is needed for galaxies to continually form stars at their current rates (Sancisi et al., 2008; Kauffmann et al., 2010). This discrepancy presents two intriguing scenarios: the cycle of star formation will eventually exhaust all of the available fuel within a few Gyr and star formation itself will gradually cease, or processes that refuel galaxies with the necessary gas have been missed by previous surveys. Numerical simulations have shown a likely mechanism for refueling star formation is through a quasi-spherical 'hot' mode and filamentary 'cold' mode (Kereš et al., 2005, 2009; Birnboim \& Dekel, 2003). Cold in the context of these numerical simulations refers to gas that has not been heated above the virial temperature of the galaxy's potential well $\left(\sim 10^{5} \mathrm{~K}\right)$, and hot refers to gas that has virialized in a process akin to the classical theory of galaxy formation in which shock-heated, virialized gas with short cooling timescales accretes onto the central galaxy (e.g., Rees \& Ostriker 1977). These simulations also suggest cold mode accretion was the dominant form of accretion at $z \geq 1$ for all systems, and remains prevalent through $z=0$ for galaxies in low-density environments $\left(n_{\text {gal }} \lesssim 1 \mathrm{~h}^{3} \mathrm{Mpc}^{-3}\right)$ and $M_{\text {halo }} \lesssim$ $10^{11.4} M_{\odot}\left(\right.$ or $\left.M_{\text {rmbary }} \leq 10^{10.3} M_{\odot}\right)$. For perspective, our own Milky Way has a virial (and thus halo) mass on the order of $10^{12} M_{\odot}$. These cold flows should exist in the form of vast filaments of cold, diffuse gas that permeate through the hot halo (Kereš et al., 2005). Comparisons by Nelson et al. (2013) between the smoothed particle hydrodynamic (SPH) numerical scheme employed in Kereš et al. (2005, 2009) and 
more sophisticated adaptive mesh refinement (AMR) simulations revealed the relative contribution of the cold mode is likely overestimated in earlier SPH simulations due to inherent numerical deficiencies. Nevertheless, the AMR simulations do show some fraction of gas is accreted cold.

The temperature of the gas in these predicted cold filaments is too high for a significant amount of neutral gas to exist within the largely ionized medium. However, AMR hydrodynamic simulations presented by Joung et al. (2012) show large amplitude non-linear perturbations can create cooling instabilities in which gas is collisionally excited and cools through subsequent radiative de-excitation of excited states. Large filamentary flows of inflowing gas are a possible seeding mechanism for non-linear perturbations, which allow gas to cool enough to form Hi clouds within the inner most regions of the halo $(\mathrm{R} \leq 100 \mathrm{kpc})$ at $\mathrm{HI}$ column densities $\left(N_{\mathrm{HI}} \leq\right.$ $10^{18} \mathrm{~cm}^{-2}$ ) currently detectable with existing telescopes.

More recent independent ballistic models show galactic fountain activity can account for the presence of extraplanar Hi around the Milky Way (in clouds like Complex C; Fraternali et al. 2015) and NGC891 (Fraternali \& Binney, 2006). In addition, Fraternali (2017) describes the condensation of hot coronal gas in the wake of the interaction with cooler galactic fountain gas, showing that fountain driven accretion can cool enough lower coronal gas to sufficiently extend the gas depletion time.

Observational evidence for predicted cold flows is very limited. Though absorption measurements, Stocke et al. (2010) and Ribaudo et al. (2011) both find low metallicity gas infalling onto a nearby solar metallicity Lymann Limit System 
whose mass is consistent with the presence of cold flows predicted by simulations. The presence of infalling, low metallicity gas is certainly consistent with cold flows, but these measurements do not reveal any information about the extended spatial distribution of the accretion. Absorption measurements are very promising in that they accurately probe the metallicity of galaxy halos, but such studies require a quasar or other bright background source to measure the absorption line of interest. Absorption studies of the Milky Way, in which these desired sightlines are abundant, show our own Galaxy is surrounded by an immense amount of low column density gas that is both ionized and neutral (e.g., Wakker et al. 2003; Richter et al. 2017) with temperatures ranging from $10^{2}$ to $10^{7} \mathrm{~K}$. Detection in emission does not rely on serendipitous sightlines required for external galaxies, and will constrain the large scale extent of the predicted cold flows or a potential diffuse component.

The unrivaled point source response of radio interferometers like the WSRT and VLA allows for incredible high resolution mapping capabilities at angular resolutions $\sim \frac{\lambda}{b_{\max }}$, where $b_{\max }$ is the maximum baseline $\left(b_{\max }=2.7 \mathrm{~km}\right.$ for the WSRT), which reveals the small scale structure of galaxies. On the downside, interferometers act as spatial filters by construction, and in particular due to the minimum possible spacing between neighboring telescopes in an interferometer (i.e., the physical size of each dish), there is a gap in $u-v$ coverage at large angular scales from the absence of short baselines. This gap is often referred to as the 'short-spacing' problem, and it limits the amount of large scale structure an interferometer is able to detect (e.g., Braun \& Walterbos 1985). As a consequence of the lack of sensitivity at large angular scales, past Hi observations performed with interferometers may 
have missed significant reservoirs of gas around galaxies. On the other hand, the HI is observed in channels covering small velocity ranges only, and thus potentially does not extend enough to cause the sampled baselines to miss several interesting low-density, diffuse features. The full $u-v$ coverage capability of single dish telescopes (Stanimirovic, 2002) permits the detection of structure at all angular scales to test that notion. The unblocked aperture design of the GBT and resulting low sidelobes coupled with the compromise between resolution $\left(9.1^{\prime}\right)$ and high surface brightness sensitivity $\left(\mathrm{T}_{\text {sys }} \lesssim 20 \mathrm{~K}\right)$ make it the ideal instrument to look for low column density structure around the HALOGAS sources.

The few surveys that have mapped down to $N_{\mathrm{HI}} \lesssim 10^{19} \mathrm{~cm}^{-2}$ have uncovered several interesting low density, diffuse features. Perhaps most notably, Braun \& Thilker (2004) discovered a low column density Hi filament connecting M31 and M33. Two possible explanations for its origin have been presented since its discovery: either it is similar to filaments seen in simulations of the cosmic web (Popping et al., 2009), and thus an observational example of the cold mode accretion process, or it was created via a past tidal interaction between M31 and M33 (Bekki, 2008; Putman et al., 2009). Higher resolution observations with the GBT by Wolfe et al. (2013) and Wolfe et al. (2016) show that this filament is clumpy in nature and made up of small Hi clouds with $\mathrm{M}_{\mathrm{HI}} \sim 10^{4-5} M_{\odot}, N_{\mathrm{HI}} \sim 10^{18} \mathrm{~cm}^{-2}$, and diameters on the order of kpc. M31 has a $\mathrm{M}_{\mathrm{dyn}} \sim 1.3 \times 10^{12} M_{\odot}$ (Corbelli et al., 2010), which suggests the cold mode accretion scenario is unlikely. Furthermore, the total Hi mass of these clouds is only $4.6 \times 10^{6} M_{\odot}$ providing only meager neutral mass accretion rates for a conservative infall time estimates of $10^{7-8}$ years. The origin of these 
clouds is still an open and intriguing question which can be answered by utilizing sensitive observations of the Hi within the circumgalactic environment of M31 and M33 (Wolfe et al., 2016).

Other recent detections by the GBT of large Hi structures in NGC6946 by Pisano (2014) and NGC2403 by de Blok et al. (2014a) suggest these features are seen around a variety of galaxies. In order to determine the true origin of these filaments, resolve the discrepancy between observed accretion rates and SFRs, and obtain a comprehensive understanding of how the disks of galaxies interact with their surrounding circumgalactic environment, a comprehensive Hi census spanning a wide range of astrophysically interesting properties (e.g., dynamical mass, total Hi mass, halo mass, SFRs, etc) is required. A complete census of these properties will build up large number statistics and uncover any underlying correlations between intrinsic galaxy properties and possible signatures of accretion. The HALOGAS observations of 24 nearby galaxies obtained with the WSRT and the THINGS survey with the VLA are critical steps towards just such a census. To ensure this census is absolutely complete, interferometer observations must be supplemented with large single dish observations to cover all angular scales to assure large-scale emission is not resolved out by interferometers, and to map down to the lowest possible column density levels.

In this chapter we present data and analysis from four sources from the HALOGAS survey: NGC891, NGC925, NGC4414, and NGC4565. These GBT maps are among the deepest $\left(N_{\mathrm{HI}} \sim 10^{18} \mathrm{~cm}^{-2}\right)$ for external galaxies obtained to date in HI. This chapter serves as an introduction to the full survey as a way to outline our anal- 
ysis methods and highlight our overall goals. In Section 2.2 we present an overview of the HALOGAS sample; the observing configuration, reduction strategy and a discussion on our GBT beam model and how we convolve the WSRT data to avoid contamination from extended structure are outlined in Section 2.3. The results from our comparison between the GBT and WSRT data for our initial four sources are discussed in Section 2.4 with an investigation into how the diffuse Hi environment relate to intrinsic galaxy properties following in Section 2.5. We then summarize our conclusions and commenting on future work in Section 2.6.

\subsection{The HALOGAS Data and Sample}

\subsubsection{WSRT Data Cubes}

The high-resolution HALOGAS cubes were produced from data obtained with the WSRT. See H11 for complete details on the observational configuration and data reduction of these data; see also Oosterloo et al. (2007) for configuration and reduction details specific to NGC891. A particular aspect of the HALOGAS observational setup we wish to highlight here is the minimum baseline length of $36 \mathrm{~m}$, which translates to a maximum recoverable angular scale of $\sim 20^{\prime}$. As mentioned in the Introduction, this particular angular scale is important because smooth emission extending above this limit will not be present in the WSRT data but fully observable by the GBT.

As will be discussed below, a significant portion of our analysis relies on convolved WSRT data convolved to the GBT resolution. Flux measurements from 
convolved data have the potential to be misleading as convolved emission will extend outside the original 'clean' boundaries used to produce the final interferometer cubes. Fully cleaned maps of array data are the sum of the restored clean components and residual map. Generally, only a portion of a map is cleaned, and thus, will have the correct flux. The flux in uncleaned portions of the map will be overestimated by a factor equal to the ratio of the dirty beam size to clean beam size. We measure outside of the clean region because the convolution obviously extends source emission beyond its original boundaries. Including regions of uncleaned emission in the convolution will inevitably lead to misleading total flux measurements as pixels with uncleaned emission have an intensity scale defined as Jy per dirty beam as opposed to Jy per clean beam (e.g., Jorsater \& van Moorsel 1995). In the interest of quantifying how the inclusion of unclean emission will affect the overall flux measurements in the convolved WSRT data, we extract two sub-maps from the original WSRT high-resolution NGC891 cubes that include a different number of pixels that were excluded from the cleaning as part of the data reduction. We then compare the total Hi flux values after convolving these sub-maps as described in Section 2.3.2. Specifically, $\sim 60 \%$ of the first extracted subregion includes pixels that were part of the original clean region, while the remaining $40 \%$ of the pixels in this sub-map were excluded from the cleaning algorithm. In the second sub-map, $90 \%$ of the pixels were cleaned during data reduction, while the remaining $10 \%$ of the pixels were excluded from cleaning. We find the total flux measurements of these two sub-maps to be the same. This indicates that while some uncleaned emission whether from pixels not inside the original clean region or simply low-level emission 
below the original clean threshold — is inevitably included, the total flux estimates are not significantly affected. That said, there are still systematic calibration uncertainties introduced by the specific treatment of the raw $u-v$ visibilities to consider. A few examples are the removal of residual baseline structure, flagged/missing baselines, antenna shadowing, and different weighting schemes applied to the complex visibilities. We adopt an overall systematic flux uncertainty of $5 \%$ to encapsulate uncertainties related to how the WSRT data were processed.

\subsubsection{Sample}

The total HALOGAS sample consists of 24 unbarred and barred, nearby spirals that span a very diverse range of astrophysically significant properties such as star formation rates $(\mathrm{SFRs})$, Hi mass $\left(\mathrm{M}_{\mathrm{HI}}\right)$, stellar mass $\left(\mathrm{M}_{*}\right)$, baryonic mass $\left(\mathrm{M}_{\text {bary }}\right)$, etc. The sample also consists of galaxies with a wide range of environments.

We adopt the best distance values listed for each source in H11. Given that the 'best' distances were determined by taking the median measurements from well established methods (e.g., Cepheid and/or tip of the red giant branch, planetarynebulae luminosity function, Tully-Fisher distances), we adopt a conservative 10\% overall uncertainty for these distances. The SFRs for all galaxies mentioned in this work besides NGC4565 and NGC2997 (to be discussed in Section 2.5.2 in regards to similar GBT data of nearby galaxies) are computed utilizing data from the $22 \mu \mathrm{m}$ band of the Wide-field Infrared Survey Explorer (WISE), a space based observatory deployed to map the entire sky in the infrared, along with far-ultraviolet (FUV) 
luminosity data from the Galaxy Evolution Explorer (GALEX; Gil de Paz et al. 2007). We follow the method outlined in Jarrett et al. (2013) to obtain an infrared SFR $\left(\mathrm{SFR}_{I R}\right)$ that traces star formation obscured by dust with further calibrations derived by Cluver et al. (2014) for the same IR bands. Due to the difficulty of disentangling the relative contribution from young and old stellar populations to various Polycyclic aromatic hydrocarbon emission bands near $12 \mu \mathrm{m}$, we chose to use the $\mathrm{SFR}_{I R}$ derived from the WISE $22 \mu \mathrm{m}$ band. The SFR tracing UV photons associated with young massive stars is given as

$$
\log _{10}\left(\frac{S F R_{F U V}}{M_{\odot} \mathrm{yr}^{-1}}\right)=\log _{10}\left(\frac{L_{F U V}}{L_{\odot}}\right)-9.69
$$

and is derived from the calibrations of Buat et al. (2008, 2011). Jarrett et al. (2013) combine $S F R_{\mathrm{IR}}$ and $S F R_{\mathrm{FUV}}$ to estimate a total SFR using the form

$$
S F R_{\mathrm{tot}}=\left(1-\eta_{0}\right) S F R_{\mathrm{IR}}+S F R_{\mathrm{FUV}}
$$

where $\eta_{0}$ represents the fractional contribution to the total IR emission from dust re-radiating energy injected from old stars; the value of $0.17 \pm 0.1$ is adopted (Buat et al., 2011; Jarrett et al., 2013). In the case of NGC4565, the SFR is taken from the HALOGAS calculations in Heald et al. (2012) since no FUV luminosities were available for these sources through $G A L E X$.

The $3.4 \mu \mathrm{m}$ band of WISE effectively traces light from old stars resulting in a practical measure of the stellar mass. Jarrett et al. (2013) show a linear trend exists 
between WISE W1-W2 and W2-W3 color and stellar mass-to-light ratios $(M / L)$. By relating the WISE color to 2 MASS $K_{\mathrm{s}}$ in-band luminosity, a $M / L$ ratio can be derived from the $K_{s}$ stellar mass relation of Zhu et al. (2010). This trend is further explored in Cluver et al. (2014), in which the W1-W2 color and stellar masses from the Galaxy And Mass Assembly (GAMA; Driver et al. 2009, 2011) survey are used for empirical calibration of the relationship. The best-fit for their sample including both passive and star-forming systems, but excluding known active galactic nuclei sources and WISE colors dominated by nuclear activity (W1-W2 $\geq 0.8$ ), is

$$
\log _{10}\left(\frac{M_{*} / M_{\odot}}{L_{\mathrm{W} 1} / L_{\odot}}\right)=-1.96\left(W_{3.4 \mu \mathrm{m}}-W_{4.6 \mu \mathrm{m}}\right)-0.03
$$

We adopt this relation to determine aggregate stellar masses for the HALOGAS sources. See Jarrett et al. (2013) and Cluver et al. (2014) for explicit details on the calculating of aggregate stellar masses utilizing WISE data. The adopted distance and SFRs from H11 are summarized in Table 2.1. See Table 2 in H11 for a complete summary of the targets' properties and explanation for how certain target properties such as SFRs and distances were derived. 


\begin{tabular}{|c|c|c|c|c|c|c|c|c|}
\hline Source & $\alpha^{\mathrm{a}}$ & $\delta^{\mathrm{b}}$ & Systemic Velocity $\left[\mathrm{km} \mathrm{s}^{-1}\right]$ & Total Bandwidth $[\mathrm{MHz}]$ & $\Delta v\left[\mathrm{~km} \mathrm{~s}^{-1}\right]^{\mathrm{c}}$ & $\sigma[\mathrm{mK}]^{\mathrm{d}}$ & $d_{\text {best }}[\mathrm{Mpc}]^{\mathrm{e}}$ & $\mathrm{SFR}_{t o t}\left[M_{\odot} \mathrm{yr}^{-1}\right]^{\mathrm{f}}$ \\
\hline NGC891 & $02^{h} 22^{m} 33.4^{s}$ & $42^{\circ} 20^{\prime} 57^{\prime \prime}$ & $528 \pm 4$ & 50.0 & 5.15 & 10 & $9.2 \pm 0.9$ & $3.92 \pm 1.75$ \\
\hline NGC925 & $02^{h} 27^{m} 16.9^{s}$ & $33^{\circ} 34^{\prime} 45^{\prime \prime}$ & $553 \pm 3$ & 50.0 & 5.15 & 12 & $9.1 \pm 0.9$ & $0.91 \pm 0.16$ \\
\hline NGC4414 & $12^{h} 26^{m} 27.1^{s}$ & $31^{\circ} 13^{\prime} 25^{\prime \prime}$ & $716 \pm 6$ & 23.4 & 5.15 & 13 & $18 \pm 2$ & $3.45 \pm 1.58$ \\
\hline NGC4565 & $12^{h} 36^{m} 20.8^{s}$ & $25^{\circ} 59^{\prime} 16^{\prime \prime}$ & $1230 \pm 5$ & 50.0 & 5.15 & 15 & $11 \pm 1$ & $0.67 \pm 0.10$ \\
\hline
\end{tabular}

Table 2.1 Summary of Observations and Properties

${ }^{a}$ Right Ascension (J2000)

${ }^{b}$ Declination (J2000)

${ }^{c}$ Velocity Resolution

${ }^{d}$ final rms noise per velocity channel

$e^{e}$ adopted distance from $\mathrm{H} 11$

${ }^{f}$ SFRs derived from WISE; value for NGC4565 taken from (Heald et al., 2012)

\subsection{GBT Observations and Data Reduction and Low-Resolution WSRT}

\section{Cubes}

\subsubsection{Observations and Data Reduction}

Our GBT maps were made in a "basket-weave" fashion by scanning the telescope for $2^{\circ} / 3^{\circ}$ along constant lines of Right Ascension $\left(\alpha_{\mathrm{J} 2000}\right)$ and Declination $\left(\delta_{\mathrm{J} 2000}\right)$ to stitch together a final $4 \operatorname{deg}^{2}\left(9 \operatorname{deg}^{2}\right.$ for NGC925) image (Mangum et al., 2007). If potential cold flows exist, their visibility in Hi depends on how close to the disk the relatively warm gas of the flow transitions to the Hi phase. For the range of distances of the sources presented in this work, the angular span of 2 deg corresponds to approximately $315 \mathrm{kpc}-620 \mathrm{kpc}$, which are sufficient to capture a majority of the virial volume. Each row or column is offset by $3^{\prime}$, and each scan consisted of a total of 72 separate $5 \mathrm{~s}$ integrations that were dumped every $100^{\prime \prime}$ to ensure Nyquist sampling. NGC891 and NGC925 were observed during January 2010 as part of the GBT project 10A-026, while NGC4565 and NGC4414 were ob- 
served during October 2013 and 2014 as part of GBT projects 13B-406 and 14B-293, respectively. We obtained an additional ten hours to map the inner $2 \mathrm{x} 1 \mathrm{deg}^{2}$ region of NGC891 as part of GBT project 16A-411. NGC925 and NGC4565 were observed with the GBT Spectrometer as the backend while the Versatile GBT Astronomical Spectrometer (VEGAS) was the backend used for the observations of NGC4414. The observations of NGC891 we present in this work combine the initial data from the GBT Spectrometer with the additional ten hours of data which utilized VEGAS as the backend. During each observation, the band was centered on the Hi line at the redshifts of the sources. The observing bandwidth, frequency resolution, noise at the native velocity resolution, and other data properties are summarized in Table 2.1. Calibration during the observation was done by frequency switching $-30.0 \%$ $(-23.4 \%$ for observations with VEGAS as the backend) of the total bandwidth from the center frequency at a one second period, and each observation session included time on $3 \mathrm{C} 48,3 \mathrm{C} 147$, or $3 \mathrm{C} 295$ as primary flux calibrator in order to compute a $\mathrm{T}_{\text {cal }}$ value for the noise diode. $\mathrm{T}_{\text {cal }}$ values computed for the Spectrometer varied between 1.53 and $1.57 \mathrm{~K}$ for both $\mathrm{XX}$ and $\mathrm{YY}$ polarizations with remarkably low scatter on the mean values of 1.56 and 1.57 for over a period of several months ( $1 \sigma$ $\sim 0.01 \mathrm{~K})$. The exceptional stability in the $\mathrm{T}_{\text {cal }}$ values translate to an upper limit on the uncertainty in the relative flux calibration to $<1 \%$ (more on the absolute calibration below). The computed $\mathrm{T}_{\text {cal }}$ values for observations performed with VEGAS as the backend showed a significant decrease in the stability. We compute the mean $\mathrm{T}_{\text {cal }}$ for VEGAS-only observations to be $1.41 \mathrm{~K}$ and $1.43 \mathrm{~K}$ for the $\mathrm{YY}$ and XX polarizations, respectively with a $1 \sigma$ uncertainty equal to $0.05 \mathrm{~K}$ for each. 
The increase scatter in the VEGAS $\mathrm{T}_{\text {cal }}$ was likely due to a crosstalk issue in the electronics of the backend that pushed the upper limit on the absolute flux calibration slightly upwards to $5 \%$, including other systematics such as baseline removal and, to a lesser extent, $\mathrm{T}_{\text {sys }}$ variation. The crosstalk issue manifested itself by offsetting the relative power levels between the XX and YY polarizations. This issue was fixed by the time we obtained our new data on NGC891 and consequently only NGC4414 data are affected. To ensure the derived $\mathrm{T}_{\text {cal }}$ values for VEGAS did not affect our flux calibration, we observed each HALOGAS source that used VEGAS as the backend in the Fall of 2016 as part of GBT project GBT16B-393. These observations were done explicitly in position-switching mode where the GBT was centered on source for five minutes, and then moved two degrees in Right Ascension to obtain a five minute off spectrum. Comparing spatially overlapping integrations between our original VEGAS data and those from the deep pointing observations showed very good agreement within the noise when the polarizations are averaged to derive the Stokes I component. We are therefore confident that the data presented here are not significantly affected by the large variation in the $\mathrm{T}_{\text {cal }}$ values. We still adopt an overall $5 \%$ flux uncertainty as an upper limit to account for possible errors in bandpass calibration, interference, and errors in modeling atmospheric effects. Given that the WSRT observations largely used the same calibrator sources, we are also confident in the relative flux calibrations between the two data sets. We determine the gain to be $1.86 \mathrm{~K} \mathrm{Jy}^{-1}$ based on the computed $\mathrm{T}_{\text {cal }}$ values and an $\sim 0.65$ aperture efficiency at $1420 \mathrm{MHz}$ (Boothroyd et al., 2011). The typical system temperature of observations fell between $15 \mathrm{~K}$ and $20 \mathrm{~K}$. 
The frequency switched data were reduced using a custom GBTIDL $^{1}$ routine to calculate a source temperature of the form

$$
T_{\mathrm{src}}=T_{\mathrm{Cal}} \cdot \frac{P_{\mathrm{s}}-\left\langle P_{\mathrm{s}, \mathrm{off}}\right\rangle}{\left\langle P_{\mathrm{s}, \mathrm{off}}^{\mathrm{CalOn}}-P_{\mathrm{s}, \mathrm{off}}^{\text {Caff }}\right\rangle},
$$

where the quantities in brackets denote averages of four integrations from each edge of the map for a total of eight 'off' integrations to increase the signal-tonoise. $P_{\mathrm{s}}$ refers to average power of the CalOn (noise diode on) and CalOff (noise diode off $)$ states - i.e., $(\mathrm{CalOn}+\mathrm{CalOff}) / 2$ - for a single integration in either the reference $(s=0)$ or signal $(s=1)$ bandpass switching state. $P_{\text {off }}$ is therefore the arithmetic mean of the CalOn and CalOff states for the 'off' spectrum generated from the average of the eight edge integrations in the corresponding bandpass state. Additionally, the denominator represents the average difference between CalOn and CalOff states for the corresponding switching bandpass. Equation 2.4 therefore calibrates our frequency-switched data as if we had position switched data. Using the edge of the map as a reference position is an advantage because we are able to obtain a reference spectrum for each RA/Dec scan without sacrificing telescope time to slew off source. In the case of NGC925, emission from a companion source near the edge of our map forced us to use eight contiguous integrations on the opposite map edge as our 'off' position. The reference bandpass $(s=0)$ is then shifted in frequency to match the signal bandpass $(s=1)$ and arithmetically averaged to improve the noise by a factor of $\sqrt{2}$. We then fit a third order polynomial to the

\footnotetext{
${ }^{1}$ http://gbtidl.nrao.edu/
} 
emission free regions of the spectra for all of our sources to remove baseline structure and any residual continuum sources leftover from our calibration procedure. In order to convert to units of $T_{\mathrm{A}}^{*}$ we assumed a constant zenith opacity appropriate for $21 \mathrm{~cm}$ observations of 0.01 (Chynoweth et al., 2011). We manually flagged ; $0.5 \%$ of all integrations due to broadband radio frequency interference using a custom graphical flagging GBTIDL routine. Finally, we used a boxcar smoothing function to produce raw GBT spectra at a velocity resolution of $5.15 \mathrm{~km} \mathrm{~s}^{-1}$. See Wolfe et al. (2016) and the GBT technical memo Wolfe et al. (2015) for a more comprehensive discussion on the mapping sensitivity, data acquisition, and reduction.

These calibrated data were then converted to a format readable by AIPS using the IDLTOSDFITS ${ }^{2}$ program and imported into AIPS where they were gridded into the final raw GBT data cubes using a convolution function of a Gaussian-tapered circular Bessel function (Mangum et al., 2007) within the task SDGRD.

\subsubsection{Convolution of WSRT data}

Accurate comparisons between high-resolution interferometer observations and single dish observations require the interferometer data to be smoothed to the resolution of the single-dish data set. Conventional analysis approximates the single-dish beam with a Gaussian smoothing function. While this is normally a good approximation, our goal of detecting low column density material around nearby galaxies requires a more comprehensive treatment since the Gaussian approximation for the

$\begin{array}{cll}{ }^{2} \text { Developed by Glen Langston of Nocumentation at } & \text { at } \\ \text { https://safe.nrao.edu/wiki/bin/view/GB/Data/IdlToSdfits. }\end{array}$




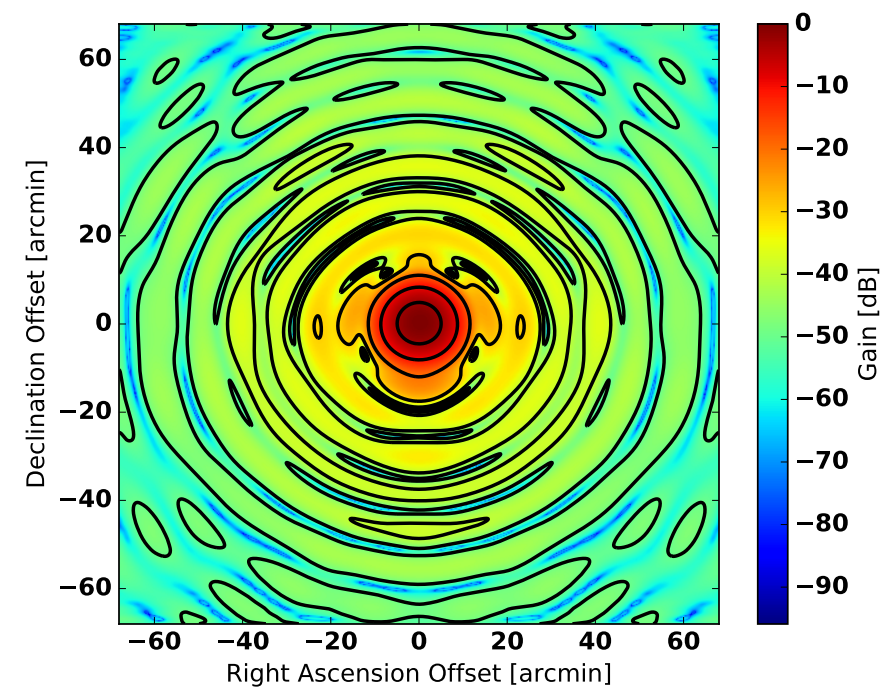

Figure 2.1 Normalized polar plot of a model GBT beam response on the sky. The contours represent dB levels of $-3,-10,-20,-30$, and -40 .

single-dish beam does not take into account radiation coming into the near sidelobes and wings of the main beam. Since our sources are well separated from Galactic Hi we are only concerned with emission originating from Hi in and near our target galaxies.

In order to account for extended emission coming into the GBT's innermost sidelobes, we construct a model beammap motivated from recent detailed simulations of the aperture illumination of the GBT at $1.4 \mathrm{GHz}$ (Srikanth 2017; private communication) as part of an ongoing project to produce a measured map of the GBT power pattern. We derive the model by taking the square of the forward Fourier Transform of a simulated in-focus aperture radiation pattern. An example of our calculated beam model is shown in Figure 2.1 as a contoured 2D polar plot. The farthest sidelobes in this map extend out approximately $1.2^{\circ}$ on the sky in radius between the -40 and $-50 \mathrm{~dB}$ level. Interestingly, the innermost sidelobes are not 
symmetric. Boothroyd et al. (2011) has thoroughly mapped the all-sky response of the GBT beam out at the farthest sidelobes. But due to system saturation effects within $1^{\circ}$ of the Sun, the nearest sidelobes, which are most susceptible to contamination from stray radiation in this work, were not able to be accurately mapped. The model presented in that paper (see their Figure 5) showed the sidelobes were highly symmetric. Emission entering the sidelobes at $\sim 1.2^{\circ}$ are not likely to contaminate our spectra as the gain here is approximately $50 \mathrm{~dB}$ down from the peak of the primary beam. However, the first sidelobe is only $30 \mathrm{~dB}$ down from the peak response. This makes contamination possible with some sources that have strong, extended emission out to $\sim 0.5^{\circ}$. We account for the possibility of contamination in the nearest sidelobes by using the GBT model beam map to include the sidelobe geometry in the convolution of the WSRT cubes. This provides the most accurate comparison possible between the two data sets without a genuine, measured beam map of the GBT.

There are, however, subtle consequences from utilizing a beam model with a set size as a smoothing kernel for high-resolution data. Namely, the final angular resolution of the convolved data will be slightly coarser than the GBT data because the high-resolution data are already convolved with the WSRT clean beam. Since the GBT beam model is not strictly Gaussian, we cannot solve for the optimal size of the kernel by taking the quadrature difference between the respective full-width half maximum (FWHM) values of the GBT and WSRT clean beam. We instead employ a Fourier Transform $(\mathfrak{F})$ deconvolution method wherein the $\mathfrak{F}$ of the GBT beam model $\left(\mathfrak{F}\left[\Omega_{\mathrm{GBT}}\right]\right)$ is divided by the $\mathfrak{F}$ of the WSRT clean beam $\left(\mathfrak{F}\left[\Omega_{\mathrm{WSRT}}\right]\right)$ to 
derive an optimal smoothing kernel, $K\{W S R T \rightarrow G B T\}$.

We follow a similar procedure described in Aniano et al. (2011) to apply the necessary tapers that reduce numerical noise introduced by the FFT algorithm. We first taper the FT of the GBT beam with a piecewise function of the form

$$
\Phi(k)= \begin{cases}1 & \text { if } k<k_{\alpha} \\ \exp \left[-\left(1.8249 \times\left(\frac{k-k_{\alpha}}{k_{\beta}-k_{\alpha}}\right)\right]\right. & \text { if } k_{\alpha} \leq k \leq k_{\beta} \\ 0 & \text { if } k_{\beta}<k\end{cases}
$$

where $k_{\beta}$ is taken to be the spatial frequency corresponding to four times the FWHM of the GBT and $k_{\alpha}=0.9 k_{\beta}$. We refer to the tapered form of $\mathfrak{F}\left[\Omega_{G B T}\right]$ as $\mathfrak{F}\left[\Omega_{G B T}\right]_{\Phi}$. The amount of power removed from tapering the high spatial frequencies is much less than $1 \%$ since almost all of the power is contained near spatial frequencies corresponding to the main beam. Nevertheless, it is important to ensure high spatial frequencies are near zero to avoid introducing numerical artifacts when $\mathfrak{F}\left[\Omega_{W S R T}\right]$ is divided out. We then take the reciprocal of $\mathfrak{F}\left[\Omega_{W S R T}\right]$ and taper the result by the low-pass filter

$$
f_{T}(k)= \begin{cases}1 & \text { if } k<k_{L} \\ 0.5 \times\left[1+\cos \left(\pi \times \frac{k-k_{\mathrm{L}}}{k_{\mathrm{H}}-k_{\mathrm{L}}}\right)\right] & \text { if } k_{\mathrm{L}} \leq k \leq k_{\mathrm{H}} \\ 0 & \text { if } k_{\mathrm{H}}<k .\end{cases}
$$

Here $k_{\mathrm{H}}$ is chosen such that $\mathfrak{F}\left[\Omega_{\mathrm{WSRT}}\right]\left(k_{\mathrm{H}}\right)=5 \times 10^{-3} \cdot \max \left(\mathfrak{F}\left[\Omega_{\mathrm{WSRT}}\right]\right)$ and $k_{\mathrm{L}}$ 
$=0.7 \times \mathrm{k}_{\mathrm{H}}$. We choose $k_{\mathrm{H}}$ as such to ensure a high spatial frequency cutoff where $\mathfrak{F}\left[\Omega_{\text {WSRT }}\right]$ is still appreciable, while the form of $k_{\mathrm{L}}$ is chosen to leave a majority of the lowest spatial frequency components unaffected by the filter.

The form of the optimal kernel is therefore given as

$$
K\{W S R T \rightarrow G B T\}=\left|\mathfrak{F}^{-1}\left[\mathfrak{F}\left[\Omega_{\mathrm{GBT}}\right]_{\Phi} \times \frac{f_{\mathrm{T}}}{\mathfrak{F}\left[\Omega_{\mathrm{WSRT}}\right]}\right]\right|
$$

where $\left|\mathfrak{F}^{-1}\right|$ represents the magnitude of the inverse Fourier Transform back to the sky plane. We note that Aniano et al. (2011) worked strictly with the real components since their kernels were largely rotationally symmetric. Since the WSRT clean beams are generally not symmetric, their Fourier Transforms will not be rotationally symmetric. As such, we work with the polar forms of $\mathfrak{F}\left[\Omega_{\mathrm{WSRT}}\right]$ and $\mathfrak{F}\left[\Omega_{\mathrm{GBT}}\right]$ to preserve the phase contribution. The resulting smoothing kernels are, as expected, marginally narrower than the GBT beam model with the largest residuals $(\leq 0.01 \%)$ occurring towards the center.

\subsubsection{Primary-beam Correction}

The standard reduction techniques of imaging and deconvolution of interferometer data result in a model representation of the sky multiplied by the primary-beam response of the antennas. The most accurate measure of flux requires a 'primarybeam correction' to the final data products, which we define as dividing out the primary-beam response of each velocity plane in the cube. We remove the primarybeam response from the native high-resolution WSRT cubes in the Miriad software 


\begin{tabular}{lcccccc}
\hline \hline Source & $\theta_{\text {maj }}[\operatorname{arcseconds}]$ & $\theta_{\text {min }}[\operatorname{arcseconds}]$ & $\sigma[\mathrm{K}]$ & $\mathrm{S}\left[\mathrm{Jy} \mathrm{km} \mathrm{s}{ }^{-1}\right]$ & $3 \sigma N_{\mathrm{HI}}\left[\mathrm{cm}^{-2}\right]$ & $\Delta \mathrm{v}\left[\mathrm{km} \mathrm{s}^{-1}\right]$ \\
\hline NGC891 & & & & & & \\
High-Res WSRT & 28.0 & 21.4 & 0.100 & $(1.90 \pm 0.09) \times 10^{2}$ & $6.7 \times 10^{19}$ & 8.24 \\
Conv. WSRT & 546.0 & 546.0 & 0.003 & $(1.91 \pm 0.09) \times 10^{2}$ & $2.0 \times 10^{18}$ & 8.24 \\
Regridded GBT & 546.0 & 546.0 & 0.010 & $(1.93 \pm 0.09) \times 10^{2}$ & $6.8 \times 10^{18}$ & 8.24 \\
\hline NGC925 & & & & & 4.12 \\
High-Res WSRT & 37.9 & 33.2 & 0.130 & $(2.85 \pm 0.14) \times 10^{2}$ & $2.4 \times 10^{19}$ & 4.12 \\
Conv. WSRT & 546.0 & 546.0 & 0.003 & $(2.84 \pm 0.14) \times 10^{2}$ & $1.8 \times 10^{18}$ & 4.12 \\
Regridded GBT & 546.0 & 546.0 & 0.013 & $(2.96 \pm 0.15) \times 10^{2}$ & $4.0 \times 10^{18}$ & 4.12 \\
\hline NGC4414 & & & & & \\
High-Res WSRT & 39.0 & 33.5 & 0.130 & $(6.2 \pm 0.3) \times 10^{1}$ & $5.2 \times 10^{19}$ & 4.12 \\
Conv. WSRT & 546.0 & 546.0 & 0.005 & $(6.1 \pm 0.3) \times 10^{1}$ & $2.0 \times 10^{18}$ & \\
Regridded GBT & 546.0 & 546.0 & 0.013 & $(7.3 \pm 0.4) \times 10^{1}$ & $5.2 \times 10^{18}$ & 4.12 \\
\hline NGC4565 & & & & & 4.12 \\
High-Res WSRT & 33.5 & 30.8 & 0.150 & $(2.74 \pm 0.14) \times 10^{2}$ & $4.8 \times 10^{19}$ & 4.12 \\
Conv. WSRT & 546.0 & 546.0 & 0.004 & $(2.71 \pm 0.14) \times 10^{2}$ & $1.7 \times 10^{18}$ & \\
Regridded GBT & 546.0 & 546.0 & 0.015 & $(2.66 \pm 0.13) \times 10^{2}$ & $7.0 \times 10^{18}$ & \\
\hline
\end{tabular}

Table 2.2 Summary of Data Cubes

package (Sault et al., 1995) with the same beam model used in H11. The primarybeam correction is applied before convolution as this most accurately represents the sky distribution observed by the interferometer. Furthermore, since the resulting low-resolution data cube must be scaled by the ratio of the GBT beam to the smaller WSRT synthesized beam to account for resolution differences when measuring the total flux, we found that removing the primary beam response after convolution does not conserve the total flux value computed for the high-resolution cube. To avoid issues with the non-uniform noise properties towards the edge, we extract a sub-cube such that all spatial scales fall within the half-power point of the WSRT primary beam for our subsequent analysis. 


\subsection{Results}

\subsubsection{Summary of Analysis}

In the following section, we present initial analysis for four (NGC891, NGC925, NGC4414, and NGC4565) of the 24 total sources in the HALOGAS sample, By analyzing $\mathrm{HI}$ at various angular resolution and sensitivities, the extent of Hi environ-

ment of these galaxies between $18 \lesssim \log _{10}\left(N_{\mathrm{HI}} / \mathrm{cm}^{2}\right) \lesssim 21$ can be fully characterized. Before discussing results for individual sources, we first summarize the steps of our analysis.

\subsubsection{Global Hi Profiles and Noise}

The flux as a function of velocity measured in the three data cubes is shown in each target's respective subsection. We first use the Miriad task REGRID to regrid each GBT cube to be on the same angular and spectral scale as their WSRT counterpart. We estimate the noise properties within each respective cube, $\sigma(x, y)$, by fitting the negative half of a histogram whose pixels values were drawn from a region with no emission in all velocity channels (i.e., those with and without emission) with a Gaussian. We determine the $1 \sigma$ noise in the native, primarybeam corrected high-resolution WSRT data cubes to be between 100 and $150 \mathrm{mK}$, between $10 \mathrm{mK}$ and $15 \mathrm{mK}$ for the regridded GBT cubes, and between 5 and $10 \mathrm{mK}$ for the convolved, primary-beam corrected WSRT cubes. These noise properties are summarized in the fourth column of Table 2.2 


\subsubsection{2 $N_{\mathrm{HI}}$ Images}

Since we are mostly interested in the low column density environments of our sources, care must be taken to correctly scale the convolved data, distinguish signal from noise, as well as calculating associated uncertainties. We do this for the cumulative Hi mass as a function of radius and $N_{\mathrm{HI}}$.

To this end, we determine the gain to convert the convolved, primary-beam corrected WSRT cubes from Jy/Beam to brightness temperature in units of Kelvin using the equation

$$
T_{\mathrm{b}}=\frac{\lambda^{2} S}{2 k \Omega_{\mathrm{a}}}
$$

Here $S$ is the flux density, $\Omega_{\mathrm{a}}$ is the beam area, $k$ is the Boltzmann constant and $\lambda$ is the wavelength of the observation (i.e., 0.211 meters). Taking these values and simplifying we arrive at

$$
T_{\mathrm{b}}[\mathrm{K}]=\frac{6.87 \times 10^{5} S[\mathrm{Jy} / \mathrm{beam}]}{\Omega_{\mathrm{a}}\left[\operatorname{arcseconds}^{2}\right]}
$$

The area of the GBT beam model used in this study is $3.69 \times 10^{5}$ square arcseconds, while the area of the WSRT clean beam can be approximated as a Gaussian and is given by $1.1331 \times\left(\theta_{\mathrm{maj}} \cdot \theta_{\mathrm{min}}\right)$ (the major and minor axis in arcseconds, respectively). If one multiplies Equation 2.7 by the reciprocal of of the flux density, $S$, and plugs in the area of the GBT beam model, it returns the gain factor of $1.86[\mathrm{~K} / \mathrm{Jy}]$ as derived in Section 2.3.1.

Computing the noise on an individual pixel basis is imperative to the treat- 
ment of the WSRT data (at both high and low resolutions) since the primary-beam correction changes the behavior of the noise as a function of position. The noise in the GBT data is relatively uniform over the cube, though the characterization of individual pixel noise is useful for constraining uncertainties in subsequent analysis. Since the original WSRT cubes were Hanning smoothed to their final velocity resolution, the pixels along the velocity axis are not independent. Verheijen \& Sancisi (2001) show an associated $1 \sigma$ noise map can be computed by

$$
\sigma_{\mathrm{N}}(x, y)=\sqrt{\left(N(x, y)-\frac{3}{4}\right)} \frac{4}{\sqrt{6}} \sigma(x, y),
$$

where $N(x, y)$ is the number of pixels used in the integration. For each data cube we produce masked (where emission below some threshold is blanked) and unmasked $N_{\mathrm{HI}}$ images. Masked images are useful when studying the radial dependence on column density since we wish to characterize the spatial variations of low-level signal, while unmasked images are used when studying the properties of the total flux.

We follow Verheijen \& Sancisi (2001) and Lelli et al. (2014) by first constructing a mask for our high-resolution data by spatially smoothing the high-resolution cubes to $40^{\prime \prime}\left(50^{\prime \prime}\right.$ in the case of NGC4414) and only include pixels above $3 \sigma(x, y)$ — as determined from a fit to the negative half of a histogram — in the sum. As a consequence of this mask application, the number of channels used in the sum, and thus the uncertainty, will vary pixel-to-pixel in the resulting $1 \sigma$ noise maps. Due to the variation across the map, a global $3 \sigma$-level is no longer straightforward to calculate. We calculate a global $3 \sigma N_{\mathrm{HI}^{-}}$level in these cubes by creating a signal-to-noise 
$(\mathrm{S} / \mathrm{N})$ map by taking the ratio of the masked $N_{\mathrm{HI}}$ images with the $1 \sigma$ noise maps.

We then take the average of pixels in the $\mathrm{S} / \mathrm{N}$ maps satisfying $2.75 \leq \mathrm{S} / \mathrm{N} \leq 3.25$ to ensure a large enough sample to compute a reliable mean value. The final $N_{\mathrm{HI}}$ images only contain pixels whose value lies above this $3 \sigma$-level. In the case of the low-resolution WSRT and GBT data, we repeat the masking procedure as described above but directly discard pixels which do not meet the $3 \sigma(x, y)$ threshold without constructing a spatially coarser cube. The beam sizes, $\sigma(x, y)$ values, $\sigma(x, y)$, total flux, the $N_{\mathrm{HI}} 3 \sigma$-level, and velocity resolutions for the data sets are summarized in Table 2.2.

\subsubsection{The Cumulative Hi Mass vs. $N_{\mathrm{HI}}$}

Since HI mass is simply proportional to column density times a physical area, and we have the distance to each source, we can convert an individual pixel value of column density to an equivalent Hi mass to determine the Hi mass probability distribution function, which measures the total HI contained within discrete $N_{\mathrm{HI}}$ bins. Integrating this distribution therefore gives the cumulative Hi mass as function of $N_{\mathrm{HI}}$, which measures the total HI mass for pixels equal to or exceeding $N_{\mathrm{HI}}$ bins. This distribution conveniently describes the fraction of Hi mass above and below distinct $N_{\mathrm{HI}}$ thresholds. We can use the cumulative Hi mass distribution as a diagnostic for how well the WSRT data recover the extended HI around these sources. For example, if the GBT data detected an extended diffuse Hi feature that was resolved out by the WSRT, the cumulative Hi mass distribution should deviate 
at lower column densities. In all cases presented here, the profiles flatten out well before the $N_{\mathrm{HI}} 3 \sigma$-level listed in Table 2.2. We set the lowest bin to be equal to one half the listed $N_{\mathrm{HI}}$ value in order to avoid the inclusion of noisy pixels and focus on the behavior between low and intermediate $N_{\mathrm{HI}}$ levels. The maximum bin size is set to $90 \%$ of the peak $N_{\mathrm{HI}}$ value. Note that the cumulative Hi mass as a function of $N_{\mathrm{HI}}$ from both telescopes are normalized by the total GBT Hi mass.

One aspect of concern when analyzing low-resolution data is whether emission adequately fills the larger GBT beam. In order to simulate the effect of this analysis on an unresolved source — where the low resolution map would simply trace the response of the GBT beam — we scale the GBT beam model introduced in Section 2.3.2 to the peak column density value of the GBT map and repeat the analysis calculating the cumulative HI mass as a function of $N_{\mathrm{HI}}$ (we do the same for the radial profiles of the of $N_{\mathrm{HI}}$ discussed in the next subsection). While the resolution effects will be source dependent, it is generally true that the large GBT beam does not hinder this (nor the radial average of $N_{\mathrm{HI}}$ ) analysis until the highest $N_{\mathrm{HI}}$ bins, which trace the structure of the main lobe.

\subsubsection{Radial Profiles of $N_{\mathrm{HI}}$ and Cumulative Flux}

The radial functions of the mean column density and cumulative flux are useful to compare how the properties of the HI emission detected by the GBT and WSRT change at various angular extents. For example, a large positive offset of cumulative flux and mean $N_{\mathrm{HI}}$ at large radii in the GBT data would indicate the WSRT resolved 
out large-scale Hi emission. Additionally, profiles of the cumulative flux as a function of radius that do not flatten out or begin to dip at large radii may trace artifacts (e.g., negative bowls) in the high-resolution WSRT data. In the analysis of the radial extent of low column density structure, we use the masked $N_{\mathrm{HI}}$ images to ensure low-level signal has not been buried in the noise. The cumulative flux as a function of radius is derived from unmasked maps to best probe the radial variations in total flux. We also note that by measuring properties contained within radial bins overlaid on non-axisymmetric structure, the deviations at large radii inherently only reflect the distribution of Hi along the major axis; thus, these plots do not reveal where potential differences between the maps occur. Nevertheless, any potential excess HI emission originating from structures spanning larger angular scales than what the WSRT is sensitive to should be evident even in radially averaged quantities. Finally, we note that no correction for inclination angle has been applied in the computation of these radial profiles. While the inclination of our sources range between $\sim 50 \mathrm{deg}$ to $\sim 90 \mathrm{deg}$, we are most interested in the relative difference between the profiles derived between the GBT and WSRT data sets rather than how the radial properties change source-to-source. This relative offset between data sets for a particular source will be the unaffected by the correction factor of the cosine of the inclination angle.

As a further check of resolution effects, the solid black line in the radial column density profiles again denote the GBT beam model scaled to the peak $N_{\mathrm{HI}}$ value of the GBT data. Again, the model of an unresolved source deviates sufficiently well from the data indicating this analysis is not particularly hindered by resolution effects. 


\subsubsection{NGC891}

NGC891 is an edge on $\left(i \gtrsim 89^{\circ}\right) \mathrm{Sb} / \mathrm{SBb}$ galaxy whose Hi has been extensively studied over the past two decades (e.g., Sancisi \& Allen 1979; Rupen 1991; Swaters et al. 1997; Oosterloo et al. 2007). Utilizing the WSRT, Oosterloo et al. (2007) made deep, high-resolution maps of NGC891. These deep maps revealed a huge galactic halo containing $30 \%$ of the total Hi mass. Other significant results from this study were: the discovery of a filament extending $22 \mathrm{kpc}$ in projection from the disk towards the companion, UGC1807; counter-rotating Hi clouds in the halo; and differential rotation lagging with respect to the disk. Models of interactions between material from galactic fountain activity and hot coronal gas have successfully recreated the observed velocity rotational velocity gradient (Marinacci et al., 2010; Fraternali, 2017). Halo pressure gradients and magnetic tension likely contribute as well (Benjamin, 2002). See Figure 2.2 for the masked integrated Hi image and velocity fields of NGC891 at low and high resolution. The GBT data do not reveal any low level emission extending from NGC891 to its companion, UGC1807.

The global flux density profiles for NGC891 derived from the GBT and WSRT data are shown in Figure 2.3a. There is excellent consistency between the profiles. The total measured flux for the GBT data is $193 \pm 9 \mathrm{Jy} \mathrm{km} \mathrm{s}{ }^{-1}$. The flux density measured by the GBT translates to a total Hi mass of $(3.86 \pm 0.19) \times 10^{9} M_{\odot}$ at the adopted distance of $9.2 \pm 0.9 \mathrm{Mpc}$ from H11. The flux values for the convolved and high-resolution WSRT data are measured respectively to be $191 \pm 9 \mathrm{Jy} \mathrm{km} \mathrm{s}^{-1}$ and $190 \pm 9 \mathrm{Jy} \mathrm{km} \mathrm{s}^{-1}$. 

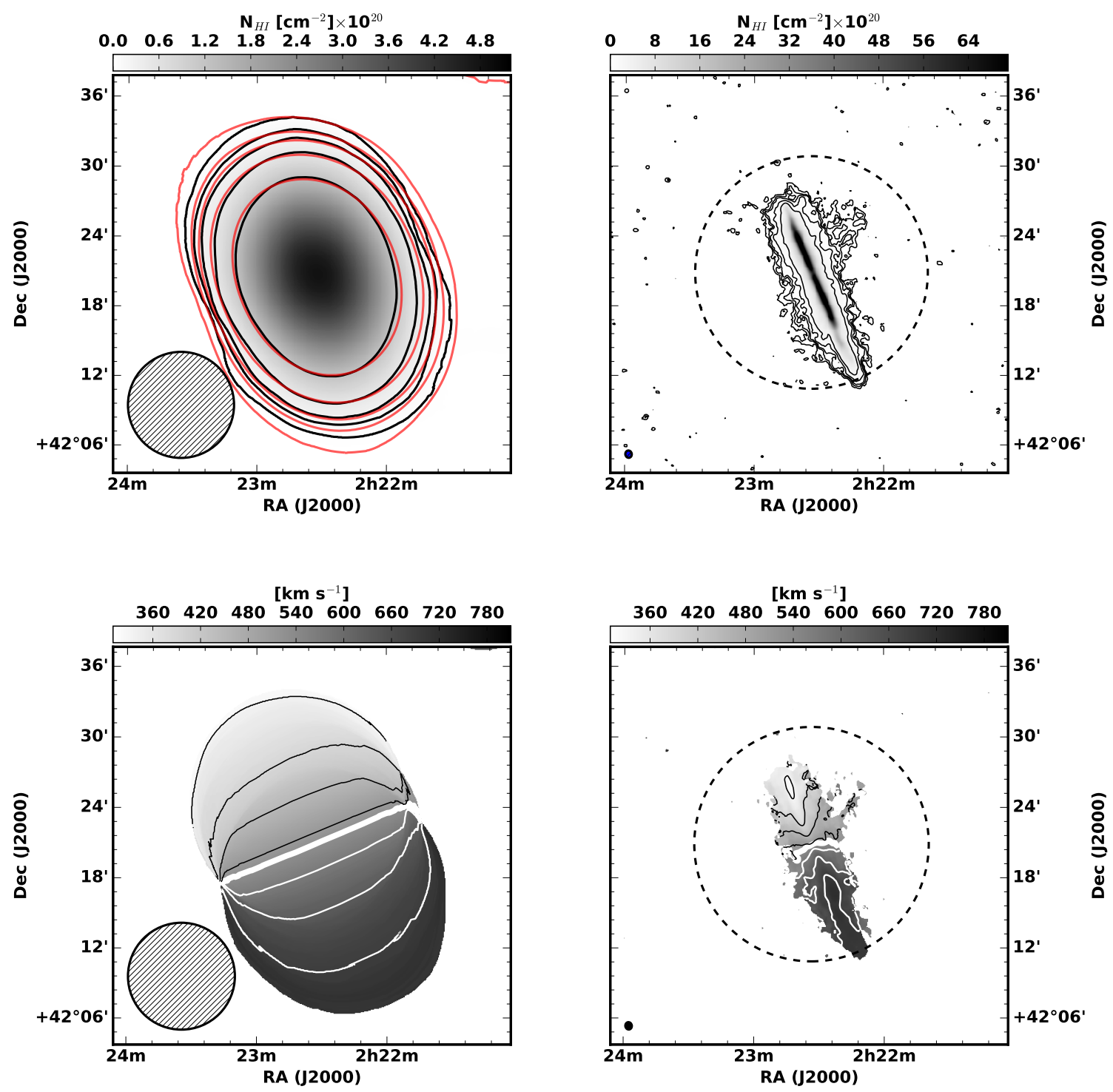

Figure 2.2 Integrated Hi image (top) and velocity fields (bottom) for the lowresolution (left) and high-resolution WSRT (right) data for NGC891. The contours in the low-resolution $N_{\mathrm{HI}}$ images start at a column density value of $5 \times 10^{18} \mathrm{~cm}^{-2}$ and continue at 3, 5, 10, and 25 times that level. The black and red contours respectively denote the regridded GBT and convolved WSRT data. The contours in the associated high-resolution image begin at a level equivalent to $2 \times 10^{19} \mathrm{~cm}^{-2}$ and continue at $3,5,10$, and 25 times that level. Note that the low-resolution velocity field is derived only from the GBT data cube. The contours in both velocity fields begin at $330 \mathrm{~km} \mathrm{~s}^{-1}$ and continue in steps of $60 \mathrm{~km} \mathrm{~s}^{-1}$. The systemic velocity of $528 \mathrm{~km}$ $\mathrm{s}^{-1}$ is represented by the thick line, and the approaching and receding velocities are denoted by black and white contours, respectively. The dashed circles in the right two panels represent the maximum recoverable angular scale of the WSRT data. 

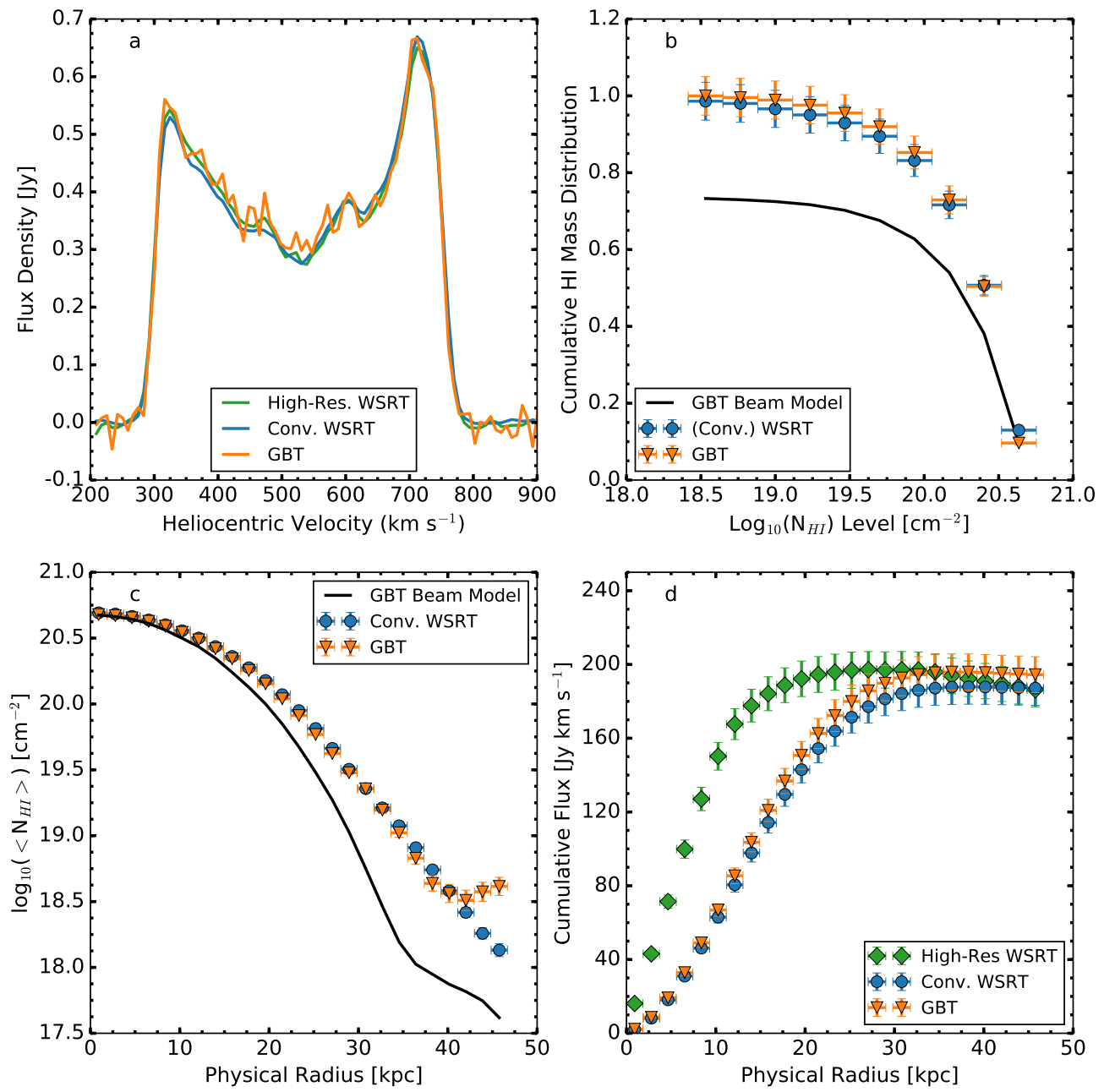

Figure 2.3 Comparison between the high-resolution (green diamonds) WSRT, convolved WSRT (blue circles), and regridded GBT (orange inverted triangles) data sets of NGC891. $a$ : global Hi profile; $b$ : Cumulative Hi mass as a function of $N_{\mathrm{HI}}$. The solid black line simulates an unresolved observation with our GBT beam model; $c$ : projected physical radial dependence on the azimuthally averaged $N_{\mathrm{HI}} ; d$ : projected radial dependence of the cumulative flux. In this case, we also show the results of our analysis on the high-resolution WSRT data. 
Figure 2.3b summarizes the results of the cumulative Hi mass as a function of $N_{\mathrm{HI}}$ for NGC891. While the cumulated Hi mass distribution from the simulated point source observation falls well below the data, values computed from the unmasked GBT and convolved WSRT $N_{\mathrm{HI}}$ images trace each other extremely well within the estimated uncertainties.

Figure $2.3 \mathrm{c}$ and $\mathrm{d}$ show the radial dependence of the mean column density and cumulative flux, respectively. The radial dependence of column density in the GBT and convolved WSRT data are effectively identical within $40 \mathrm{kpc}$, indicating the GBT data do not reveal any large-scale Hi features (down to the Hi column density sensitivity limit) that may potentially related to the substantial extraplanar Hi component and filament observed in NGC891. In Figure 2.3d, the additional flux detected in the high-resolution WSRT data at projected scales smaller than the GBT beam (about $24 \mathrm{kpc}$ at a distance of $9.2 \mathrm{Mpc}$ ) originates from emission that completely fills the smaller WSRT beam while remaining unresolved in the larger GBT beam. Past this point, all three data sets begin to converge to a similar value and profile shape. The dip in cumulative flux in the high-resolution profile highlights the presence of artifacts (e.g., a large-scale negative bowl caused by missing central baselines in the $u-v$ coverage, or residual sidelobes leftover from the deconvolution of the dirty beam). 

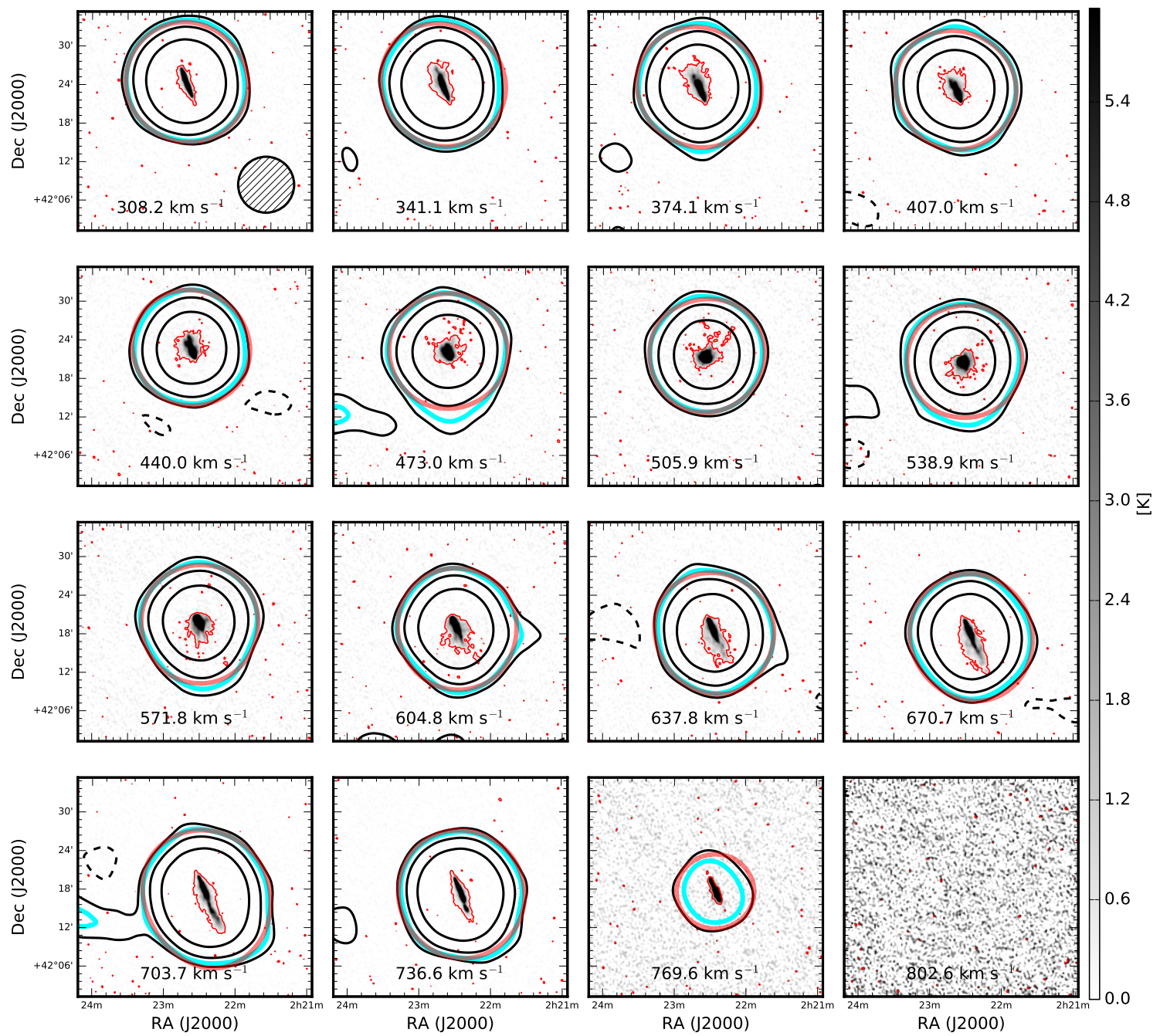

Figure 2.4 Selected channel maps of the NGC891 high-resolution WSRT data cube with corresponding GBT and convolved WSRT contours superimposed. The GBT data are shown in black and cyan contours at levels of -3 (dashed), 3, 5 (thick cyan), and 25 times $0.01 \mathrm{~K}$, or equivalently a column density level of $1.5 \times 10^{17} \mathrm{~cm}^{-2}$ per $8.24 \mathrm{~km} \mathrm{~s}^{-1}$ velocity channel. The grey-scale shows the Hi emission from the WSRT cube. The thin red line denotes a brightness temperature of $0.4 \mathrm{~K}$, or a column density level of $5.9 \times 10^{18} \mathrm{~cm}^{-2}$, and the thick red line denotes emission at $7.5 \times 10^{17} \mathrm{~cm}^{-2}$ (the same level of the cyan contour) in the primary-beam corrected WSRT cube convolved down to the GBT resolution. The GBT beam is shown in the top left panel. 


\subsubsection{The Cumulative Hi Mass vs. $N_{\mathrm{HI}}$}

The channel maps in Figure 2.4 show the extent of the emission detected by the GBT at the $5 \sigma$ level traces the same $5 \sigma$ level in the WSRT data very well. Unlike similar GBT observations of NGC2403 (de Blok et al., 2014a), another galaxy with a large Hi filament, we do not detect any obvious structure associated with the $22 \mathrm{kpc}$ long filament detected in the deep Hi images presented by Oosterloo et al. (2007) in the individual channel maps. The agreement between the various data sets for NGC891 in total flux, mean column density as a function of radius, and the spatial extent of emission in the individual low-resolution data channel maps show the HALOGAS data do an excellent job at recovering the full Hi distribution of NGC891; there is little difference between the HI environment at the $N_{\mathrm{HI}} \sim 10^{18} \mathrm{~cm}^{-2}$ as compared to the $N_{\mathrm{HI}} \sim 10^{19} \mathrm{~cm}^{-2}$ level. The channel maps in Figure 2.4 show the extent of the emission detected by the GBT at the $5 \sigma$ level traces the same $5 \sigma$ level in the WSRT data very well. Unlike similar GBT observations of NGC2403 (de Blok et al., 2014a), another galaxy with a large Hi filament, we do not detect any obvious structure associated with the $22 \mathrm{kpc}$ long filament detected in the deep HI images presented by Oosterloo et al. (2007) in the individual channel maps. The agreement between the various data sets for NGC891 in total flux, mean column density as a function of radius, and the spatial extent of emission in the individual low-resolution data channel maps show the HALOGAS data do an excellent job at recovering the full HI distribution of NGC891; there is little difference between the HI environment at the $N_{\mathrm{HI}} \sim 10^{18} \mathrm{~cm}^{-2}$ as compared to the $N_{\mathrm{HI}} \sim 10^{19} \mathrm{~cm}^{-2}$ level. 


\subsubsection{NGC925}

NGC925 is another galaxy within the HALOGAS survey whose Hi distribution has been thoroughly studied over past decades (e.g., Gottesman 1980; Pisano et al. 1998; Walter et al. 2008; H11). For a galaxy such as NGC925, which has been observed as part of both THINGS and HALOGAS, we can compare the VLA, WSRT, and GBT observations. For this comparison, we utilize the same naturally weighted, residual scaled, blanked cube used to measure the total Hi flux and moment maps in Walter et al. (2008) regridded to the WSRT spatial/spectral scale and convolved to the same angular resolution. Figure 2.5 shows two $N_{\mathrm{HI}}$ images (top and middle left) of NGC925 derived from GBT data. The expanded map shows the companion galaxy, UGC2023. There is no evidence for interacting or connecting material between NGC925 and UGC2023 seen in the GBT data. Figure 2.5 also shows a comparison between the WSRT-HALOGAS (middle right) and VLA-THINGS (top right) data for NGC925 in the form of $N_{\mathrm{HI}}$ images. It is clear that the more sensitive WSRT observations reveal a much more extended Hi distribution than the THINGS data. This large structure $\sim 26 \mathrm{kpc}$ across, which extends from $\alpha_{\mathrm{J} 2000}=02^{h} 24^{m} 30^{s}$, $\delta_{\mathrm{J} 2000}=33^{\circ} 17^{\prime}$ to $\alpha_{\mathrm{J} 2000}=02^{h} 23^{m} 48^{s}, \delta_{\mathrm{J} 2000}=33^{\circ} 12^{\prime}$, is visible in the VLA THINGS data, albeit at very low levels. As the shortest baselines in the THINGS survey are effectively equal to those in HALOGAS at $35 \mathrm{~m}$, we can attribute the additional structure observed by the WSRT strictly to a lower noise floor (as opposed to differences in the maximum recoverable angular scale). Figure 2.5 also shows the velocity field maps computed from the GBT (bottom left) and WSRT (bottom right) data 

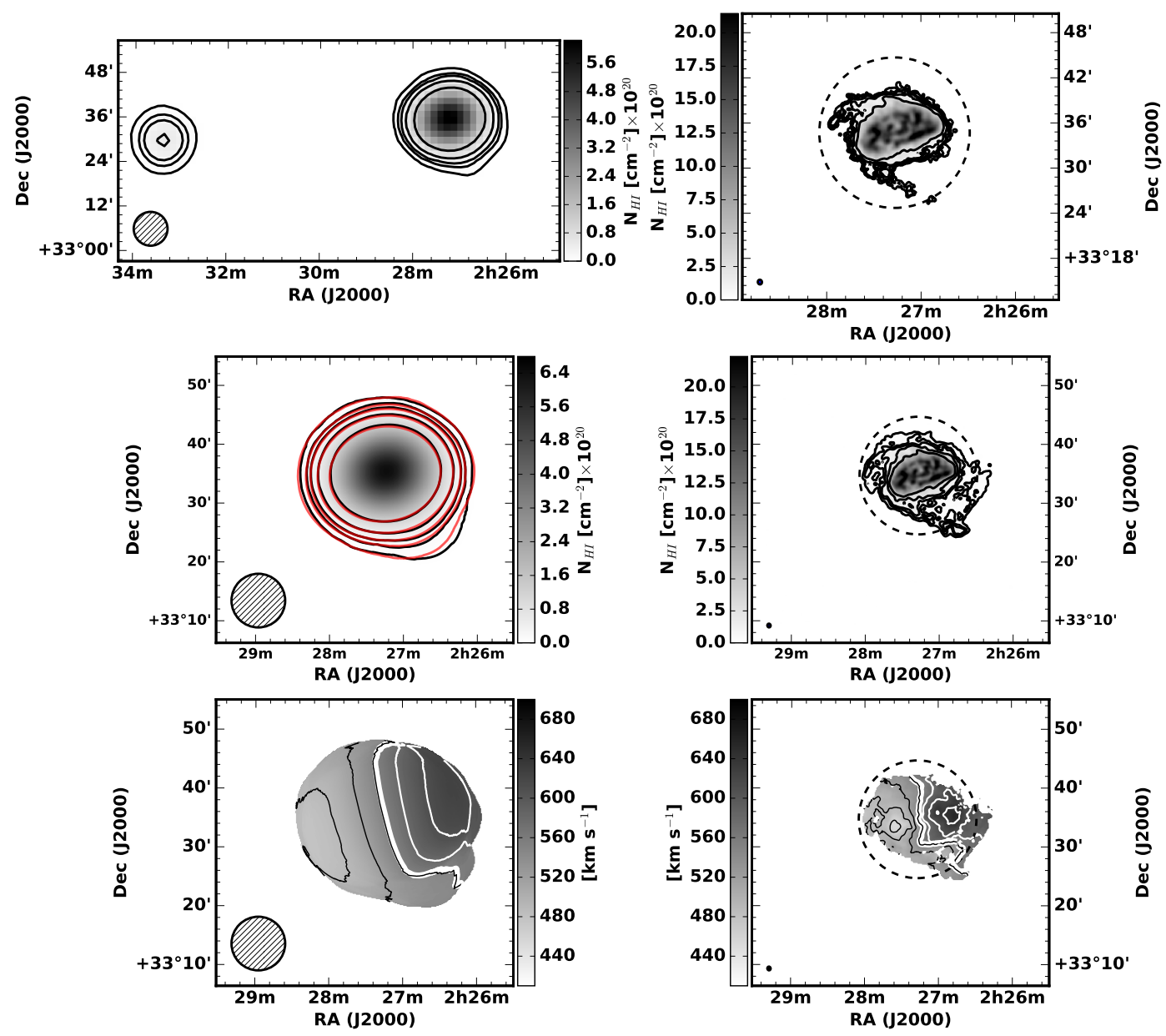

Figure 2.5 A comparison of several data sets for NGC925. The top left panel shows an integrated Hi map of the GBT data for NGC925. UGC2023, a companion to the east of NGC925, is shown in the GBT map to highlight the absence a diffuse Hi bridge connecting the two galaxies. The top right panel shows an $N_{\mathrm{HI}}$ image computed from VLA-THINGS data. The middle and bottom left panels show a zoomed $N_{\mathrm{HI}}$ image of the low resolution cubes data and GBT velocity field. The middle and bottom right panels show the $N_{\mathrm{HI}}$ image derived from the WSRT data and associated velocity field. The red and black contours in the low resolution $N_{\mathrm{HI}}$ images respectively denote WSRT and GBT data; they begin at column density values of $5.0 \times 10^{18} \mathrm{~cm}^{-2}$ and continue at $3,5,10$, and 25 times that level, while the contour levels in the VLA and WSRT $N_{\mathrm{HI}}$ images begin at a $N_{\mathrm{HI}}$ level equivalent to $2.0 \times 10^{19} \mathrm{~cm}^{-2}$ and continue at $3,5,10$, and 25 times that level. The velocity field contours begin at $430 \mathrm{~km} \mathrm{~s}^{-1}$ and continue in steps of $30 \mathrm{~km} \mathrm{~s}^{-1}$. The systemic velocity of $553 \mathrm{~km} \mathrm{~s}^{-1}$ is represented by the thick white line, and the approaching and receding velocities. The dashed circles in the right-hand panels represent the maximum recoverable angular scales of the WSRT and VLA data. 
with the contour levels are given in the Figure caption. The high resolution velocity field shows deviations from axial symmetry coincident the the disturbed structure in the $N_{\mathrm{HI}}$, indicating a deviation from circular rotation. H11 attributes the origin to a possible interaction with a gas-rich dwarf companion seen as a faint enhancement in the Digital Sky Survey optical plates centered at about $\alpha_{\mathrm{J} 2000}=02^{h} 26^{m} 44^{s}$, $\delta=33^{\circ} 25^{\prime} 20^{\prime \prime}$

We show the global HI profiles of NGC925 computed over the same angular area from the GBT, VLA, and the two WSRT data sets in Figure 2.6a. The GBT detects more flux over approaching velocities (approximately $430 \mathrm{~km} \mathrm{~s}^{-1}$ to $550 \mathrm{~km}$ $\mathrm{s}^{-1}$ ) than the WSRT, while both the WSRT and GBT detect more flux over the entire velocity range than what is measured in VLA data.

The difference in the profiles highlights that the recovered flux detected by an interferometer is dependent on the distribution and treatment (e.g., tapering of baseline amplitudes) of the complex visibilities. For this specific THINGS cube, the visibilities were 'naturally' weighted in the AIPS task IMAGR, meaning the visibilities were weighted to maximize surface brightness sensitivity. In the case of NGC925, the WSRT data were designed to maximize both sensitivity (natural same weight) and control over the dirty beam (uniform - density) with the robust parameter set to 0 in the Miriad task, INVERT. An additional 30" Gaussian taper was also applied to the higher spatial frequencies to further maximize sensitivity to faint extended emission. It is therefore a testament to the WSRT observations that approximately $20 \%$ more $\mathrm{HI}$ is detected as compared to the VLA data considering both were optimized to observe extended structure. In addition to the differences 

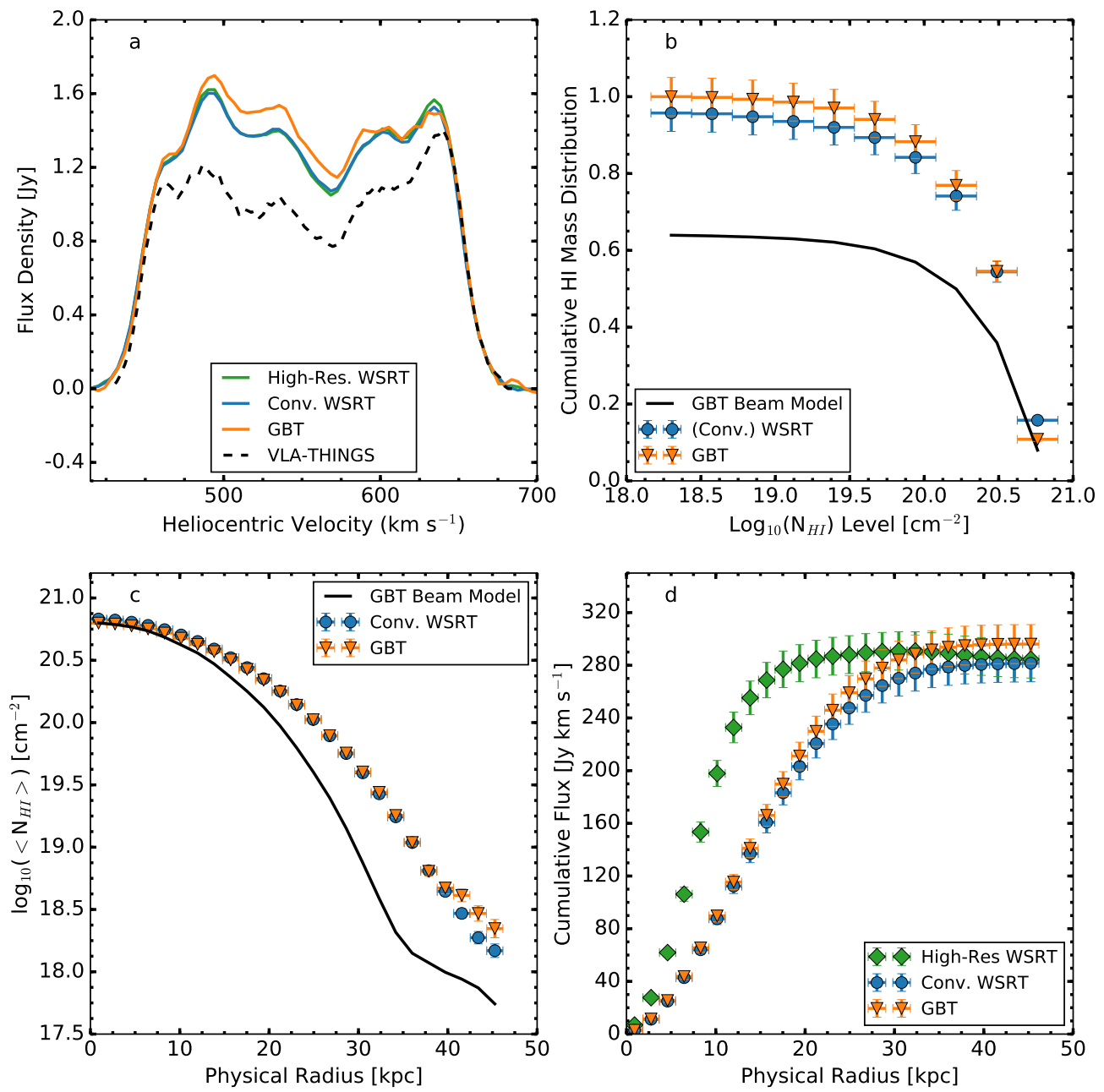

Figure 2.6 Comparison between the high-resolution (green diamonds) WSRT, convolved WSRT (blue circles), and regridded GBT (orange inverted triangles) data sets of NGC925. a: global Hi profile; $b$ : Cumulative HI mass as a function of $N_{\mathrm{HI}}$. The dashed and solid black lines simulate the contribution of a Gaussian beam and our GBT beam model, respectively; $c$ : projected physical radial dependence on the azimuthally averaged $N_{\mathrm{HI}}$; $d$ : projected radial dependence of the cumulative flux. In this case, we also show the results of our analysis on the high-resolution WSRT data. 
in weighting schemes, the antenna positions between the VLA and WSRT cause immutable differences in $u-v$ coverage making direct comparisons in terms of the total flux between these data sets impossible. We can therefore only conclude that, because of the agreement between both high and low-resolution WSRT and GBT flux profiles (which contains the zero-spacings information), there is excellent recovery of the diffuse Hi in the WSRT-HALOGAS data for NGC925.

The cumulative Hi mass as a function of $N_{\mathrm{HI}}$ for NGC925 is presented in Figure 2.6b. Just as is the case with NGC891, the cumulative Hi mass as a function of $N_{\mathrm{HI}}$ measured by the GBT is traced reasonably well by the convolved WSRT data with only slight hints of excess $\mathrm{HI}$ in the $N_{\mathrm{HI}}$ bins below $\log _{10}\left(N_{\mathrm{HI}} / \mathrm{cm}^{2}\right)=$ 20.5. The radial profiles of mean column density and cumulative flux also show consistency within the calculated uncertainties and expected behavior between the high resolution and convolved WSRT data. The slight decrease in the cumulative flux beginning at a projected physical radii $40 \mathrm{kpc}$ also indicates the presence of a negative bowl in the WSRT data.

Selected channel maps of NGC925 from the WSRT-HALOGAS data are shown in greyscale in Figure 2.7 with corresponding contours denoting emission from the GBT and convolved, primary-beam WSRT data overlaid. The GBT shows slightly more extended emission in some velocity channels. A majority of the slight extension corresponds to the velocity range of $500-600 \mathrm{~km} \mathrm{~s}^{-1}$ where the disturbed Hi distribution seen in the high-resolution $N_{\mathrm{HI}}$ images of Figures 2.5 is most prevalent.

Our GBT observations detect $(5.79 \pm 0.29) \times 10^{9} M_{\odot}$ of Hi, while the WSRT data reveal $(5.54 \pm 0.28) \times 10^{9} M_{\odot}$ of Hi measured over the same angular area. H11 

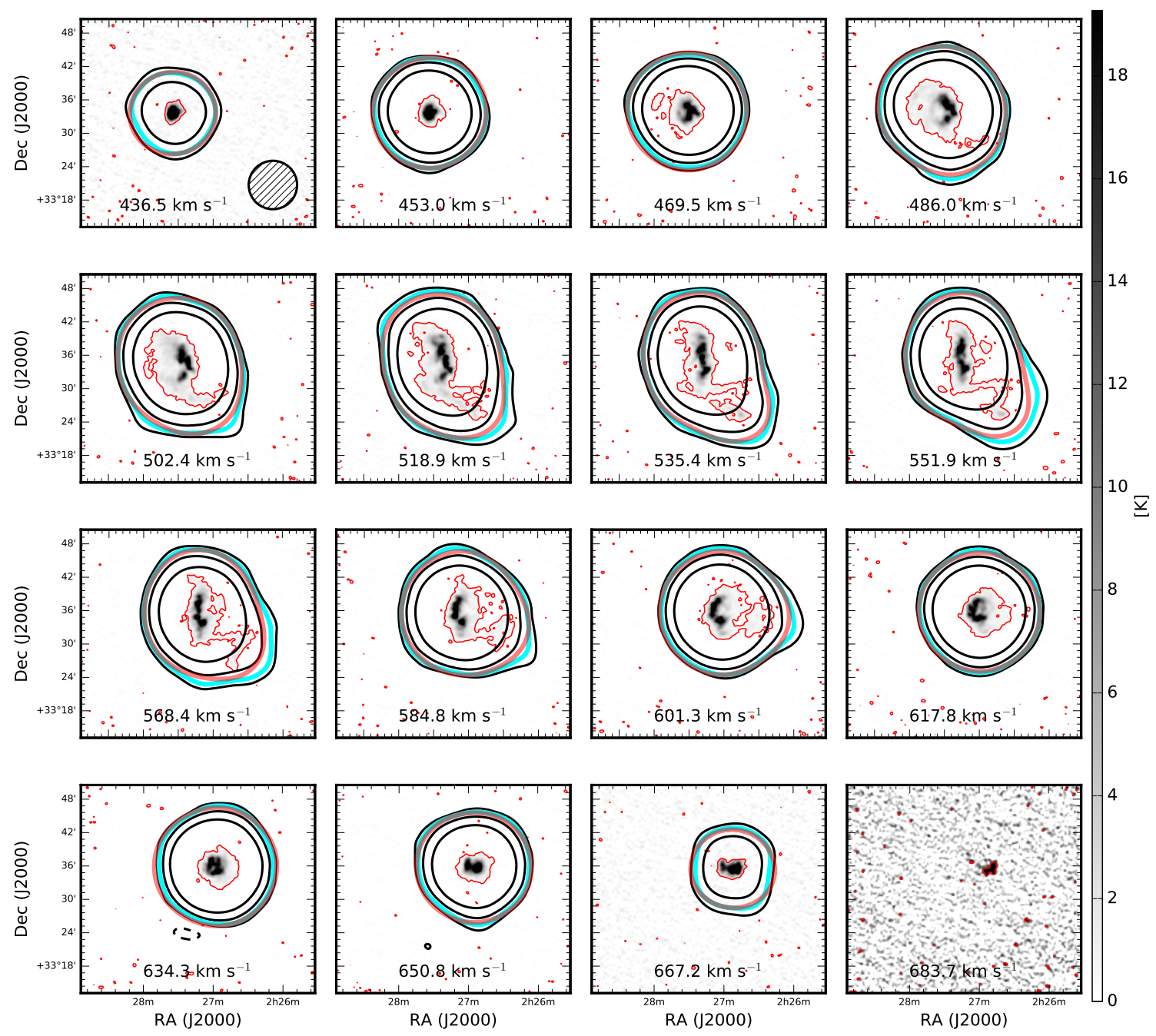

Figure 2.7 Selected channel maps of the NGC925 WSRT data cube with corresponding GBT channel maps superimposed. The GBT data are shown in black and cyan contours at levels of -3 (dashed), 3, 5 (thick cyan), and 25 times $0.01 \mathrm{~K}$, or equivalently a column density level of $9.75 \times 10^{16} \mathrm{~cm}^{-2}$ per $4.12 \mathrm{~km} \mathrm{~s}^{-1}$ velocity channel. The grey-scale shows the Hi emission from the WSRT cube. The thin red line denotes a brightness temperature of $0.36 \mathrm{~K}$, or a column density levels at $2.70 \times 10^{18}$ $\mathrm{cm}^{-2}$, and the thick red line denotes emission at $4.88 \times 10^{18} \mathrm{~cm}^{-2}$ (the same level of the cyan contour) in the primary-beam corrected WSRT cube convolved down to the GBT resolution. The GBT beam is shown in the top left panel. 


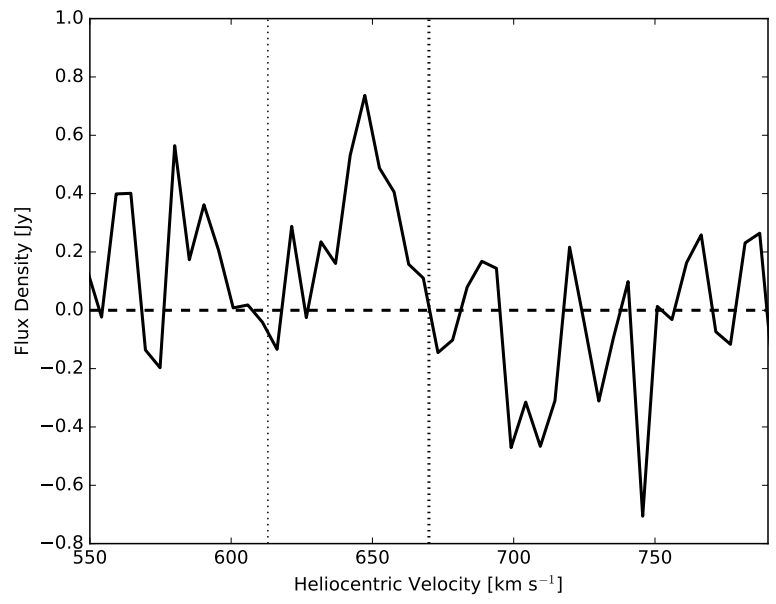

Figure 2.8 Global Hi profile derived from GBT data for the northern companion of NGC925 deemed 'Halogas' by Karachentsev \& Kaisina (2013).

found faint emission extending towards the systemic velocity (see their Figure 6). This "beard" gas is interpreted as a slowly rotating halo seen in projection against the disk with a total Hi mass on the order of $10^{8} M_{\odot}$. While slight in magnitude, there is some emission picked up by the GBT which was missed in the original HALOGAS observations. The GBT observations therefore reveal additional HI in NGC925 that must be some combination of extended and diffuse as it was not detected in neither optimally weighted VLA data nor in the more sensitive WSRT data. We defer correcting the WSRT data for missing short spacings for a future paper; nevertheless, a high-resolution cube that recovers emission at angular scales will provide an excellent data set for a detailed dynamical study relating the beard gas to the extraplanar component.

H11 also noted the presence of the a small companion centered to the North at about $\alpha_{\mathrm{J} 2000}=02^{h} 27^{m} 20^{s}, \delta=33^{\circ} 57^{\prime} 30^{\prime \prime}$ in the velocity range of $613-665 \mathrm{~km} \mathrm{~s}^{-1}$. This companion was cataloged as 'Halogas' by Karachentsev \& Kaisina (2013). The 
companion has a total Hi mass (as measured by the GBT) of $(3.11 \pm 0.15) \times 10^{7} M_{\odot}$ consistent with the measurement from H11. A global Hi profile taken from the GBT data is shown in Figure 2.8; there is no detection of a bridge of Hi between Halogas and NGC925.

\subsubsection{NGC4414}

NGC4414 is a moderately inclined $\left(i=50^{\circ}\right)$ SAc galaxy, and is one of the most

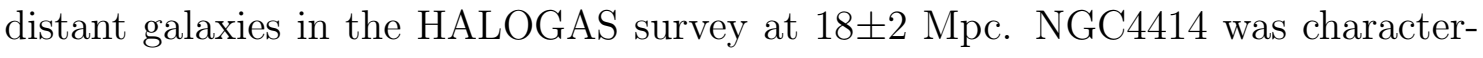
ized through tilted ring fitting (de Blok et al., 2014b) as having a regular rotating inner disk within $240^{\prime \prime}(\sim 21 \mathrm{kpc}$ at the distance of NGC4414) in radius and an outer disk that is mostly dominated by rotation with some evidence for radial and noncircular motions towards the edge of the extended Hi distribution. The high-resolution WSRT-HALOGAS observations do not show any evidence for an interaction besides the disturbed outer disk, though NGC4414 likely has undergone some weak interaction with neighboring galaxies within the Coma I cluster in the past. de Blok et al. (2014b) mention a possible source of an interaction may be the small galaxy, SDSS J122646.27+311904.8, but neither the WSRT or GBT detect any HI at its position.

A comparison between the GBT and WSRT data is shown in Figure 2.9 in the form of integrated Hi intensity and velocity field maps. As noted by de Blok et al. (2014b), the inner regions of the velocity field show very well-behaved rotation in the inner regions of the galaxy with motions deviating from circular towards the edge of the Hi disk. These areas of irregular rotation overlap well with the presence of the 

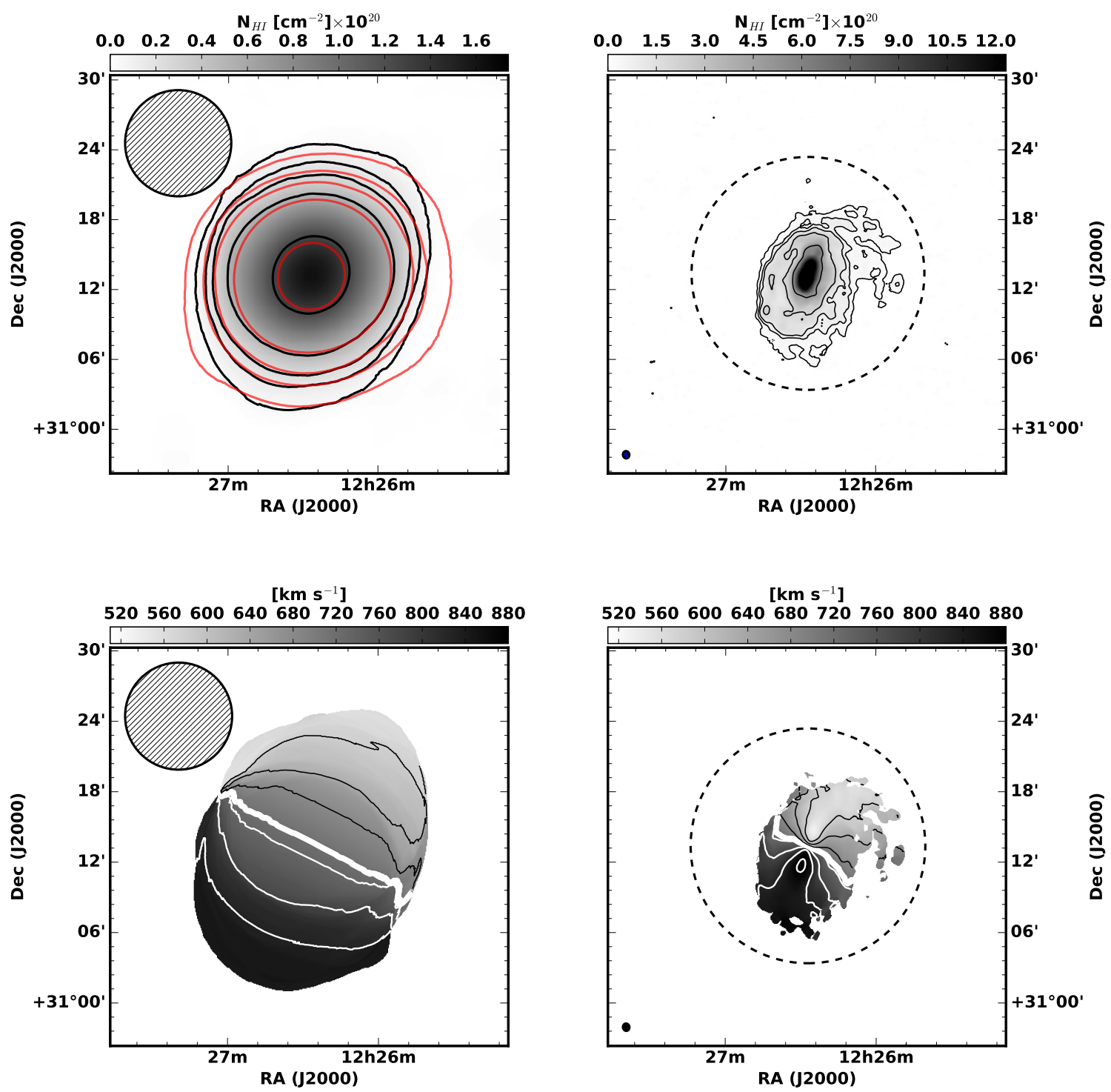

Figure 2.9 A comparison of the $N_{\mathrm{HI}}$ images (top) and velocity fields (bottom) of NGC4414 derived from the GBT (left) and WSRT-HALOGAS (right) data. The red and black contours in the low resolution $N_{\mathrm{HI}}$ images respectively denote WSRT and GBT data; they begin at a $N_{\mathrm{HI}}$ level of $5.0 \times 10^{18} \mathrm{~cm}^{-2}$ and increase by factors of $3,5,10$, and 25. The contours in the high-resolution WSRT $N_{\mathrm{HI}}$ image begin at a $N_{\mathrm{HI}}$ level of $2.0 \times 10^{19} \mathrm{~cm}^{-2}$ and increase by factors of $3,5,10$, and 25 . The velocity contours in both maps begin at $530 \mathrm{~km} \mathrm{~s}^{-1}$ and increase by $50 \mathrm{~km} \mathrm{~s}^{-1}$ with the systemic velocity of NGC4414 of $716 \mathrm{~km} \mathrm{~s}^{-1}$ represented by the thick white contour. The receding and approaching sides are denoted by white and black contours, respectively. The GBT (WSRT) beams are shown for their respective data sets in the top (bottom) left corner of each panel. The dashed circles in the right-hand panels represent the maximum recoverable angular scales of the WSRT data. 

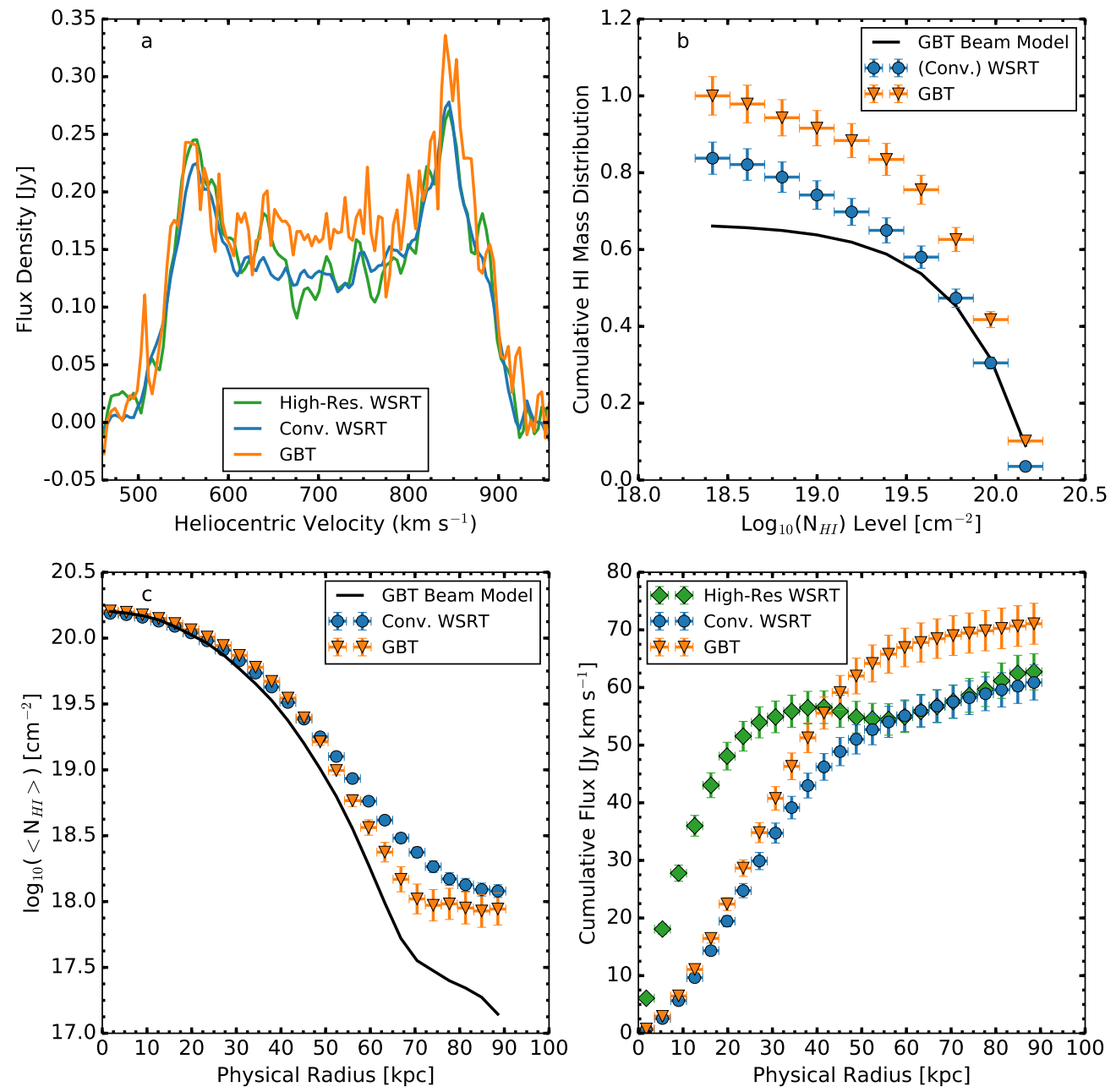

Figure 2.10 Comparison between the high-resolution (green diamonds) WSRT, convolved WSRT (blue circles), and regridded GBT (orange inverted triangles) for NGC4414. $a$ : global Hi profile; $b$ : Cumulative Hi as a function of $N_{\mathrm{HI}}$. The dashed and solid black lines simulate the contribution of a Gaussian beam and our GBT beam model, respectively; $c$ : projected physical radial dependence on the azimuthally averaged $N_{\mathrm{HI}}$; $d$ : projected radial dependence of the cumulative flux. In this case, we also show the results of our analysis on the high-resolution WSRT data. 

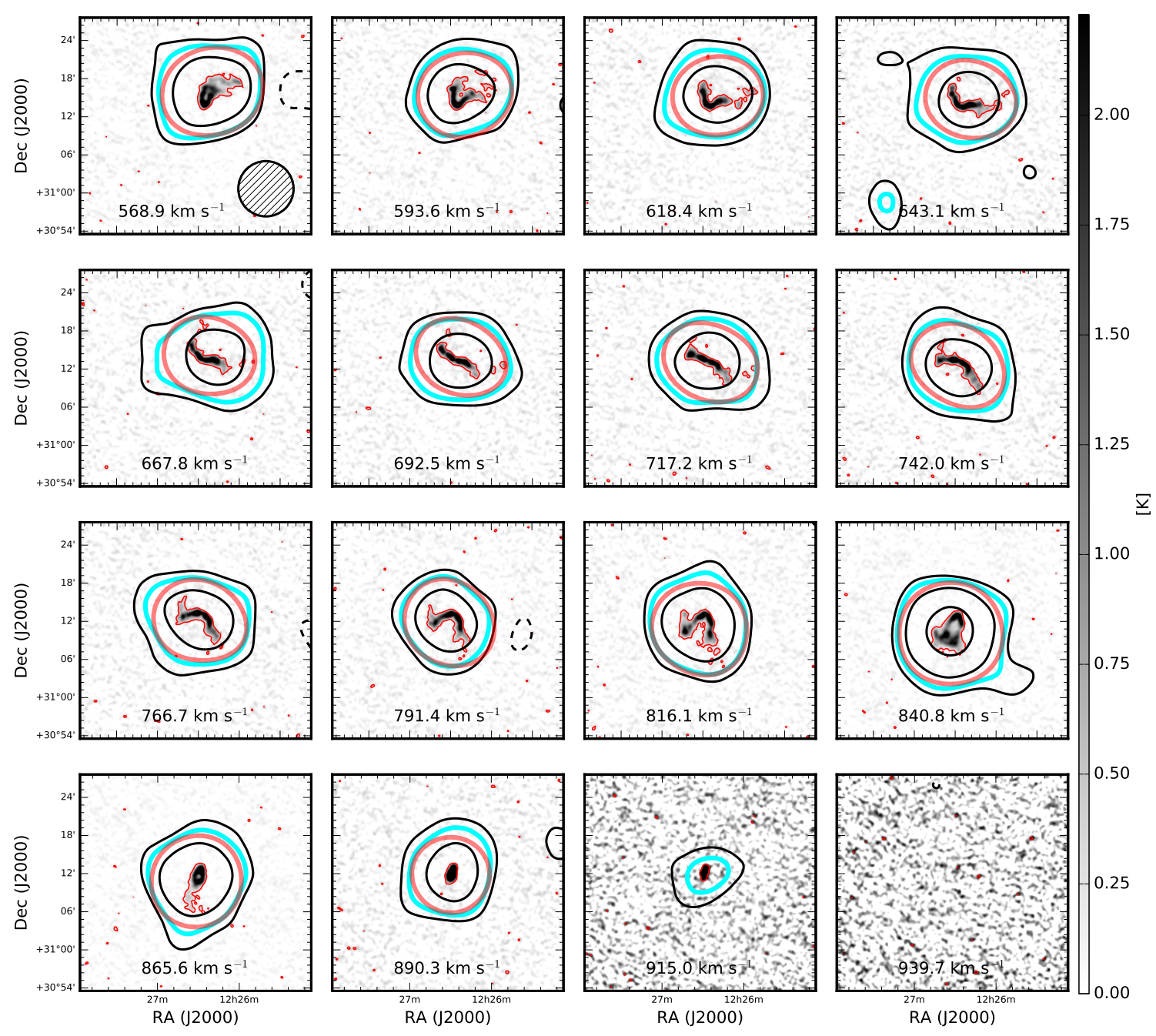

Figure 2.11 Selected channel maps of the NGC4414 WSRT data cube with corresponding GBT channel maps superimposed. The GBT data are shown in black and cyan contours at levels of -3 (dashed), 3, 5 (thick cyan), and 25 times $0.01 \mathrm{~K}$, or equivalently a column density level of $9.75 \times 10^{16} \mathrm{~cm}^{-2}$ per $4.12 \mathrm{~km} \mathrm{~s}^{-1}$ velocity channel. The grey-scale shows the HI emission from the WSRT cube. The thin red line denotes a brightness temperature of $0.39 \mathrm{~K}$, or a column density levels at $2.92 \times 10^{18}$ $\mathrm{cm}^{-2}$, and the thick red line denotes emission at $2.93 \times 10^{17} \mathrm{~cm}^{-2}$ (the same level of the cyan contour) in the primary-beam corrected WSRT cube convolved down to the GBT resolution. The GBT beam is shown in the top left panel. 
disturbed structure seen in the high resolution high-resolution WSRT $N_{\mathrm{HI}}$ image, which again, may be evidence for a past interaction. The extent of the overall Hi distribution is within the maximum recoverable angular scale as denoted by the dashed circles.

The global flux density profiles of NGC4414 derived from the GBT and WSRT data are shown in the top left panel of Figure 2.10. The GBT detects more flux overall, and excess flux is encountered over almost the entire velocity range. The total Hi mass measured by the GBT is $(5.43 \pm 0.27) \times 10^{9} M_{\odot}$, which is $\sim 1 \times 10^{9} M_{\odot}$ more HI than is measured in the WSRT data over the same area. The large offset in the fluxes may be due, in part, to a large plume of Hi extension to the West, which is further evidence for a past interaction.

The cumulative Hi mass as a functions of $N_{\mathrm{HI}}$ calculated from both data sets for NGC4414 are summarized in the top right panel. The convolved WSRT data begin to trace the simulated unresolved observation quite well past $\log _{10}\left(N_{\mathrm{HI}} / \mathrm{cm}^{2}\right)$ $=19.0$. The offset of $\sim 15 \%$ between the two data profiles at the lower $N_{\mathrm{HI}}$ bins indicates that the WSRT observations may resolve out structure that extends past the maximum recoverable angular scale with a peak $N_{\mathrm{HI}}$ on the order of $\sim 10^{1} 8 \mathrm{~cm}^{-2}$.

The radial dependent properties are shown in the lower two panels of Figure 2.10. The azimuthally averaged $N_{\mathrm{HI}}$ profiles interestingly begin to differ at projected physical of about $60 \mathrm{kpc}$, while a prevalent dip in the cumulative flux profiles of the WSRT data begins near $45 \mathrm{kpc}$. Considering the $N_{\mathrm{HI}}$ profiles are derived from masked $N_{\mathrm{HI}}$ images, the deviation between the GBT and WSRT profiles could reflect the presence of artifacts, such as leftover residual sidelobes from 
deconvolution, that increase noise properties at larger angular extents to mimic the presence of legitimate signal. The variations in the WSRT cumulative flux profiles explicitly demonstrate the presence of artifacts in these data cubes.

Channel maps of NGC4414 similar to Figures 2.4 and 2.7 are presented in Figure 2.11. The outermost $3 \sigma$ contour generally extends past the $5 \sigma$ contour of the convolved WSRT data in each selected velocity channel. This is consistent with the behavior of the global Hi profile from Figure 2.10. The channel maps reveal the extent of the NGC4414 emission in a single velocity channel is within the angular sensitivity limit $\left(\sim 20^{\prime}\right.$, or $\sim 100 \mathrm{kpc}$ at the distance of NGC4414) in the WSRT data. Additionally, the angular extent of NGC4414 is only a few times the GBT beam area as evidence by the steep drop in the cumulative Hi mass function. Considering all these factors, we conclude that a majority of the excess emission detected by the GBT is likely due to artifacts (e.g., residual sidelobe structure) in the WSRT cubes, as opposed to resolved out structure.

de Blok et al. (2014b) noted in their analysis that gas at velocities lower than the local rotational velocity (i.e., "beard" gas) is present in both the receding and approaching sides of the rotation curve for the inner disk of NGC4414, and should be interpreted as extraplanar gas in the inner disk. Through a variety of techniques including blanking high column density pixels and subtracting Gaussian fits of the velocity profiles within the observed data cube (Fraternali et al., 2002), de Blok et al. (2014b) determined the Hi mass of the extraplanar gas associated with the inner disk to be between 2 and 6.5 percent of the total Hi mass of the inner disk (i.e., about $\left.2 \times 10^{8} M_{\odot}\right)$. Similarly, the authors concluded the extraplanar gas associated 
with the outer disk to be about 1 to 2 percent of the total Hi mass of the system. In the future, merging the GBT and WSRT data sets will provide an excellent basis for characterizing dynamical links between anomalous velocity gas and an extraplanar component in NGC4414 as well as characterizing the kinematics of a potential past interaction.

\subsubsection{NGC4565}

NGC4565 is a large, edge-on SAb galaxy with two very nearby companion galaxies: IC 3571 directly to the north and NGC 4562 to the southwest; only NGC 4562 is partially resolved in our GBT data. The apparent connection is due to the beam confusion stemming from the large GBT beam. The SFR for NGC4565 is among the lowest in the entire HALOGAS sample at $0.67 M_{\odot} \mathrm{yr}^{-1}$. The $N_{\mathrm{HI}}$ images and velocity fields derived from both the GBT and WSRT data sets are detailed in Figure 2.12. These specific HALOGAS observations for NGC4565 have been discussed by Zschaechner et al. (2012) and expose a potential interaction between IC3571 and NGC4565 as evidenced by the possible tidal material shown as a separate cloud complex between IC3571 and the disk of NGC4565 in the top right panel of Figure 2.12. It remains unclear if this structure is related to tidal interactions between the main disk of NGC4565 and IC3571, or an accretion process.

The total Hi measured in the GBT data over the same angular region as the WSRT data (including companions) is $(7.46 \pm 0.39) \times 10^{9} M_{\odot}$, while the primary-beam corrected WSRT data convolved to the GBT resolution gives $(7.32 \pm 0.37) \times 10^{9} M_{\odot}$. 

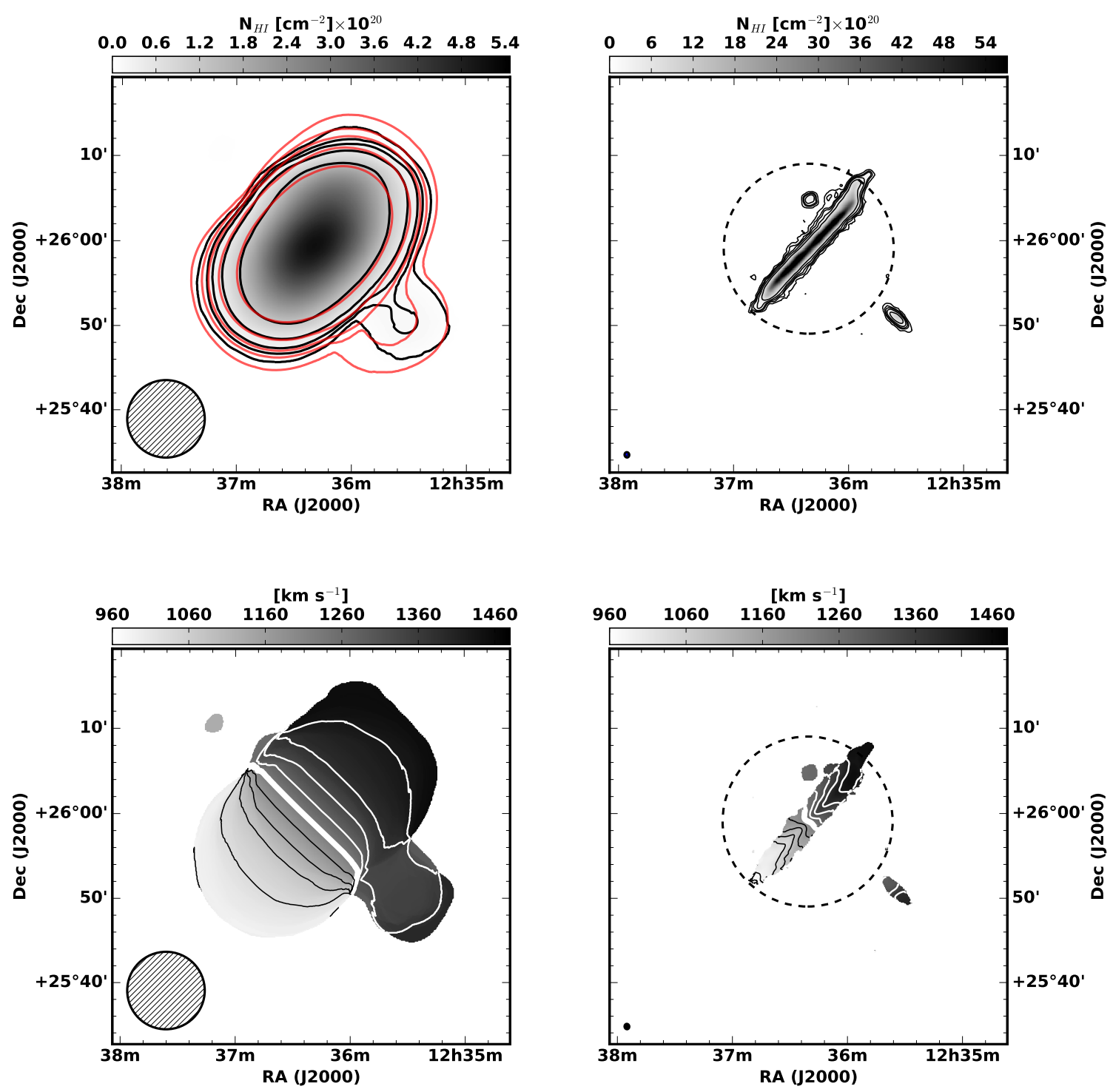

Figure 2.12 $N_{\mathrm{HI}}$ images (top) and velocity fields (bottom) for GBT (left) and WSRT (right) data for NGC4565. The red and black contours in the low resolution $N_{\mathrm{HI}}$ images respectively denote WSRT and GBT data. The contours start at a column density value of $5 \times 10^{18}$ and continue at 3, 5, 10, and, 25 times that level in the GBT image. The contours in the associated high-resolution WSRT image begin at a level equivalent to $2.0 \times 10^{19} \mathrm{~cm}^{-2}$ and continue at $3,5,10$, and 25 times that level. The contours in both velocity fields begin at $970 \mathrm{~km} \mathrm{~s}^{-1}$ and continue in steps of 50 $\mathrm{km} \mathrm{s}^{-1}$. The systemic velocity of $1230 \mathrm{~km} \mathrm{~s}^{-1}$ is represented by the thick line, and the approaching and receding velocities are denoted by black and white contours, respectively. The dashed circles in the right-hand panels represent the maximum recoverable angular scales of the WSRT data. 

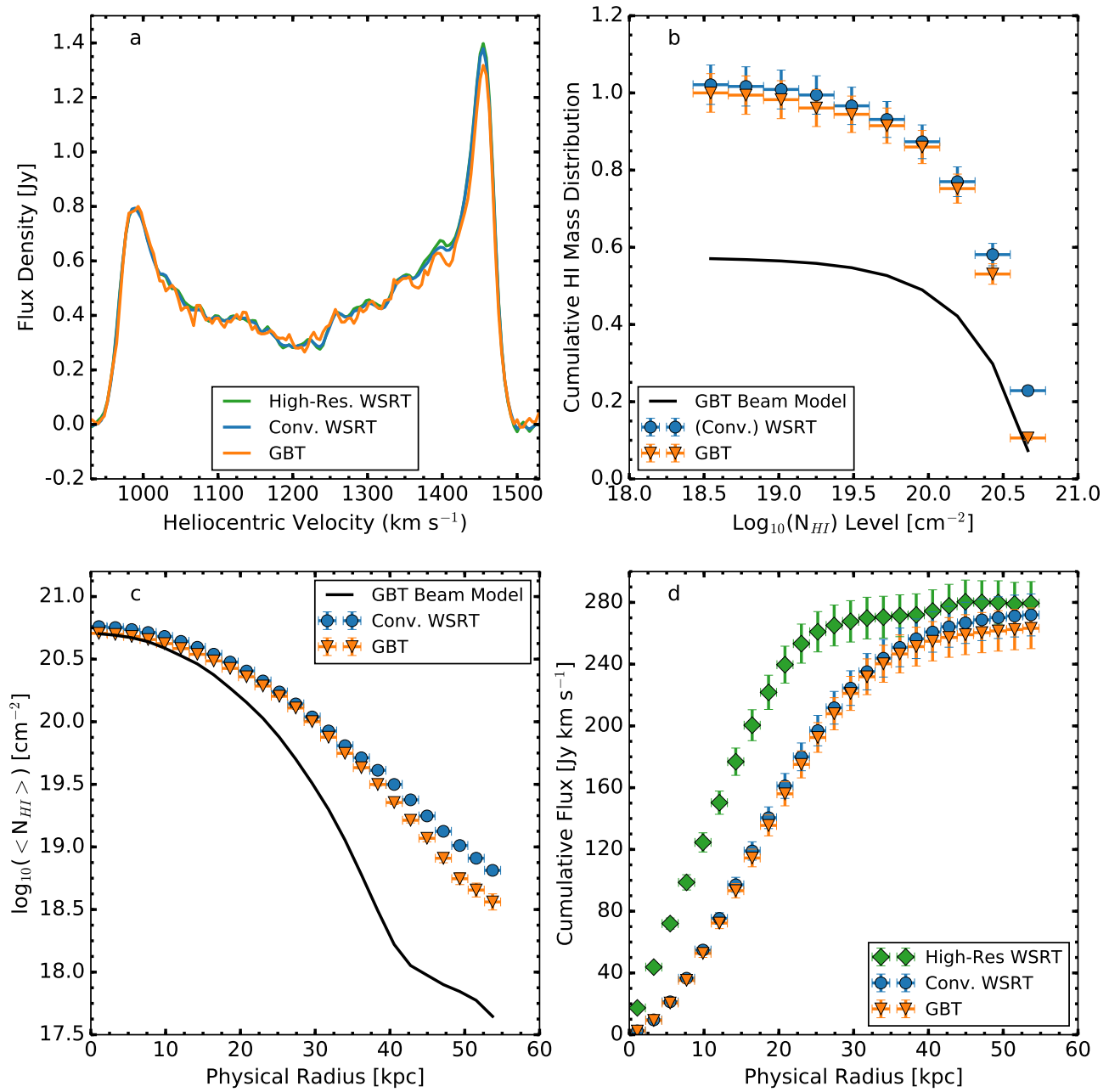

Figure 2.13 Comparison between the high-resolution (green diamonds) WSRT, convolved WSRT (blue circles), and regridded GBT (orange inverted triangles) for NGC4565. $a$ : global Hi profile; $b$ : Cumulative Hi mass as a function of $N_{\mathrm{HI}}$. The dashed and solid black lines simulate the contribution of a Gaussian beam and our GBT beam model, respectively; $c$ : projected physical radial dependence on the azimuthally averaged $N_{\mathrm{HI}}$; $d$ : projected radial dependence of the cumulative flux. In this case, we also show the results of our analysis on the high-resolution WSRT data. 

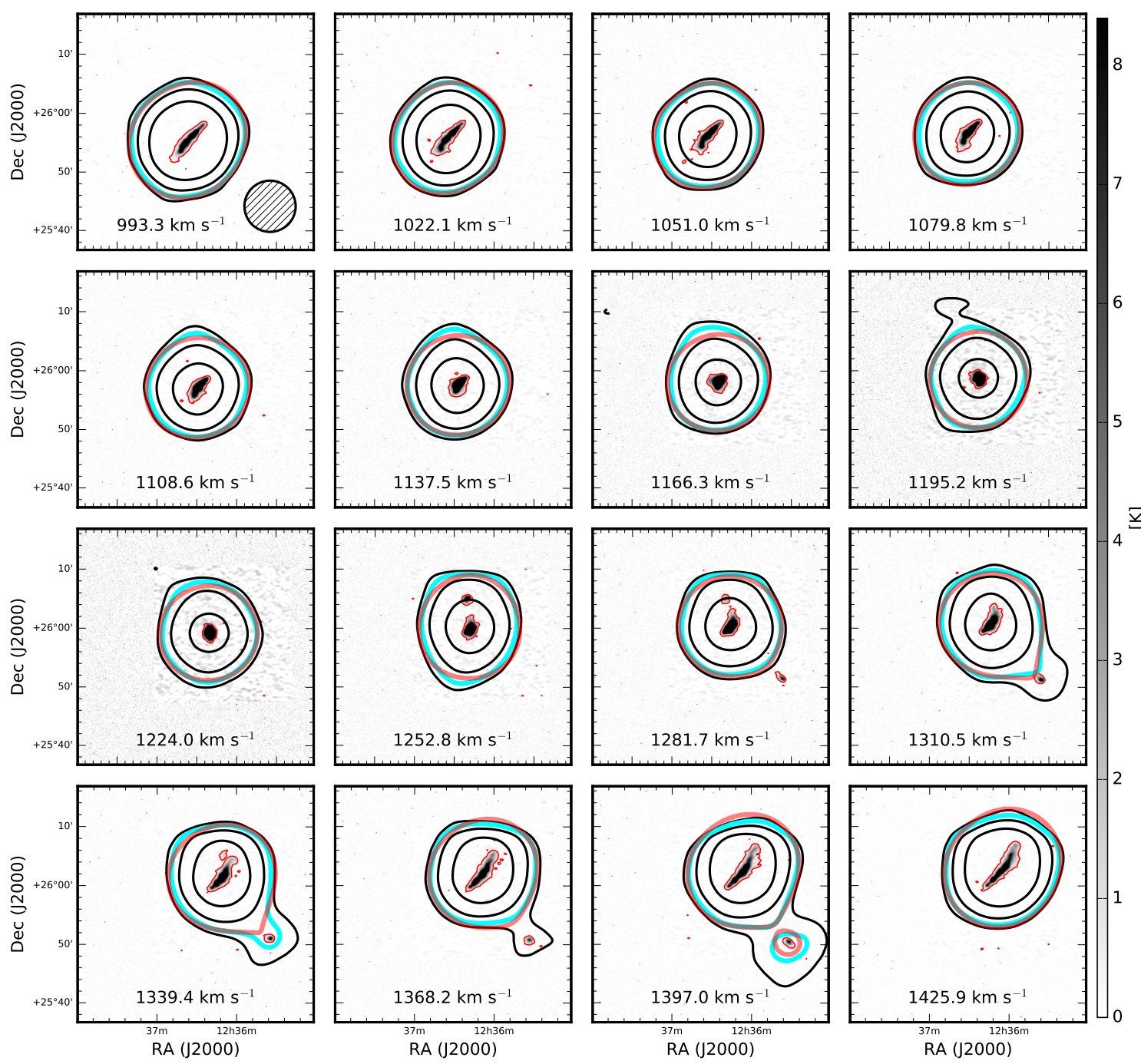

Figure 2.14 Selected channel maps of the NGC4565 WSRT data cube with corresponding GBT channel maps superimposed. The GBT data are shown in black and cyan contours at levels of -3 (dashed), 3, 5 (thick cyan), and 25 times $0.01 \mathrm{~K}$, or equivalently a column density level of $1.12 \times 10^{17} \mathrm{~cm}^{-2}$ per $4.12 \mathrm{~km} \mathrm{~s}^{-1}$ velocity channel. The grey-scale shows the HI emission from the WSRT cube. The thin red line denotes a brightness temperature of $0.54 \mathrm{~K}$, or a column density levels at $4.05 \times 10^{18}$ $\mathrm{cm}^{-2}$, and the thick red line denotes emission at $5.60 \times 10^{17} \mathrm{~cm}^{-2}$ (the same level of the cyan contour) in the primary-beam corrected WSRT cube convolved down to the GBT resolution. The GBT beam is shown in the top left panel. 
The Hi profiles are shown in Figure 2.13 and match extremely well.

The cumulative HI mass as a function of $N_{\mathrm{HI}}$ for NGC4565 is presented in Figure 2.13b. Both distributions also trace each other remarkably well within statistical uncertainties. Additionally, the excellent agreement between all NGC4565 data sets extend to the mean radial column density/cumulative flux. The slight offsets towards higher projected physical radii can be attributed to a residual baseline structure in the GBT cube.

Selected channels are shown in Figure 2.14 with the GBT and WSRT superimposed on the high-resolution WSRT channels. The $5 \sigma$ level of emission detected from the GBT is consistent with the same level in the convolved WSRT data.

The consistency in the various profiles of Figure 2.13 and contours in Figure 2.14 indicate there is no extended diffuse Hi reservoir around NGC4565 at the $N_{\mathrm{HI}}$ levels of $\sim 10^{18} \mathrm{~cm}^{-2}$. Zschaechner et al. (2012) also did not find evidence using the same high resolution WSRT data of any significant amount of extraplanar HI. The authors of that study proposed this absence is due to no significant disk-halo cycling of material in NGC4565. The lack of any sort of extended low column density Hi component supports this conclusion.

\subsection{Discussion}

In this section we will first discuss our initial survey results in the context of future Hi surveys planned for forthcoming radio telescopes. The second subsection explores methods to link the diffuse environment of the HALOGAS galaxies to 
intrinsic galaxy properties and thus the theoretical predictions from simulations.

\subsubsection{Implications for Future Hi Surveys}

It is well known through a combination of models (e.g., Maloney 1993; Dove \& Shull 1994; Bland-Hawthorn et al. 2017) and observations (van Gorkom, 1996) that the transition from optically-thick to optically-thin medium decreases the efficiency of self-shielding leading to a dramatic drop in the fraction of $\mathrm{HI}$ to total hydrogen (i.e., neutral fraction) primarily due to UV and X-Ray background radiation. This effect is demonstrated in cosmological simulations of the Hi distribution (e.g., Popping et al. 2009; Rahmati et al. 2015; Marinacci et al. 2017); in particular, Popping et al. (2009) shows the neutral fraction drops from unity to about a percent between $18.0<\log _{10}\left(N_{\mathrm{HI}} / \mathrm{cm}^{2}\right)<20.0$. This rapid decrease results in a plateau in the HI distribution function that effectively predicts the probability of detecting substantial Hi structures at $N_{\mathrm{HI}} \sim 10^{18} \mathrm{~cm}^{-2}$ is very low even with deep surveys. The flux as a function of velocity, cumulative HI mass as a function of $N_{\mathrm{HI}}$, and azimuthally averaged profiles of column density and cumulative flux show that, overall, the WSRT observations do an excellent job of recovering the low level HI. Save for NGC4414, we see that the HI distributions do not significantly differ between $N_{\mathrm{HI}} \sim 10^{18} \mathrm{~cm}^{-2}$ and $\sim 10^{19} \mathrm{~cm}^{-2}$.

The dearth of diffuse Hi around these sources has intriguing implications on both the search for cold flow accretion and the determination of the edge of disk gas in late-type galaxies. Many simulations (e.g., Kereš et al. 2005, 2009; Birnboim \& 
Dekel 2003) have predicted that less massive galaxies at low redshift will replenish gas through the form of cold filaments which penetrate the halo down to the disk edge where some fraction of the gas should cool sufficiently to be neutral. Aside from characterizing gas accretion mechanisms, deep Hi observations are also useful for tracing large-scale structure in the local Universe. Simulations undertaken by Nuza et al. (2014) predict M31 and Milky Way analogues to sit within a clumpy, yet very diffuse neutral component with Hi masses on the order of $\sim 10^{8} M_{\odot}$ existing predominately above $N_{\mathrm{HI}} \sim 10^{17} \mathrm{~cm}^{-2}$. Our cumulative Hi mass as a function of $N_{\mathrm{HI}}$ plots are consistent with this prediction. On average, only $2 \%$ of the total HI mass exists in column densities below $1 \times 10^{19} \mathrm{~cm}^{-2}$ as measured in the lowresolution data sets (see Section 2.5.2). Given the results of Popping et al. (2009), in which the HI surface area distribution function is predicted to steepen at $N_{\mathrm{HI}}$ levels lower than $10^{18} \mathrm{~cm}^{-2}$, we expect the offset between the GBT and WSRT cumulative Hi distributions would widen considerably with the detection of diffuse Hi that traces the large scale structure of the cosmic web, an extended reservoir of diffuse neutral Hi, and/or cold flow filaments at the $10^{18} \mathrm{~cm}^{-2}$ column density level. Such structures would likely be resolved out in an interferometric data set and therefore not contribute at all to the cumulative Hi mass at these low $N_{\mathrm{HI}}$ levels.

Detailed analysis of the high-resolution HALOGAS Hi data by E. Jütte (2018; in preparation) reveal only a few newly detected Hi clouds which may be accreting. Furthermore, maps produced by Wolfe et al. (2016) of the apparent connection between M31 and M33 original discovered by Braun \& Thilker (2004), which go several times deeper in column density than our observations, reveal only discrete 
clouds of Hi with masses on the order of $\sim 10^{5} M_{\odot}$ as opposed to a continuous, smooth diffuse component. These early high-resolution results coupled with the small amount of newly detected Hi in the initial GBT data suggest that, if these galaxies are currently accreting gas, most of it must be ionized and unobservable in HI emission. The absence of significant Hi structure at the $N_{\mathrm{HI}}$ level of $10^{18} \mathrm{~cm}^{-2}$ supports the notion that Hi surveys of external galaxies must go at least as deep as $N_{\mathrm{HI}} \sim 10^{17} \mathrm{~cm}^{-2}$ to substantially increase the probability of detecting emission associated with the IGM and/or cold mode accretion.

\subsubsection{The Relationship Between Diffuse Hi and Cold Mode Accretion}

While the GBT data do not reveal new features below the $N_{\mathrm{HI}} \sim 10^{18} \mathrm{~cm}^{-2}$ level, as the remaining data are analyzed it is still important to consider the implications of potential diffuse features and their relation to cold flows as predicted from simulations. Namely, how would diffuse Hi related to cold mode accretion manifest itself in emission? Does the diffuse gas originate from outflows, tidal interactions with nearby companions, or accretion from the IGM?

To explore the relationship between the diffuse Hi around nearby galaxies and simulation predictions, we measure the diffuse Hi mass fraction as defined by

$$
f_{19}=1-\frac{M_{19}}{M_{\mathrm{HI}}}
$$

where $M_{\mathrm{HI}}$ is the total Hi mass detected for that particular telescope (taken from pixels with $N_{\mathrm{HI}}$ values at and above the $3 \sigma$ detection limit listed in Table 2.2 to avoid 

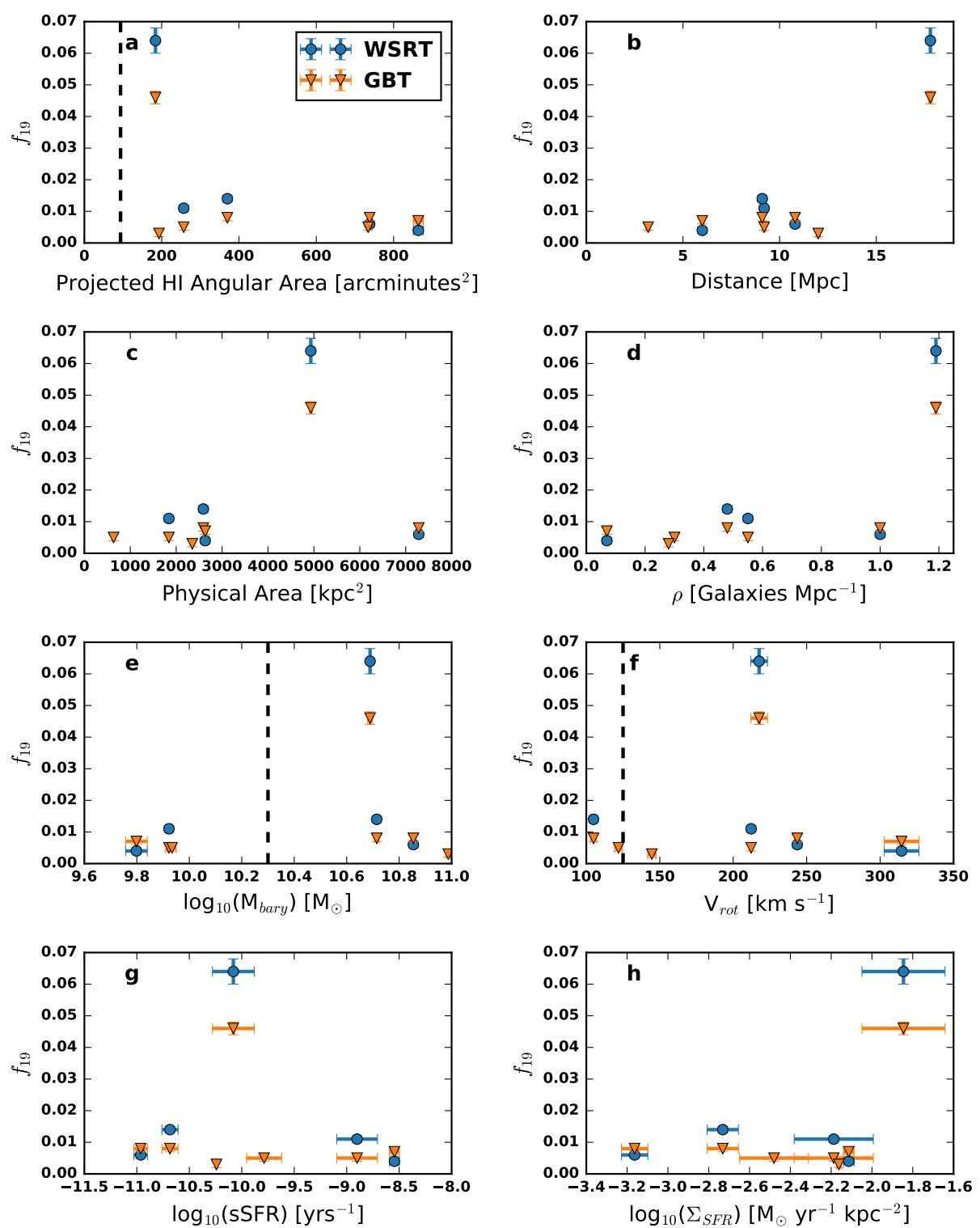

Figure 2.15 Environmental and intrinsic property relations with excess Hi ratio, $f_{19}$. a: $f_{19}$ as a function of projected angular area. The vertical dashed line marks the area of the GBT beam (up to the FWHM); b: $f_{19}$ as a function of distance; c: $f_{19}$ as a function of projected physical area; $\mathrm{d}$ : $f_{19}$ as a function of galaxy density. $\rho$ is taken from Tully (1988); e: $f_{19}$ as a function of the source galaxy baryonic mass $\left(\mathrm{M}_{\mathrm{HI}}+\right.$ $\mathrm{M}_{*}$ ). The vertical dashed line corresponds to the baryonic mass threshold from Kereš et al. (2005) below which cold mode accretion is the dominant accretion mechanism; $\mathrm{f}$ : $f_{19}$ as a function of rotational velocity. The vertical dashed line corresponds to the threshold mass at which galaxies should be gas dominated (Kannappan et al., 2013); g: $f_{19}$ as a function of specific SFR (sSFR); h: $f_{19}$ as a function of the surface density of star formation $\left(\Sigma_{S F R}\right)$. 
measuring into the noise) and $M_{19}$ is the HI mass above the $N_{\mathrm{HI}}$ level of $1 \times 10^{19}$ $\mathrm{cm}^{-2}$ in the unmasked GBT and convolved WSRT $N_{\mathrm{HI}}$ images.

The purpose of this parameter is to measure the mass fraction of diffuse $\mathrm{HI}$ associated with a given galaxy. We can then relate the presence (or dearth) of diffuse Hi to properties predicted by cosmological simulations to correlate with higher rates of cold mode accretion. In the context of our subsequent discussion, we define diffuse Hi to firstly be below the $N_{\mathrm{HI}}$ level of $1 \times 10^{19} \mathrm{~cm}^{-2}$, which is on the same order as the analytical critical column densities derived by Bland-Hawthorn et al. (2017), Maloney (1993), and Dove \& Shull (1994) (i.e., where the Hi transitions from mostly neutral to mostly ionized) and is approximately the $5 \sigma N_{\mathrm{HI}}$ detection limit of the native resolution WSRT data over a $20 \mathrm{~km} \mathrm{~s}^{-1}$ line. Secondly, diffuse Hi is implied to be extended over angular scales larger than the maximum recoverable angular scale of the WSRT $\left(\sim 20^{\prime}\right)$. A lower $f_{19}$ value measured for a well-resolved source (relative to the larger GBT beam) would therefore demonstrate that most of the Hi resides at higher column densities, indicating no signs of an diffuse inflowing component that can be observed in emission. On the other hand, a higher $f_{19}$ value would suggest the WSRT resolves out large scale Hi features that possess $N_{\mathrm{HI}}$ levels lower than $\sim 10^{19} \mathrm{~cm}^{-2}$.

Because $N_{\mathrm{HI}}$ is directly proportional to the measured brightness temperature, which is itself averaged over the beam solid angle, the usefulness of the $f_{19}$ parameter to characterize the diffuse Hi mass fraction relies on the sources being well-resolved. Once the source becomes unresolved, our measurement - that is effectively the convolution of the true Hi emission distribution with the larger beam — will spread 
the flux of the out to angular scales where the beam response is lower, thus measuring low column density values that are no longer physical but still nevertheless begin to dominate the overall Hi mass fraction and bias our quantity. While $f_{19}$ provides a quantity that can be related to properties predicted to be influential to accretion, given that the sources presented in this work are at different distances, span a range of physical scales, and are in general inhomogeneous, acute attention to several resolution indicators, which will be discussed below, is required to ensure a $f_{19}$ value for a given galaxy is not biased by resolution effects.

The $f_{19}$ values are summarized in Table 2.3. In general, the $f_{19}$ values derived from the convolved WSRT data are similar to their regridded GBT counterparts. In the cases where the convolved WSRT values are higher, the increase can be attributed to the increased column density sensitivity when the WSRT data (where emission fills the synthesized beam) is convolved to low resolution. In addition to the four sources discussed above, we also compute $f_{19}$ for three galaxies which have similar GBT data: NGC2403 (also a HALOGAS source) (Fraternali et al. 2001, 2002; de Blok et al. 2014a), NGC2997 (Pisano, 2014), and NGC6946 (with additional deep WSRT data; Pisano 2014; Boomsma et al. 2008).

Our initial small sample size inhibits us from making any significant conclusions about the relations between fraction of diffuse Hi and any of the properties which trace simulation predictions. However, investigating the trends within our initial small sample will provide intuition on any underlying correlations that may be revealed as we build up our sample and flesh out issues due to resolution effects.

As mentioned above, it is critical to rule out observational or resolution bias as 
a source for potential trends. Figure 2.15a plots $f_{19}$ for each source as a function of projected Hi angular area computed from the number of pixels above the associated $2 \sigma N_{\mathrm{HI}}$ level from Table 2.2 in the unmasked high-resolution WSRT $N_{\mathrm{HI}}$ images. Note that in each panel the blue circles denote $f_{19}$ values derived from convolved WSRT data and the orange inverted triangles represent GBT $f_{19}$ values. The vertical dashed line represents the area of the GBT beam above which extended source structure becomes resolved. While it is encouraging to see all of the projected HI angular area data points fall to the right of the GBT beam area, this is only one indication that our $f_{19}$ parameter is not biased by resolution effects. Because $f_{19}$ is essentially the one minus the ratio of the integral of the cumulative HI mass distribution taken from bins larger than $\log _{10}\left(N_{\mathrm{HI}} / \mathrm{cm}^{2}\right)=19.0$ to an integral taken over the full range of $N_{\mathrm{HI}}$ bins, a better indicator for possible resolution bias is to ensure the data points of the cumulative Hi mass distribution for a given source does not trace the simulated unresolved observation (i.e., the GBT beam profile).

In the case of Figure 2.15a, the highest $f_{19}$ value is indeed associated with NGC4414, whose angular area is only a few times that of the GBT beam. Figure 2.10b shows the WRST data cumulative Hi mass fraction is consistent with the observation of an unresolved source within the large GBT beam at higher $N_{\mathrm{HI}}$ bins, while the GBT cumulative Hi mass distribution shows only marginal improvement over the simulated unresolved observation. The slight trend between increasing distance and increasing $f_{19}$ values in Figure $2.15 \mathrm{~b}$ does hint that $f_{19}$ will be influenced by the large GBT beam. The high $f_{19}$ values in both data sets for NGC4414 reveal how the effects of resolution can bias the calculation of $f_{19}$. That said, we also see 
sources with comparable $f_{19}$ values over a large range of projected angular areas whose cumulative Hi mass functions do not trace a simulated observation of an unresolved source. Figures $2.15 \mathrm{a}$ and $\mathrm{b}$ therefore demonstrate that $f_{19}$ is not generally biased when applied to well-resolved sources.

The physical extent of a diffuse gas reservoir should ideally scale with a diffuse HI mass fraction. Plotting $f_{19}$ as a function of physical projected HI area in Figure $2.15 \mathrm{c}$ shows that the highest $f_{19}$ value is associated with the second largest physical extent; however, the high $f_{19}$ value is very likely biased due to the greater distance of the source. On the other hand, the second highest $f_{19}$ value corresponds to the very well-resolved galaxy of NGC4565, demonstrating the expected scaling. That said, the fact this particular GBT $f_{19}$ value is the second highest value of the sample by only about a factor of two underscores the lack of a significant diffuse $\mathrm{HI}$ around NGC4565.

A relationship between $f_{19}$ and galaxy properties investigated in simulations (e.g., Kereš et al. 2005, 2009) should give some indication as to whether any newly probed diffuse component could be related to accretion from the IGM. For example, simulations show cold mode accretion is still an available channel for gas accretion around nearby galaxies with relatively low halo masses $\left(M_{\text {halo }} \leq 10^{11.4} M_{\odot}\right)$ and in low density environments. Any trend between excess Hi and properties known to be responsible for the presence of excess Hi could provide observational evidence, albeit indirect, that the observed excess HI accreted directly from the IGM. Figure 2.15d plots $f_{19}$ as a function of galaxy density, or the number of galaxies $\mathrm{Mpc}^{-3}(\rho)$; we estimate $\rho$ by using values derived in Tully (1988). Any correlation between $f_{19}$ and 
$\rho$ should provide insight into the influence of tidal forces. No obvious trend presents itself here. The absence of a trend in the context of the full sample would be indicative of the relative unimportance of tidal interactions as compared to outflows or accretion to the presence of extraplanar and/or an extended gas reservoir.

Figure 2.15e plots $f_{19}$ as a function of the baryonic mass $\left(M_{\text {bary }}\right)$ where the vertical dashed lines represent the threshold set by the simulations of Kereš et al. (2005) where cold mode accretion is predicted to be the dominant mechanism of gas accretion. Assuming the dust and molecular gas components to be negligible to the total gas mass, we calculate $M_{\text {bary }}$ to be equal to $M_{\mathrm{HI}} \cdot 1.36+M_{*}$, where $M_{\mathrm{HI}}$ is measured by the GBT. The factor of 1.36 in the leading term corrects for the fraction of neutral He. There is no apparent trend between $f_{19}$ and $M_{\text {bary }}$. Additionally, Figure $2.15 \mathrm{f}$ presents the relationships between $f_{19}$ and rotational velocity $\left(V_{\text {rot }}\right)$. The dashed line in this plot shows the observational threshold such that galaxies with $V_{\text {rot }} \leq 125 \mathrm{~km} \mathrm{~s}^{-1}$ are expected to be gas dominated due to continuous sustained growth (Kannappan, 2004; Kannappan et al., 2013). Again, no discernible trend is observed.

The final origin scenario for a large diffuse component in these galaxies is related to outflows due to star formation activity in the disk. The relationship between $f_{19}$ and specific star formation rate ( $\mathrm{sSFR}$ ), which is the star formation rate per unit stellar mass, is presented in Figure 2.15g; $f_{19}$ as a function of the surface density of star formation is shown in Figure 2.15h. We derive the surface density of star formation by dividing the SFR by the de-projected physical area. We compute the physical area assuming each galaxy face-on is roughly circular and utilize the 
de-projected and extinction corrected semi-major angular diameter computed from the B-band $25 \mathrm{mag} \operatorname{arcsec}^{-2}$ isophote (de Vaucouleurs et al., 1976). Panel h show subtle indications of increasing $f_{19}$ with increased star formation activity. A positive correlation suggests more energy per gravitational potential in such galaxies, which points to the possible existence of outflows.

Some, if not most, of the extraplanar gas observed around nearby galaxies must originate from outflows described by galactic fountain (Bregman 1980; Norman \& Ikeuchi 1989; Fraternali 2017), and are driven by the injection of momentum and energy into the local interstellar medium from supernovae. In most cases, such outflows are the more feasible origin for diffuse Hi in the form of extraplanar gas. Fraternali \& Binney (2006) and Fraternali \& Binney (2008) outline dynamical models that accurately reproduce the general vertical distribution of gas in NGC891 and NGC2403 with a necessary energy injection of only $<4 \%$ of the energy released by supernovae. But while the general distribution is reproduced, the rotational velocity of the model halos is much too high compared to observations. The discrepancy between modeled and observed rotational velocities suggested the acquisition of low-angular momentum gas from the IGM in order to slow the model halos. More recently, however, Marinacci et al. (2010) and Fraternali (2017) show that including the effects of condensation, drag (ram pressure), and radiative cooling in these ballistic models of fountain gas will prevent momentum loss in the cold gas while also increasing the transfer of momentum to intermediate temperature material as opposed to the hot coronal gas. The increase in rotational velocity is therefore mitigated, thus bringing theses models into agreement with observational data. By 
incorporating radiative cooling of the hot coronal gas, it can condense in the wake of fountain clouds which consequently raises the accretion rate of low-metallicity gas onto the central disk of an embedded galaxy. Specifically, Fraternali (2017) recover cold gas accretion rates comparable with the the SFR of NGC891 and NGC2403.

We end this section by posturing what type of observational constraints between $f_{19}$ and the inherent galaxy properties shown in Figure 2.15 would indicate the possibility of observing active cold mode accretion. The first necessity would be a $f_{19}$ value from the GBT data that is well above the WSRT and well to the right of the vertical dashed line in Figure 2.15a. This would indicate that the WSRT had resolved out significantly extended structure and physical column densities as the emission has filled the large GBT beam. That same large $f_{19}$ GBT data point would then need to be associated with a small $\rho$ to indicate isolation and that tidal interactions are not likely to be the origin of the diffuse environment. We would next need to see the $f_{19}$ value fall below the $M_{\text {bary }}$ and $V_{\text {rot }}$ thresholds to ensure the galaxy is consistent with the predictions of the cosmological simulations. Finally, the presence of a significant diffuse Hi component would need to be associated with a relatively insignificant amount of star formation activity ruling out the presence of substantial outflows and subsequent condensation from the fountain activity.

The derived properties of these four sources are summarized in Table 2.3. The columns are (1) Source Name; (2) Hi mass measured in the GBT data; (3) HI mass measured in the convolved WSRT data; (4) HI mass as measured in the high-resolution WSRT data; (5) stellar mass derived from the WISE All Sky Image Atlas; (6) baryonic mass; (7) deprojected physical area; (8) rotation velocity from 


\begin{tabular}{|c|c|c|c|c|c|c|c|}
\hline Derived Properties & NGC891 & NGC925 & NGC2403 & NGC2997 & NGC4414 & NGC4565 & NGC6946 \\
\hline GBT Hi Mass $\left[10^{9} M_{\odot}\right]$ & $3.86 \pm 0.19$ & $5.79 \pm 0.29$ & $3.39 \pm 0.37$ & $7.0 \pm 1.0$ & $5.43 \pm 0.27$ & $7.33 \pm 0.37$ & $3.80 \pm 0.69$ \\
\hline Conv. WSRT Hi Mass $\left[10^{9} M_{\odot}\right]$ & $3.81 \pm 0.19$ & $5.54 \pm 0.28$ & - & - & $4.56 \pm 0.22$ & $7.46 \pm 0.39$ & - \\
\hline High-Res. WSRT Hi Mass $\left[10^{9} M_{\odot}\right]$ & $3.90 \pm 0.18$ & $5.57 \pm 0.28$ & - & - & $4.63 \pm 0.23$ & $7.55 \pm 0.38$ & - \\
\hline $\mathrm{M}_{*}\left[10^{10} M_{\odot}\right]$ & $0.30 \pm 0.10$ & $4.40 \pm 0.10$ & $0.39 \pm 0.01$ & $8.70 \pm 0.10$ & $4.15 \pm 0.10$ & $6.15 \pm 0.10$ & $0.12 \pm 0.01$ \\
\hline $\mathrm{M}_{\text {bary }}\left[10^{10} M_{\odot}\right]$ & $0.82 \pm 0.02$ & $5.20 \pm 0.10$ & $0.86 \pm 0.03$ & $9.71 \pm 0.18$ & $4.89 \pm 0.10$ & $7.15 \pm 0.10$ & $6.29 \pm 0.59$ \\
\hline Deprojected Physical Area $\left[\mathrm{kpc}^{2}\right]$ & $603 \pm 6$ & $490 \pm 6$ & $122 \pm 3$ & $726 \pm 11$ & $241 \pm 24$ & $976 \pm 10$ & $416 \pm 3$ \\
\hline $\mathrm{V}_{\text {rot }}^{\mathrm{a}}\left[\mathrm{km} \mathrm{s}^{-1}\right]$ & $212 \pm 2$ & $105 \pm 2$ & $196 \pm 1$ & $145 \pm 3$ & $218 \pm 6$ & 244 & $315 \pm 12$ \\
\hline WSRT $f_{19}$ & $0.011 \pm 0.001$ & $0.014 \pm 0.001$ & - & - & $0.064 \pm 0.004$ & $0.006 \pm 0.001$ & $0.004 \pm 0.001$ \\
\hline GBT $f_{19}$ & $0.005 \pm 0.001$ & $0.008 \pm 0.001$ & $0.005 \pm 0.001$ & $0.003 \pm 0.001$ & $0.046 \pm 0.002$ & $0.008 \pm 0.001$ & $0.003 \pm 0.001$ \\
\hline
\end{tabular}

Table 2.3 Summary of Derived Properties

\footnotetext{
${ }^{1}$ Rotation velocity taken from HYPERLEDA search
}

HYPERLEDA (9) $f_{19}$ measured in the convoled WSRT cube; and (10) $f_{19}$ measured in the GBT cube. The relationships in this section should be considered diagnostic in that we are trying to develop intuition into what trends should exist in galaxies that are actively undergoing cold mode accretion. The low number sources studied in this work inhibit any conclusions. We will continue to build our statistical sample with future analysis of the HALOGAS, THINGS, and ultimately the MeerKAT HI Observations of Nearby Galactic Objects: Observing Southern Emitters (MHONGOOSE; de Blok et al. 2017) and the Imaging Galaxies Inter-galactic and Nearby Environment (IMAGINE) galaxy samples in order to search for correlations between the presence of a significant diffuse Hi environment and the intrinsic galaxy properties which should trace accretion from the IGM in the local Universe.

\subsection{Conclusions and Future Work}

We presented initial analysis of deep $\left(N_{\mathrm{HI}} \sim 10^{18} \mathrm{~cm}^{-2}\right)$ GBT observations of four sources (NGC891, NGC925, NGC4414, and NGC4565) out of 24 total sources 
in the HALOGAS survey. These observations are among the most sensitive Hi observations of external galaxies to date. In order to directly compare interferometer and single dish data, we solve for an optimal smoothing kernel specific to each source and convolve the WST data to GBT angular resolution. Our main conclusions are:

- As we do not find significant spatially extended Hi features, we conclude that the WSRT data do an excellent job recovering the diffuse $\left(18 \leq \log _{10}\left(N_{\mathrm{HI}}\right) \leq\right.$ 19) HI around these four sources. In the case of NGC925, we detect about $20 \%$ more Hi than observations done with the VLA as part of the THINGS survey. The discrepancy is likely due in large part to the increased surface brightness sensitivity of the WSRT data since the ability to detect extended structure between the two surveys is very similar. The excellent agreement between the global Hi profiles, cumulative Hi mass as a function of $N_{\mathrm{HI}}$, radial mean column density profiles, and radial cumulative flux for the GBT and convolved WSRT data provides additional evidence in support of this conclusion.

- The cumulative Hi mass as a function of Hi column density reveals the diffuse Hi associated with these galaxies does not change significantly over the range $\log _{1} 0\left(N_{\mathrm{HI}} / \mathrm{cm}^{2}\right)=18.0$ to $\log _{10}\left(N_{\mathrm{HI}} / \mathrm{cm}^{2}\right)=19.5$. The flat behavior is consistent with predictions from simulations, which show the neutral fraction is around $1 \%$ at $\log _{1} 0\left(N_{\mathrm{HI}} / \mathrm{cm}^{2}\right)=18.0$. Scaling our GBT beam model to the peak column density of the GBT data and repeating our analysis to essentially simulate an unresolved source produces a similarly flat distribution, which suggests the lowest column density bins include some values that trace 
the extended structure of the GBT beam. That said, there is generally a moderate offset between the data and model cumulative HI mass distributions. The overall agreement between the GBT and WSRT data sets, if extended to the other sources in our survey, suggests future surveys must probe column densities at the $\sim 10^{17} \mathrm{~cm}^{-2}$ level to increase the probability of detecting HI associated with cosmic web structure or possibly cold-mode accretion.

- We define a parameter, $f_{19}$, equal to one minus the ratio between the HI mass measured at and above $\log _{10}\left(N_{\mathrm{HI}}\right)=19$ and the source's total Hi mass. We find that, on average (and excluding data that may suffer from resolution effects), this value is equal to $2 \%$, indicating the diffuse extended disks of these galaxies do not constitute a significant fraction of the overall mass.

One observational method to differentiate between inflow/outflow origins is a measure of metallicity using UV absorption lines (e.g., S II) utilizing the Cosmic Origins Spectrograph on the Hubble Space Telescope. If a significant diffuse Hi feature is seen around a source as we analyze the full survey, and a fortuitous background quasar along the line of sight, a metallicity of $\sim 0.1 Z_{\odot}$ would be highly indicative of a CGM origin.

To establish or rule out cold mode accretion as a feasible avenue for nearby galaxies to refuel their gas content, we must continue to analyze galaxies within the HALOGAS sample that satisfy the mass constraints set by simulations, show large diffuse Hi mass fractions and low SFRs, and reside in low density environments. Due to our small sample size we can only present foundational work to uncover 
any underlying correlations between large mass fractions of diffuse Hi and galaxy properties. Future work will focus on the analysis techniques presented here in order investigate the origins of these large Hi filaments, apply short spacing corrections to the WSRT data, and continue the investigation into role of $\mathrm{HI}$ in galaxy evolution. 


\title{
Chapter 3
}

\section{A GBT Survey of the HALOGAS Galaxies and Their Environments II: The Full Survey ${ }^{1}$}

\begin{abstract}
The analysis introduced and applied to the four HALOGAS sources of the pilot survey in the previous chapter is extended to the other sources in the sample. While two sources, NGC4258 and NGC4631 show evidence for large-scale diffuse $\mathrm{HI}$, the origin of these features are almost certainty due to tidal interactions with nearby companions. There is no other evidence that links the gas in the disk to the potential accreting gas from the IGM in the well-resolved galaxies of the sample. The dearth of diffuse HI at $N_{\mathrm{HI}} \gtrsim 10^{18}$ $\mathrm{cm}^{-2}$ around the HALOGAS galaxies further advocates for the notion that Hi surveys planned for future facilities need to invest the integration time required to probe down to $N_{\mathrm{HI}} \sim 10^{17} \mathrm{~cm}^{-2}$.
\end{abstract}

\subsection{Introduction}

Chapter 2 introduced the motivation of the search for diffuse Hi around nearby galaxies. Namely, galaxies must somehow be replenishing their gas through accretion - whether from gas from the intergalactic medium (IGM) that has fallen through

\footnotetext{
${ }^{1}$ I wish to again acknowledge D. J. Pisano, G. Heald, T. H. Jarrett, W. J. G. de Blok, G. I. G. Józsa, E. Jütte, R. J. Rand, T. Oosterloo, and B. Winkel as collaborators on this work presented in this chapter.
} 
the halo, or through interactions between the hot halo gas and disk material (e.g. Kereš et al. 2005; Fraternali 2017). In either case, the neutral infalling gas will be at $N_{\mathrm{HI}}$ levels that have rarely been probed in emission, which would help to constrain the full spatial distribution of low column density gas around nearby galaxies. Analysis of low column density $N_{\mathrm{HI}}$ levels (i.e., those below $10^{19} \mathrm{~cm}^{-2}$ ) at the most extreme angular extents of galaxies' HI emission will characterize prevalent properties of the interface between the circumgalactic medium (CGM) and the edge of galaxies where gas accretion is to be taking place.

Several low column density filamentary features with $N_{\mathrm{HI}} \sim 10^{18} \mathrm{~cm}^{-2}$ have been observed in galaxies with known extraplanar components such as NGC2403 (Fraternali et al., 2002; de Blok et al., 2014a) and NGC6946 (Boomsma et al., 2008; Pisano, 2014) that make up over $10 \%$ of the total HI mass while existing at $N_{\mathrm{HI}} \sim 10^{19} \mathrm{~cm}^{-2}$. Are such features common in nearby galaxies? Given that the recent photoionization models of Bland-Hawthorn et al. (2017) have shown gas at the $N_{\mathrm{HI}}$ level of $10^{18} \mathrm{~cm}^{-2}$ can reasonably survive even in the presence of an intergalactic radiation field (albeit with a high clumping factor), an extended Hi reservoir could feasibly be the natural extension of the observed extraplanar gas. The resolution of the GBT data prohibits highly detailed imaging of potential features between the $N_{\mathrm{HI}}$ levels of $10^{19} \mathrm{~cm}^{-2}$ to $10^{18} \mathrm{~cm}^{-2}$. However, unlike the interferometer data that has missing data at the lowest spatial frequencies, the GBT data possesses information on the true total flux and therefore total Hi mass. Utilizing only wellresolved sources, the analysis presented in Chapter 2 such as the cumulative Hi mass as a function of $N_{\mathrm{HI}}$ will be used to explicitly measure the mass fraction of the 
HI that remains sufficiently self-shielded to exist at $N_{\mathrm{HI}} \lesssim 10^{19} \mathrm{~cm}^{-2}$. Additionally, the radial profiles of mean $N_{\mathrm{HI}}$ and cumulative flux can be used to infer the the variations in the $N_{\mathrm{HI}}$ distributions at large angular extents.

While the next generation of radio telescopes are likely necessary to truly probe the emission from such low column density gas with less constraint on telescope time, the GBT data presented in this work can be utilized in many different ways to investigate how disk star formation is refueled by the IGM. In this chapter, the specific investigation focuses on the extension of the analysis from Chapter 2 to the entire GBT HALOGAS sample and offers a discussion on results in the context of current and future Hi surveys designed to detect low column density Hi in external galaxies. Given that only GBT data are available for analysis, the GBT data for the full sample and modifications to the analysis steps are discussed in Section 3.2; Section 3.3 briefly summarizes the known Hi properties of each galaxy; Section 3.4 presents the results; Section 3.5 discusses the implications for future Hi surveys given the analysis on the full GBT sample; and Section 3.6 presents the conclusions of the analysis applied to the full sample.

\subsection{Alterations to Analysis Methods}

The data to be analyzed in this chapter were obtained over the GBT projects GBT09B_034, GBT13B_406, GBT14A_511, GBT14B_293, and GBT16B_392. The observational configuration and data reduction was identical to what is described in Section 2.3.1. The additional project, GBT16B_392, was undertaken in September 
of 2016 to extend the map of NGC4258 by $2 \mathrm{deg}$ in $\alpha_{J 2000}$ and $1 \mathrm{deg}$ in $\delta_{J 2000}$ to the south in pursuance of completely mapping the initially observed companions. The data from GBT09B_034 and GBT11A_055 were obtained during very similar GBT observations (the major difference being that the backend was the old Spectrometer) for the THINGS galaxies, NGC2403 (de Blok et al., 2014a), NGC3198, and NGC5505 (Pisano; private communication) that are also sources in the HALOGAS survey. While $f_{19}$ parameters are derived for these sources, they are not included in the discussions of Section 3.3

In addition to adopting the same gain value of $1.86 \mathrm{~K} \mathrm{Jy}^{-1}$ (based on the aperture efficiency of 0.65 Boothroyd et al. (2011), the adopted flux uncertainty remains at an upper limit of $5 \%$ to encompass potential errors introduced by inaccurate band calibration, RFI, etc. Calibration and imaging of the data occurred in the same manner with the slight modification of smoothing the data before flux calibration to mitigate spurious spikes caused by internally generated RFI (likely caused by bad IF throughput) getting amplified when the reference spectrum is subtracted off during calibration.

Several of the HALOGAS sources are unresolved in the GBT beam. The unresolved nature of these sources severely complicates the computation of $f_{19}$ (see Equation 2.9, since no physically meaningful measurement of $N_{\mathrm{HI}}$ can be made. For this reason, NGC949, NGC4062, and UGC2082 are excluded from the analysis presented in this chapter. Two other sources, NGC4448 and NGC4274, are nondetections and are also omitted from the subsequent discussion. The global flux as a function of velocity for these sources are presented in Figures 3.1-3.5. 


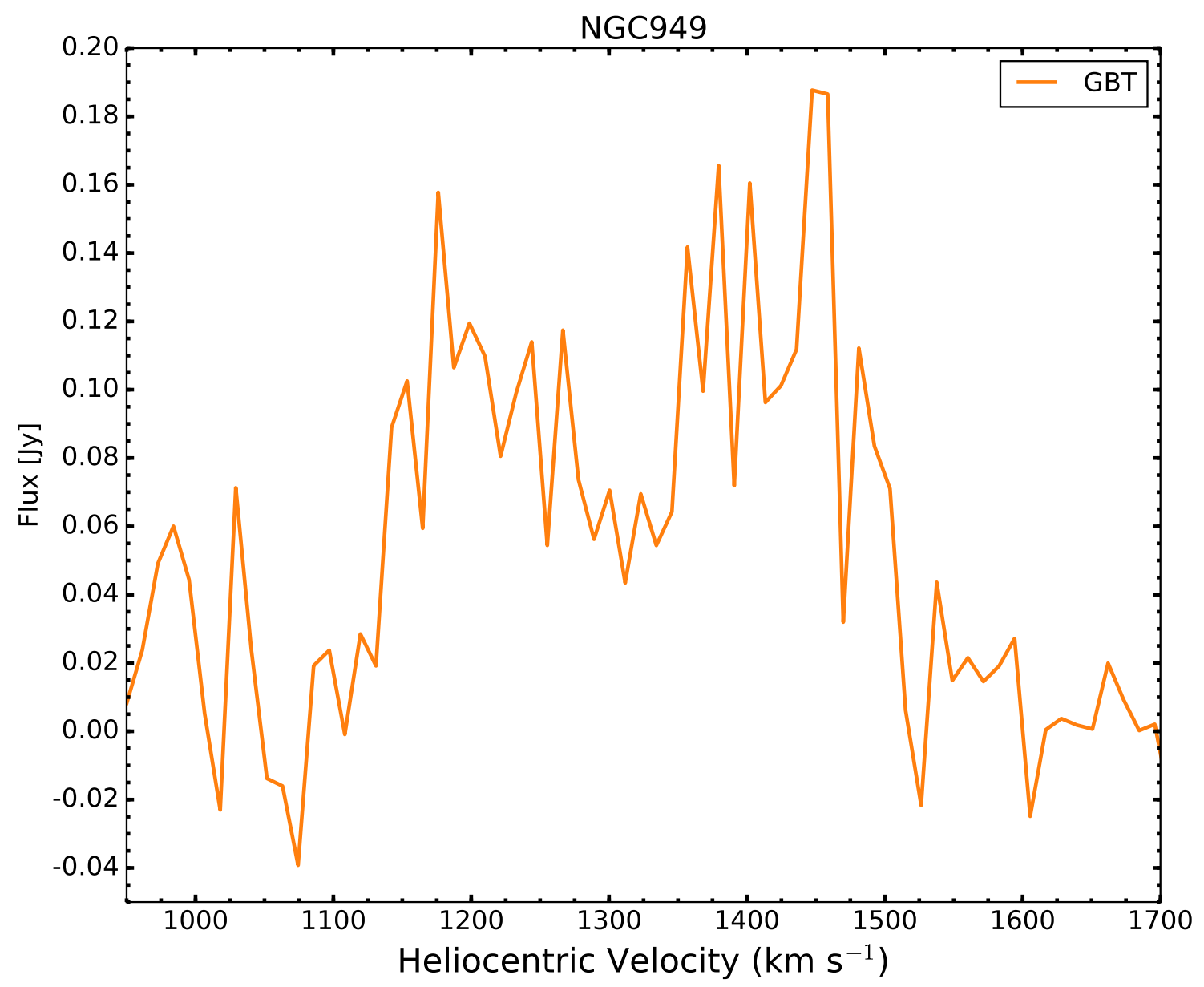

Figure 3.1 The global Hi profile for the unresolved source, NGC949. 


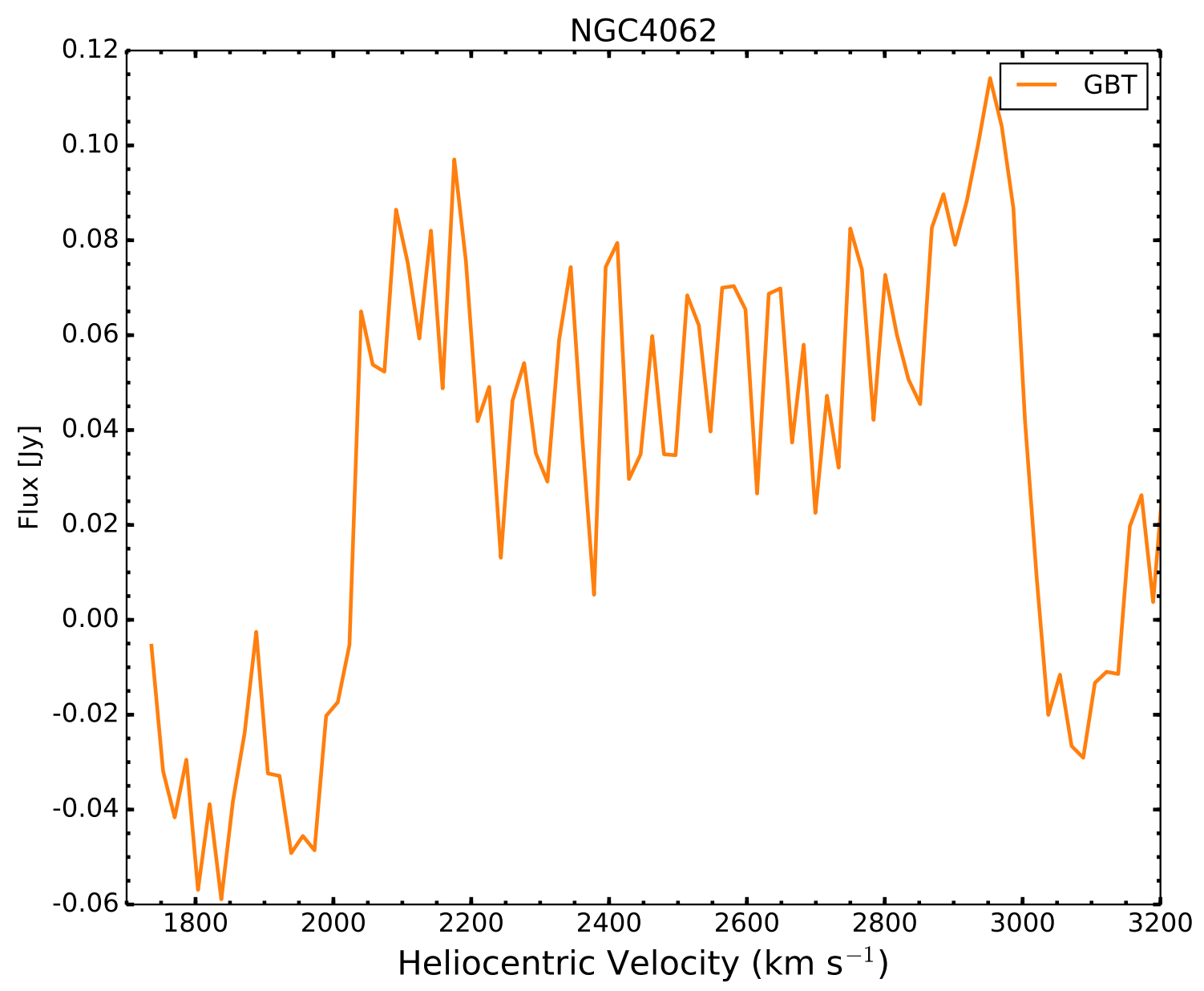

Figure 3.2 The global Hi profile for the unresolved source, NGC4062. 


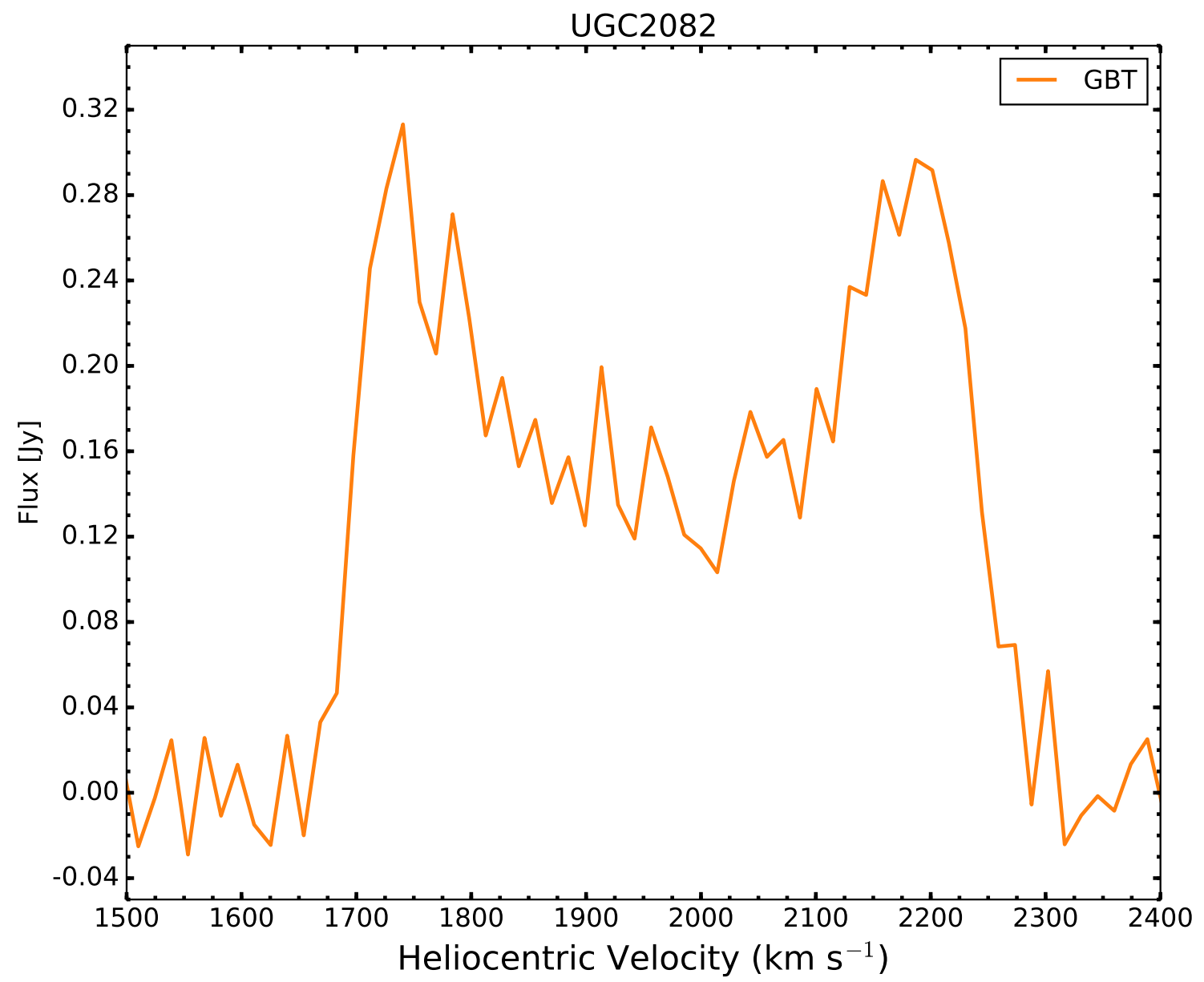

Figure 3.3 Global Hi profile for the unresolved source, UGC2082. 


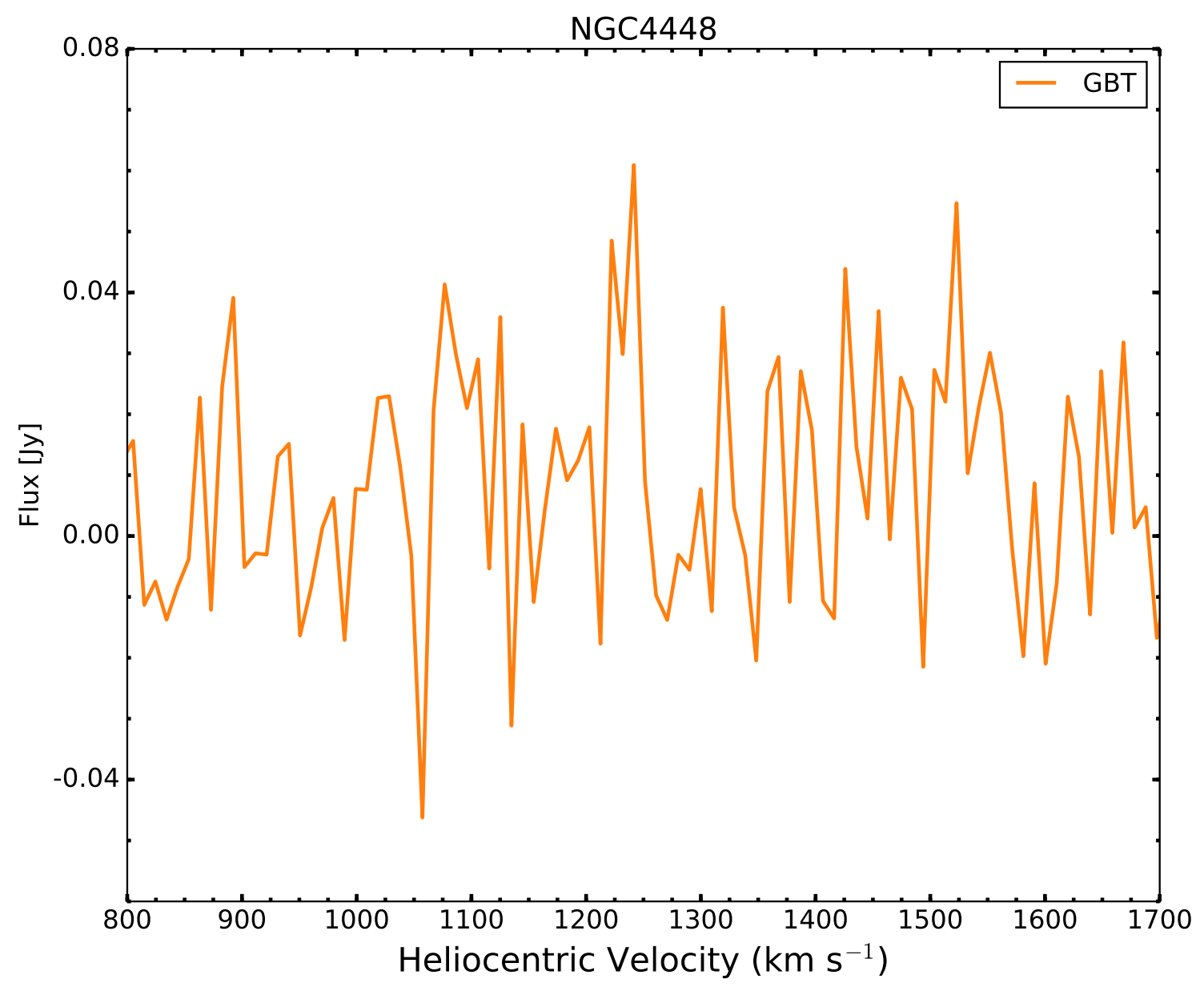

Figure 3.4 Global Hi profile for the non-detection of NGC4448. 


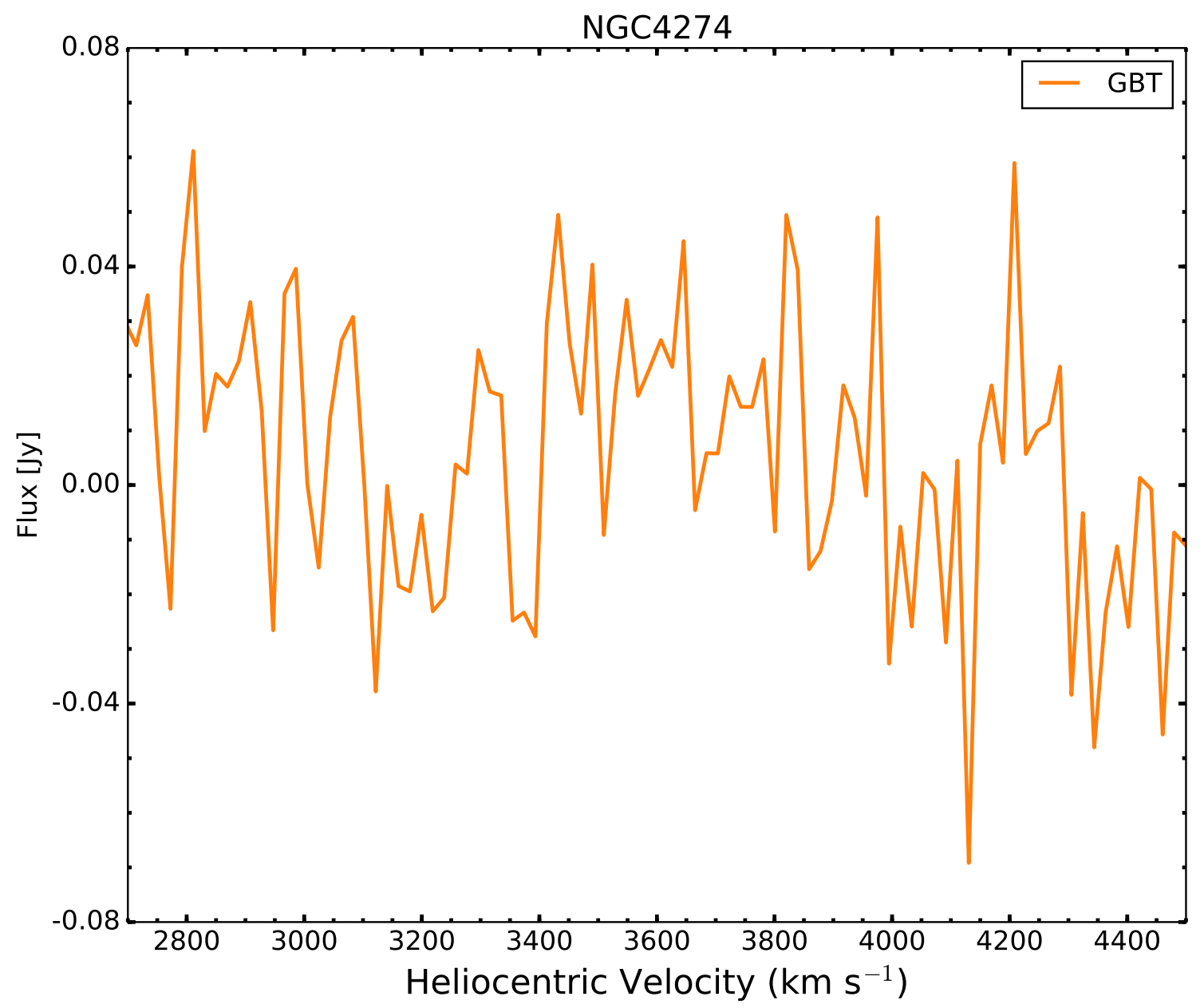

Figure 3.5 Global Hi profile for the non-detection of NGC4274. 
As in Chapter 2, the low column density Hi is characterized by a variety of metrics by constructing integrated Hi maps as in Section 2.4.1.2, measuring the total flux, the cumulative Hi mass (see Section 2.4.1.3, and the radial distributions of cumulative flux and the mean $N_{\mathrm{HI}}$. Note that, just as in Chapter 2, the GBT data are regridded to the standard pixel size of the HALOGAS data or $4 \times 4$ square arcseconds to ensure adequate sampling within a given bin of one-half the GBT beam. In addition to the image properties, the relationship between fraction of $\mathrm{HI}$ mass below a level of $N_{\mathrm{HI}}, f_{19}$, is compared to environmental properties predicted to be associated with cold mode accretion is fully explored. The general properties of the remaining sources in the sample are first briefly summarized before discussing overall results. Basic properties of the sources included in the subsequent analysis are listed in Table 3.1.

\subsection{The Full GBT Survey Data and Sample}

The following section briefly summarizes the properties of each source's data cube in the remaining sample.

\subsubsection{NGC672}

At the resolution of the GBT, the separation between NGC672 and its interacting companion, IC1727, falls within one GBT beam width such that the emission looks like a coherent structure (see Figure 3.6). Indeed, high resolution Hi and optical data have revealed that tidal forces have created a bridge of gas and stars 


\begin{tabular}{|c|c|c|c|c|c|c|c|c|}
\hline Source $^{\mathrm{a}}$ & $\alpha^{\mathrm{b}}$ & $\delta^{\mathrm{c}}$ & Systemic Velocity $\left[\mathrm{km} \mathrm{s}^{-1}\right]$ & $\sigma^{\mathrm{e}}[\mathrm{mK}]$ & $\sigma_{N H I}{ }^{\mathrm{f}}\left[\mathrm{cm}^{-2}\right]$ & $S^{\mathrm{g}}\left[\mathrm{Jy} \mathrm{km} \mathrm{s}{ }^{-1}\right]$ & $d_{\text {best }} \mathrm{h}[\mathrm{Mpc}]$ & $\mathrm{SFR}_{t o t}{ }^{\mathrm{i}}\left[M_{\odot} \mathrm{yr}^{-1}\right.$ \\
\hline NGC672 & $01^{h} 47^{m} 54.5^{s}$ & $27^{\circ} 25^{\prime} 58^{\prime \prime}$ & 429 & 12 & $2.2 \times 10^{17}$ & $253 \pm 13$ & 7.6 & $0.13 \pm 0.04$ \\
\hline NGC949 & $02^{h} 30^{m} 48.6^{s}$ & $37^{\circ} 08^{\prime} 12^{\prime \prime}$ & 609 & 13 & $2.4 \times 10^{17}$ & $19 \pm 1$ & 11.3 & $0.31 \pm 0.05$ \\
\hline UGC2082 & $02^{h} 36^{m} 16.1^{s}$ & $25^{\circ} 25^{\prime} 26^{\prime \prime}$ & 696 & 13 & $2.4 \times 10^{17}$ & $40 \pm 2$ & 14.4 & $0.04 \pm 0.01$ \\
\hline NGC1003 & $02^{h} 39^{m} 16.9^{s}$ & $40^{\circ} 52^{\prime} 20^{\prime \prime}$ & 626 & 12 & $2.2 \times 10^{17}$ & $181 \pm 9$ & 11.6 & $0.18 \pm 0.04$ \\
\hline UGC4278 & $08^{h} 13^{m} 14.6^{s}$ & $45^{\circ} 59^{\prime} 23^{\prime \prime}$ & 540 & 12 & $2.2 \times 10^{17}$ & $22 \pm 1$ & 13.6 & $0.18 \pm 0.02$ \\
\hline NGC2541 & $08^{h} 14^{m} 40.1^{s}$ & $49^{\circ} 03^{\prime} 42^{\prime \prime}$ & 548 & 12 & $2.2 \times 10^{17}$ & $265 \pm 13$ & 12 & $0.35 \pm 0.04$ \\
\hline NGC4062 & $12^{h} 04^{m} 03.8^{s}$ & $31^{\circ} 53^{\prime} 45^{\prime \prime}$ & 758 & 13 & $2.4 \times 10^{17}$ & $44 \pm 2$ & 16.9 & $0.67 \pm 0.07$ \\
\hline NGC4244 & $12^{h} 17^{m} 29.6^{s}$ & $37^{\circ} 48^{\prime} 26^{\prime \prime}$ & 244 & 13 & $2.4 \times 10^{17}$ & $380 \pm 19$ & 4.4 & $0.12 \pm 0.01$ \\
\hline NGC4258 & $12^{h} 18^{m} 57.5^{s}$ & $47^{\circ} 18^{\prime} 14^{\prime \prime}$ & 448 & 11 & $2.0 \times 10^{17}$ & $456 \pm 23$ & 7.6 & $0.71 \pm 0.09$ \\
\hline NGC4274 & $12^{h} 19^{m} 50.6^{s}$ & $29^{\circ} 36^{\prime} 52^{\prime \prime}$ & 930 & 10 & $1.9 \times 10^{17}$ & - & 19.4 & $1.2 \pm 0.1$ \\
\hline NGC4448 & $12^{h} 28^{m} 15.4^{s}$ & $28^{\circ} 37^{\prime} 13^{\prime \prime}$ & 661 & 7 & $1.3 \times 10^{17}$ & - & 9.7 & $0.06 \pm 0.01$ \\
\hline NGC4559 & $12^{h} 35^{m} 57.6^{s}$ & $27^{\circ} 57^{\prime} 36^{\prime \prime}$ & 807 & 11 & $2.0 \times 10^{17}$ & $272 \pm 14$ & 7.9 & $0.69 \pm 0.07$ \\
\hline UGC7774 & $12^{h} 36^{m} 22.7^{s}$ & $40^{\circ} 00^{\prime} 19^{\prime \prime}$ & 526 & 11 & $2.0 \times 10^{17}$ & $28 \pm 1$ & 24.4 & $0.10 \pm 0.01$ \\
\hline NGC4631 & $12^{h} 42^{m} 08.0^{s}$ & $32^{\circ} 32^{\prime} 29^{\prime \prime}$ & 606 & 12 & $2.2 \times 10^{17}$ & $1048 \pm 52$ & 7.6 & $1.17 \pm 0.15$ \\
\hline NGC5023 & $13^{h} 12^{m} 12.6^{s}$ & $44^{\circ} 02^{\prime} 28^{\prime \prime}$ & 807 & 12 & $2.2 \times 10^{17}$ & $58 \pm 3$ & 6.6 & $0.02 \pm 0.03$ \\
\hline NGC5229 & $13^{h} 34^{m} 02.8^{s}$ & $47^{\circ} 54^{\prime} 56^{\prime \prime}$ & 407 & 11 & $2.0 \times 10^{17}$ & $22 \pm 1$ & 5.1 & $0.009 \pm 0.001$ \\
\hline NGC5585 & $14^{h} 19^{m} 48.2^{s}$ & $56^{\circ} 43^{\prime} 45^{\prime \prime}$ & 293 & 12 & $2.2 \times 10^{17}$ & $115 \pm 6$ & 8.7 & $0.41 \pm 0.04$ \\
\hline
\end{tabular}

Table 3.1 Summary of Observations and Properties

${ }^{a}$ Source name

${ }^{b}$ Right Ascension (J2000)

${ }^{c}$ Declination (J2000)

${ }^{d}$ Systemic Velocity

${ }^{e}$ rms noise per $5.2 \mathrm{~km} \mathrm{~s}^{-1}$ velocity channel

${ }^{f} 1 \sigma N_{\mathrm{HI}}$ uncertainty over a $20 \mathrm{~km} \mathrm{~s}^{-1}$ line

${ }^{g}$ Total flux

${ }^{h}$ Adopted distance from H11

${ }^{i}$ SFRs derived from WISE; values for NGC5023 taken from (Heald et al., 2012) 


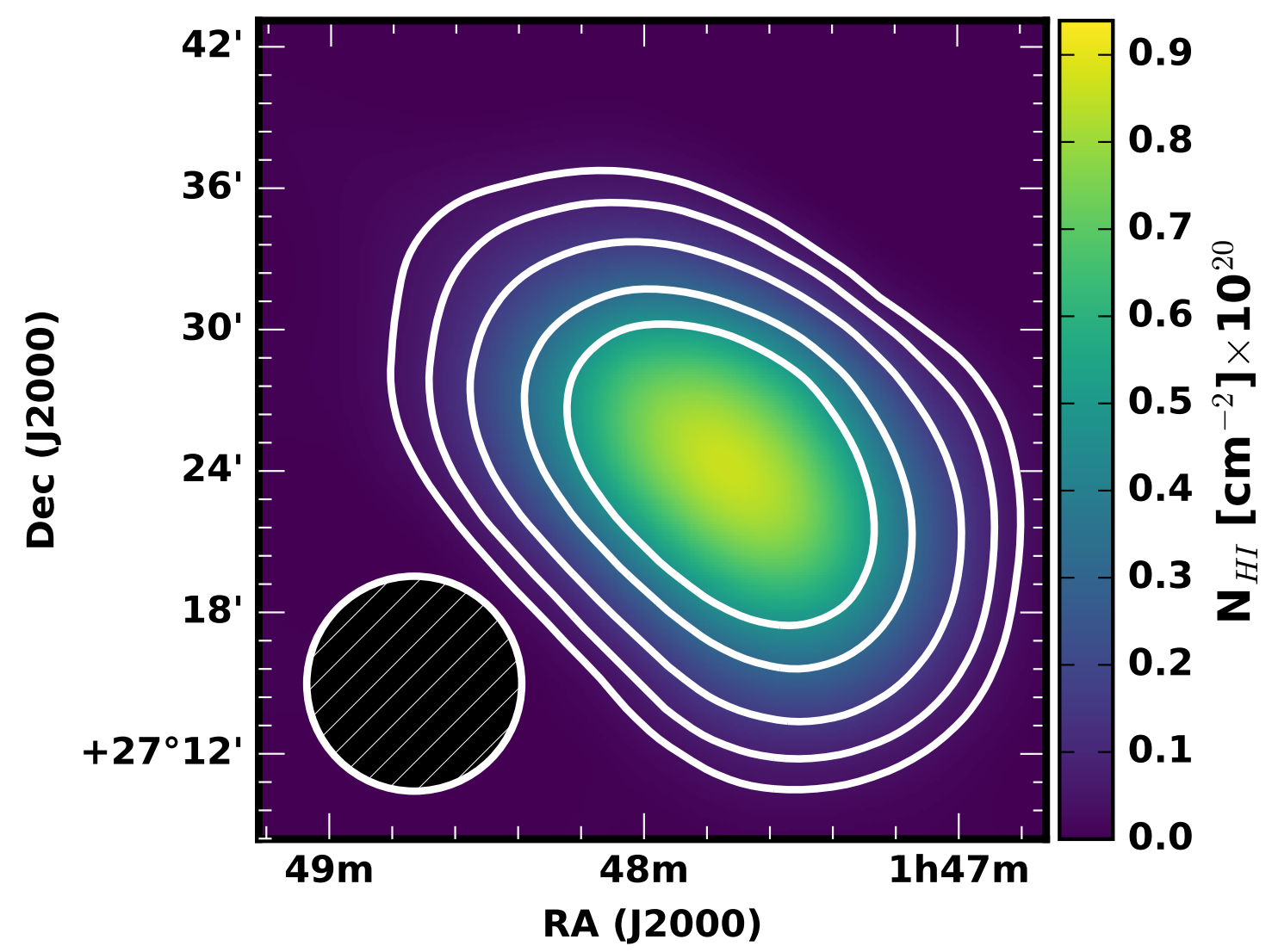

Figure 3.6 $N_{\mathrm{HI}}$ map of NGC672. The white contours begin at $4 \times 10^{18} \mathrm{~cm}^{-2}$ and continues at $2,4,8$, and 12 times that level.

connecting these two galaxies (Combes et al., 1980; Sohn \& Davidge, 1996). Another companion, AGC111945, sits outside of the field of view to the west, has no apparent connection to the interacting system of NGC672 and IC1727, and has a total flux density of $(3.08 \pm 0.15) \mathrm{Jy} \mathrm{km} \mathrm{s}^{-1}$, which is consistent with the value reported from the ALFALFA Survey (Haynes et al., 2011).

\subsubsection{NGC1003}

The nearly edge-on galaxy, NGC1003, shown in Figure 3.7 displays a reasonably thick disk with a scale height of $1 \mathrm{kpc}$, and, is radially extended such that the 


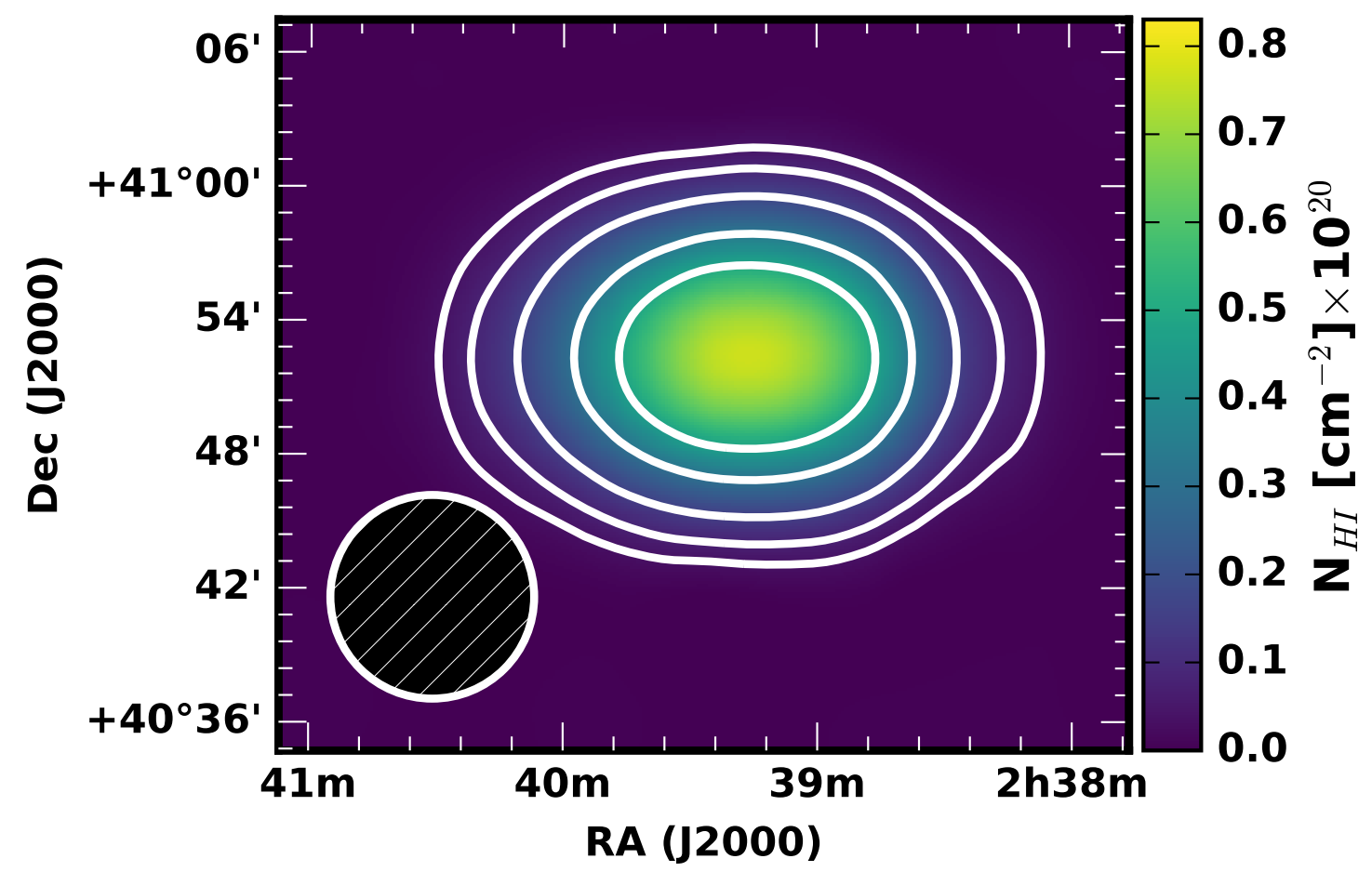

Figure $3.7 N_{\mathrm{HI}}$ map of NGC1003. The contours are at the same levels quoted in Figure 3.6.

Hi stretches out to five times the optical disk (Rand et al., 2011), typical for HI in spiral galaxies. Through tilted-ring modeling of the high-resolution WSRT data, that same study reveals a significant warp. Additionally, two previously undetected discrete clouds within the halo are observed that are likely high and intermediate velocity cloud analogues. There are no obvious signs of low column density features that would be resolved by the GBT. A nearby source, UGC2034, is also detected in the GBT map, but it's proximity to the edge of the field is not conducive to a reliable flux density measurement. 


\subsubsection{UGC4278}

UGC4278 is another edge-on galaxy within the HALOGAS sample. It has a very nearby ( $\sim 10$ arcminutes) companion, IC2233, to the southeast. That said, the galaxies do not appear at all to be interacting — even with similar systemic velocities. The shared contours in Figure 3.8 between the two galaxies, while appearing as a common Hi envelope, are due to the overlapping Hi distributions of the two disks within the large GBT beam. Other evidence that these galaxies are not interacting is the lack of an asymmetric Hi distribution in the high resolution observations data presented by Stil \& Israel (2002). UGC4278 is small with very little star formation activity. Preliminary Hi modeling of this source by Serra et al., (in prep) shows no obvious warps nor vertical lags in the gas rotation.

\subsubsection{NGC2541}

The moderately inclined SABc galaxy, NGC2541 (see Figure 3.9), while a member of the NGC2841 galaxy group, does not have any nearby companions that sit completely within the field of view mapped by the GBT; NGC2552 is the only observed companion that sits at the edge of the map to the northeast. Preliminary analysis of the high-resolution HALOGAS data do not reveal additional and distinct Hi clouds in the regions that extend into the halo of this source (E. Jütte 2018; in preparation). The integrated Hi map derived from the GBT data in Figure 3.9 show that this source is well resolved enough to be a good candidate to search for previously unobserved low column density Hı. 


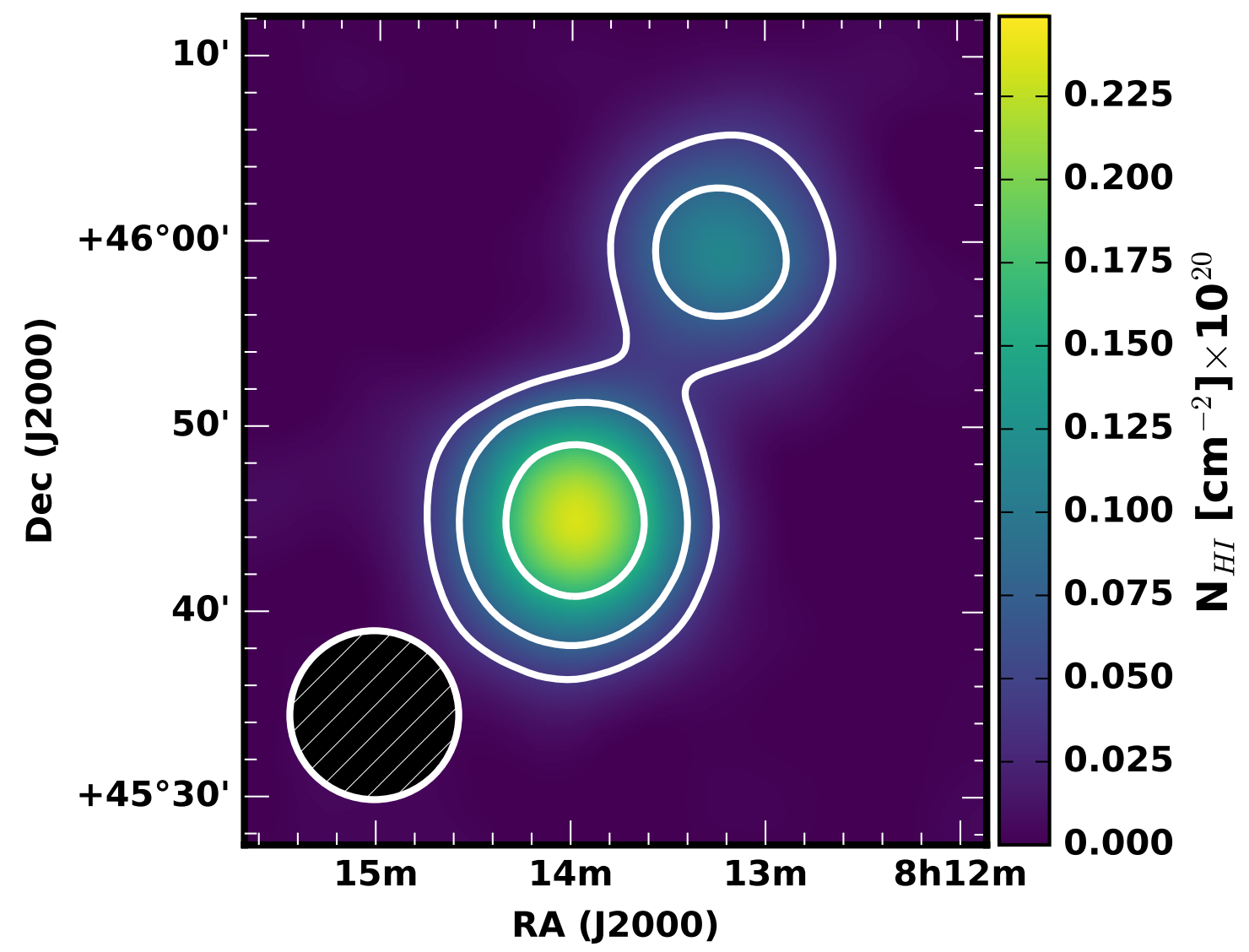

Figure $3.8 N_{\mathrm{HI}}$ map of UGC4278. The contours are at the same levels quoted in Figure 3.6. 


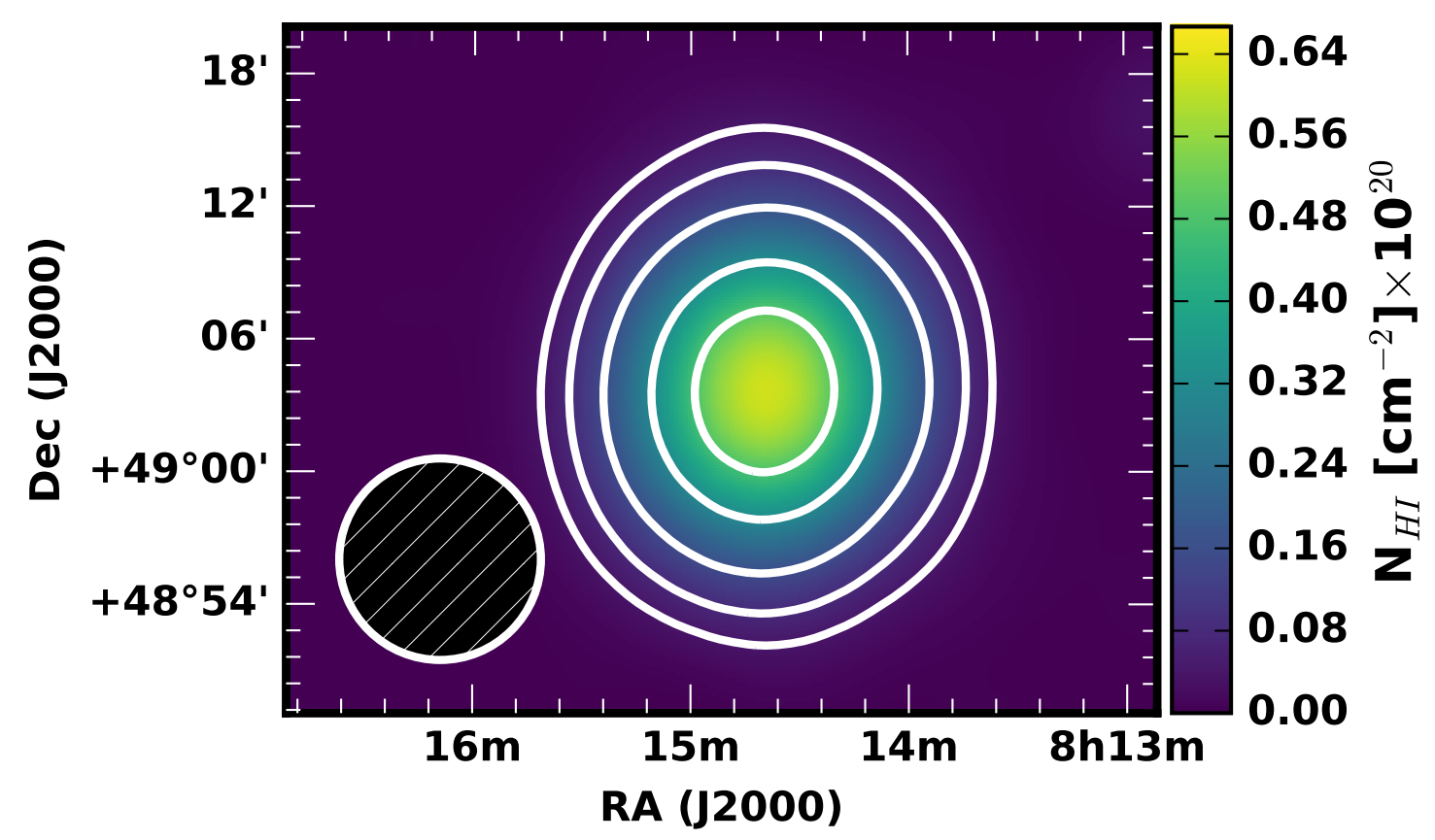

Figure $3.9 N_{\mathrm{HI}}$ map of NGC2541. The contours are at the same levels quoted in Figure 3.6.

\subsubsection{NGC4244}

NGC4244 is yet another highly inclined $\left(i \sim 85^{\circ}\right)$ galaxy whose high-resolution HALOGAS data has been fit to tilted ring models of the Hi kinematics and distribution in search for an extraplanar gas component as well as a rotation lag. Zschaechner et al. (2011). Unlike similar edge-on galaxies such as NGC891, the kinematic models show evidence for a warped disk both along and perpendicular to the line of sight but no extended Hi envelope. Nevertheless, a vertical velocity gradient is detected that decreases radially.

Similar to the high-resolution data, the $N_{\mathrm{HI}}$ map in Figure 3.10 and inspection of the cube do not reveal any potential diffuse Hi features or nearby companions. 


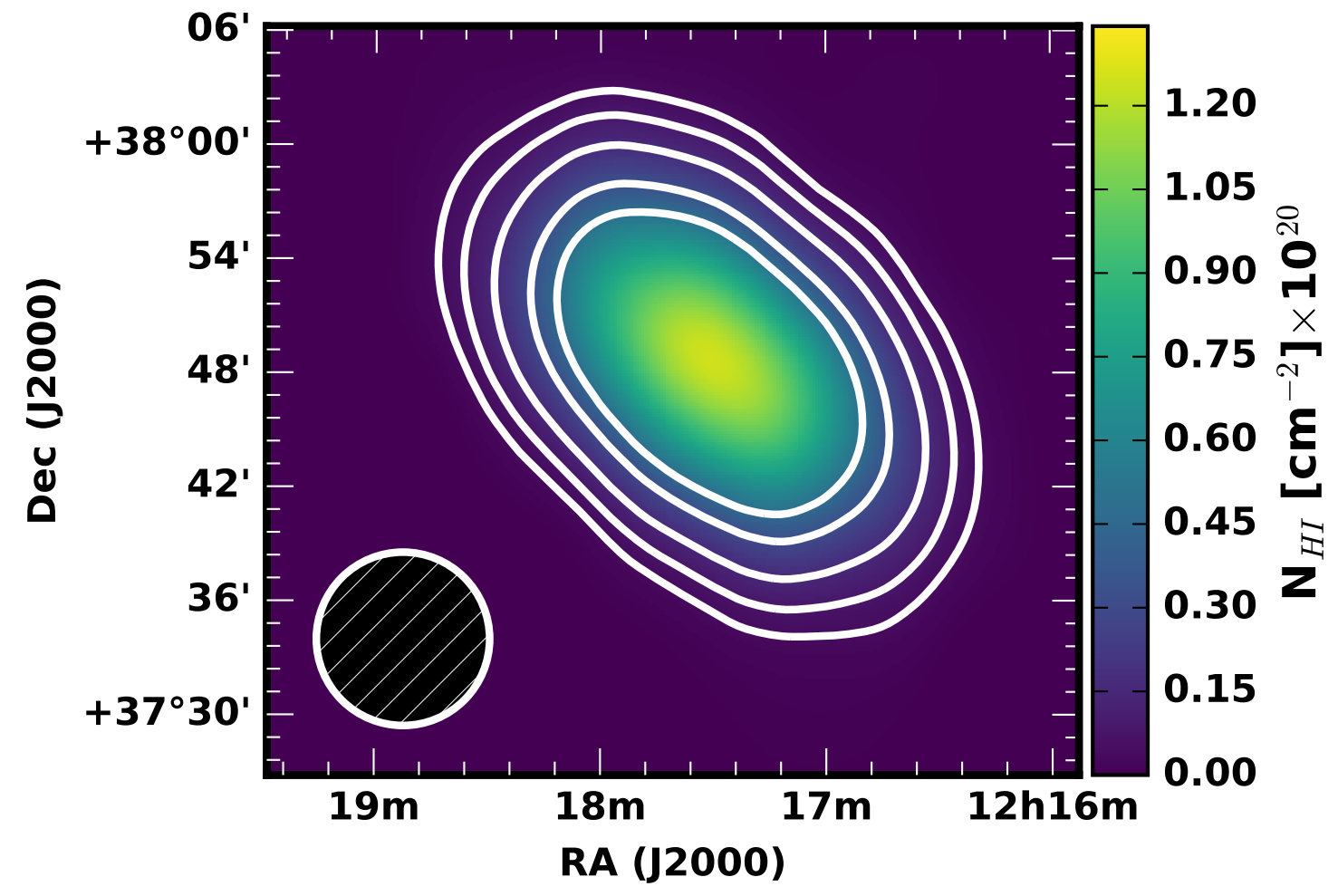

Figure 3.10 Integrated Hi intensity map of NGC4244. The contours are at the same levels quoted in Figure 3.6. 


\begin{tabular}{|c|c|c|c|c|c|}
\hline Source Pair & $M_{d y n}\left[M_{\odot}\right]$ & $M_{\text {comp }} M_{\odot}$ & $R_{\mathrm{sep}}[\mathrm{kpc}]$ & $R_{\text {gal }}[\mathrm{kpc}]$ & $Q$ \\
\hline NGC4258-N & $5.7 \times 10^{11} M_{\odot}$ & $1.4 \times 10^{11}$ & 150 & 92 & 0.059 \\
\hline $\mathrm{NG}$ & $5.7 \times 10^{11} M_{\odot}$ & & 205 & 92 & 0.0032 \\
\hline NGC4288-UGC7408a & $1.4 \times 10^{11} M_{\odot}$ & $2.0 \times 10^{9} M_{\odot}$ & 70 & 56 & 0.059 \\
\hline
\end{tabular}

Table 3.2 $Q$ Parameters for the interactions between NGC4258, NGC4288, and UGC7408

$$
{ }^{1} R_{\text {gal }} \text { of UGC7408 is } 48 \mathrm{kpc}
$$

\subsubsection{NGC4258}

The field of NGC4258 contains almost six companion galaxies labeled in the $N_{\mathrm{HI}}$ of the field shown in Figure 3.11. The must interesting feature is a prominent filament extending from NGC4288 pointing towards NGC4258. This filament was seen previously with the $76.2 \mathrm{~m}$ Lovell telescope at the Jodrell Bank Observatory (UK) as part of the Hi Jodrell All Sky Survey (HIJASS); (Wolfinger et al., 2013). Its classification in that survey was that of an 'Hi cloud' with the designation HIJASS J1219+46; no known optical counterparts are observed over the span of the HI emission.

The total flux of the feature is $(1.47 \pm 0.07) \mathrm{Jy} \mathrm{km} \mathrm{s}^{-1}$, which corresponds to an Hi mass of $(1.00 \pm 0.95) \times 10^{6} M_{\odot}$, assuming the filament is at the same distance of 7.6 Mpc as NGC4258, and a peak $N_{\mathrm{HI}}$ level of about $9.5 \times 10^{17} \mathrm{~cm}^{-2}$ in a single $5.2 \mathrm{~km} \mathrm{~s}^{-1}$ wide velocity channel.

Channel maps in Figures 3.12 and 3.13 shows the velocity range of the feature extends from $375 \mathrm{~km} \mathrm{~s}^{-1}$ to $422 \mathrm{~km} \mathrm{~s}^{-1}$.

The diffuse nature of the filament begs the question of origin. Its presence in a highly populated group of galaxies suggests the feature most likely originates from 
tidal perturbations. As a way to explore the this possibility, the Dahari parameter $^{1}$ (Dahari, 1984) is computed for the interactions between NGC4288-UGC7408, NGC4258-NGC4288, and NGC4258-UGC7408. Given their relatively large mass and close proximity to one another, these specific galaxy pairs are the most likely to produce such a diffuse tidal tail. The Darahi parameter $Q$ is defined to as

$$
Q=\left(\frac{M_{c}}{M_{\text {gal }}}\right) \cdot\left(\frac{R_{\mathrm{sep}}}{R_{\text {gal }}}\right),
$$

where $M_{c}$ is the mass of the companion, $M_{g a l}$ is the mass of the galaxy under consideration (both taken to be the respective dynamical masses), $R_{\text {sep }}$ is the projected physical separation, and $R_{\text {gal }}$ is the radius of the galaxy (computed out to the $3 \sigma$ $N_{\mathrm{HI}}$ contour. The results of this analysis are shown in Table 3.2. Note that the dynamical masses are computed via the equation $M_{d y n}=2.33 \times 10^{5} R_{\text {gal }} V_{\text {rot }}^{2}$, where $V_{\text {rot }}$ is the rotation velocity taken from a Hyperleda search. The highest $Q$ value of 0.059 corresponds to the interaction between NGC4258 and NGC4288, is likely strong enough to say these galaxies are interacting at some level (Dahari, 1984), but perhaps not strong enough to produce dramatic effects such as a diffuse tidal tail. The interaction between NGC4258 and UGC7408 is orders of magnitude too weak to produce the Hi filament. That said, more in-depth modeling is required to be sure. A restricted 3-body code that can easily be run on a modern laptop so as to not be limited by large computation times, such as JSPAM (Wallin et al., 2016), could be employed to constrain the exact origin scenarios.

\footnotetext{
${ }^{1}$ This is effectively the tidal force per unit mass.
} 
The unique structure of NGC4258 consists of two anomalous spiral arms that extending through the optical disk (van der Kruit et al., 1972), otherwise the disk behaves without evidence of disruption. To avoid contamination from the other companion galaxies and focus on the diffuse Hi specifically associated with the filament, a portion of the Hi distribution shown in Figure 3.11 is extracted for use in the $N_{\mathrm{HI}}$ analysis and is shown in Figure 3.14.

\subsubsection{NGC4559}

The estimated mass fraction of the extraplanar Hi gas of NGC4559 is between about $10 \%$ to $20 \%$ as revealed by tilted-ring models fit to the HALOGAS WSRT data (Vargas et al., 2017). The origin of this is extraplanr component is linked to the star formation with a vertical rotational velocity gradients similar to those found in NGC891 Oosterloo et al. (2007), NGC2403 Fraternali et al. (2002), and NGC4565 Zschaechner et al. (2011). The GBT data cube does not show any hints of a distinct diffuse Hi feature that may be associated with this extraplanar gas component. The $N_{\mathrm{HI}}$ map is shown in Figure 3.15.

\subsubsection{UGC7774}

High-resolution HI data reveal a strong warp is present towards the outer disk regions of this source (García-Ruiz et al., 2002). However, the $N_{\mathrm{HI}}$ map in Figure 3.16 shows no hints of strong morphological asymmetries larger than the GBT beam. While there are several companions towards the southwest edge of the 


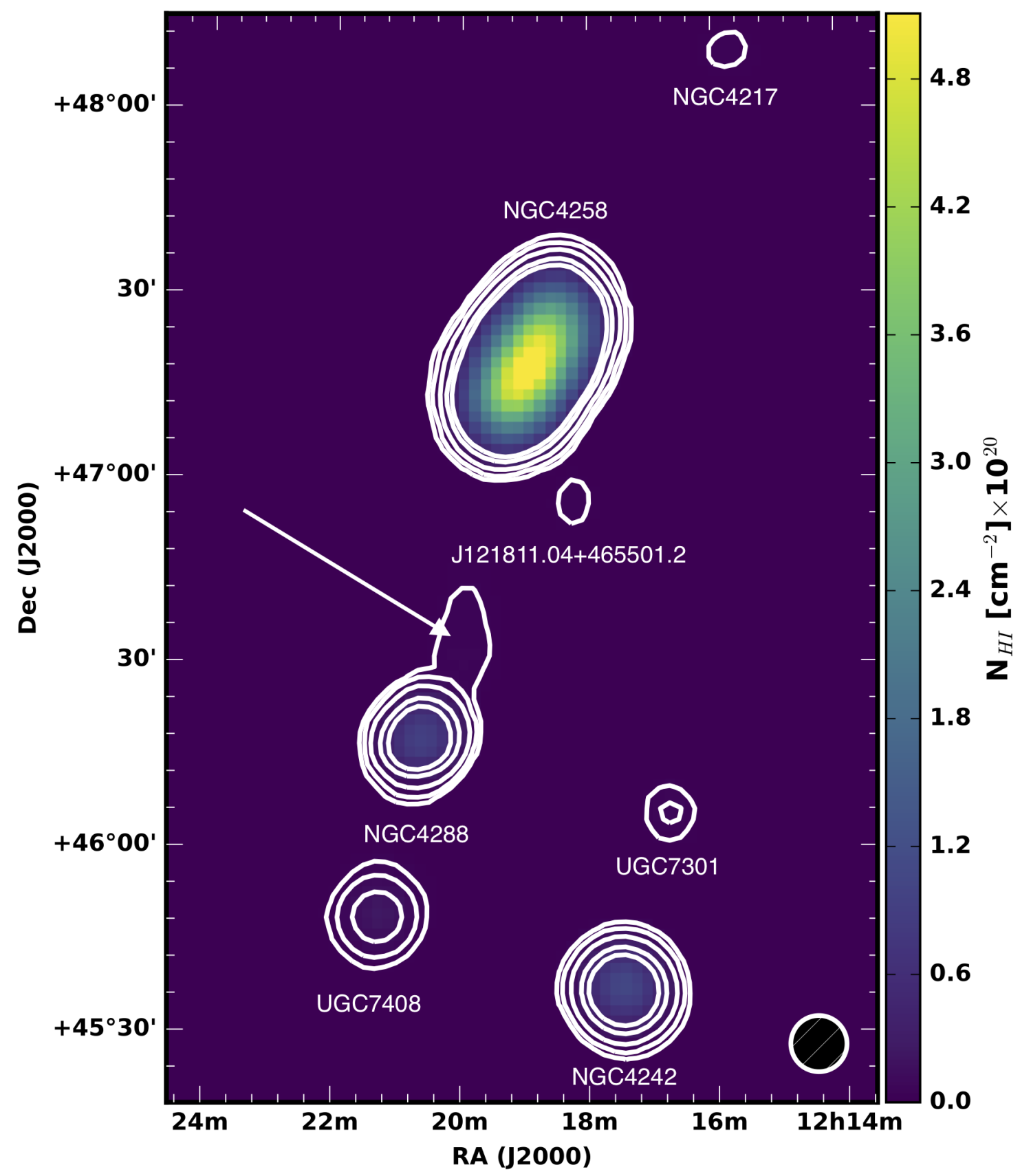

Figure $3.11 N_{\mathrm{HI}}$ map of NGC4258 field. The contours are at the same levels quoted in Figure 3.6. 

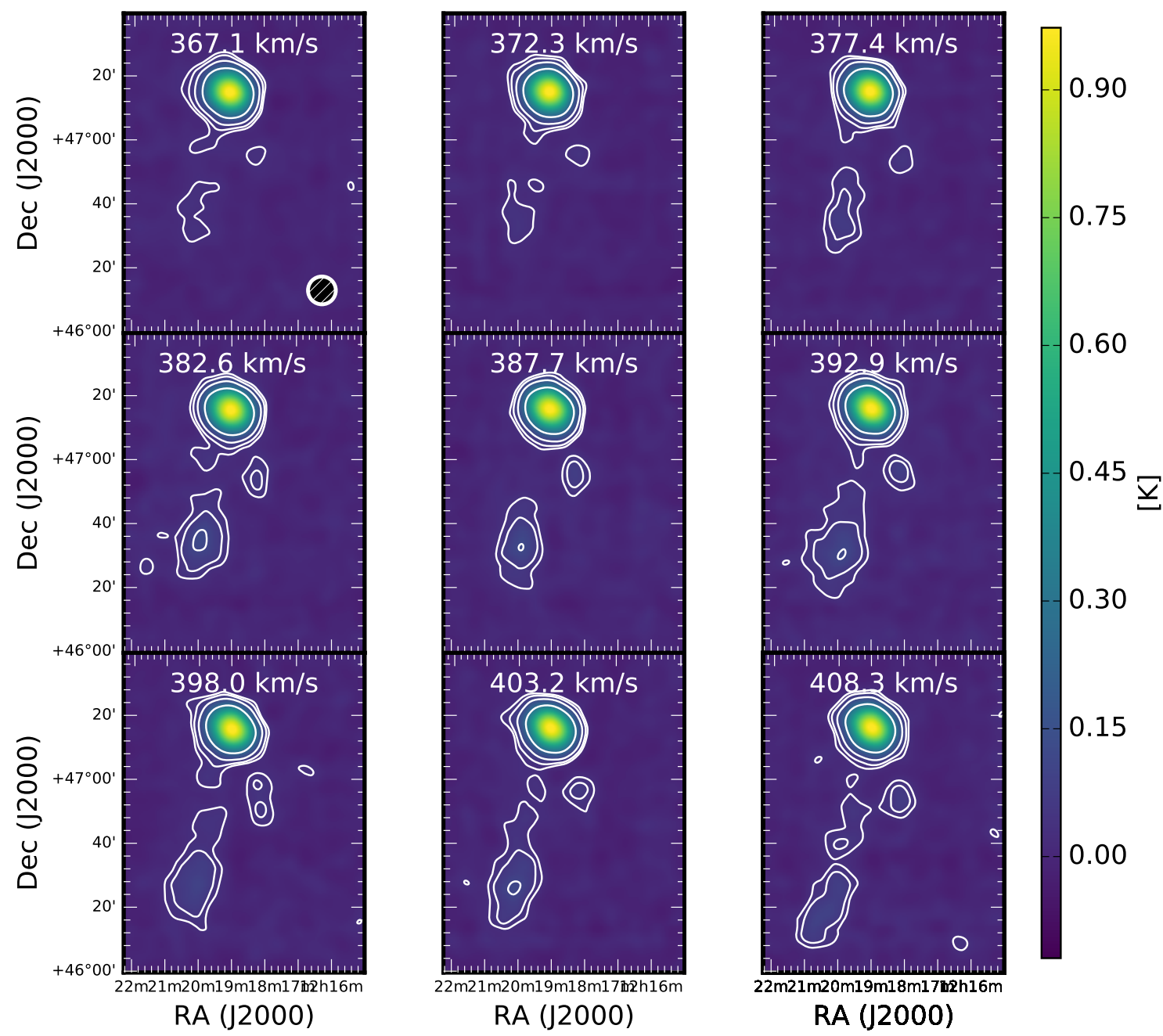

Figure 3.12 Contour maps of several NGC4258 velocity channels in the GBT cube. The white contours begin at $3 \sigma$ in one $5.2 \mathrm{~km} \mathrm{~s}^{-1}$ velocity channel $\left(3.4 \times 10^{17} \mathrm{~cm}^{-2}\right)$ and continue at 5, 10, and 25 times that level. 

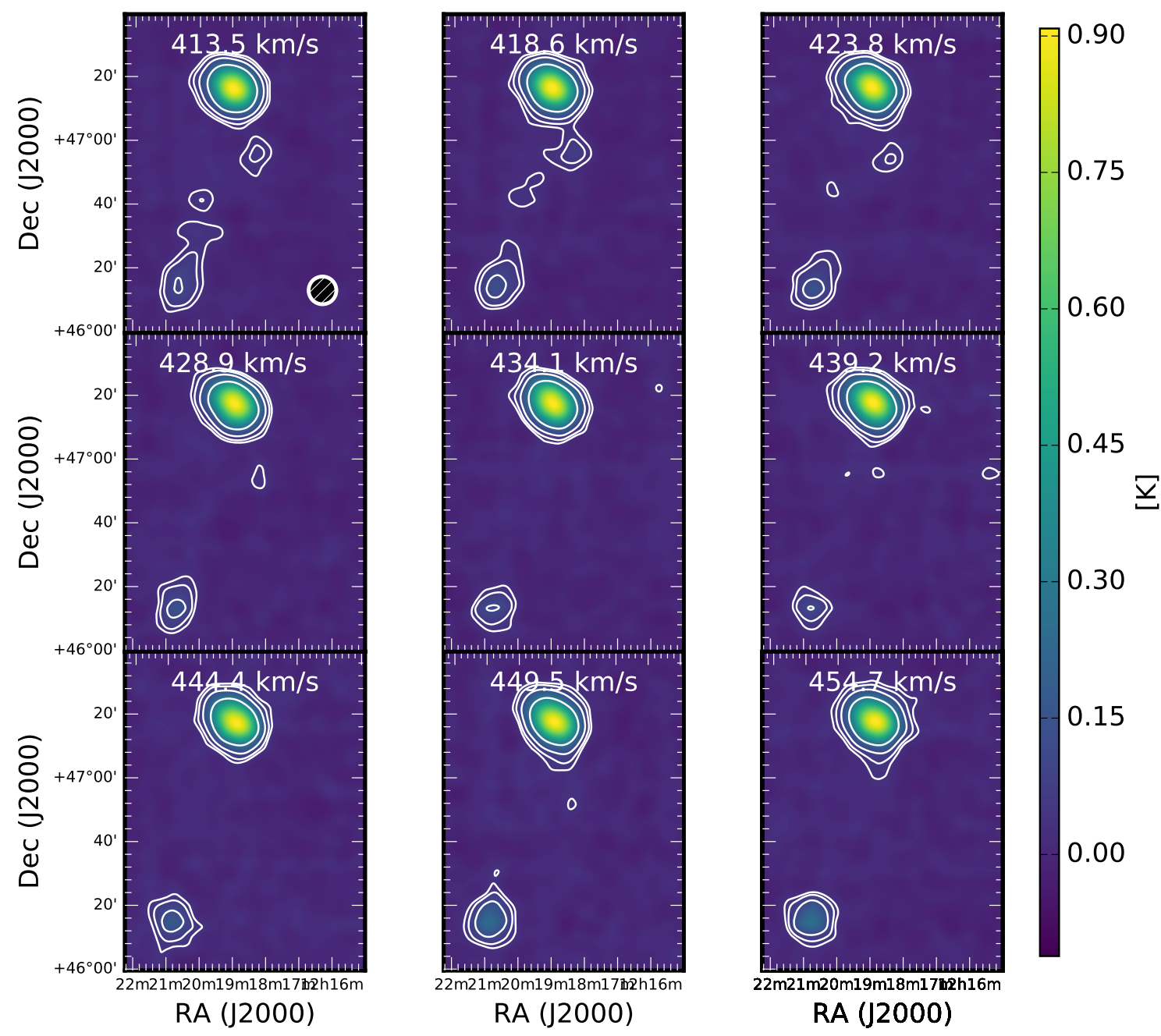

Figure 3.13 Contour maps of several NGC4258 velocity channels in the GBT cube. The white contours are at the levels denoted in the caption for Figure 3.12. 


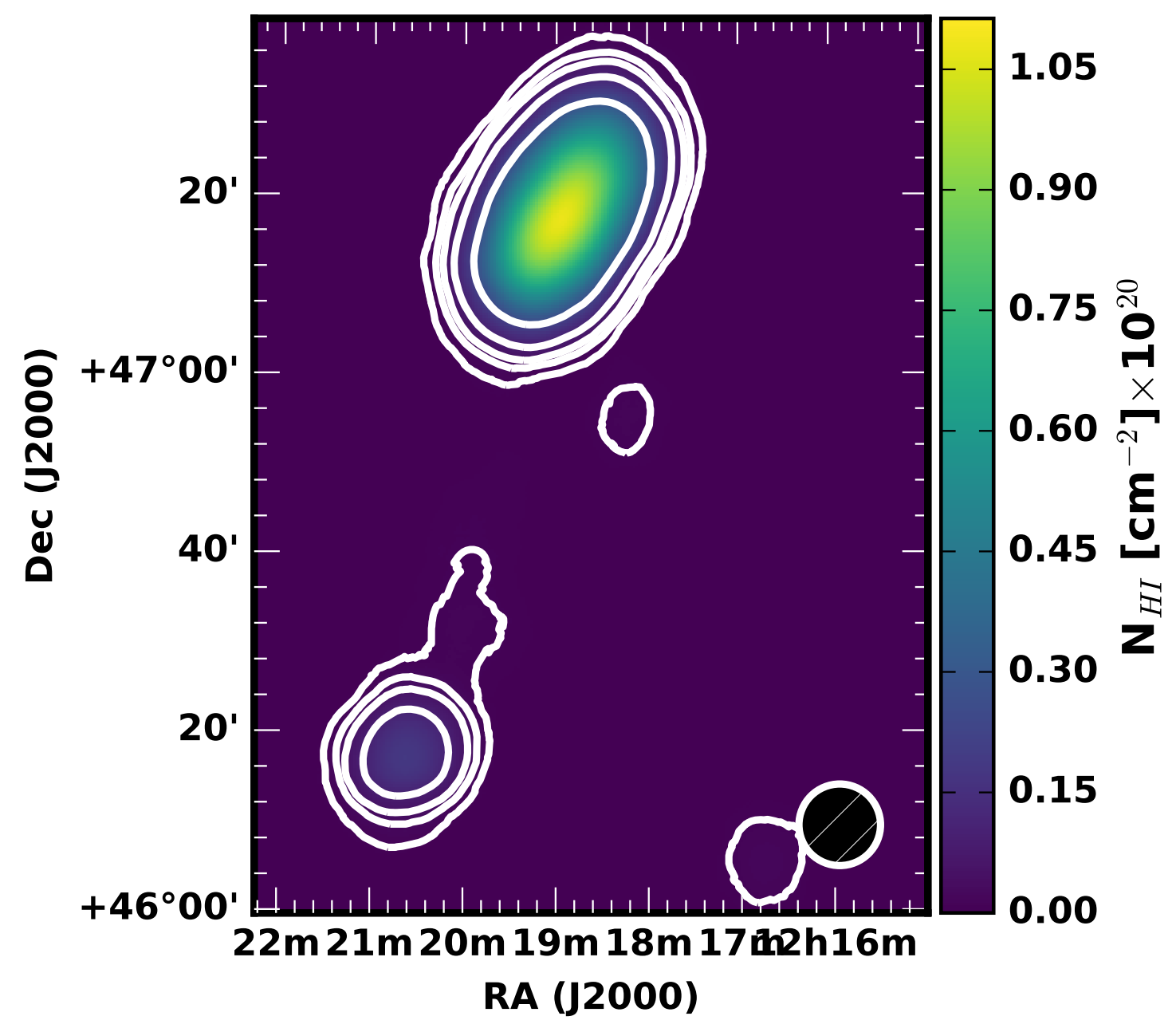

Figure 3.14 Region of the $N_{\mathrm{HI}}$ map of NGC4258 extracted for analysis to focus on the diffuse $\mathrm{HI}$ associated with the filament. The contours begin at a $3 \sigma N_{\mathrm{HI}}$ level of $1 \times 10^{18} \mathrm{~cm}^{-2}$ and continue at 5, 10, and 25 times that level. 


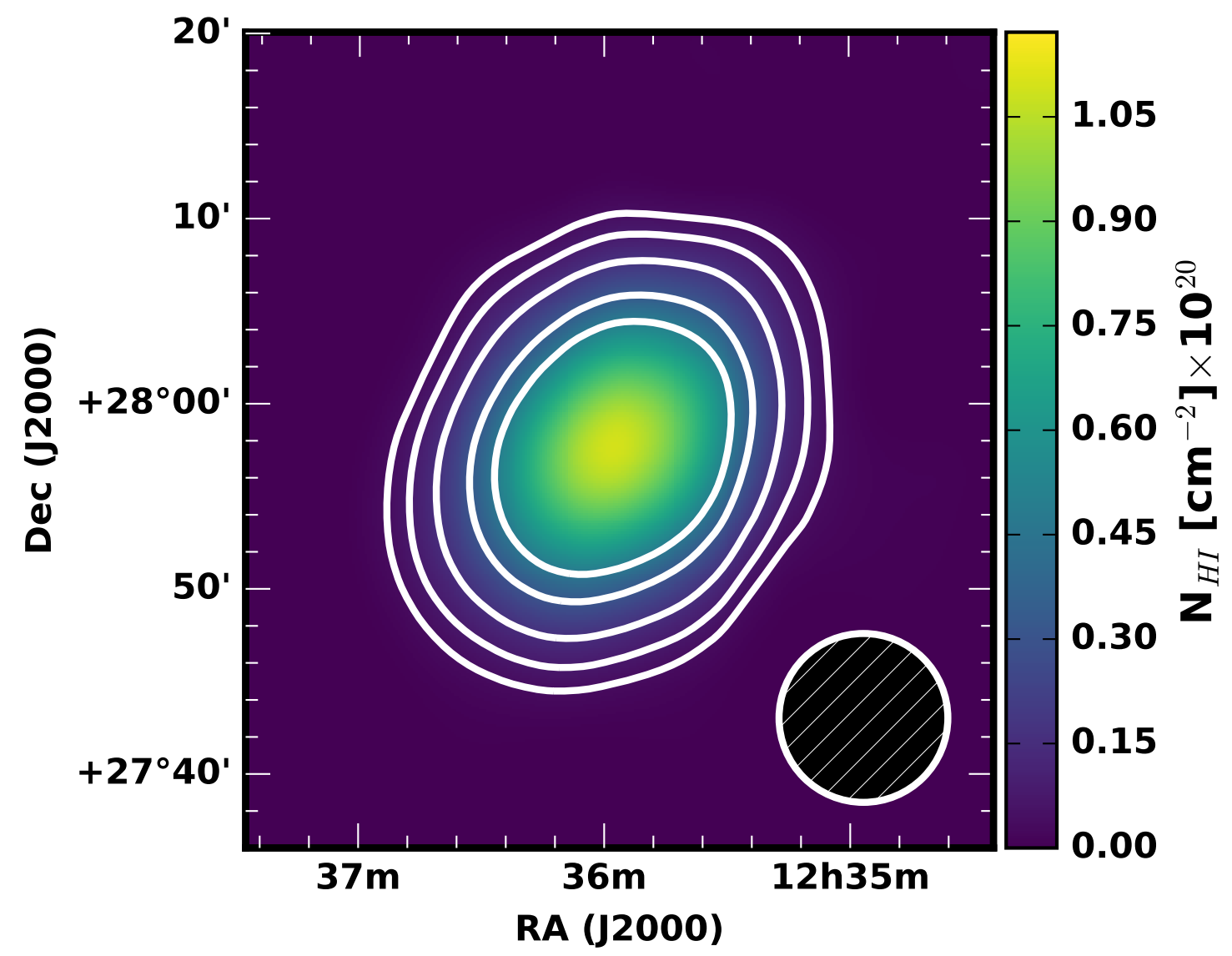

Figure $3.15 N_{\mathrm{HI}}$ map of NGC4559. The contours are at the same levels quoted in Figure 3.6. 


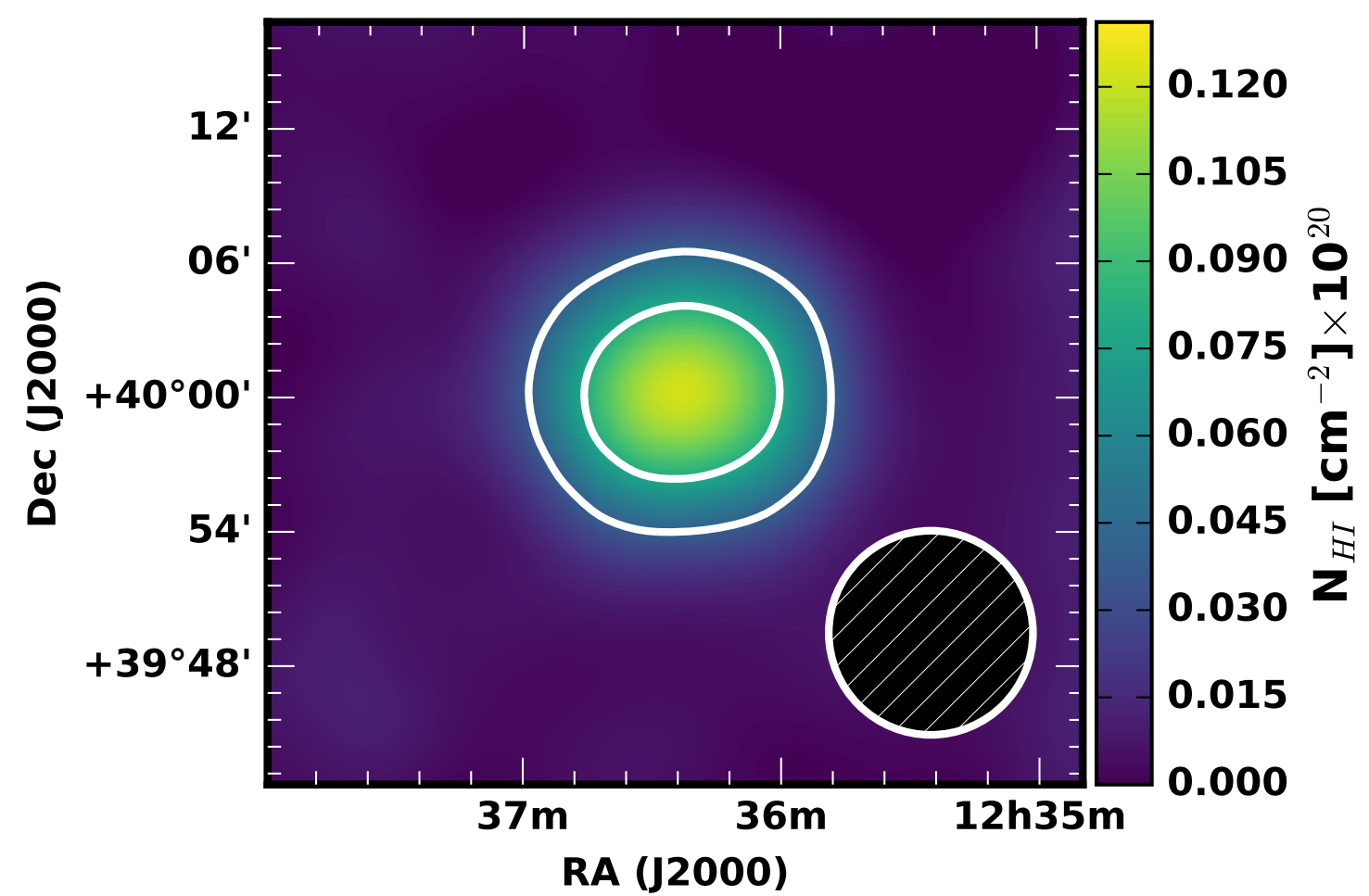

Figure $3.16 N_{\mathrm{HI}}$ map of UGC7774. The contours are at the same levels quoted in Figure 3.6.

GBT map, there is no indication of any large-scale diffuse connection in HI.

\subsubsection{NGC4631}

NGC4631 (Figure 3.17) is known to be an interacting with its nearby companions to the southeast (NGC4656) and to the north (NGC4627) with four distinct Hi extensions off of its main disk Hi distribution (Weliachew et al., 1978). The observed Hi distribution can be completely reproduced with numerical simulations of the tidal interactions between galaxies (Combes, 1978).

No connection in Hi between these galaxies has been observed in previous high-resolution data. The GBT $N_{\mathrm{HI}}$ map in Figure 3.17 shows for the first time 
that the system sits within a common Hi envelope that has asymmetrical features several times larger than the beam size. Channel maps in Figures 3.18 to 3.21 show

connecting Hi between NGC4631 and NGC4627 that persists over $200 \mathrm{~km} \mathrm{~s}^{-1}$. A distinct Hi feature to the northeast is seen at around $600 \mathrm{~km} \mathrm{~s}^{-1}$ and remains prominent to almost $740 \mathrm{~km} \mathrm{~s}^{-1}$ in radial velocity. The $0.5^{\circ}$ (or about $66 \mathrm{kpc}$ in projected physical length) over which the feature spans over only $\sim 150 \mathrm{~km} \mathrm{~s}^{-1}$ suggests it is a tidal tail resulting from the gravitational interactions between NGC4631 and NGC4627. This is likely an extension of the Hi spurs 3 and 4 originally seen by Rand (1994). Combes (1978) postulated that while NGC4627 is currently a dwarf elliptical galaxy, it had once been an irregular or spiral galaxy before its interaction with the $50 \times$ more massive NGC4631. Similar to formation scenarios of the Magellanic Bridge (e.g., Putman 2000), this tidal feature could consist of the gas stripped from NGC4627. Such a well-resolved interacting system provides a very interesting test for the analysis methods since Hi envelope likely contains a large fraction of diffuse Hi than the typical isolated galaxies examined thus far.

\subsubsection{NGC5023}

The HI distribution in NGC5023 is found in Figure 3.22 to be asymmetric in the high angular resolution WSRT data (Kamphuis et al., 2013), attributed to the presence of a warp along the line of sight, in addition to the presence of several extraplanar gas features. The GBT data do not reveal any indications that the large scale HI distribution is disturbed, or the presence of diffuse features that may 


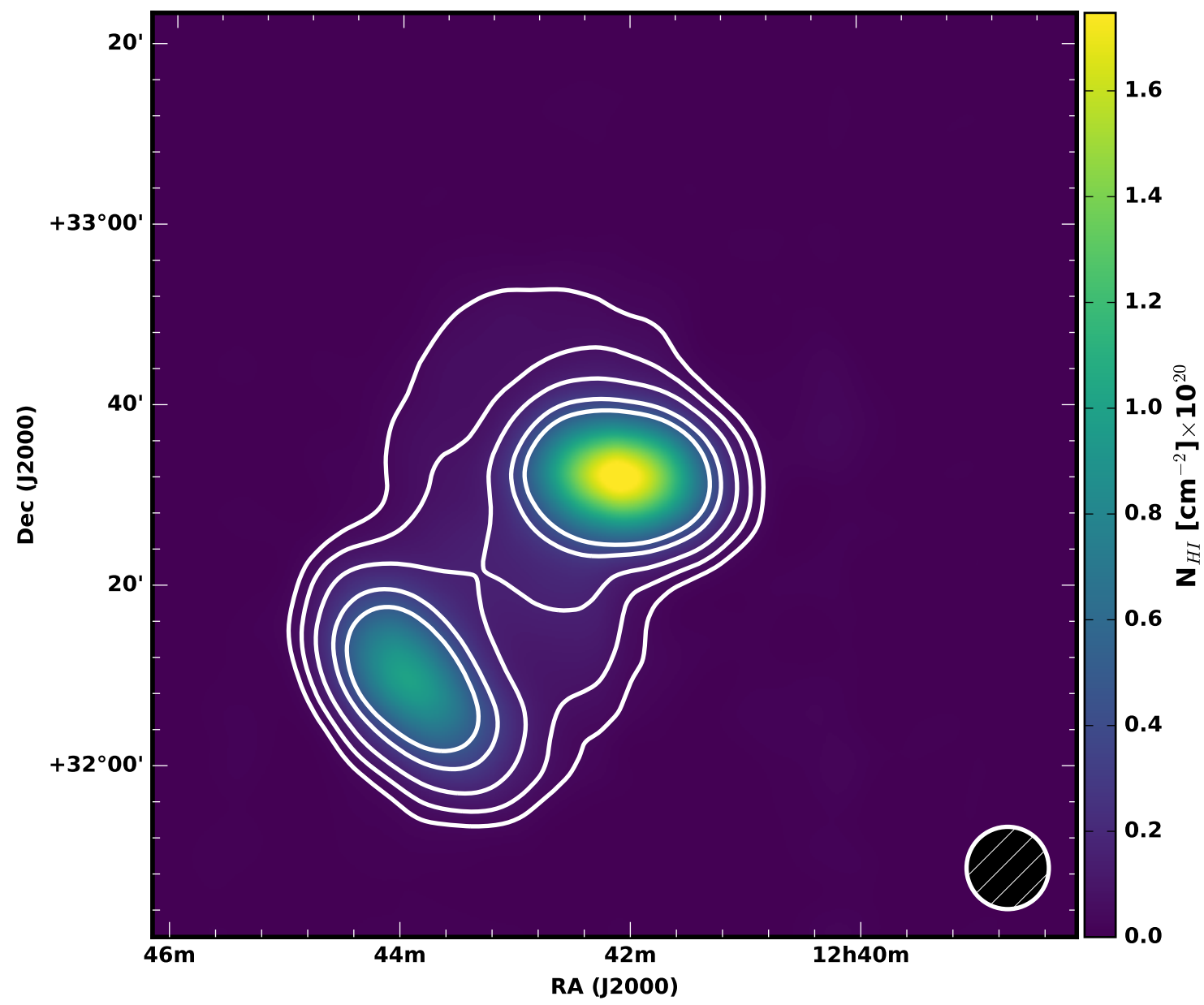

Figure $3.17 N_{\mathrm{HI}}$ map of NGC4631. The contours are at the same levels quoted in Figure 3.6.) 

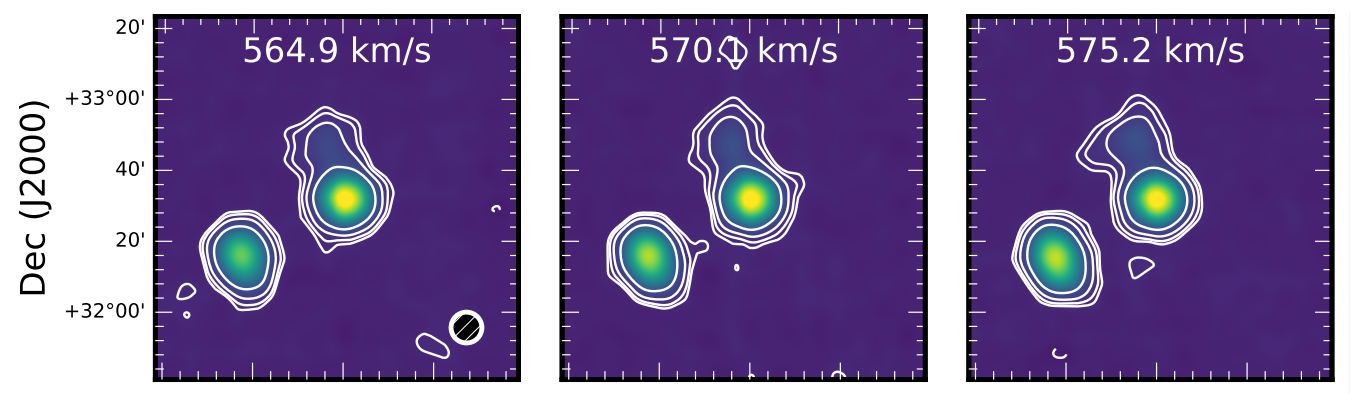

1.6
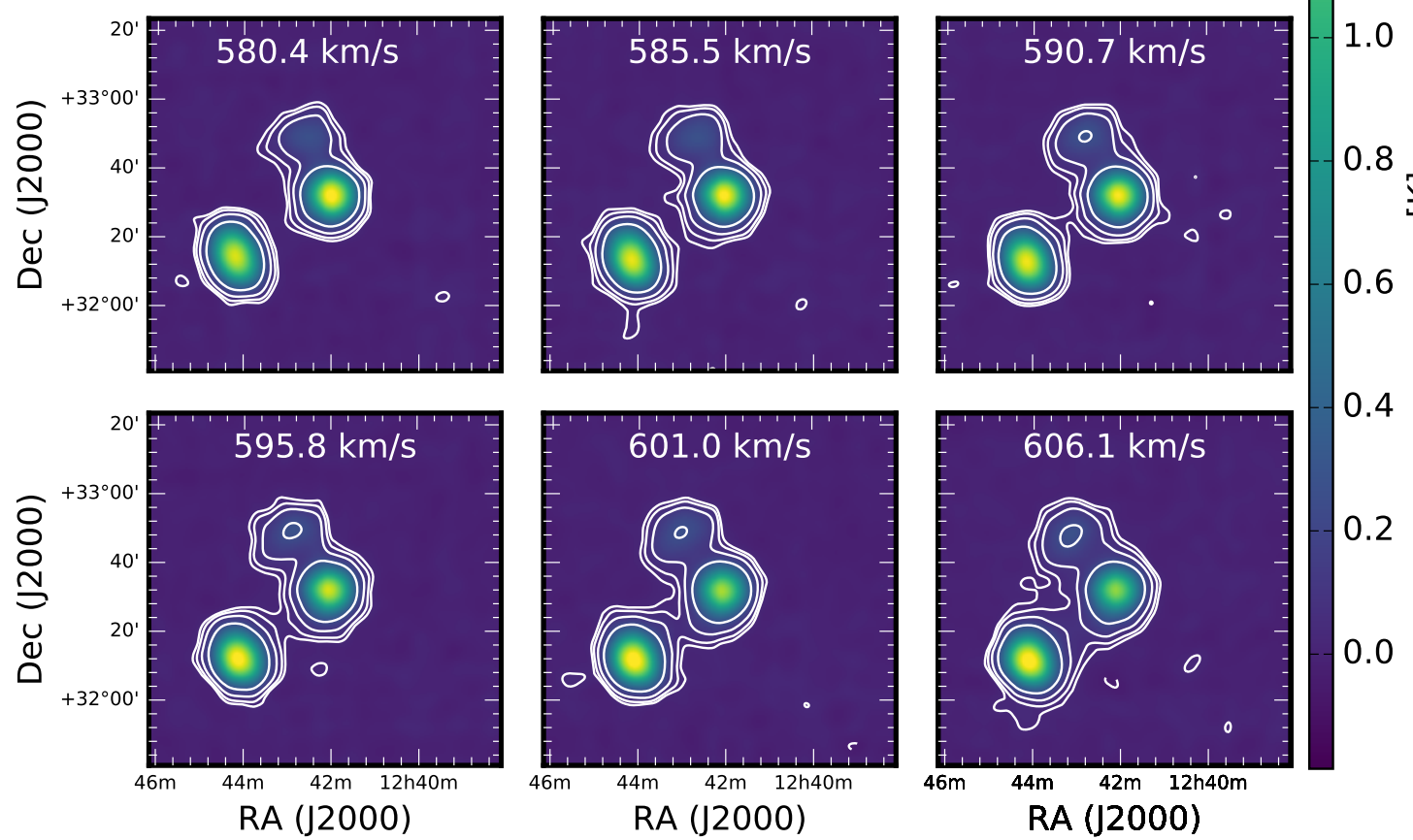

Figure 3.18 Contour maps of selected velocity channels in the GBT data cube for NGC4631. The white contours begin at $3 \sigma$ in one $5.2 \mathrm{~km} \mathrm{~s}^{-1}$ velocity channel $\left(3.4 \times 10^{17} \mathrm{~cm}^{-2}\right)$ and continue at 5, 10, and 25 times that level. 

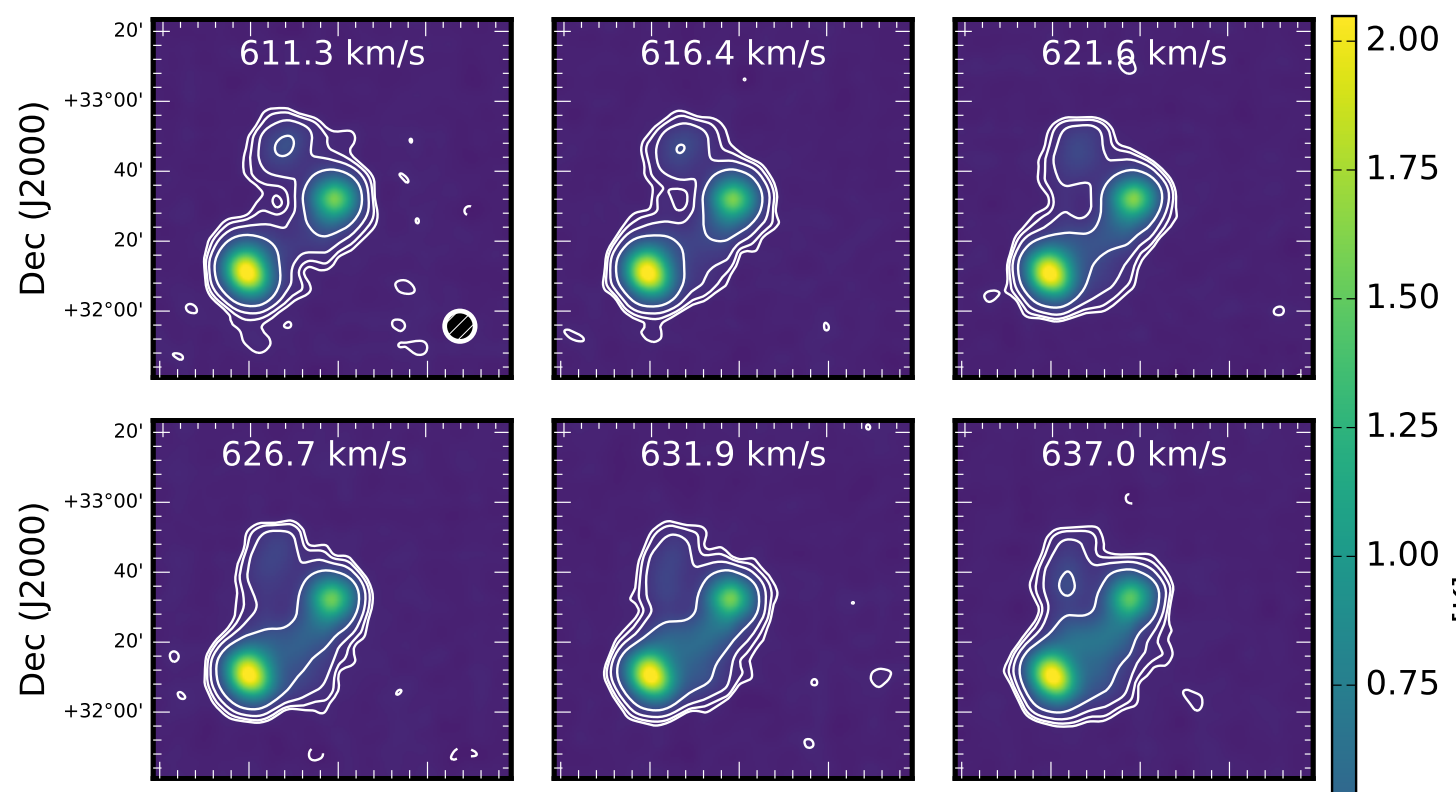

1.25
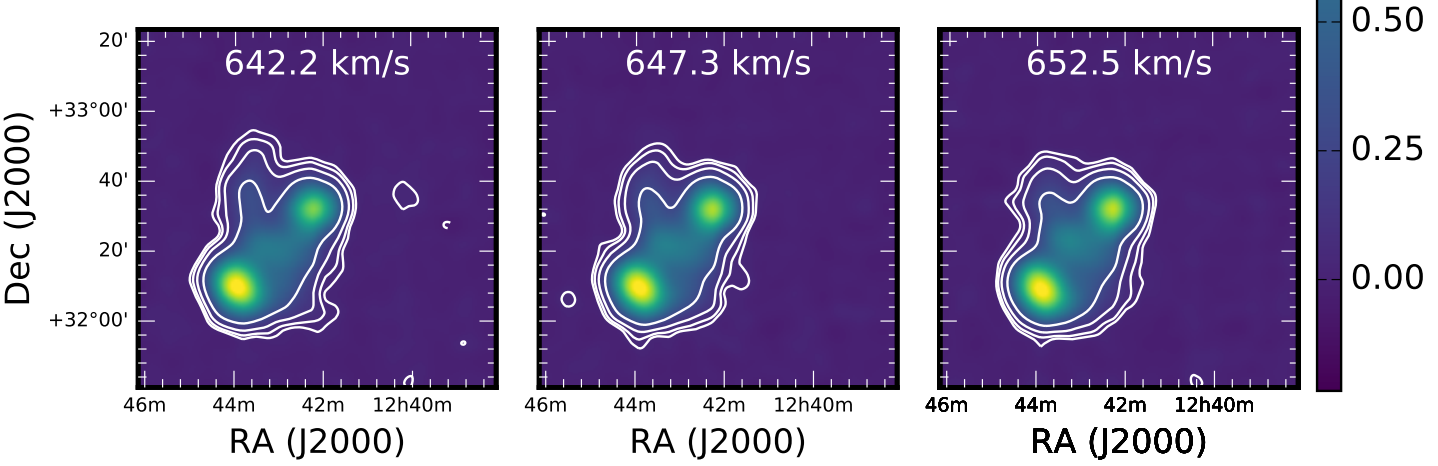

Figure 3.19 Contour maps of selected velocity channels in the GBT data cube for NGC4631. The contour levels are the same as quoted in the caption for Figure 3.18. 

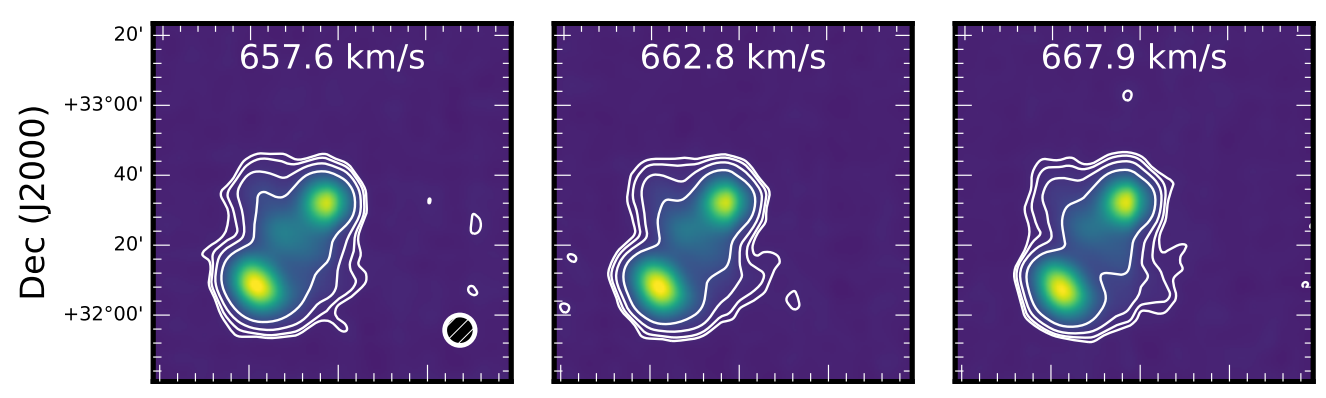

2.00
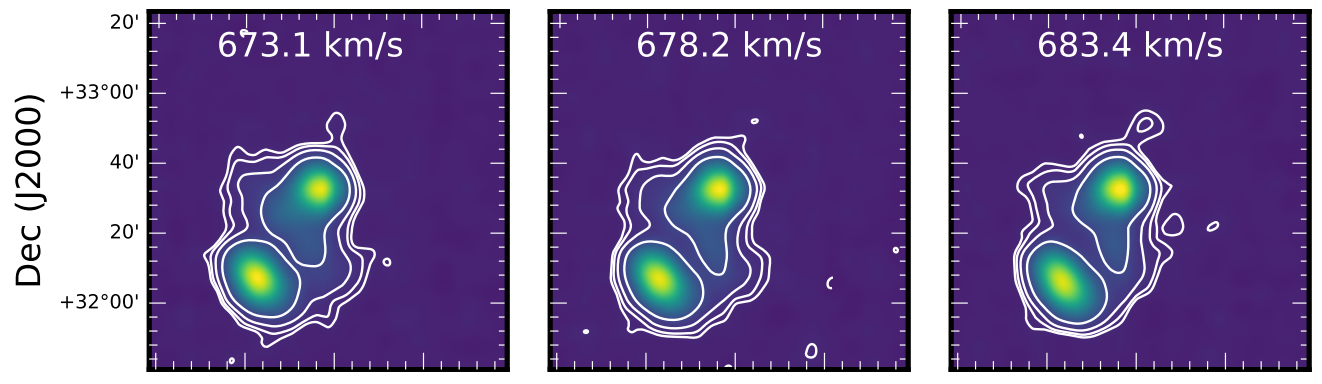

1.75

1.50
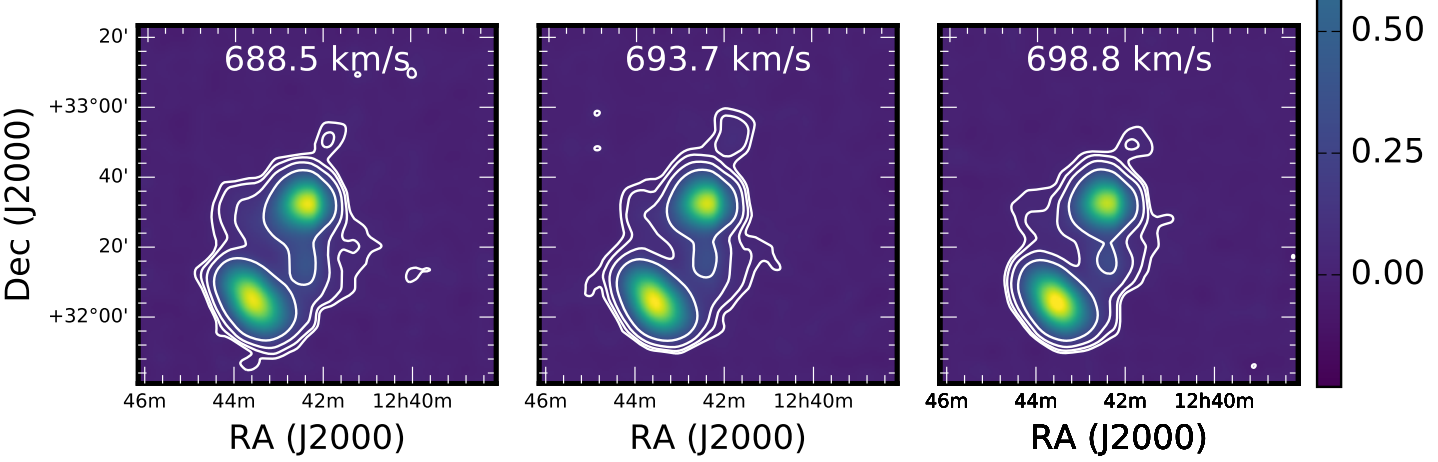

Figure 3.20 Contour maps of selected velocity channels in the GBT data cube for NGC4631. The contour levels are the same as quoted in the caption for Figure 3.18. 

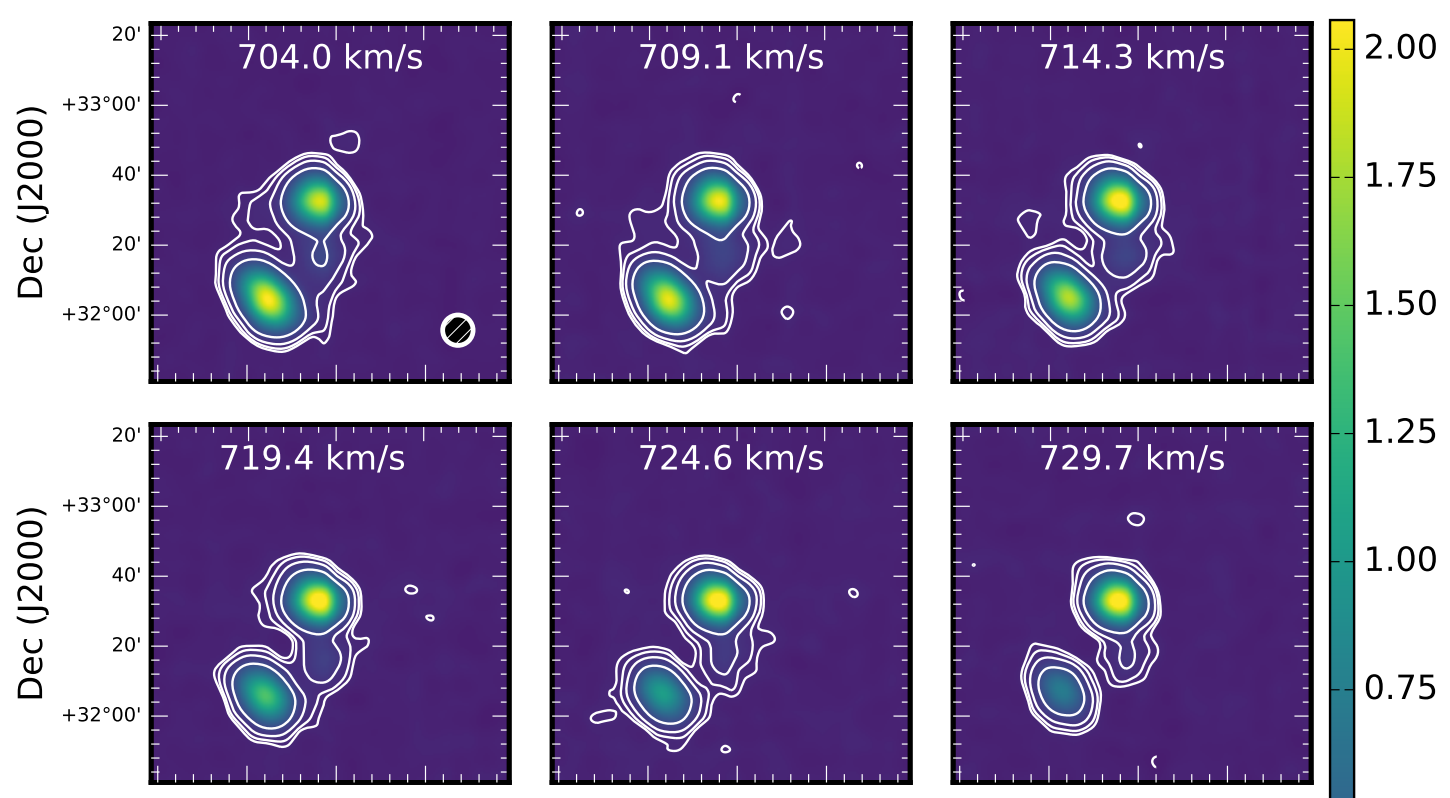

1.25

1.50
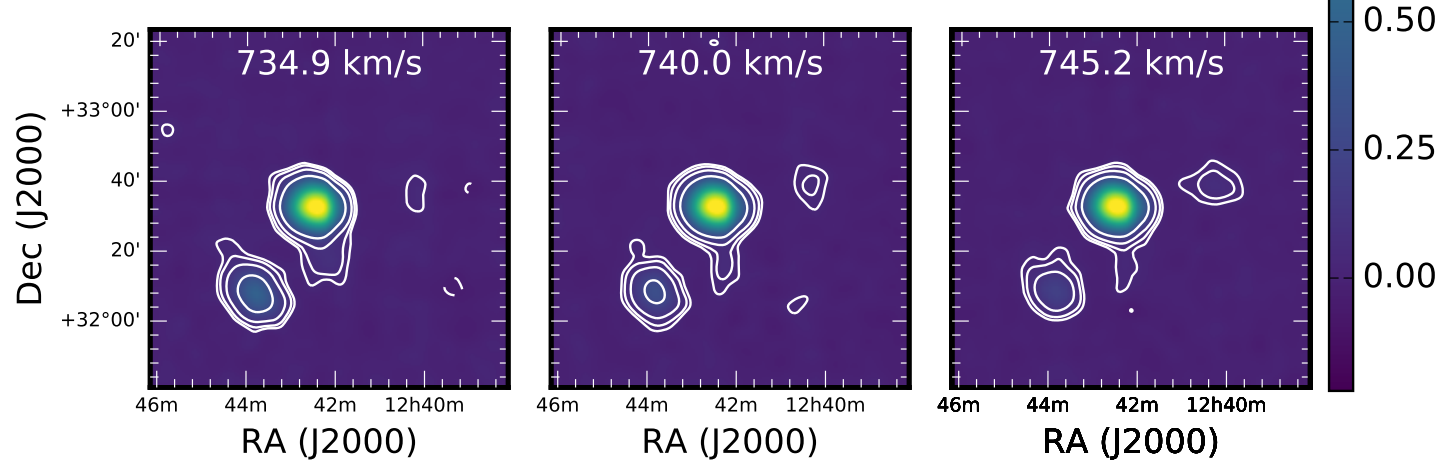

Figure 3.21 Contour maps of selected velocity channels in the GBT data cube for NGC4631. The contour levels are the same as quoted in the caption for Figure 3.18. 


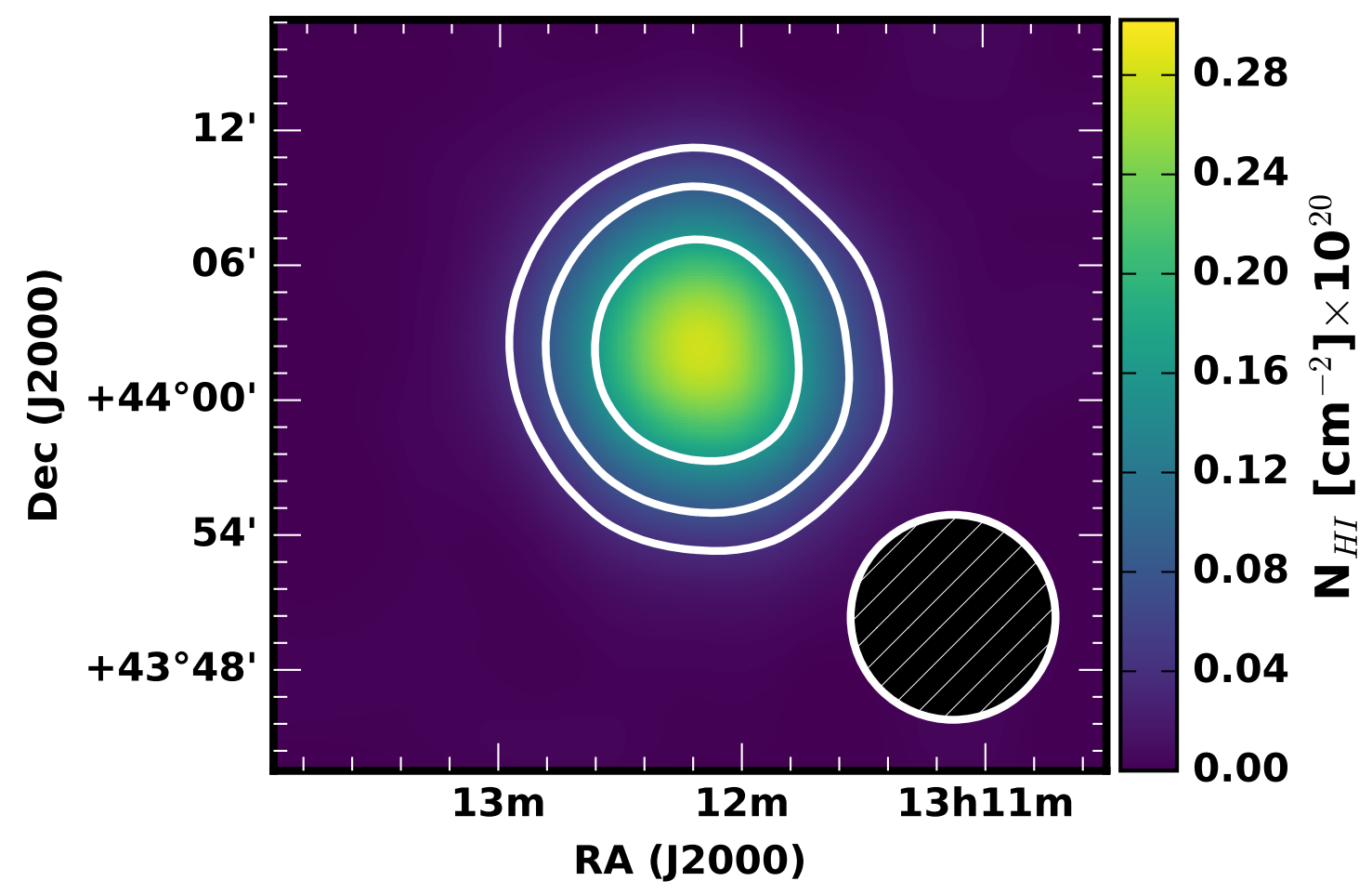

Figure $3.22 N_{\mathrm{HI}}$ map of NGC5023. The contours are at the same levels quoted in Figure 3.6.

be associated with the extraplanar Hi component in individual channel maps (not shown).

\subsubsection{NGC5229}

The $N_{\mathrm{HI}}$ map in Figure 3.23 shows that the extent of the large scale Hi distribution source is not much larger than the GBT beam; thus, this source is considered to be only marginally resolved. Beyond a minor warp, the HI in NGC5229 is distributed in symmetrical disk (George Heald; private communication). Again, the GBT data do not indicate any evidence for diffuse features that could be associated this source. 


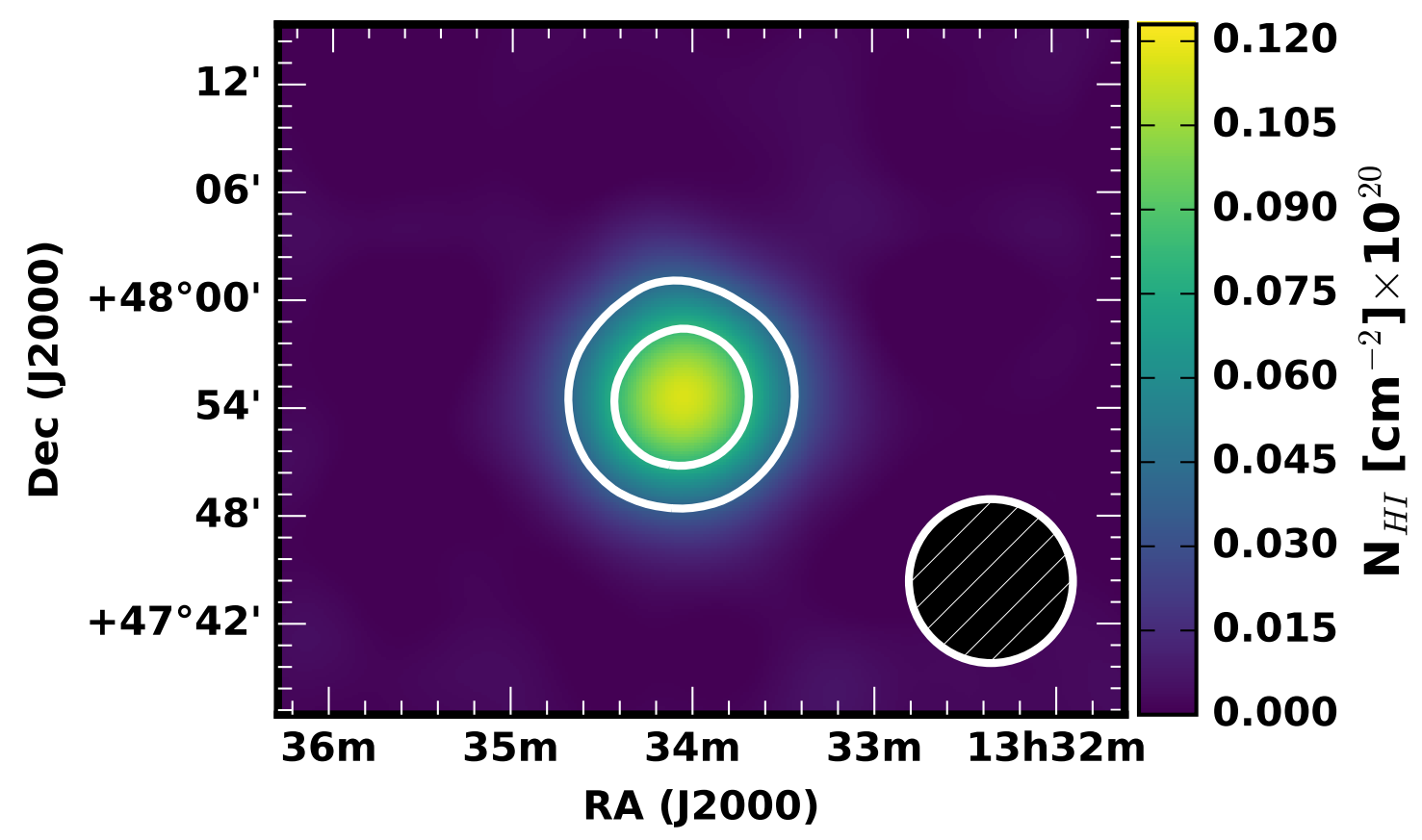

Figure $3.23 N_{\mathrm{HI}}$ map of NGC5229. The contours are at the same levels quoted in Figure 3.6.

\subsubsection{NGC5585}

The final source to be included in the analysis is shown to be well-resolved within the GBT beam (Figure 3.24). Channel maps do not show any indications for diffuse features. The GBT field contains only one other known companion, designated SDSS J141510.07+570515.3 at a position of $\alpha_{J 2000}=14^{h} 15^{m} 10.070^{s}$, $\delta_{J 2000}=+57^{\circ} 05^{\prime} 15.33^{\prime \prime}$, with no evidence for an interaction with NGC5585.

\subsection{Analysis}

The plots (Figures 3.25 to 3.36) that follow summarize the analysis that was introduced in Chapter 2. In these four panel figures for each galaxy, the top left panel shows the Hi flux as a function of velocity (panel a); the top right panel presents 


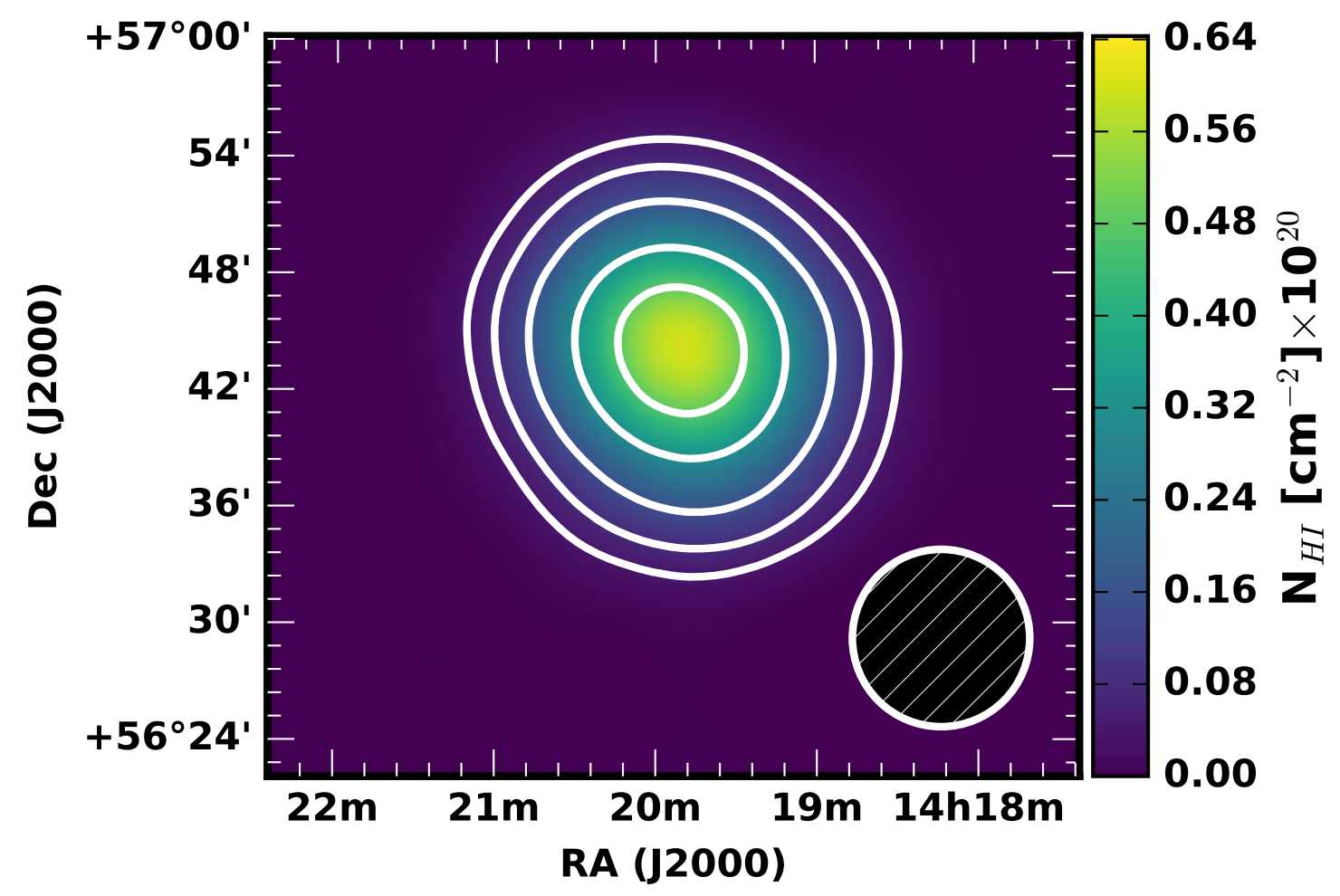

Figure 3.24 Integrated Hi intensity map of NGC5585. The contours are at the same levels quoted in Figure 3.6. 
the cumulative Hi mass as a function of $N_{\mathrm{HI}}$ (panel b); the bottom left panel shows the radial mean (averaged in azimuthal bins) $N_{\mathrm{HI}}$ profile as a function of projected physical radius (panel c); and the bottom right panel expresses the cumulative flux as a function of projected physical radius (panel d). Marginally resolved, sources with potential image artifacts, or where only upper limits are available for the total flux will be discussed individually before moving on to the general trends of the sample as a whole.

\subsubsection{General Trends}

For galaxies to be included in the $f_{19}$ analysis, physical $N_{\mathrm{HI}}$ are required, which necessitates the sources be well-resolved. A signature of a well-resolved galaxy in the presented analysis is that the cumulative Hi mass distribution will lie above the profile of the GBT beam model (basically simulating the observation of an unresolved source); this must also be true of the azimuthally averaged $N_{\mathrm{HI}}$ values as a function of projected physical radius. Additionally, the cumulative flux as a function of projected physical radius should show no variations at large projected physical radii that would indicate the presence of artifacts.

The cumulative Hi mass as a function of $N_{\mathrm{HI}}$ can also reveal several characteristics about the a given galaxy's Hi distribution. Since this quantity is a measure of the HI contained at and above a given $N_{\mathrm{HI}}$ bin, a relatively flat profile indicates much of the Hi is contained at column densities that should be detectable in the

WSRT HALOGAS data (i.e., $N_{H I} \geq 10^{19} \mathrm{~cm}^{-2}$ ). NGC672 in Figure 3.25 is an il- 
lustrative example. Here, the cumulative Hi mass function is situated significantly higher than GBT beam model, demonstrating that this source is very well-resolved even in the relatively large GBT beam. Furthermore, the slope of the profile remains effectively constant and remains above 0.9 until $N_{\mathrm{HI}} \sim 10^{20.3} \mathrm{~cm}^{-2}$, revealing that the amount of Hi in column densities below $10^{19} \mathrm{~cm}^{-2}$ is a few percent of the total Hi mass. If a significant fraction of the HI were found to be in low column density bins, the shape of the cumulative Hi mass functions will show much more variation at the associated column density bins. In general, the profile of the cumulative Hi mass as a function of $N_{\mathrm{HI}}$ for the well-resolved sources show little variation for column densities between $10^{18} \mathrm{~cm}^{-2}$ and $10^{19} \mathrm{~cm}^{-2}$.

In addition to helping show a source is well-resolved, the azimuthally averaged $N_{\mathrm{HI}}$ profiles are useful for locating potential breaks that are predicted by photoionization models. Again, using NGC2403 as an example, there is no evidence of a break in the radial $N_{\mathrm{HI}}$ distribution, which would indicate a photoionization edge. Other sources such as NGC1003 in Figure 3.26 do show sudden variations at the lower column density levels, however. Inspection of the high-resolution WSRT data for similar behavior of the radial $N_{\mathrm{HI}}$ profile would help establish the reality of such a feature, perhaps revealing properties of the interface between the disk edge of the CGM. 

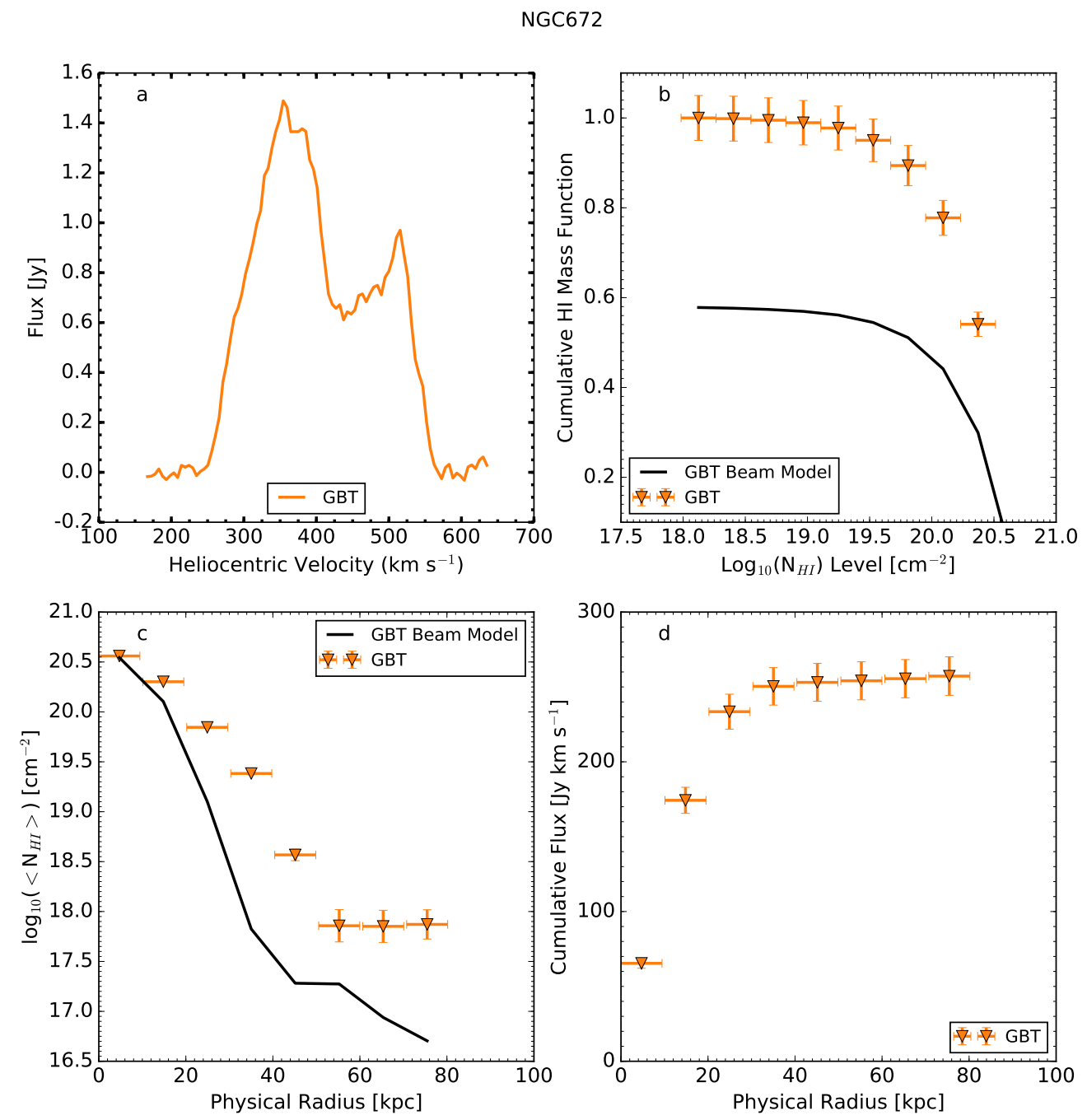

Figure 3.25 Summary of the various properties derived from the regridded GBT (orange inverted triangles) data set of NGC672. $a$ : global Hi profile; $b$ : Cumulative Hi mass as a function of $N_{\mathrm{HI}}$. The solid black line simulates an unresolved observation with our GBT beam model; $c$ : projected physical radial dependence on the azimuthally averaged $N_{\mathrm{HI}}$; $d$ : projected radial dependence of the cumulative flux 
NGC1003
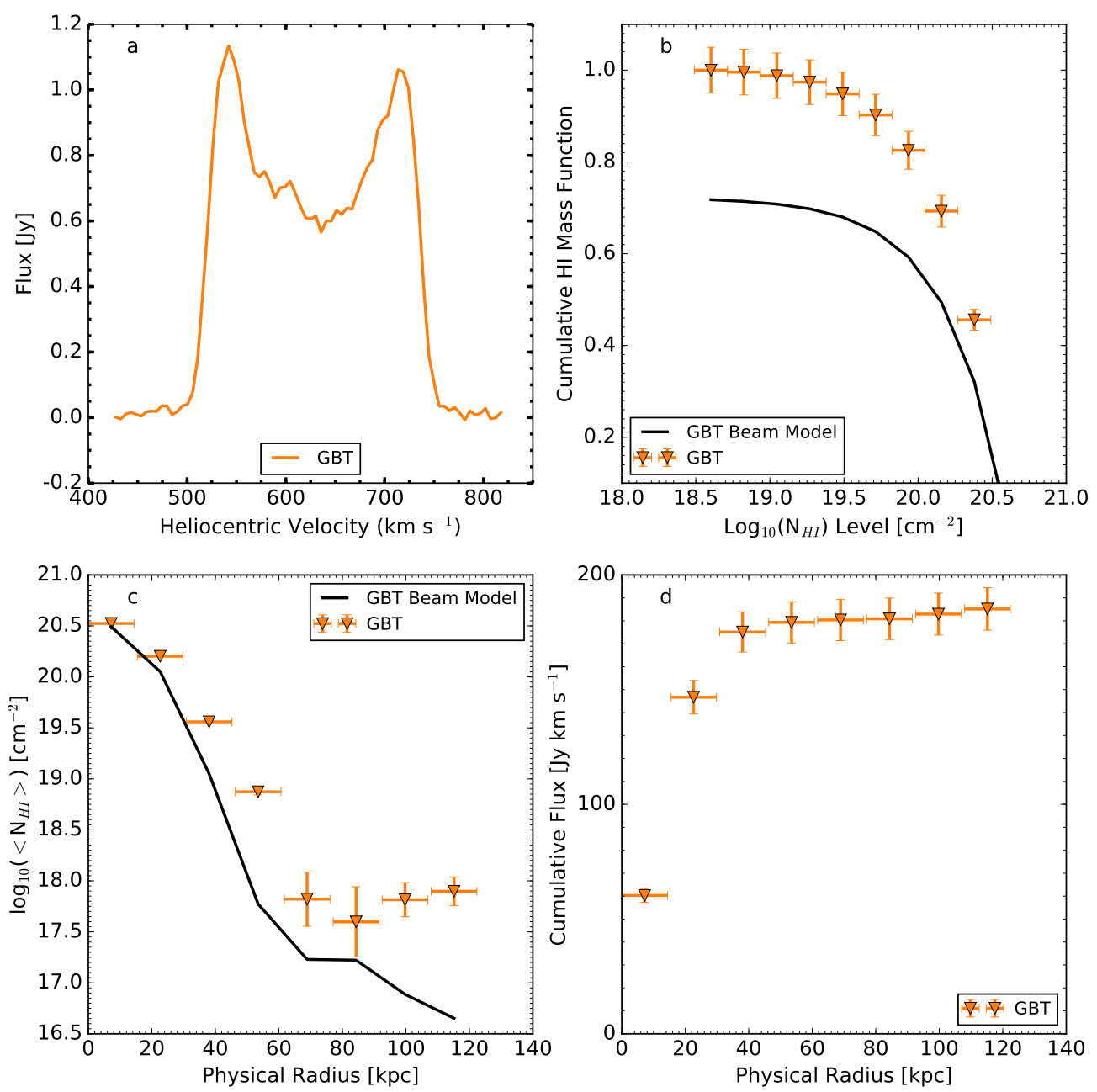

Figure 3.26 Summary of the various properties derived from the regridded GBT (orange inverted triangles) data set of NGC1003. The panel descriptions are the same as described in the caption for Figure 3.25. 
UGC4278
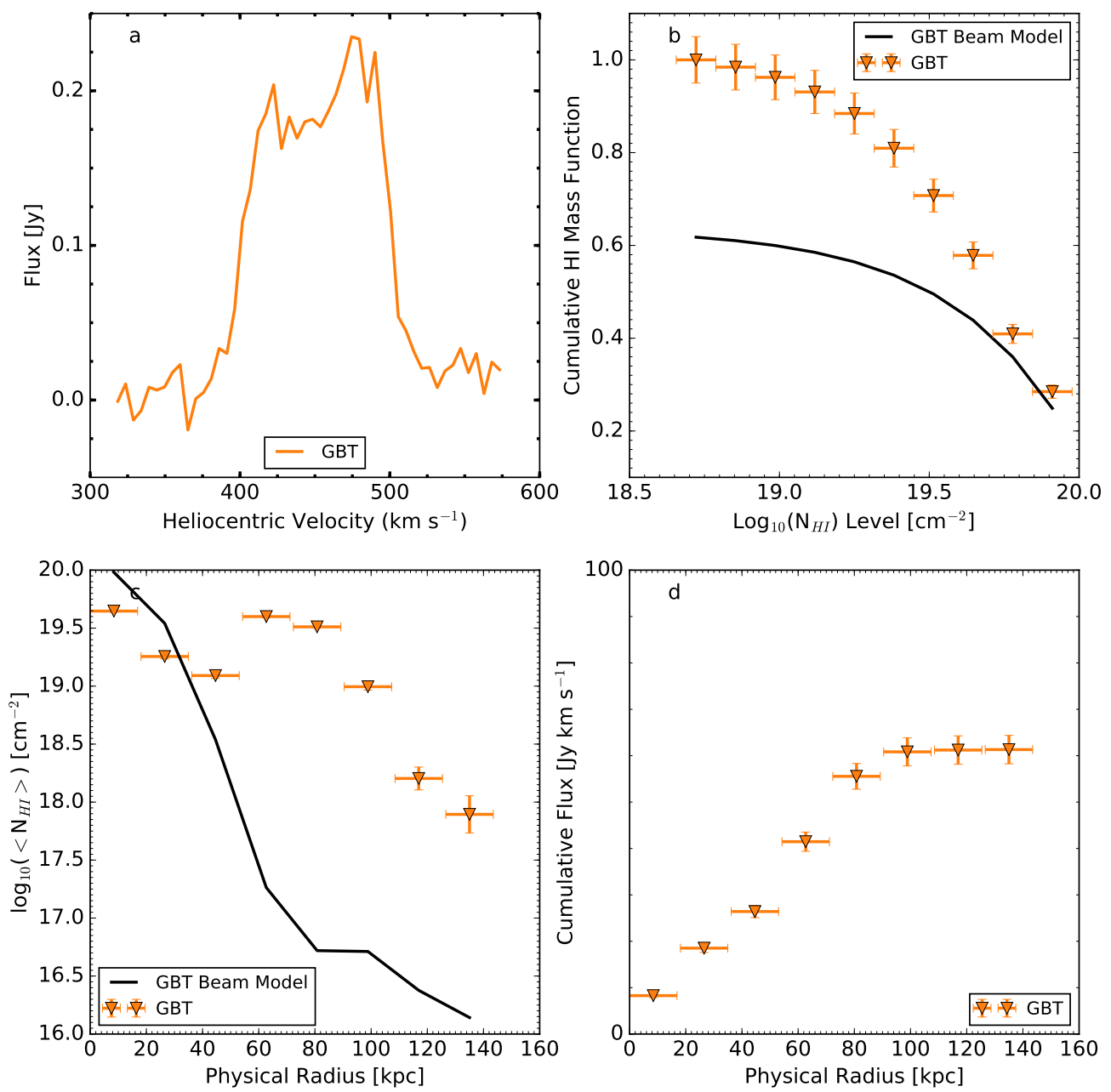

Figure 3.27 Summary of the various properties derived from the regridded GBT (orange inverted triangles) data set of UGC4278. The panel descriptions are the same as described in the caption for Figure 3.25. 
NGC2541
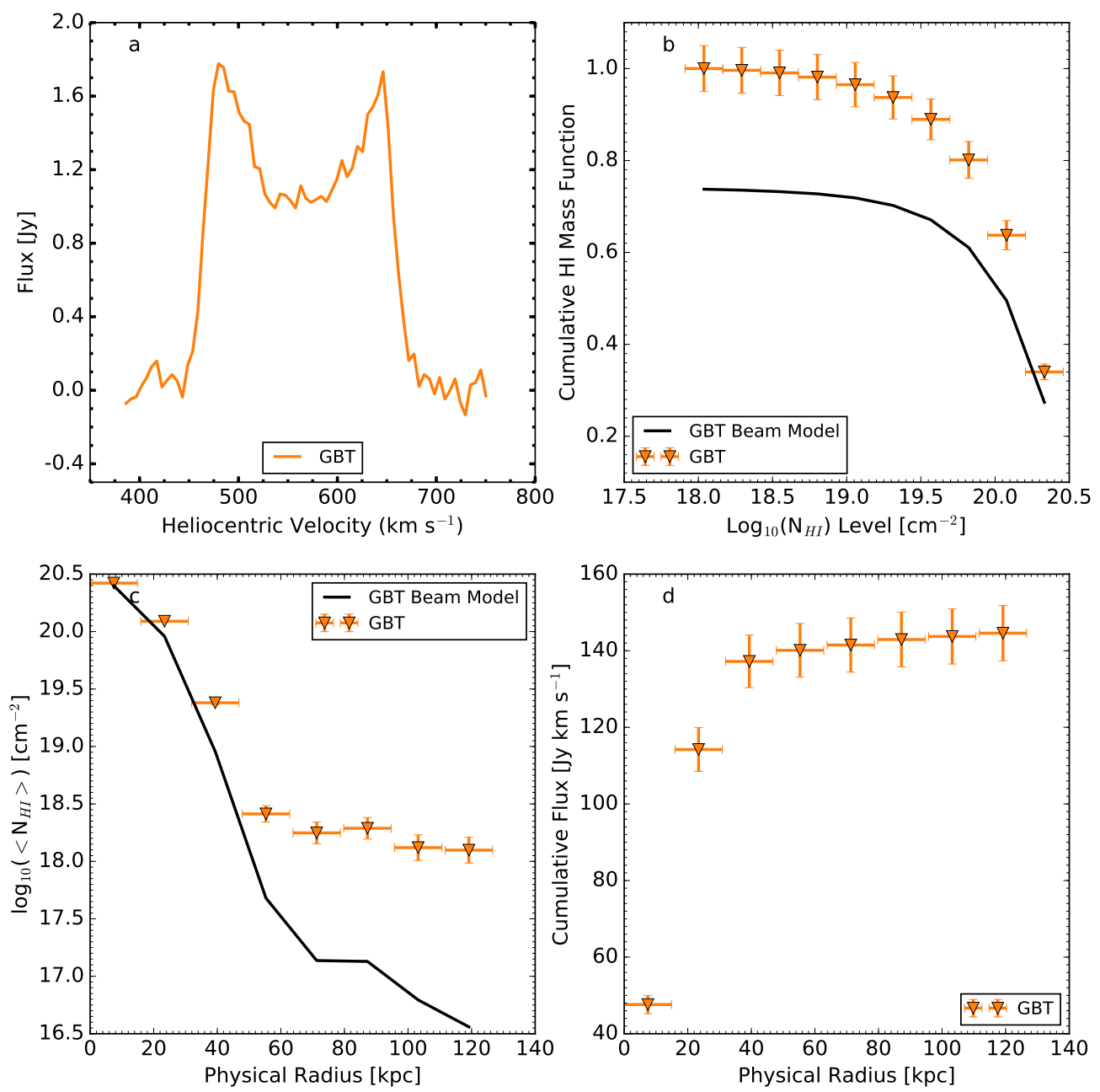

Figure 3.28 Summary of the various properties derived from the regridded GBT (orange inverted triangles) data set of NGC2541. The panel descriptions are the same as described in the caption for Figure 3.25. 

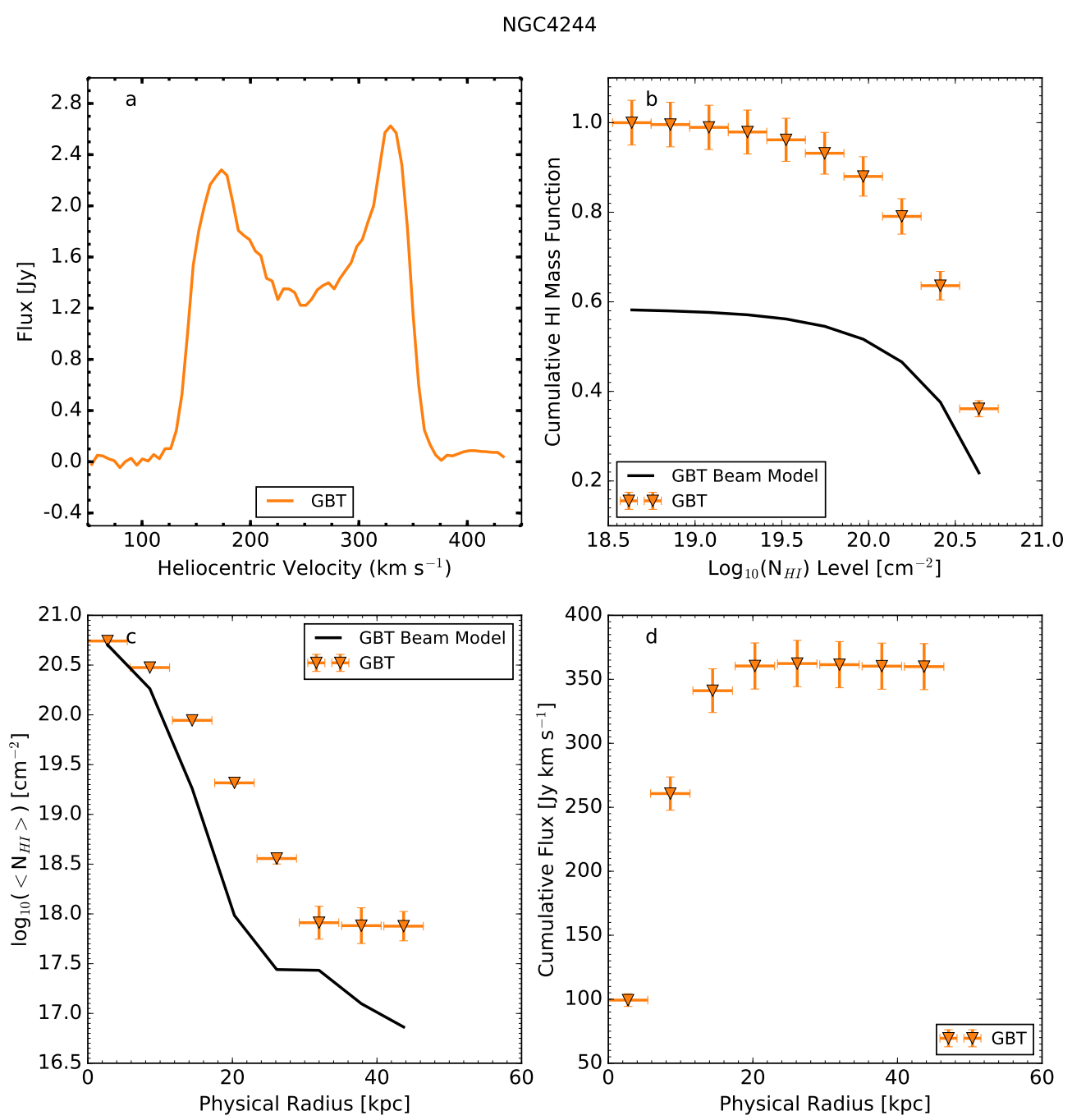

Figure 3.29 Summary of the various properties derived from the regridded GBT (orange inverted triangles) data set of NGC4244. The panel descriptions are the same as described in the caption for Figure 3.25. 
NGC4258
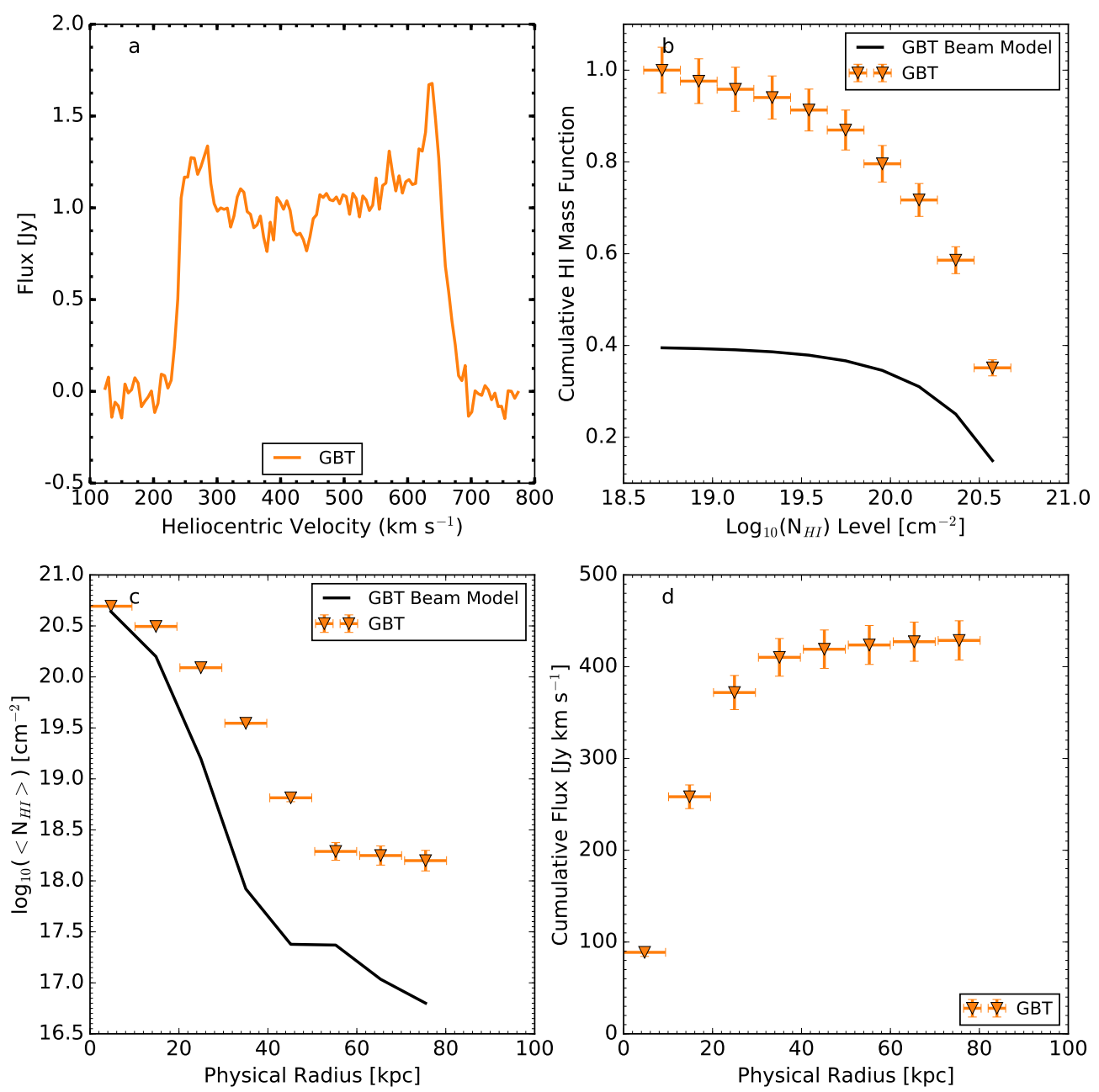

Figure 3.30 Summary of the various properties derived from the regridded GBT (orange inverted triangles) data set of NGC4258. The panel descriptions are the same as described in the caption for Figure 3.25. 
NGC4559
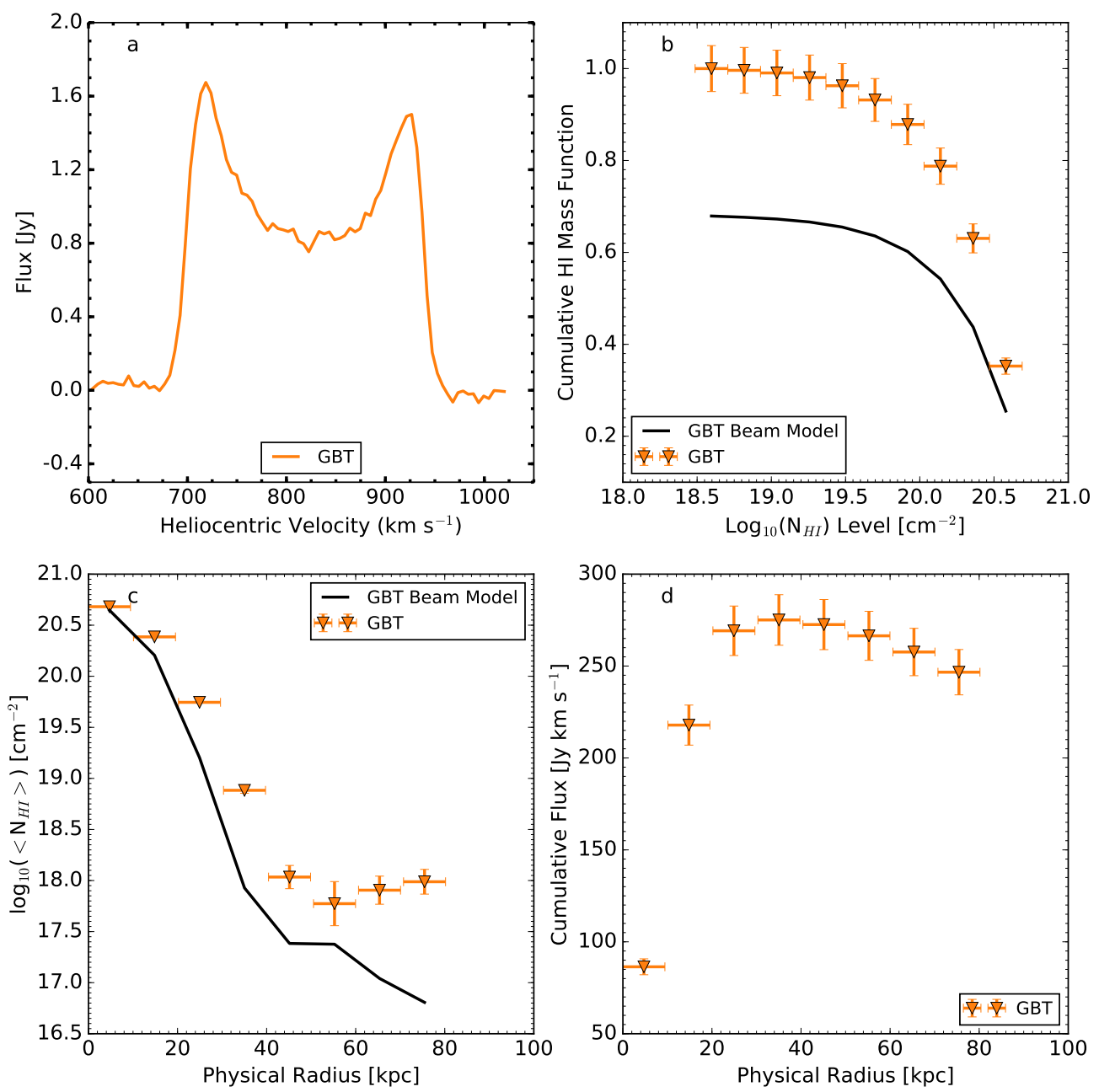

Figure 3.31 Summary of the various properties derived from the regridded GBT (orange inverted triangles) data set of NGC4559. The panel descriptions are the same as described in the caption for Figure 3.25. 
UGC7774
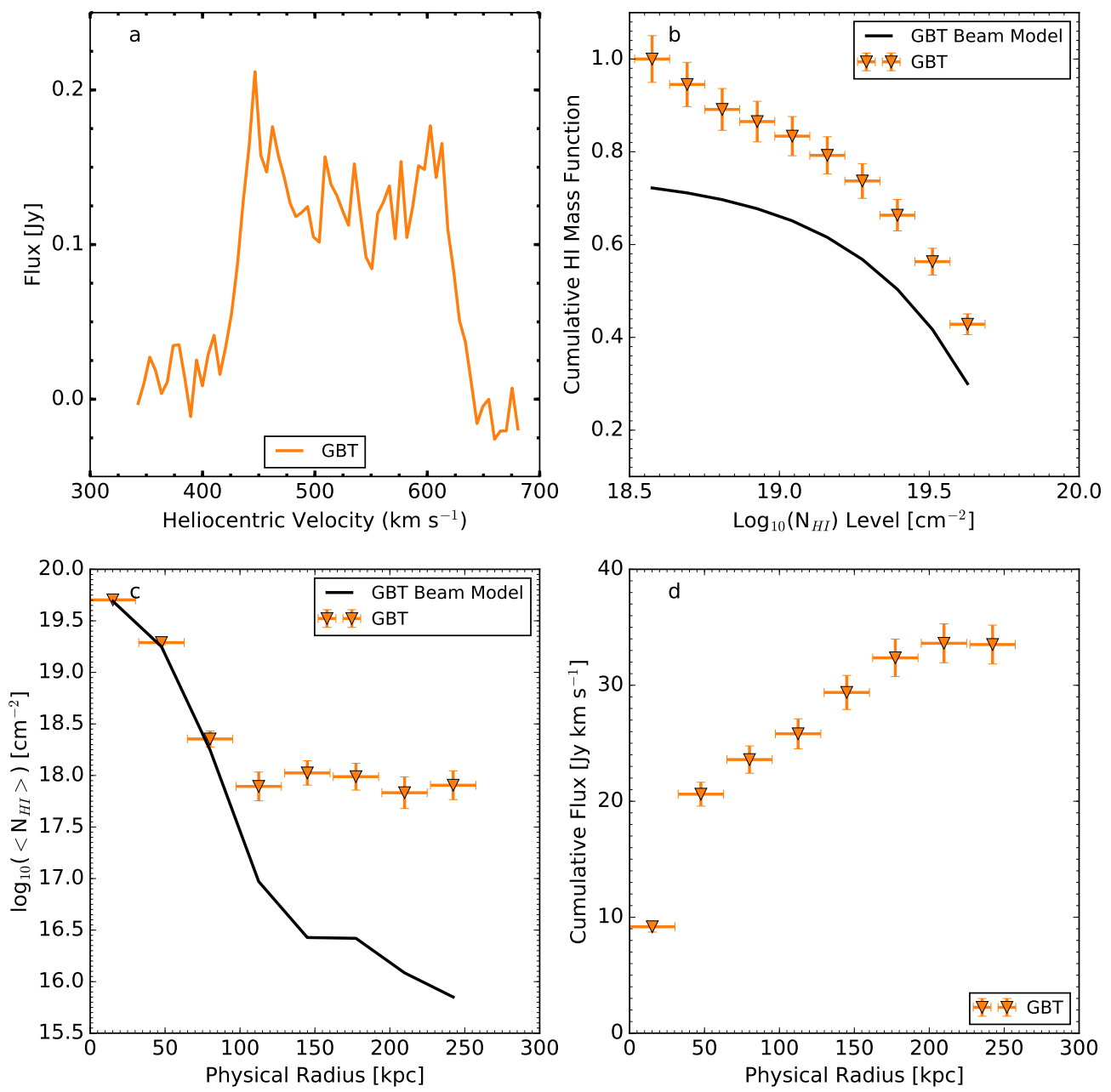

Figure 3.32 Summary of the various properties derived from the regridded GBT (orange inverted triangles) data set of UGC7774. The panel descriptions are the same as described in the caption for Figure 3.25. 
NGC4631
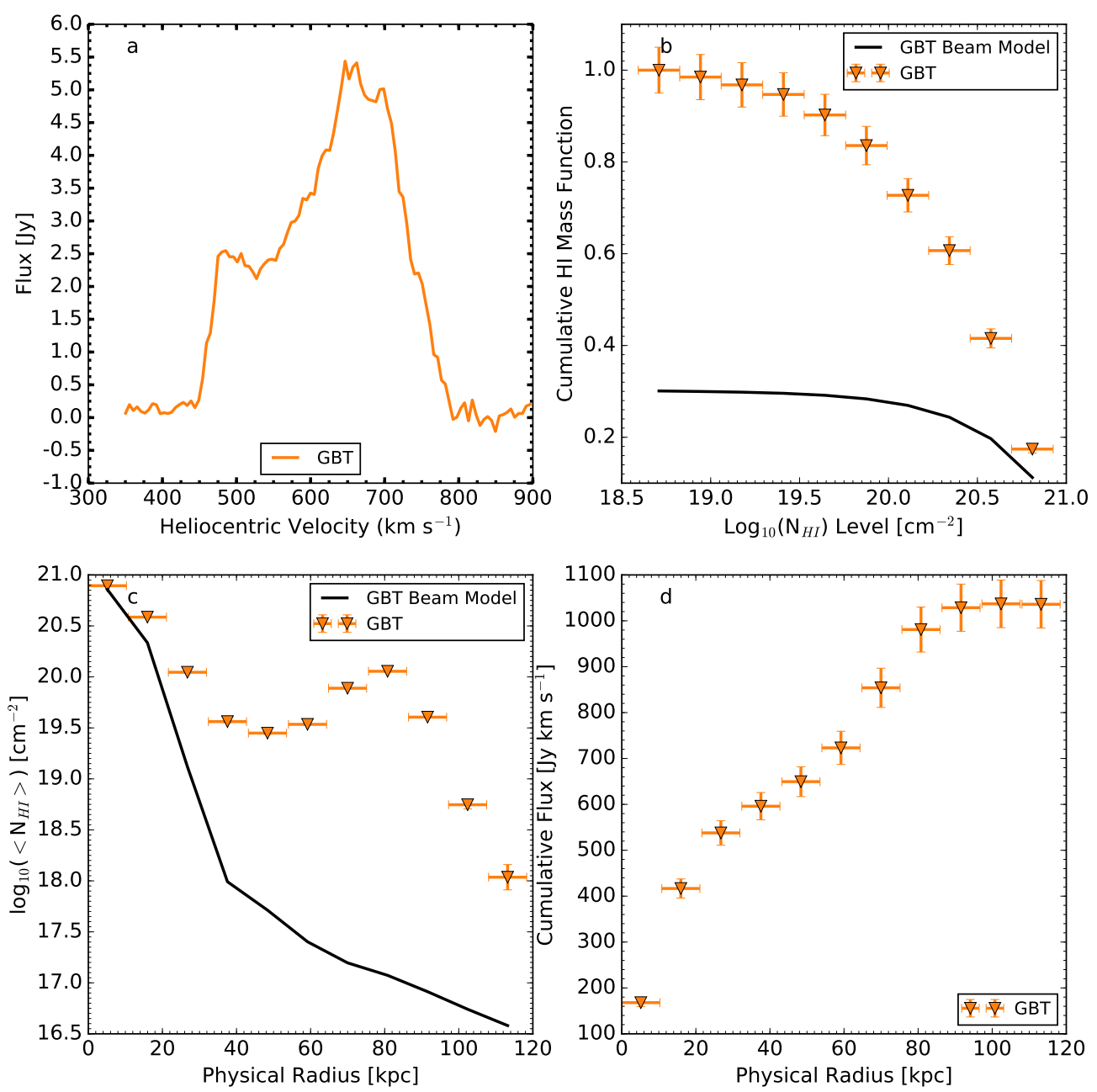

Figure 3.33 Summary of the various properties derived from the regridded GBT (orange inverted triangles) data set of NGC4631 The panel descriptions are the same as described in the caption for Figure 3.25. 
NGC5023
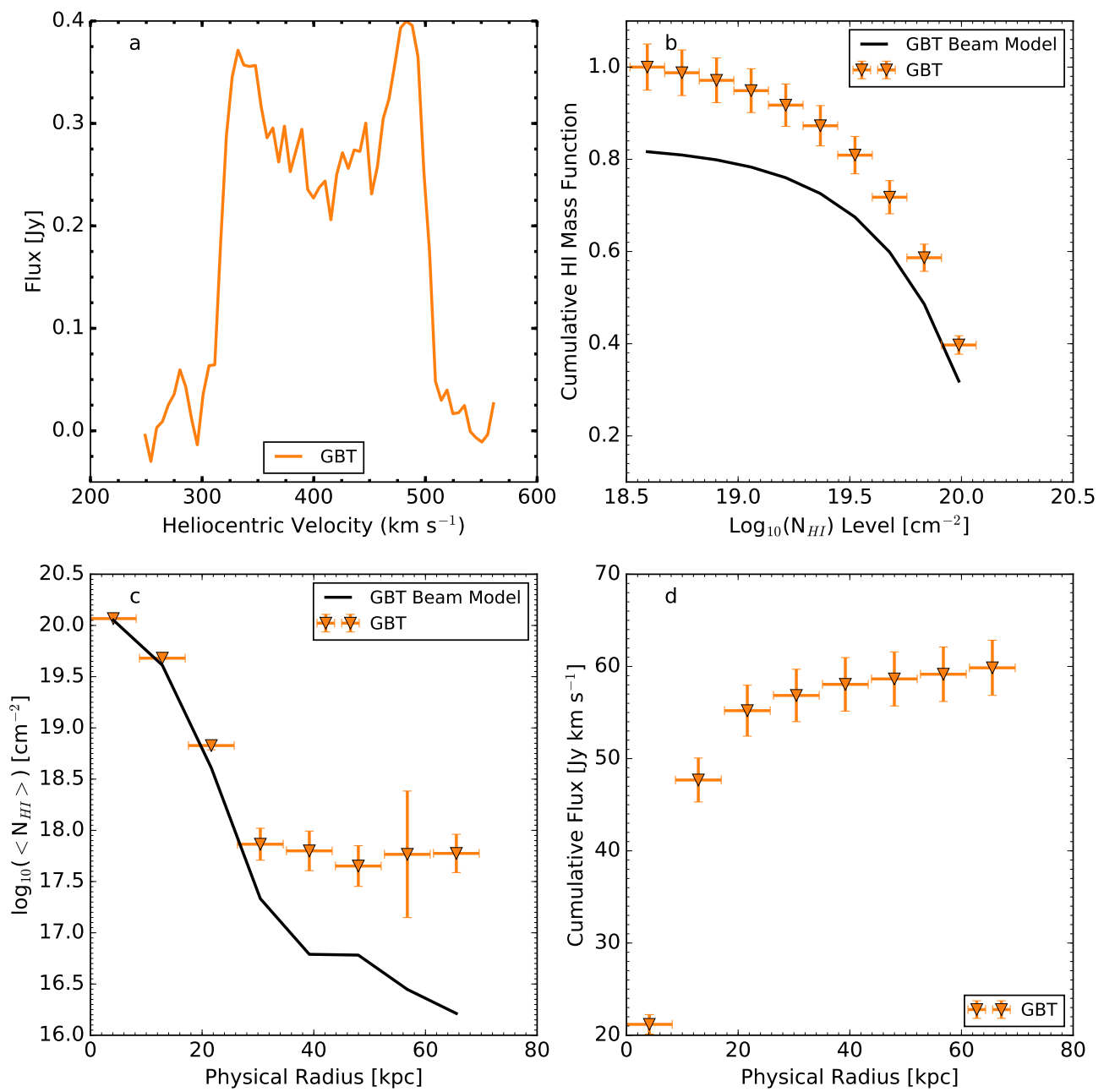

Figure 3.34 Summary of the various properties derived from the regridded GBT (orange inverted triangles) data set of NGC5023. The panel descriptions are the same as described in the caption for Figure 3.25. 

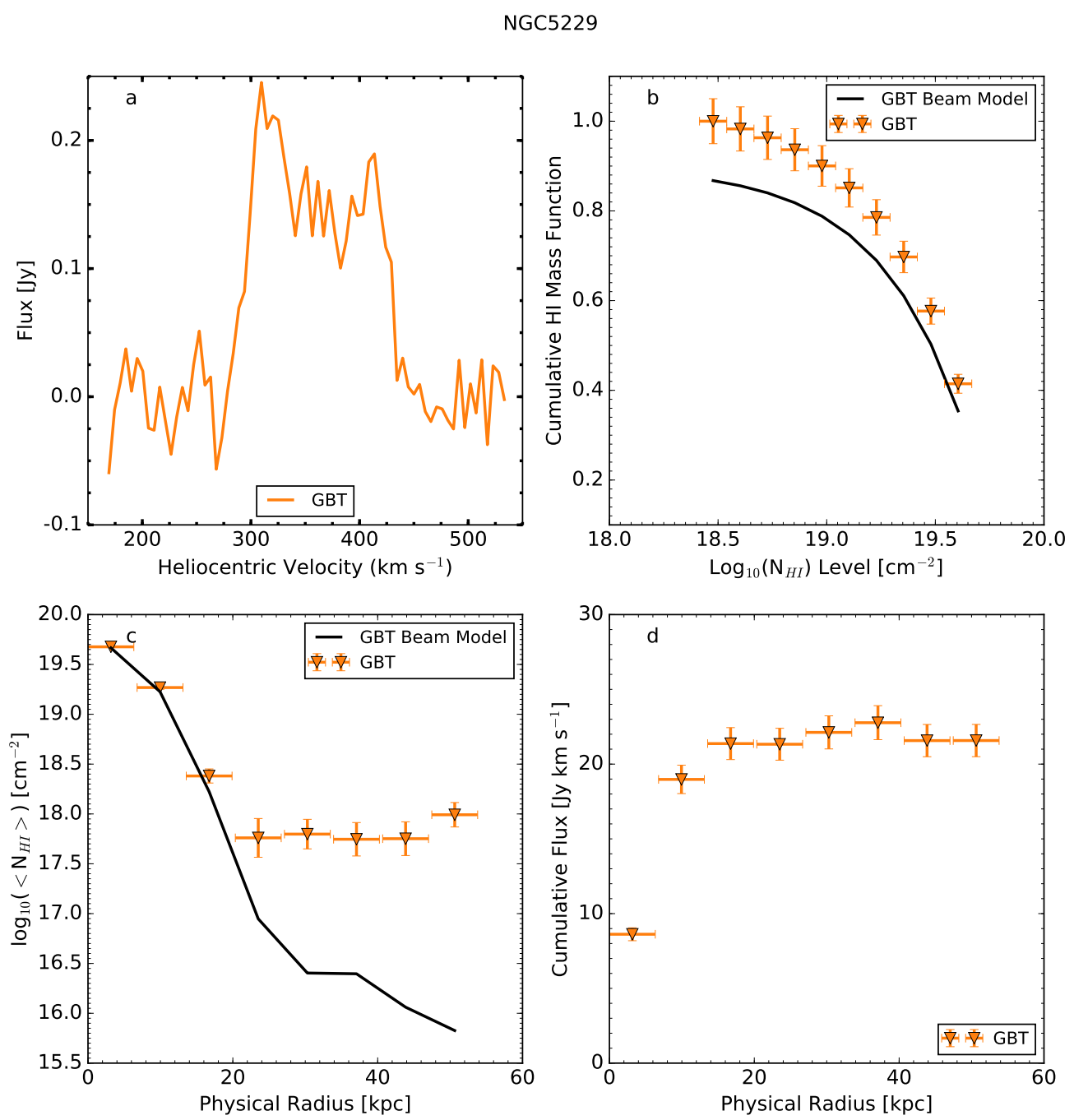

Figure 3.35 Summary of the various properties derived from the regridded GBT (orange inverted triangles) data set of NGC5229. The panel descriptions are the same as described in the caption for Figure 3.25. 
NGC5585
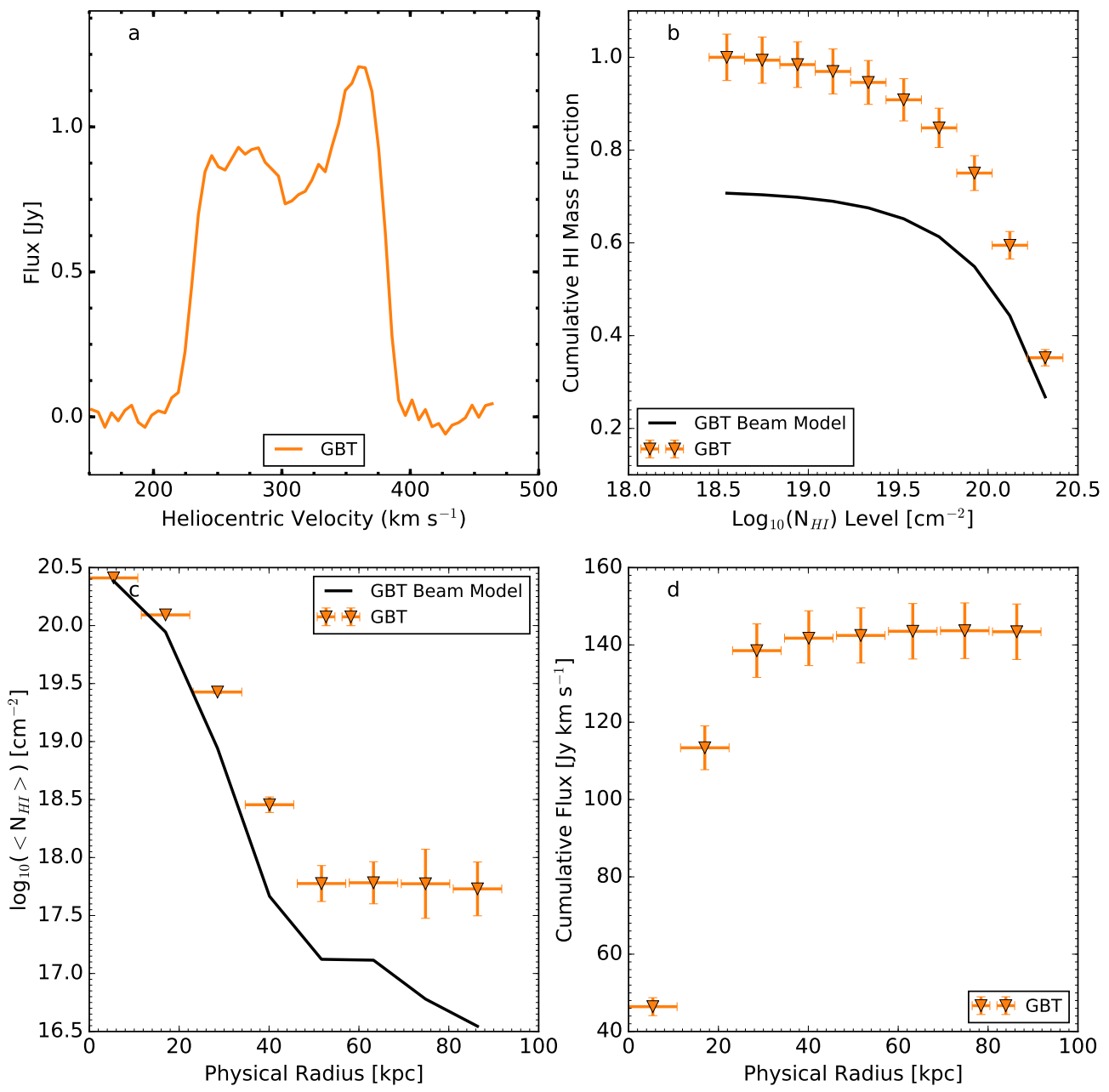

Figure 3.36 Summary of the various properties derived from the regridded GBT (orange inverted triangles) data set of NGC5585. The panel descriptions are the same as described in the caption for Figure 3.25. 
Table 3.3 Summary of Derived Properties

\begin{tabular}{lccccccc}
\hline \hline Source & GBT Hi Mass $\left[10^{9} M_{\odot}\right]$ & $\mathrm{M}_{*}{ }^{\mathrm{a}}\left[10^{10} M_{\odot}\right]$ & $\mathrm{M}_{\text {bary }} \mathrm{b}\left[\times 10^{10} M_{\odot}\right]$ & Deprojected Physical Area $\left[\mathrm{kpc}^{2}\right]$ & $\mathrm{V}_{\text {rot }} \mathrm{c}$ & $\mathrm{GBT}_{19}$ & $\rho^{\mathrm{d}}$ \\
\hline NGC672 & $3.45 \pm 0.17$ & $3.12 \pm 0.30$ & $7.81 \pm 0.36$ & $201 \pm 1$ & $98 \pm 3$ & $0.013 \pm 0.007$ & 0.16 \\
NGC1003 & $5.75 \pm 0.29$ & $2.62 \pm 0.27$ & $10.44 \pm 0.39$ & $108 \pm 1$ & $94 \pm 2$ & $0.022 \pm 0.002$ & 0.54 \\
UGC4278 & $0.95 \pm 0.05$ & $0.61 \pm 0.07$ & $1.90 \pm 0.08$ & $98 \pm 1$ & $77 \pm 2$ & $0.098 \pm 0.005$ & 0.24 \\
NGC2541 & $8.99 \pm 0.45$ & $4.75 \pm 0.50$ & $16.98 \pm 0.65$ & $91 \pm 1$ & $85 \pm 2$ & $0.098 \pm 0.005$ & 0.25 \\
NGC4244 & $1.74 \pm 0.09$ & $1.87 \pm 0.20$ & $4.24 \pm 0.21$ & $324 \pm 1$ & $89 \pm 2$ & $0.014 \pm 0.001$ & 0.39 \\
NGC4258 & $6.22 \pm 0.31$ & $47 \pm 5$ & $56 \pm 5$ & $1057 \pm 1$ & $198 \pm 5$ & $0.065 \pm 0.003$ & 0.57 \\
NGC4559 & $4.22 \pm 0.02$ & $9.59 \pm 0.96$ & $15.03 \pm 0.96$ & $455 \pm 1$ & $103 \pm 2$ & $0.016 \pm 0.001$ & 1.01 \\
UGC7774 & $3.93 \pm 0.02$ & $0.12 \pm 0.01$ & $5.47 \pm 0.20$ & $6014 \pm 2$ & $80 \pm 3$ & $0.300 \pm 0.002$ & 0.59 \\
NGC4631 & $14.29 \pm 0.71$ & $0.059 \pm 0.006$ & $25.31 \pm 0.92$ & $100 \pm 1$ & $138 \pm 3$ & $0.042 \pm 0.002$ & 0.41 \\
NGC5023 & $0.60 \pm 0.03$ & $0.85 \pm 0.05$ & $1.66 \pm 0.09$ & $80 \pm 2$ & $0.098 \pm 0.005$ & 0.36 \\
NGC5229 & $0.14 \pm 0.01$ & $0.32 \pm 0.03$ & $0.51 \pm 0.03$ & $9.3 \pm 0.1$ & $55 \pm 2$ & $0.220 \pm 0.002$ & 0.33 \\
NGC5585 & $2.06 \pm 0.10$ & $2.00 \pm 0.03$ & $4.80 \pm 0.22$ & $63 \pm 1$ & $0.035 \pm 0.002$ & 0.17 \\
\hline
\end{tabular}

\author{
${ }^{a}$ Stellar Mass \\ ${ }^{b}$ Baryonic Mass \\ ${ }^{c}$ Rotation velocity taken from Hyperleda search \\ ${ }^{d}$ Galaxy Density from Tully (1988)
}

\title{
3.4.2 The Diffuse Hi Mass Fraction
}

In the previous chapter, $f_{19}$ was plotted verses several inherent galaxy properties that, according to simulations, are associated with cold mode accretion. This analysis applied to the full sample is shown in Figure 3.37.

Overall, there is no explicit trend between $f_{19}$ and any of the galaxy properties. The lack of a trend with projected Hi angular area again ensures this analysis is not resolution limited. If such a bias existed, the highest $f_{19}$ values would be associated with the smallest projected areas due to the unphysical $N_{\mathrm{HI}}$ values caused by unresolved emission distribution. Interestingly, a noticeably fraction of galaxies fall below the baryonic mass threshold from Kereš et al. (2005) and have relatively high $f_{19}$ values. Likewise, an appreciable number of sources with larger $f_{19}$ values fall below the the threshold for gas dominated galaxies set by Kannappan et al. (2013). That said, the scatter in $f_{19}$ for galaxies below this threshold is quite large. It is 
nevertheless possible that the high $f_{19}$ values that fall below the predicted thresholds for cold mode accretion have distinct underlying origins related to other measured galaxy properties. For example, high $f_{19}$ values below the mass threshold with some positive correlation to high galaxy density may signify the excess diffuse Hi is due to tidal interactions. To test for a potential underlying origin associated with the other galaxy properties, the two sample Kolmogorov-Smirnov (KS) statistic is computed between the distributions that lie above and below the baryonic mass and rotation velocity threshold for the measured quantities of $\rho$, sSFR, and $\Sigma_{\mathrm{SFR}}$. The two sample KS-test in this context will determine whether the two distinct samples that fall above and below the aforementioned thresholds could be drawn from the same parent cumulative distribution of the measured parameters of $\rho$, sSFR, and $\Sigma_{\mathrm{SFR}}$. If the null hypothesis can be rejected for any of these measured quantities, it indicates the differences in the two galaxy samples segregated by the mass and rotational velocity may originate from some physical characteristic of these galaxies (potentially related to the existence of cold flows) rather than the inherent spread of the measured parameters. In all cases, however, the returned p-value is greater than 0.5, demonstrating that the null hypothesis cannot be rejected; thus, the differences between the two galaxy samples above and below the mass and rotational velocity thresholds are most likely due to the inherent spread of — for example, the $\Sigma_{\mathrm{SFR}}$ values - rather than an underlying relation to the galaxy properties.

A final interesting aspect of Figure 3.37 is that higher $f_{19}$ are associated over a large range of indicators for star formation activity. In fact, most of the highest $f_{19}$ values relate to the lowest star formation surface densities, suggesting that outflows 
do not play a large role in the production of the excess diffuse Hi observed with the GBT.

The lack of discernible trends, and considering that the median $f_{19}$ of the included galaxies is 0.02 , suggests that the nearby galaxies in the HALOGAS sample do not harbor significant low column density gas reservoirs that are both neutral and extended.

\subsection{The Dearth of Diffuse Hi}

The previous chapter ended with a discussion on what type of observational constraints would need to be present between $f_{19}$ and the galaxy properties explored in Figure 3.37. Namely, a comparatively high $f_{19}$ value would need to be correlated with a small $\rho$ indicating lack of tidal perturbations, fall below the predicted thresholds for $M_{\text {bary }}$ and $V_{\text {rot }}$. Taking the three highest $f_{19}$ values, which are $0.30 \pm 0.02$, 0.22 \pm 0.02 , and $0.16 \pm 0.01$ for UGC7774, NGC5229, and NGC4414, respectively, only NGC5229 falls within all necessary constraints.

Deep high-resolution Hi observations have revealed extraplanar gas components consisting of low column density gas $\left(N_{H I} \sim 10^{19} \mathrm{~cm}^{-2}\right.$ that make an appreciable fraction of the total Hi mass (e.g., Fraternali et al. 2002; Oosterloo et al. 2007; Boomsma et al. 2008) and also contribute significantly to the full kinematic picture through vertical velocity gradients. It is therefore somewhat surprising to not detect diffuse Hi around galaxies with known substantial extraplanar components. While GBT observations presented in de Blok et al. (2014a) and Pisano (2014) have re- 

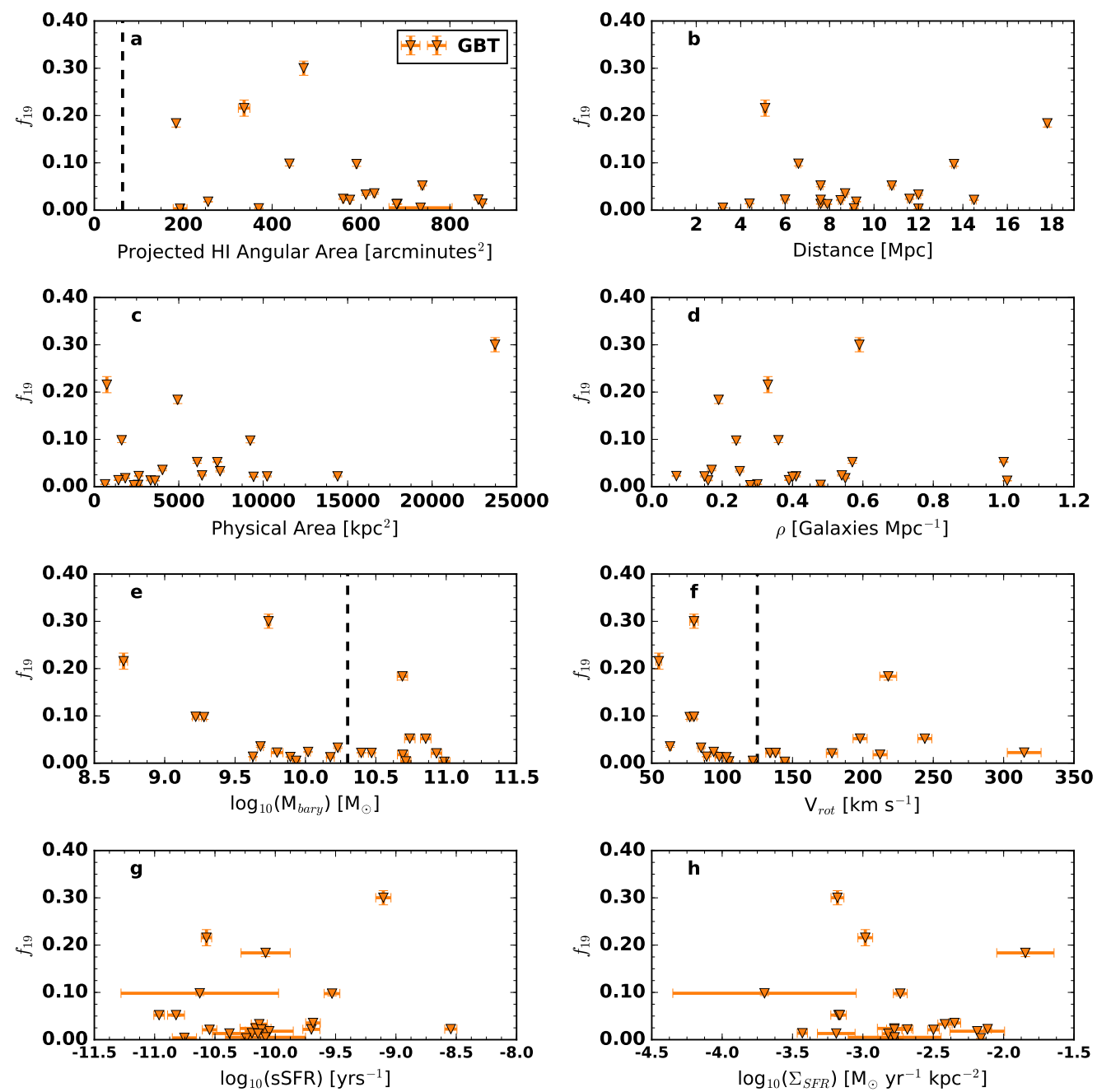

Figure 3.37 Environmental and intrinsic property relations with the diffuse Hi mass ratio, $f_{19}$. a: $f_{19}$ as a function of projected angular area. The vertical dashed line marks the area of the GBT beam (up to the FWHM); b: $f_{19}$ as a function of distance; c: $f_{19}$ as a function of projected physical area; $\mathrm{d}: f_{19}$ as a function of galaxy density. $\rho$ is taken from Tully (1988); e: $f_{19}$ as a function of the source galaxy baryonic mass $\left(\mathrm{M}_{H I}+\mathrm{M}_{*}\right)$. The vertical dashed line corresponds to the baryonic mass threshold from Kereš et al. (2005) below which cold mode accretion is the dominant accretion mechanism; f: $f_{19}$ as a function of rotational velocity. The vertical dashed line corresponds to the threshold mass at which galaxies should be gas dominated (Kannappan et al., 2013); g: $f_{19}$ as a function of specific SFR (sSFR); h: $f_{19}$ as a function of the surface density of star formation $\left(\Sigma_{S F R}\right)$. 
vealed diffuse Hi around nearby galaxies, the origin of the features remain unclear. The analysis presented in this and the preceding chapter, which included the sources studied in those works, did not reveal them to be distinct from other well-resolved sources.

\subsection{Conclusions}

The analysis presented in this work of the GBT observations of the nearby galaxies of the HALOGAS sample has not revealed significant diffuse Hi features. The dearth of significant Hi reservoirs - even in the galaxies predicted by cosmological simulations to potentially harbor cold flows - has two major implications for current and planned large scale Hi surveys:

- If one wishes to detect the low column density Hi that potentially exists at the interface between the disk and the CGM, future facilities must invest the necessary telescope time to reach $N_{\mathrm{HI}}$ sensitives on the order of $10^{17} \mathrm{~cm}^{-2}$

- Future surveys should consider targeted observations of a clumpy medium given the absence of a significant extended emission around these galaxies.

As has been discussed, sources have been observed in emission at $N_{H I} \sim 10^{17}$ $\mathrm{cm}^{-2}$ (Wolfe et al., 2013) - albeit in the form of a clumpy medium consisting of discrete clouds only a few kpc across. While the origin of these clouds is also remains an unresolved question, it is evidence towards the existence of the diffuse HI predicted by cosmological simulations (e.g., Popping et al. 2009; Nuza et al. 2014). The photoioniztion models of Bland-Hawthorn et al. (2017), which were 
modified to consider the clumpiness of the outer disk regions of a typical galaxy, show the Hi can survive in an external radiation field to extents of $\sim 50-70 \mathrm{kpc}$ with only a moderate filling factor, consistent with similar GBT observations that were presented here. If detected in high-resolution observations, such data would provide incredible insights into the kinematics of extended disk structure and probe detailed properties of the ionization interface caused by extragalactic radiation fields. 


\title{
Chapter 4
}

\section{Neutral Hydrogen Observations with the Focal L-Band Array for the Green Bank Telescope (FLAG) ${ }^{1}$}

\begin{abstract}
A description of the system, observing procedure, data reduction, and preliminary results from commissioning of the Focal L-Band Array for the Green Bank Telescope (FLAG) — a cryogenically cooled 19 dual-polarization phased array feed (PAF) — is presented. Specifically, work related to the commissioning of the HI spectral line mode is presented. Extragalactic HI observed with FLAG compares well with equivalent data taken with the existing GBT single-pixel L-Band feed. The necessary survey time for FLAG to reach an equivalent rms noise levels when compared to similar single-pixel maps is reduced by a factor of five to seven.
\end{abstract}

\subsection{Introduction}

The increased field-of-view (FOV) provided by phased array feeds (PAFs), and the subsequent increase in survey speed, constitutes the next major advancement in radio astronomy instrumentation. Such arrays have been used commercially for decades (Jeffs et al., 2008), but the unique challenge of operating at extremely low

\footnotetext{
${ }^{1}$ I wish to acknowledge D. J. Pisano, R. Prestage, R. Black, B. Jeffs, M. Ruzindana, M. Burnett, K. Warnick, and A. Roshi as collaborators on this work presented in this chapter.
} 
signal intensities inherent to the detection of astrophysical signals has only recently been overcome (Vilnrotter et al., 1995). Placing densely packed dipole antennas in the focal plane of a radio telescope allows for increased sampling of the incident plane waves of faint astronomical signals. Correlating the generated raw complex voltages and then multiplying by complex coefficients (i.e., beamforming weights) will illuminate the aperture in a manner such that the resulting sky power patterns mimics a multi-beam feed ${ }^{1}$ (e.g. Landon et al. 2010; Fisher \& Bradley 2000), while avoiding the engineering challenges of positioning physically distinct feeds. This is an especially powerful shortcut for low frequency observations where large physical feeds are necessary that sparsely sample a limited fraction of sky at one instant.

Several PAFs have already been deployed on both large single dishes, such as the $64 \mathrm{~m}$ Parkes single dish and aperture synthesis arrays with very encouraging science results. For instance, Reynolds et al. (2017) successfully recreated a detailed neutral hydrogen $(\mathrm{HI})$ column density $\left(N_{\mathrm{HI}}\right)$ map of the Large Magellanic Cloud originally observed with the Parkes 21cm Multi-beam Receiver, as well as direct Hi detections of source from the Hi Parkes All-Sky Survey (HIPASS; Barnes et al. 2001) and hydrogen recombination lines. Prototype PAFs for the CSIRO Australian SKA Pathfinder (ASKAP; Heywood et al. 2016) and the nearly APERTIF upgrade to the Westerbork Radio Telescope (WSRT; Oosterloo et al. 2009) have shown excellent capability for wide-field imaging, which will dramatically cut survey speeds.

While the increase in the FOV will be immensely useful on array telescopes

\footnotetext{
${ }^{1} \mathrm{~A}$ traditional multi-beam receiver packs several distinct feed horns into the focal plane of the telescope.
} 
like the WSRT or ASKAP, such instruments will still suffer from the inherent limitation of missing data in the center of the $u v$-plane. The missing short baselines reduce the surface brightness sensitivity by limiting the angular scales over which the instrument is sensitive (Braun \& Walterbos, 1985). Single dish observations that contain the missing data for the low spatial frequencies may still be necessary even in the age of the SKA and its precursor array telescopes. The unblocked aperture of the 100m Green Bank Telescope (GBT) reduces the pickup of stray radiation leading to uniform illumination across the primary reflector, meaning potentially unprecedentedly highly sensitive electronically formed beams of a PAF, while also possessing relatively good angular resolution. The potential to decrease the telescope time required for deep $\left(N_{\mathrm{HI}} \leq 10^{18} \mathrm{~cm}^{-2}\right)$ on-the-fly HI mapping of extended sources Hi will make a PAF equipped GBT the ideal instrument to look for previously undetected diffuse Hi around nearby galaxies related to potential cold flows (Kereš et al., 2005, 2009).

The Focal L-Band Array for the Green Bank Telescope (FLAG) is a 19 element, dual polarization PAF with cryogenically cooled low noise amplifiers (LNAs) that maximize sensitivity. Previous commissioning observations of this PAF have shown excellent performance in terms of sensitivity and spectral line imaging capabilities (Roshi et al., 2018). In fact, as of Spring 2018, FLAG holds the current world record for the lowest reported system temperature $\left(T_{\text {sys }}\right)$ normalized by aperture efficiency $\eta$ at $25 \pm 3 \mathrm{~K}$ near $1350 \mathrm{MHz}$ for a central beam that is electronically formed (Roshi et al., 2018). This value is on par with the capabilities of the existing single-pixel L-Band receiver. This chapter describes aspects of a new beamforming 
backend and discusses the first research quality results and current status of the system. Specifically, the important questions about the system that are explored in this chapter are: over what time scales do the complex beamformer weights begin to degrade? Given similar performance of the two receivers, how well do similar mapping observations explicitly compare between FLAG and the single-pixel feed? What are the comprehensive noise properties of all electronically formed beams and on what time scales (if any) do the noise properties fluctuate? Furthermore, the observations with the PAF discussed in Roshi et al. (2018) were performed with a relatively narrow bandwidth and different polyphase filterbank (PFB) implementation for fine channelization. The focus of this chapter is on FLAG spectral line mapping observations over a variety of extragalactic sources that will clarify some of the remaining questions on the capability of FLAG and the new beamformer that is now in place.

Section 4.2 delves into the theoretical framework of beamforming and the subsequent derivation and application of complex beamformer weights (specifically those that maximize the signal-to-noise); Section 4.3 discusses the specific details of the new production backend; Section 4.4 highlights the specific properties of the calibration and science observations before discussing the custom software package utilized in the calibration and imaging of the these data; Section 1.5 shows the results and examines outstanding questions on performance the performance of FLAG; and Section 1.6 discusses what these latest results mean in the context of the status and future of FLAG as a publicly available instrument on the GBT and its potential scientific contributions. 


\subsection{Theory of Optimal Beamforming}

,

As alluded to Section 1.5, the process of 'beamforming' involves the weighted sum of the individual sensor responses to an incident astronomical signal. However, before discussing specific examples and the scientific applications of beamforming, we must first introduce an additional term, $\mathbf{n}(t)$, that encapsulates the inherent noise of the system caused, in part, by spillover noise (i.e. the warm ground as seen from the individual sensors (henceforth 'dipoles'), sky noise from atmospheric and cosmic background radiation, and receiver noise from the front end amplifiers. Such noise can be approximated by an additive zero-mean, time-dependent Gaussian stochastic process. The vector $n(t)$ is shaped $M \times 1$ and characterizes the noise in an $M$ element PAF. The signal model is now of the form as introduced in Landon et al. (2010)

$$
\mathbf{z}(t)=\mathbf{a}(\theta) \mathbf{s}(t)+\mathbf{n}(t)
$$

where $s(t)$ is also a $M \times 1$ vector that characterizes the array response (i.e., the recorded complex voltages) in the direction of $\theta$ to an incident signal. In this application, $\mathbf{a}(\theta)$ is the $M \times 1$ array response vector. A dipole response can be multiplied by any complex value, $w_{\mathrm{i}}$, in order to change the estimate of $z_{\mathrm{i}}(t)$. Extending this to all dipole responses, a new estimate of $z(t)$ can be found for any particular $\theta$ by computing a weighted sum of the dipole responses

$$
\hat{\mathbf{z}}(t)=\sum_{i=1}^{\mathrm{M}} \mathbf{w}_{\mathrm{i}}^{*} \mathbf{z}(t)=\mathbf{w}^{\mathrm{H}} \mathbf{z}(t) .
$$


Here, w is called the beamforming weight vector that holds the weight for each individual dipole, which effectively alters the aperture illumination pattern seen by that dipole. The $H$ subscript denotes the Hermition (complex conjugate transpose) form of the vector. The process of choosing $\mathbf{w}$ to obtain $\hat{z}(t)$ is known as 'beam steering'.

In radio astronomy, where the signals are inherently extremely faint, it is advantageous for an observer to compute weights that maximize the signal-to-noise from a given detection. The individual dipole responses measured over a discrete time sample (i.e., integration) can conveniently be arranged in a covariance matrix

$$
\mathbf{R}=\mathbf{z}^{\mathbf{H}}(\mathbf{t}) \mathbf{z}(\mathbf{t})
$$

where $\mathbf{R}$ is now an $M \times M$ matrix of complex values that characterizes the correlations between the recorded complex voltages of the individual dipole elements. Jeffs et al. (2008) goes on to characterize the signal from the array by the equation

$$
\mathbf{R}=\mathbf{R}_{\mathrm{s}}+\mathbf{R}_{\mathrm{n}}
$$

where $\mathbf{R}_{\mathrm{s}}=\sigma^{2} \mathbf{a}(\theta) \mathbf{a}^{\mathbf{H}}(\theta)$ is the signal covariance matrix and $\mathbf{R}_{\mathrm{n}}$ contains the noise covariance from spillover, background, and the mutual coupling of the dipoles (where a dipole picks up the thermal signatures of the adjacent dipoles).

The noise covariance can be measured by pointing the telescope to a blank patch of sky so that $\mathbf{z}(\mathbf{t}) \approx \mathbf{n}(\mathbf{t})$. Equation 4.1 is obtained by pointing at a strong 
( $>10 \mathrm{Jy}$ ) point source. In this context, $\mathbf{a}(\theta)$ is the direction vector in the direction, $\Omega$. Compute Equation 4.4 for each signal-noise pair of covariance matrices and sum to obtain $\mathbf{R}$. The direction vector is explicitly derived by

$$
\mathbf{a}(\theta)=\mathbf{R}_{\mathrm{n}} u_{\max }
$$

where $u_{\max }$ is the dominate eigenvector to the generalized eigenvalue equation $\mathbf{R} u_{\max }$ $=\lambda_{\max } \mathbf{R}_{\mathrm{n}} u_{\max }$

Elmer et al. (2012) gives the maximum signal to noise of a beamformer by maximizing the following expression

$$
\mathbf{w}_{\operatorname{maxSNR}}=\frac{\mathbf{w}^{\mathbf{H}} \mathbf{R}_{\mathrm{s}} \mathbf{w}}{\mathbf{w}^{\mathbf{H}} \mathbf{R}_{\mathrm{n}} \mathbf{w}}
$$

Here, the values contained within the weight vector $\mathbf{w}$ and its Hermition form are not yet known. The maximization of Equation 4.6 via taking the derivative with respect to $\mathbf{w}$ and setting the result equal to zero results is effectively finding the dominant eigenvector of the generalized eigenvalue equation

$$
\mathbf{R}_{\mathrm{s}} \mathbf{w}_{\operatorname{maxSNR}}=\lambda_{\max } \mathbf{R}_{\mathrm{n}} \mathbf{w}_{\operatorname{maxSNR}}
$$

A raw power value $P$ in units of counts at a particular $\nu$ and short term integration $(n)$ is measured by calculating

$$
P_{\nu, \mathrm{n}}=\mathbf{w}_{\operatorname{maxSNR}, \nu, \mathrm{n}}^{\mathrm{H}} \mathbf{R}_{\mathrm{s}, \nu, \mathrm{n}} \mathbf{w}_{\operatorname{maxSNR}, \nu, \mathrm{n}} .
$$


The max-SNR beamforming algorithm manipulates the individual dipole illumination patterns such that the aperture is uniformly illuminated for each formed beam. While this scheme produces the highest gain in a given direction, there is little control over the level of the sidelobes due to sharp transition in illumination pattern (recall that the theory of optics dictates that the far-field beams are related to the aperture illumination via a Fourier Transform). Nevertheless the high sensitivity over a large field of view is particularly advantageous for the detection of diffuse HI. A PAF that simultaneously form beams with reliably low sidelobe structure will decrease the survey times necessary to pursue the detection of cold gas accretion, the study of high velocity clouds (Moss et al., 2013) and the compact clouds being driven from the Galactic center (Di Teodoro et al., 2018).

\subsection{The FLAG Backend}

\subsubsection{General Overview}

The backend for the PAF was developed in collaboration with the Brigham Young University (BYU), West Virginia University (WVU) and the Green Bank Observatory (GBO). It consists of five high performance computing nodes (HPCs), each equipped with two Nvidia GeForce Titan X Graphical Processing Units (GPUs). Each HPC was connected to the Reconfigurable Open Architecture Computing Hardware $(\mathrm{ROACH})^{2}$ Field Programmable Gate Arrays (FPGA) boards and received one fifth of the total bandwidth of $151.59 \mathrm{MHz}$. Each HPC can run in three

\footnotetext{
${ }^{2}$ https://casper.berkeley.edu/wiki/ROACH-2_Revision_2
} 


\begin{tabular}{|c|c|c|c|c|c|c|c|c|c|}
\hline & 1 & 2 & 3 & 4 & $\ldots$ & 39 & 40 & $\ldots$ & 64 \\
\hline 1 & $R_{k}^{1,1}$ & $R_{k}^{1,2}$ & 0 & 0 & $\ldots$ & 0 & 0 & $\ldots$ & 0 \\
\hline 2 & $R_{k}^{2,1}$ & $R_{k}^{2,2}$ & 0 & 0 & $\ldots$ & 0 & 0 & $\ldots$ & 0 \\
\hline 3 & $R_{k}^{3,1}$ & $R_{k}^{3,2}$ & $R_{k}^{3,3}$ & $R_{k}^{3,4}$ & $\ldots$ & 0 & 0 & $\ldots$ & 0 \\
\hline 4 & $R_{k}^{4,1}$ & $R_{k}^{4,2}$ & $R_{k}^{4,3}$ & $R_{k}^{4,4}$ & $\ldots$ & 0 & 0 & $\ldots$ & 0 \\
\hline$\ldots$ & $\ldots$ & $\ldots$ & $\ldots$ & $\ldots$ & $\ldots$ & $\ldots$ & $\ldots$ & $\ldots$ & $\ldots$ \\
\hline 39 & $R_{k}^{39,1}$ & $R_{k}^{39,2}$ & $R_{k}^{39,3}$ & $R_{k}^{39,4}$ & $\ldots$ & $R_{k}^{39,39}$ & $R_{k}^{39,40}$ & $\ldots$ & 0 \\
\hline 40 & $R_{k}^{40,1}$ & $R_{k}^{40,2}$ & $R_{k}^{40,3}$ & $R_{k}^{40,4}$ & $\ldots$ & $R_{k}^{40,39}$ & $R_{k}^{40,40}$ & $\ldots$ & 0 \\
\hline 41 & 0 & 0 & 0 & 0 & $\ldots$ & 0 & 0 & $\ldots$ & 0 \\
\hline$\ldots$ & $\ldots$ & $\ldots$ & $\ldots$ & $\ldots$ & $\ldots$ & $\ldots$ & $\ldots$ & $\ldots$ & $\ldots$ \\
\hline 64 & 0 & 0 & 0 & 0 & $\ldots$ & 0 & 0 & $\ldots$ & 0 \\
\hline
\end{tabular}

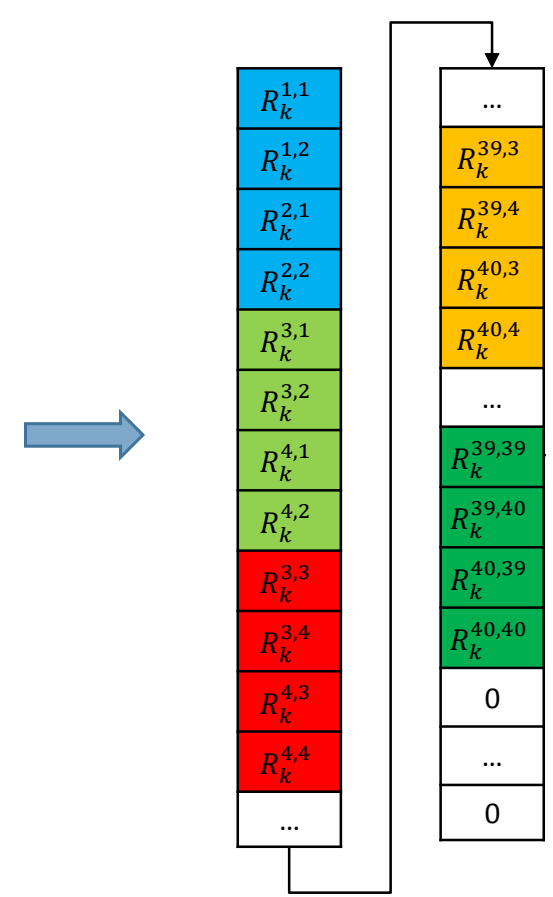

Figure 4.1 The structure of a covariance matrix used in beamforming. The numbers preceding each row/column correspond to the dipole element. Each element of the matrix stores the correlation between dipole elements $i$ and $j$ for a single frequency channel, $k$. The output is ordered in a flattened one-dimensional array that needs to be reshaped into a $40 \times 40$ matrix before beamforming weights can be applied. Additionally, due to xGPU limitations, the output size is $64 \times 64$, which results in many zeros that need to be thrown away in data processing. 
basic modes: (1) the calibration correlator mode (CALCORR) wherein the bandpass was made up of 500 discrete 'coarse' channels each $0.30318 \mathrm{MHz}$ wide; (2) The polyphase filter bank (PFB) correlator mode (PFBCORR) where a $30.318 \mathrm{MHz}$ (100 coarse channels) section of the original bandpass is selected to be sent through a PFB to obtain finer channelization. In this mode, a contiguous set of five coarse channels is output to 160 'fine' channels for a final frequency resolution of 9.47 $\mathrm{kHz}$; (3) the real-time beamformer mode (RTBF) where precomputed beamformer weights are read in and applied to save beamformed spectra to disk. This mode is designed to be used to detect transient sources such as pulsars and fast radio bursts. The remaining discussion will focus solely on the CALCORR and the PFBCORR modes.

In both correlator modes, each GPU runs two correlator threads making use of the xGPU library ${ }^{3}$, which is optimized to work on FLAG system parameters. Each correlator thread handles one-twentieth of the total bandwidth made up of either 25 non-contiguous coarse frequency channels or 160 contiguous fine channels and writes the raw output to disk in a FITS file format. The data acquisition software used to save these data to disk was borrowed from development code based for the Versatile GBT Astronomical Spectrometer (VEGAS) engineering FITS format. The output FITS file from each correlator thread is considered a 'bank' with a unique X-engine ID (XID; i.e., the correlator thread) ranging from 0 to 19 that is stored in the primary header of the FITS binary table.

The raw data output for both correlator modes are the covariance matrices

\footnotetext{
${ }^{3}$ https://github.com/GPU-correlators/xGPU/tree/master/src
} 
denoting the correlations of individual dipole elements. However, due to xGPU limitations, the covariance matrices are of size of $64 \times 64$ (with elements for row and column over 40 being set to zero) and flattened to a one-dimensional data vector whose length depends on the specific correlator mode. An example of how the

correlations are ordered is illustrated in Figure 4.1. Here, $\mathrm{R}_{\mathrm{k}}^{\mathrm{i}, \mathrm{j}}$ corresponds to the correlation between dipole $i$ and $j$ at frequency channel $k$. When in CALCORR mode, the bank file corresponding to XID ID 0 contains covariances matrices for frequency channels 0 to 4, 100 to $104, \ldots, 400$ to 404; the XID 1 bank file stores covariance matrices for frequency channels, 5 to 9,105 to $109, \ldots, 405$ to 409 . When in PFBCORR mode, the covariance matrices for channels 0 to 159 are stored in the bank file corresponding to XID 0 and continue in a linear and contiguous fashion such that the bank file corresponding to XID 19 stores data for frequency channels 3039 to 3199 . The logic during data reduction is to process each frequency channel individually then sort the result into the final bandpass based on the XID and mode in which the data were taken. The methods employed to construct the twodimensional form of the covariance matrices and sort the frequency channels are discussed in depth in Section 4.4.3.

\subsection{The Data and Observations}

Engineering and science commissioning observations were conducted over two separate observing runs in May and August of 2017 (GBT Project GBT16B_400). Calibration data obtained during the end of January 2017 as part of GBT projects 


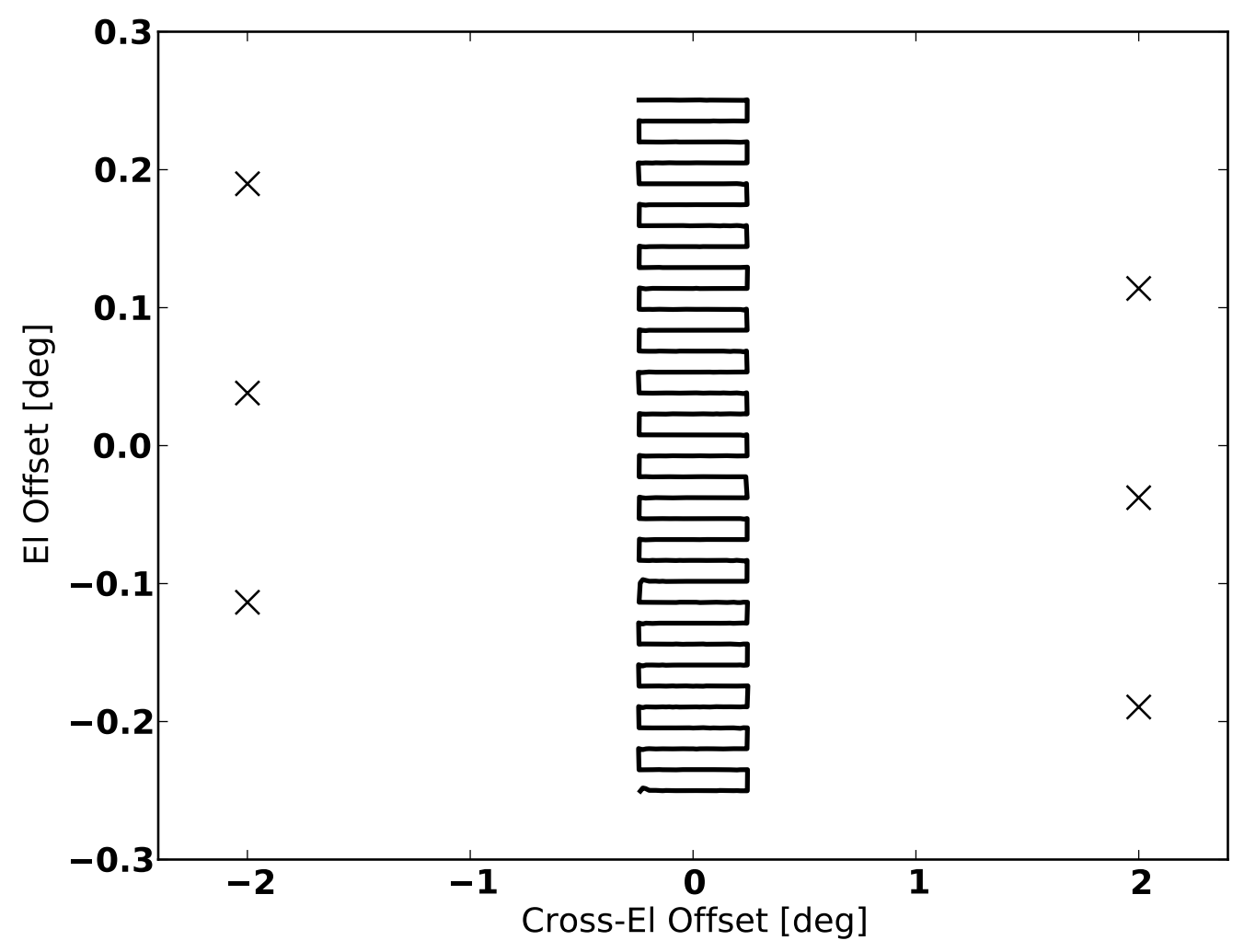

Figure 4.2 The trajectory of one of our calibration grids centered on 3C147. Offpointings are denoted by the $X$, while the solid black lines represent the trajectory of the grid.

GBT17B_360, GBT17B_455 (mapping of the Galactic center), and GBT18A_443 (mapping of a hydrogen recombination line region) are also included in the discussion regarding the quality of the beam former weights. The observations and backend setup covered in this work are summarized in Table 4.1.

\subsubsection{Calibration Observations}

To obtain measurements of $\mathbf{R}_{\mathrm{s}}$ and $\mathbf{R}_{\mathrm{n}}$ with which to derive maxSNR weights, we employ two primary calibration scans. A 'calibration grid' involves moving the GBT in a grid centered on a strong calibrator spanning 30 arcminutes in cross- 


\begin{tabular}{|c|c|c|c|c|c|c|c|c|c|}
\hline Session & UT Date & UT Start & UT End & Schedule Block Type & Source & Mode & Integration Length $[\mathrm{s}]$ & $\mathrm{LO}[\mathrm{MHz}]$ & Notes \\
\hline \multicolumn{10}{|l|}{ GBT16B_400_02 } \\
\hline & & & 1450.00000 & Continuous Trajectory \\
\hline GBT16B_400_03 & $2017-05-27$ & $04: 17: 55$ & 05:12:11 & Calibration Grid & 3С295 & CALCORR & 0.1 & 1450.00000 & Continuous Trajectory \\
\hline \multicolumn{10}{|l|}{ GBT16B_400_09 } \\
\hline & $2017-07-28$ & 05:06:19 & 05:38:52 & Calibration Grid & 3C295 & CALCORR & 0.5 & 1450.00000 & - \\
\hline \multicolumn{10}{|c|}{ GBT16B_400_12 } \\
\hline & $\begin{array}{l}2017-08-04 \\
2017-08-04\end{array}$ & $\begin{array}{l}04: 16: 54 \\
05: 30: 27\end{array}$ & $\begin{array}{l}05: 03: 33 \\
06: 02: 27\end{array}$ & $\begin{array}{l}\text { Calibration Grid } \\
\text { DecLatMap }\end{array}$ & $\begin{array}{c}\text { 3C295 } \\
\text { NGC6946 }\end{array}$ & $\begin{array}{l}\text { CALCORR } \\
\text { PFBCORR }\end{array}$ & $\begin{array}{l}0.5 \\
0.5\end{array}$ & $\begin{array}{l}1450.00000 \\
1450.00000\end{array}$ & $\begin{array}{c}40 \times 40 \square^{\prime} \\
41 \text { columns }\end{array}$ \\
\hline & $2017-08-04$ & $06: 12: 19$ & $06: 14: 53$ & 7Pt-Calibration & $3 \mathrm{C} 48$ & CALCORR & $\begin{array}{l}0.5 \\
0.5\end{array}$ & 1450.0000 & $10 \mathrm{~s}$ Tracks \\
\hline \multicolumn{10}{|l|}{ GBT16B_400_13 } \\
\hline & $2017-08-04$ & $13: 44: 40$ & 14:29:09 & Calibration Grid & $3 \mathrm{C} 123$ & CALCORR & 0.5 & 1449.84841 & - \\
\hline & 2017-08-04 & $14: 42: 46$ & $15: 14: 47$ & DecLatMap & NGC6946 & PFBCORR & 0.5 & 1449.84841 & 41 columns \\
\hline & $2017-08-04$ & $06: 12: 19$ & $06: 14: 53$ & 7Pt-Calibration & $3 \mathrm{C} 134$ & CALCORR & 0.5 & 1449.84841 & $15 \mathrm{~s}$ Tracks \\
\hline \multicolumn{10}{|c|}{ GBT16B_400_13 } \\
\hline & 2017-08-06 & $15: 54: 43$ & $15: 59: 43$ & OnOff & M31 Cloud 6 & PFBCORR & 0.5 & 1449.74271 & five $\min \mathrm{ON}$ scan \\
\hline & 2017-08-06 & 16:00:01 & $16: 05: 01$ & OnOff & M31 Cloud 6 & PFBCORR & 0.5 & 1449.74271 & five min OFF scan \\
\hline & 2017-08-06 & $16: 05: 29$ & $16: 20: 29$ & OnOff & M31 Cloud 6 & PFBCORR & 0.5 & 1449.74271 & five min ON scan \\
\hline & $2017-08-06$ & $16: 10: 37$ & $16: 15: 37$ & OnOff & M31 Cloud 6 & PFBCORR & 0.5 & 1449.74271 & five min OFF scan \\
\hline & 2017-08-06 & $16: 15: 55$ & $16: 20: 55$ & OnOff & M31 Cloud 6 & PFBCORR & 0.5 & 1449.74271 & five $\min O N$ scan \\
\hline & 2017-08-06 & $16: 21: 13$ & $16: 26: 13$ & OnOff & M31 Cloud 6 & PFBCORR & 0.5 & 1449.74271 & five min OFF scan \\
\hline & 2017-08-06 & $16: 26: 31$ & $16: 31: 31$ & OnOff & M31 Cloud 6 & PFBCORR & 0.5 & 1449.74271 & five $\min O N$ scan \\
\hline & $2017-08-06$ & 16:31:39 & $16: 36: 39$ & OnOff & M31 Cloud 6 & PFBCORR & 0.5 & 1449.74271 & five min OFF scan \\
\hline & 2017-08-06 & $16: 41: 15$ & $16: 43: 58$ & 7Pt-Calibration & 3C147 & CALCORR & 0.5 & 1450.0000 & $15 \mathrm{~s}$ Tracks \\
\hline & $2017-08-06$ & $16: 44: 48$ & $17: 22: 16$ & Calibration Grid & 3C147 & CALCORR & 0.5 & 1449.74271 & - \\
\hline \multicolumn{10}{|c|}{ GBT17B_360_01 } \\
\hline & 2018-01-27 & $15: 07: 59$ & $15: 09: 55$ & 7Pt-Calibration & $3 \mathrm{C} 295$ & CALCORR & 0.5 & 1450.0000 & $10 \mathrm{~s}$ Tracks \\
\hline & $2018-01-27$ & 15:11:00 & $15: 39: 18$ & Calibration Grid & 3С295 & CALCORR & 0.5 & 1450.0000 & - \\
\hline & $2018-01-27$ & $15: 40: 29$ & $15: 42: 24$ & 7Pt-Calibration & 3C295 & CALCORR & 0.5 & 1450.00000 & $10 \mathrm{~s}$ Tracks \\
\hline \multicolumn{10}{|c|}{ GBT17B_360_02 } \\
\hline & 2018-01-27 & $18: 32: 59$ & $18: 36: 07$ & 7Pt-Calibration & $3 \mathrm{C} 295$ & CALCORR & 0.5 & 1450.00000 & 10 s Tracks; \\
\hline & $2018-01-27$ & 19:13:57 & $19: 41: 40$ & Calibration Grid & 3C147 & CALCORR & 0.5 & 1450.00000 & - \\
\hline & $2018-01-27$ & 21:07:00 & 21:10:04 & 7Pt-Calibration & $3 \mathrm{C} 147$ & CALCORR & 0.5 & 1450.00000 & $10 \mathrm{~s}$ Tracks \\
\hline \multicolumn{10}{|l|}{ GBT17B_360_03 } \\
\hline & $2018-01-28$ & $06: 44: 29$ & $06: 47: 38$ & 7Pt-Calibration & $3 \mathrm{C} 295$ & CALCORR & 0.5 & 1449.84841 & $10 \mathrm{~s}$ Tracks \\
\hline & $2018-01-28$ & $06: 48: 56$ & $07: 17: 23$ & Calibration Grid & 3C295 & CALCORR & 0.5 & 1449.84841 & - \\
\hline & $2018-01-28$ & 08:05:49 & $08: 36: 44$ & DecLatMap & NGC4258 Field & PFBCORR & 0.5 & 1449.84841 & 31 columns \\
\hline & $2018-01-28$ & 08:05:49 & $08: 36: 44$ & DecLatMap & NGC4258 Field & PFBCORR & 0.5 & 1449.84841 & 31 columns \\
\hline & $2018-01-28$ & $08: 38: 28$ & 09:07:35 & DecLatMap & NGC4258 Field & PFBCORR & 0.5 & 1449.84841 & 31 columns \\
\hline \multicolumn{10}{|l|}{ GBT17B_360_04 } \\
\hline & 2018-01-29 & $07: 29: 58$ & $08: 32: 14$ & Calibration Grid & 3C295 & CALCORR & 0.5 & 1450.00000 & - \\
\hline & 2018-01-29 & $08: 38: 51$ & 08:42:10 & 7Pt-Calibration & 3C295 & CALCORR & 0.5 & 1450.00000 & 20 s Tracks \\
\hline & 2018-01-29 & $08: 50: 26$ & $09: 20: 42$ & DecLatMap & NGC4258 Field & PFBCORR & 0.5 & 1450.0000 & 31 columns \\
\hline & 2018-01-29 & 09:25:19 & $09: 56: 10$ & DecLatMap & NGC4258 Field & PFBCORR & 0.5 & 1450.0000 & 31 columns \\
\hline & 2018-01-29 & 09:59:00 & $10: 28: 50$ & DecLatMap & NGC4258 Field & PFBCORR & 0.5 & 1450.00000 & 31 columns \\
\hline & 2018-01-29 & $10: 30: 44$ & $10: 59: 11$ & DecLatMap & NGC4258 Field & PFBCORR & 0.5 & 1450.00000 & 31 columns \\
\hline \multicolumn{10}{|c|}{ GBT17B_360_05 } \\
\hline & 2018-01-30 & $12: 02: 53$ & 12:13:08 & 7Pt-Calibration & 3C 295 & CALCORR & 0.5 & 1450.0000 & $20 \mathrm{~s}$ Tracks \\
\hline & 2018-01-30 & $12: 18: 09$ & $12: 50: 55$ & DecLatMap & NGC4258 Field & PFBCORR & 0.5 & 1450.00000 & 31 columns \\
\hline & 2018-01-30 & $12: 53: 24$ & $13: 00: 44$ & 7Pt-Calibration & 3С 295 & CALCORR & 0.5 & 1450.00000 & $20 \mathrm{~s}$ Tracks \\
\hline GBT17B_360_06 & & & & & & & & & \\
\hline & 2018-02-03 & 17:30:03 & $17: 35: 46$ & 7Pt-Calibration & $3 \mathrm{C} 48$ & CALCORR & 0.5 & 1075.00000 & $30 \mathrm{~s}$ Tracks \\
\hline & 2018-02-03 & $18: 15: 50$ & $18: 21: 39$ & 7Pt-Calibration & $3 \mathrm{C} 48$ & CALCORR & 0.5 & 1250.00000 & $30 \mathrm{~s}$ Tracks \\
\hline & 2018-02-03 & $18: 32: 32$ & $18: 38: 21$ & 7Pt-Calibration & $3 \mathrm{C} 48$ & CALCORR & 0.5 & 1350.00000 & 30 s Tracks \\
\hline & 2018-02-03 & 18:51:01 & $18: 56: 52$ & 7Pt-Calibration & $3 \mathrm{C} 48$ & CALCORR & 0.5 & 1550.00000 & 30 s Tracks \\
\hline & 2018-02-03 & $19: 08: 18$ & 19:14:11 & 7Pt-Calibration & $3 \mathrm{C} 48$ & CALCORR & 0.5 & 1650.00000 & $30 \mathrm{~s}$ Tracks \\
\hline & 2018-02-03 & $19: 25: 22$ & $19: 31: 17$ & 7Pt-Calibration & $3 \mathrm{C} 48$ & CALCORR & 0.5 & 1750.00000 & $30 \mathrm{~s}$ Tracks \\
\hline & 2018-02-03 & $19: 57: 26$ & $20: 03: 28$ & 7Pt-Calibration & $3 \mathrm{C} 48$ & CALCORR & 0.5 & 1449.74271 & 30 s Tracks \\
\hline & $2018-02-03$ & $20: 04: 47$ & $20: 35: 45$ & Calibration Grid & $3 \mathrm{C} 48$ & CALCORR & 0.5 & 1449.74271 & - \\
\hline & 2018-02-03 & $20: 46: 10$ & $20: 53: 30$ & OnOff & M31 Cloud 6 & PFBCORR & 0.5 & 1449.74271 & ON scan \\
\hline & 2018-02-03 & $20: 53: 30$ & - & OnOff & M31 Cloud 6 & PFBCORR & 0.5 & 1449.74271 & OFF scan \\
\hline GBT17B_360_07 & & & & & & & & & \\
\hline & 2018-02-05 & $06: 25: 20$ & $06: 53: 49$ & Calibration Grid & 3C 295 & CALCORR & 0.5 & 1450.00000 & - \\
\hline & 2018-02-05 & 07:36:30 & 08:07:31 & DecLatMap & NGC4258 Field & PFBCORR & 0.5 & 1450.00000 & 31 columns \\
\hline & 2018-02-05 & 08:09:00 & 08:37:47 & DecLatMap & NGC4258 Field & PFBCORR & 0.5 & 1450.00000 & 31 columns \\
\hline & 2018-02-05 & $08: 39: 38$ & 09:09:15 & DecLatMap & NGC4258 Field & PFBCORR & 0.5 & 1450.00000 & 31 columns \\
\hline & 2018-02-05 & 09:44:19 & $10: 00: 11$ & DecLatMap & NGC4258 Field & PFBCORR & 0.5 & 1450.0000 & columns 7 to 23 \\
\hline & $2018-02-05$ & 10:04:05 & $10: 14: 38$ & 7Pt-Calibration & $3 \mathrm{C} 295$ & CALCORR & 0.5 & 1450.00000 & $60 \mathrm{~s}$ Tracks \\
\hline
\end{tabular}

Table 4.1 Summary of FLAG Observations

\begin{tabular}{lcccc}
\hline \hline Beam & GBT16B_400 Offset XEL ['] & GBT16B_400 Offset EL ['] & GBT17B_360 XEL Offset ['] & GBT17B_360 EL Offset ['] \\
\hline 0 (Boresight) & 0 & 0 & 0 & 0 \\
1 & -4.55 & 7.88 & -2.28 & 3.94 \\
2 & 4.55 & 7.88 & 2.28 & 3.94 \\
3 & 4.55 & 0 & 2.28 & 0 \\
4 & 4.55 & -7.88 & 2.28 & -3.94 \\
5 & -4.55 & -7.88 & -2.28 & -3.94 \\
6 & -4.55 & 0 & -2.28 & 0 \\
\hline
\end{tabular}

Table 4.2 Formed Beam Offsets 
elevation (XEL) as set by the horizontal celestial coordinate system (i.e. 'Encoder' setting when using the GBT) and 34 rows spaced 0.91 arcminutes (approximately 1/10 the FWHM of the GBT beam at $1.4 \mathrm{GHz}$ ) apart in Elevation (EL). We compute $\mathbf{R}_{\mathrm{n}}$ by tracking two degrees in XEL away from the grid for a duration of ten seconds. We track after every fifth row to attain six total reference pointings with three evenly spaced on each side of the grid. To ensure adequate spatial sampling, we move the telescope at a rate of 0.91 arcminutes per second and dump integrations to disk every one second. The trajectory of the calibration grid observations performed during session 14 of GBT16B_400 centered on 3C147 is shown in Figure 4.2 as the solid black lines, and the $\mathrm{x}$ symbols representing the mean location of the reference pointings. Each integration within the grid can be paired with the closest reference pointing to derive an independent beam. The result is many independent beams over the entire 30 arcminute field of view (FOV) of the instrument that we can use to create a customized beam pattern. For both projects, we utilized a beam pattern that consisted of seven total beams arranged as a single boresight beam with the outer six beams placed as a symmetric hexagon. The offsets of each beam for each project is listed in Table 4.4.1. The compact offsets of GBT17B_360 relative to those for GBT16B_400 allows for the beam pattern to be exactly Nyquist sampled assuming a 9.1' FWHM for a single beam.

While it is interesting to obtain detailed spatial information of the array response provided by a calibration grid, the necessary $\sim 30$ minutes of total observing time (including overhead) is disadvantageous. In the instances where beam offsets are explicitly chosen and seven total beams are desired, a seven point calibration 
scan (or 7Pt-Cal), can be performed. This procedure involves (1) tracking the area of sky minus two degrees in XEL away from the source and at the same EL offset as the centers for beams five and six; (2) directly tracking the source (i.e. the boresight or zeroth beam); (3) slew the telescope such that the calibrator source is located at the desired center of beams one through six; (4) tracking the area of sky minus two degrees away from the source and at the same EL offsets as the centers of beams one and two. The two reference pointings at similar EL offsets as the outer beams allow for construction of $\mathbf{R}_{n}$ (while also accounting for elevation-dependent effects), while the tracks on the desired beam centers collects the necessary response data to derive maxSNR weights. The duration of each track ranges between 10 and 30 seconds.

\subsubsection{Science Observations}

The science data were collected using two distinct observing modes: (1) steering the telescope along columns of declination $\left(\delta_{\mathrm{J} 2000}\right)$ positioned at constant right ascension $\left(\alpha_{\mathrm{J} 2000}\right)$ coordinates to make on-the-fly maps and $(2)$ position switching wherein the telescope first tracks the source, then tracks a reference position two degrees away in $\alpha_{\mathrm{J} 2000}$. These observing strategies were respectively executed using the available observing procedures of DecLatMap and OnOff.

The columns of $\delta_{\text {J2000 }}$ making up a DecLatMap are spaced every $3^{\prime}$ while dumping data every $1.67^{\prime}$ to ensure adequate spatial Nyquist sampling. For consistency with the calibration grid, the maps of NGC6946 were observed in Encoder (horizon- 
tal coordinates) and extended two degrees in XEL and EL. There were a total of 41 scans in elevation. The maps of NGC6946 are centered on $\alpha_{\mathrm{J} 2000}=20^{h} 34^{m} 52.3^{s}$, $\delta_{\mathrm{J} 2000}=+60^{\circ} 09^{\prime} 14^{\prime \prime}$.

The OnOff observations were all performed on the faint Hi source, M31 Cloud 6, which was first discovered by Wolfe et al. (2013). During the fourteenth session of project GBT16B_400, each On-Off pair consisted of a five minute ON and five minute OFF scan to ensure equivalent noise properties. Note that the local oscillator is set to a specific topocentric frequency of $1449.74271 \mathrm{MHz}$ in an attempt to center the source in a coarse channel. All spectral line observations were performed in the backend mode of PFBCORR to obtain a velocity resolution of $9.47 \mathrm{kHz}(\sim 1.9 \mathrm{~km}$ $\mathrm{s}^{-1}$ in radial velocity).

\subsubsection{Data Reduction}

A given scan done with FLAG produces several types of ancillary FITS files written to /home/gbtdata/PROJECT_SESSION that contain important metadata such as the antenna positions. These metadata must be collated and combined with the raw correlations stored in the 20 bank FITS files to create a single dish FITS (SDFITS) file. This process is carried out by the sdfits program available on the GBO computing network. The unique format of the raw FLAG data necessitate custom software that not only collates all the metadata similar to sdfits, but also performs the post-correlation beamforming. This section summarizes the data reduction processes and pipeline for FLAG spectral line data. 


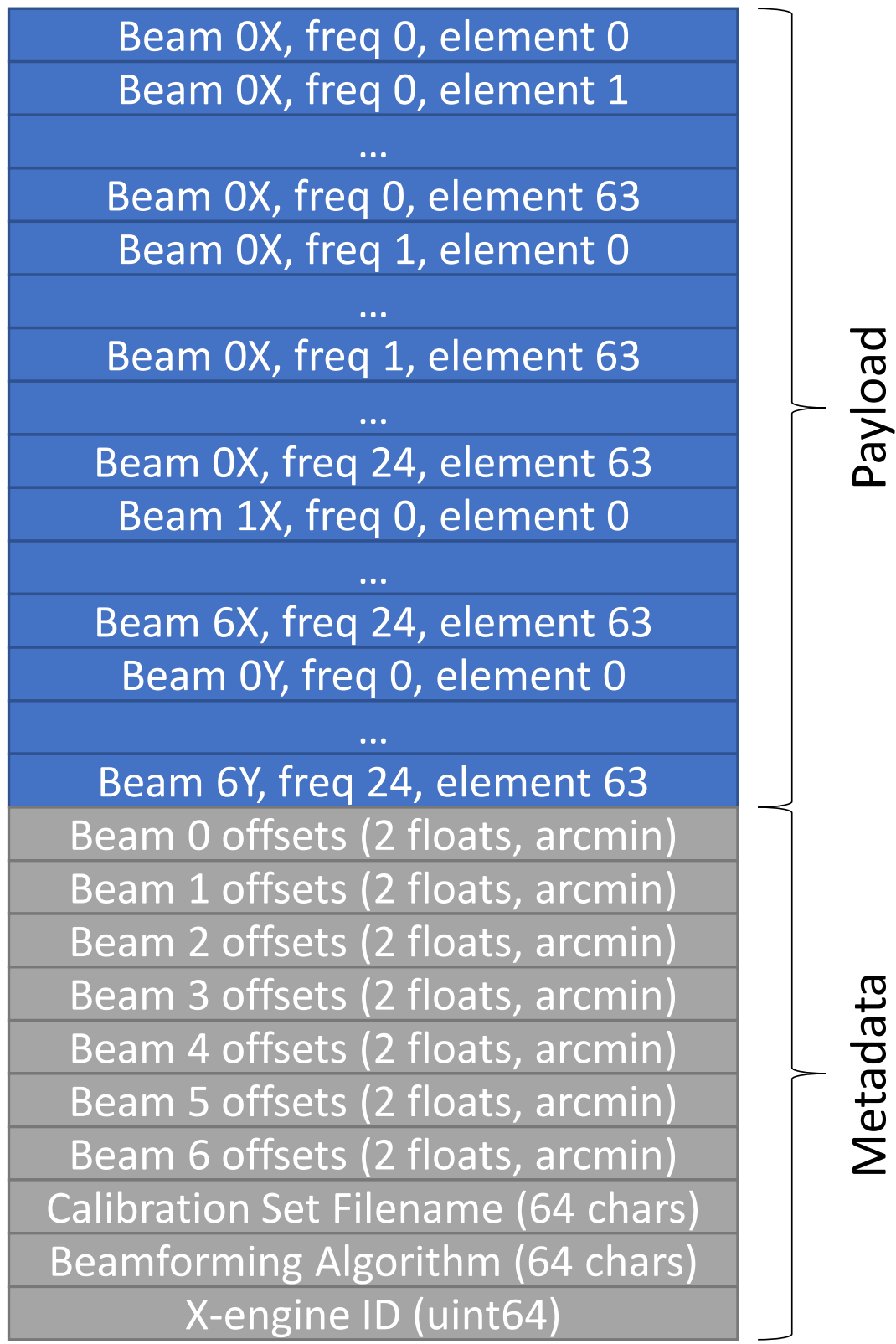

Figure 4.3 The structure of the binary files that contain the complex beamforming weights (Black 2018; private communication). 


\subsubsection{Processing Beamforming Weights}

The first step in the filling process is to convert a set of 20 binary files (one for each bank) that stores the complex beamformer weights to FITS files to be accessed later to create the beamformed bandpasses. This is done with the script, Weight_Filler.py, that is a part of the utils available through the custom SpectralFiller Python package ${ }^{4}$. The primary purpose of this software suite is to generate a FITS file containing raw beamformed spectra in the units of counts. Figure 4.3 summarizes the format of these binary files. The first component of the file contains (in sequential order) the real and imaginary component of the complex weight for each polarization, beam, coarse frequency channel, and dipole element. Recall that the correlation matrices are of shape $64 \times 64$. Since we are only interested in the first 40 elements that correspond to the 40 available data ports $(19 \times 2$ dipoles (dual polarizations) with two spare ports), the remaining elements are discarded. Once the FITS files that contain the weights are generated The 'main' script that creates an SDFITS file is PAF_Filler.py. This script processes each bank FITS file individually by reading in the associated weights and bank FITS files (identified through a common XID), computes Equation 4.8 for each frequency channel, and sorts the result into contiguous bandpass (recall that the data stored in the raw bank FITS files do not correspond to adjacent frequency channels). Aside from the post correlation beamforming, this script also collates all the necessary metadata and applies the beam offsets.

As of Spring 2018, the GBO LO and IF system managers cannot explicitly

\footnotetext{
${ }^{4}$ https://github.com/nipingel/SpectralFiller
} 
communicate with the new beamforming backend. In other words, this means the frequencies recorded for any observation are only recorded in the topocentric reference frame. However, since the RA/Dec values of each beam pointing center are available after the beam offsets are applied, a proper Doppler correction can be calculated. Currently the Doppler correction will be applied such that the IF frequencies will be in the Heliocentric reference frame with the OPTICAL velocity definition. Translations to other reference frames and velocity definitions (e.g., LSRK in the RADIO definition) can be performed in GBTIDL during further calibration. The code that performs the correction is an edited version of Frank Ghigo's radial velocity correction calculator ${ }^{5}$. The final SDFITS file contains the raw beamformed spectra in units of 'counts' that can be manipulated within the GBTIDL environment exactly in the same manner as data from the VEGAS backend.

\subsubsection{Calibration and Imaging}

Calibration begins by calculating the equivalent system flux density (SEFD) from the seven-point and grid scans. This quantity is the flux density equivalent of $T_{\text {sys }}$. The SEFD is convenient because it directly folds in the directly measurable $T_{\text {sys }} / \eta$ ratio. Knowing the flux density of a source observed with a grid schedule block, $S_{\text {CalSrc }}$ the system equivalent flux density is given by

$$
S_{\mathrm{SEFD}}=\frac{S_{\mathrm{CalSrc}}}{\left(\frac{P_{\mathrm{On}}}{P_{\mathrm{Off}}}-1\right)},
$$

\footnotetext{
${ }^{5}$ http://www.gb.nrao.edu/GBT/setups/radvelcalc.html
} 
where $P_{\text {On }}$ and $P_{\text {Off }}$ are respectively the On and Off power values. Two distributions of On and Off raw beamformed power values are built from the values generally contained between coarse channels corresponding to $1400.2 \mathrm{MHz}$ to $1416.6 \mathrm{MHz}$ and 1425.1 MHz to $1440.3 \mathrm{MHz}$ to avoid Galactic Hi emission and fit with separate Gaussian functions. $P_{\mathrm{On}}$ is taken to be the maximum value in the On distribution, while $P_{\text {Off }}$ is taken as the fitted mean of the Off distribution. The respective uncertainties are the fitted rms given by the fit to the distinct distributions. All power values are corrected for atmospheric attenuation. The final uncertainty for the SEFD value is computed by propagating the statistical uncertainties of $P_{\text {On }}$, $P_{\text {Off }}$, and $S_{\text {CalSrc }}$. The flux density of a given calibrator source is taken from Perley \& Butler (2017). The flux density of a target source is then given by

$$
S_{\mathrm{src}}=S_{\mathrm{SEFD}}\left(\frac{P_{\mathrm{On}}}{P_{\mathrm{Off}}}-1\right)
$$

Similar to the calibration procedure outlined in Section 2.3.1, we obtain a reference spectrum to use as $P_{\text {Off }}$ from the edges of our science maps by taking the mean power in each frequency channel for the first and last four integrations of a particular DecLatMap scan. $P_{\mathrm{On}}$ in Equation 1.10 is then the raw power in each integration recording during the DecLatMap procedure. The system equivalent flux densities used to scale the raw power ratios for each beam and each session are summarized in Table 1.4. The mean system equivalent flux density over all beams and the presented data is $14.40 \pm 0.65 \mathrm{Jy} /$ beam. For the sake of direct comparison if we assume a gain of $1.86 \mathrm{~K} / \mathrm{Jy}$ as derived in Section 2.3.1, this translates to a mean $T_{\text {sys }}$ of about $27 \mathrm{~K}$. 
While this assumption does not consider specific parameters of the FLAG receiver, such as the the large spillover from the illumination pattern of individual dipoles and their mutual coupling, it is encouraging to see a very similar $T_{\text {sys }}$ to that of the existing single pixel receiver $(\sim 18 \mathrm{~K})$. The $T_{\text {sys }}$ of FLAG and its overall sensitivity will be discussed further in Section 1.5.1.

An example of a raw and calibrated integration when the system is in PFBCORR mode is shown in Figure 1.4. The nulls, or 'scalloping', seen every 303.18 $\mathrm{kHz}$ (every 32 fine frequency channels) in the top plot is an artifact caused by the two stage PFB architecture approach currently implemented in the backend. As the raw complex time series data are processed within the ROACHs, a response filter is implemented in the coarse PFB such that the adjacent channels overlap at the $3 \mathrm{~dB}$ point to reduce spectral leakage. This underlying structure becomes readily apparent after the fine PFB implemented in the GPUs, however. The scalloping therefore traces the structure of each coarse channel across the bandpass. While the structure is somewhat mitigated in the calibrated data (since there is a division by a reference spectrum), power variations caused by spectral leakage (power from adjacent channels) in the transition bands of the coarse channel bandpass filter result in residual structure. Additionally, this scheme leads to signal aliasing stemming from the overlap in coarse channels. This does not hinder the performance of FLAG in terms of sensitivity, but a fix for the signal aliasing is a top priority going forward. One suggested future scheme is to double the input coarse channel into the PFB such that the drop in power caused by the $3 \mathrm{~dB}$ overlap is avoided.

The scalloping can be completely mitigated by dithering the frequency such 


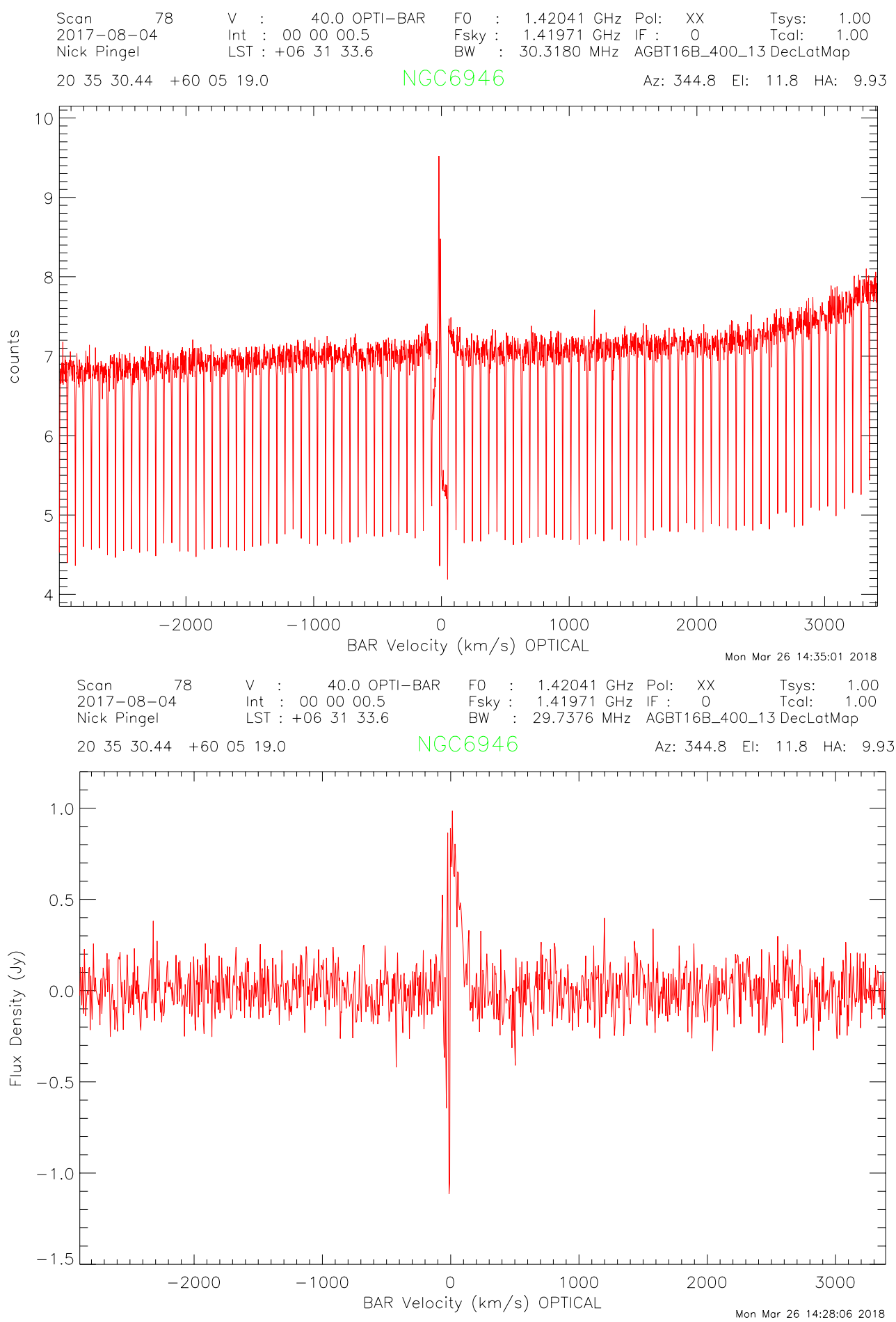

Figure 1.4 Top: An example of an uncalibrated, beamformed spectrum as seen in a GBTIDL plotter window taken from the 35th integration of the 19th column of a DecLatMap scan of NGC6946. The $3 \mathrm{~dB}$ drop in power (i.e., 'scalloping') is an artifact of the two step PFB implementation of the backend (see text). Bottom: The calibrated version of the above spectrum. While the scalloping behavior appears to be mitigated, the signal aliasing at the edge of a coarse channel is still present. 
that a subsequent map has a central frequency that is either $151.59 \mathrm{kHz}$ (or onehalf of a coarse channel width) above or below the initial LO setting. In the case of NGC6946, the LO was shifted by $-151.59 \mathrm{kHz}$ to be at $1449.84841 \mathrm{MHz}$. Before the integrations from a science observation are correctly scaled to units of Jy, the six fine frequency channels that span adjacent coarse channel edges and are thus affected by the scalloping are blanked. The exact shift necessary to fill in the blanked regions is computed and applied to the spectra with the higher LO setting. The final flux value in a given frequency channel is the weighted average of the original fluxes from the individual low and high frequency dithered mapping scans, where the weights are nominally the inverse squared equivalent system flux densities. It is somewhat common for a correlator thread to terminate (or drop out) due to issues with the data throughput, which ultimately leads to blank discontinuities in the spectra that span the width of a coarse channel, or $303.18 \mathrm{kHz}$ and blank flux values. If a flux value from any of the contributing frequency dithered spectra are blank due to a drop out, a weight of 0 is adopted. The final weighted spectra are then smoothed to a velocity resolution of $5.2 \mathrm{~km} \mathrm{~s}^{-1}(24.414 \mathrm{kHz})$. The data from separate beams are combined using the AIPS task DBCON and imaged using a convolution function of a Gaussian-tapered circular Bessel function available in the task SDGRD. Note that due to problems with dead dipole elements, only weights for the XX polarization are used in the beamforming of the science observations presented in this chapter, but the behavior of the YY polarization is still analyzed for the calibration data. 


\subsection{Results}

The important aspects of the spectral line modes of FLAG to explore with the science observations outlined above are:

- How the $T_{\text {sys }}$ (or $S_{\mathrm{SEFD}}$ ) of individual beams behave over time?

- How well these values compare with the single pixel receiver

- How does the noise behave with what is expected theoretically?

- In turn, what is the overall increase in the survey speeds?

- How do the FLAG maps compare to maps made with the single pixel receiver in terms of spatial coincidence of emission, total flux, and detection of low column density features?

\subsection{1 $T_{\text {sys }}$ and Noise Properties}

By observing some reference position in addition to an 'On source' scan, the theoretical signal-to-noise ratio $(\mathrm{S} / \mathrm{N})$ is given by

$$
\frac{T_{\mathrm{a}}}{\sigma}
$$

where $T_{\mathrm{a}}$ is the antenna temperature for the On source observation and $\sigma$ is the theoretical rms noise determined by the ideal radiometer equation

$$
\sigma=\frac{T_{\mathrm{sys}}}{\sqrt{N_{\mathrm{pol}} \Delta \nu t_{\mathrm{eff}}}},
$$


where $N_{\text {pol }}$ is the number of polarizations, $\Delta \nu$ represents the frequency width of an individual channel, and $t_{\text {eff }}$ is the effective integration time (Condon \& Ransom, 2016). $T_{a}$ in the above equation is related to the intensity of incident radiation through the blackbody equation in the Rayleigh-Jeans limit

$$
I_{\mathrm{src}}=\frac{2 k T_{\mathrm{a}}}{\lambda}
$$

The total power $P_{\text {src }}$ incident (from an unpolarized source) at some wavelength $\lambda$ on an antenna is the $I_{s r c}$ detected over some infinitesimal, physical area $\mathrm{d} A$ measured over the solid angle of the source, $d \Omega$, in steradians

$$
P_{\mathrm{src}}=I_{\mathrm{src}} d A d \Omega=\left(\frac{2 k T_{\mathrm{a}}}{\lambda^{2}}\right)(d A d \Omega)
$$

Knowing that that $(d A d \Omega)=\lambda^{2}$ from the Antenna Theorem (Condon \& Ransom, 2016), the expression for the source power received by the telescope is then

$$
P_{\mathrm{src}}=2 k T_{\mathrm{a}} \text {. }
$$

Substituting this result into the left-hand side and integrating up to the average effective area $A_{e}$ that measures the total area of the primary reflector illuminated by the feed and beamsize $\Omega_{B}$ reduces Equation 1.14 to

$$
2 k T_{\mathrm{a}}=I_{\mathrm{src}} A_{\mathrm{e}} \Omega_{\mathrm{B}}
$$


Finally, knowing that the flux is $I_{s r c}$ over the beam $\Omega_{\mathrm{B}}$, and putting $A_{\mathrm{e}}$ in terms of the aperture efficiency, $\eta$, which is essentially a measure of how well a particular feed illuminates the collecting surface, the geometric area $A_{g}$, an expression for the $T_{\mathrm{a}}$ can be obtained such that

$$
T_{\mathrm{a}}=\frac{S_{\mathrm{src}} A_{\mathrm{g}} \eta}{2 k}
$$

If ideal observations of blank sky are being recorded, the measured $T_{\mathrm{a}}$ is approximately equal to $T_{\text {sys }}$ and Equation 1.17 becomes

$$
\frac{T_{\mathrm{sys}}}{\eta}=\frac{S_{\mathrm{SEFD}} A_{\mathrm{g}}}{2 k}
$$

Substituting the definition for $S_{\mathrm{SEFD}}$ in from Equation 4.9 and putting the power levels in terms of the product between correlation matrices and beamforming weights from Equation 4.8 results in the expression

$$
\frac{T_{\text {sys }}}{\eta}=\frac{S_{\text {CalSrc }} A_{\mathrm{g}}}{2 k} \frac{\mathbf{w}^{\mathrm{H}} \mathbf{R}_{\mathrm{n}} \mathbf{w}}{\mathbf{w}^{\mathrm{H}} \mathbf{R}_{\mathrm{s}} \mathbf{w}}
$$

This equation is an oft-used metric to compare the performance of different PAFs (Jeffs et al., 2008; Landon et al., 2010; Roshi et al., 2018) as it can be directly measured. Since each electronically formed beam has a unique $S_{\mathrm{SEFD}}$, a $T_{\mathrm{sys}} / \eta$ value can be also be measured for each beam.

Figure 1.5 shows how $T_{\text {sys }} / \eta$ typically varies across the bandpass for a single beam (in this case the boresight beam). A characteristic value is computed by taking the mean between frequencies of 1400.2 to $1416.6 \mathrm{MHz}$ and 1425.1 to 1440.3 


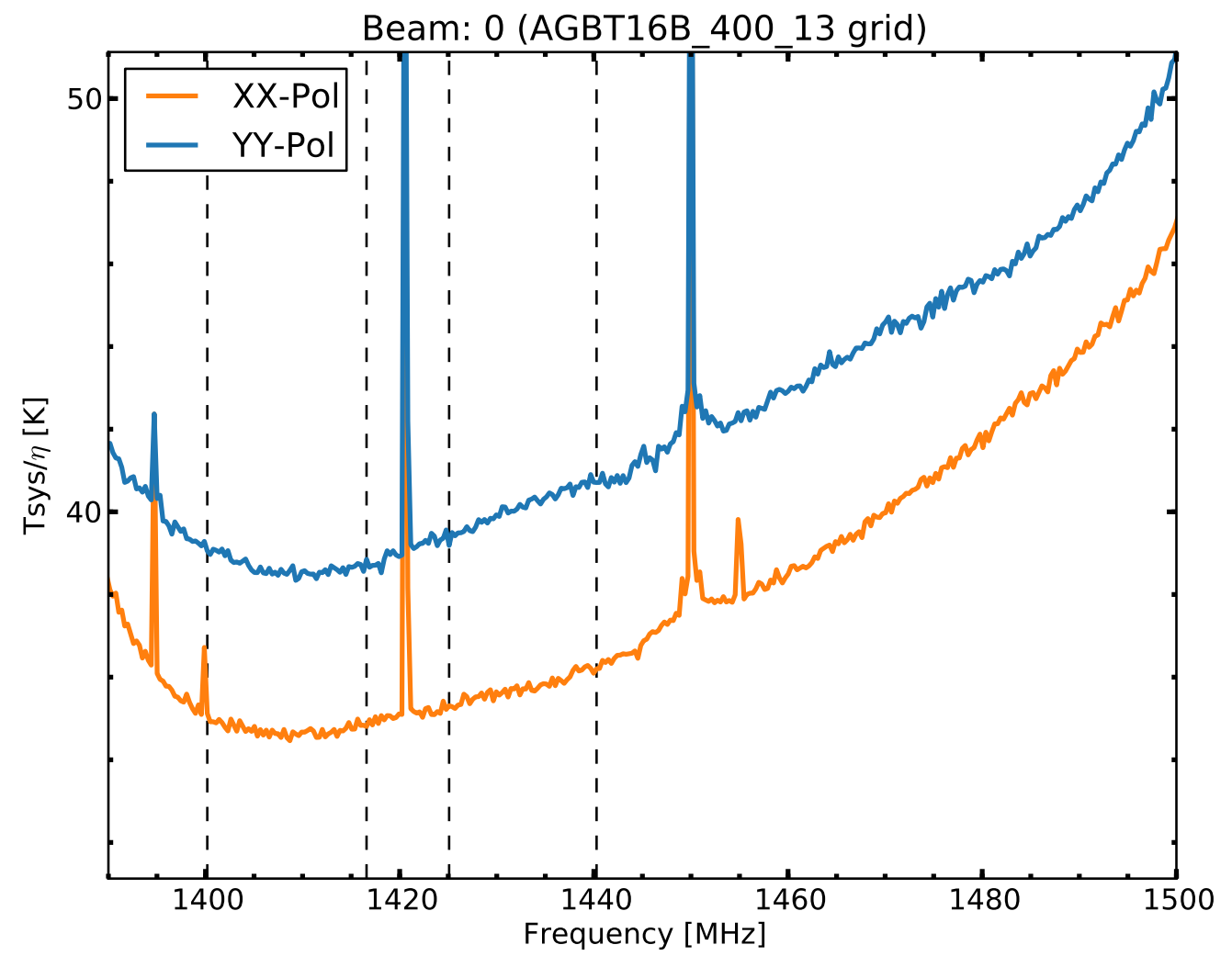

Figure $1.5 T_{\text {sys }} / \eta$ for the boresight beam from a grid calibration observation of 3C295 from 16B_400_13. The vertical dashed lines enclose the frequencies over which the mean value was computed. Note that the Galactic Hi frequencies are excluded from this calculation. The spike at $1450 \mathrm{MHz}$ is due to the DC component of the Fourier Transform of the complex time series data. 


\begin{tabular}{lccc}
\hline \hline Beam & Mean $T_{\text {sys }} / \eta(\mathrm{XX})[\mathrm{K}]$ & Mean $T_{\text {sys }} / \eta(\mathrm{YY})[\mathrm{K}]$ & Mean $S_{\text {sys }}[$ Jy $/$ beam $](\mathrm{XX})$ \\
\hline 0 (Boresight) & $35.70 \pm 0.49$ & $44.91 \pm 0.97$ & $12 \pm 1$ \\
1 & $34.70 \pm 0.49$ & $39.51 \pm 0.97$ & $13 \pm 1$ \\
2 & $34.90 \pm 0.58$ & $46 \pm 2$ & $12 \pm 1$ \\
3 & $35.04 \pm 0.80$ & $53 \pm 1$ & $14 \pm 2$ \\
4 & $39.77 \pm 0.71$ & $52 \pm 2$ & $14 \pm 2$ \\
5 & $36.02 \pm 0.60$ & $44.31 \pm 0.76$ & $14 \pm 2$ \\
6 & $37.72 \pm 0.71$ & $41.44 \pm 0.64$ & $14 \pm 2$ \\
\hline
\end{tabular}

Table 1.3 Mean $T_{\text {sys }} / \eta$ over all sessions

MHz. Again, two distinct frequencies ranges are used to avoid Galactic Hi emission near the $1420.4 \mathrm{MHz}$. In principle, each raw count value used to beamformed power ratio in Equation 1.19 value for a discrete frequency channel bin will have an associated error similar to the SEFD; however, as these were not readily available the associated uncertainties for the quoted $T_{\mathrm{sys}} / \eta$ in this dissertation are purely the statistical uncertainties from computing the characteristic value. The increase of $T_{\text {sys }} / \eta$ towards the edge of the bandpass is due to the roll-off in the analog filter where gain will decrease (in turn causing an increase in $T_{\text {sys }}$, while the large spike at $1450 \mathrm{MHz}$ is due to the DC component of the Fourier Transform of the raw complex time series data.

Two major aspects regarding the overall performance of FLAG are how $T_{\text {sys }} / \eta$ for each distinct beam changes as function of time and the length of time the complex beamforming weights stay valid. Figure 1.6 shows the temporal variation of $T_{\text {sys }} / \eta$ for the boresight beam. The points are taken from all available calibration data over all sessions. The abscissa values of duration are computed by taking the mean decimal modified Julian date (DMJD) during a given calibration scan. The reference DMJD is taken to be the first calibration scan of the respective observing run. 


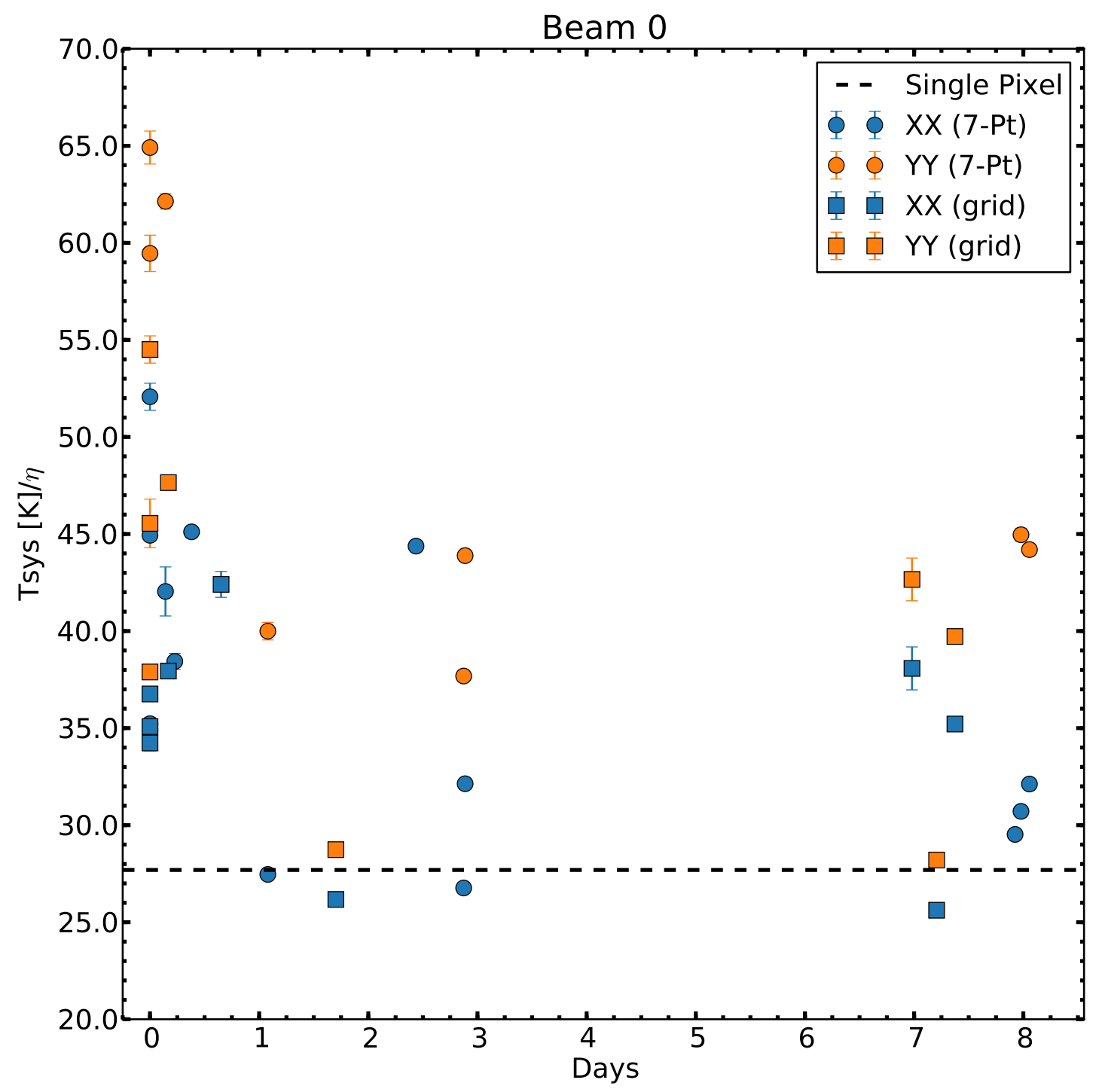

Figure 1.6 Temporal variation of $T_{\text {sys }} / \eta$ for the boresight beam. The circle points denote values derived from a seven-point calibration, while the square points are computed from grid calibrations. The general trend of high values for the YY polarization, while the XX polarization is relatively stable over the duration of a given observing run, is true for all outer beams. The calibration data shown here is taken from all observing runs. The horizontal dashed line represents the value estimated for the existing single-pixel feed. 


\begin{tabular}{|c|c|c|c|c|c|c|}
\hline Session & Beam & Scan Type & $T_{\mathrm{sys}} / \eta[\mathrm{K}]$ & $\mathrm{S}_{\text {sys }}[\mathrm{Jy} / \mathrm{beam}]$ & Calibration Source & Source Flux [Jy] \\
\hline \multicolumn{7}{|c|}{ GBT16B_400_12 } \\
\hline & 0 & Grid & $36.75 \pm 0.17$ & $13 \pm 1$ & $3 \mathrm{C} 295$ & 22.15 \\
\hline & 1 & Grid & $44.00 \pm 0.13$ & $15 \pm 2$ & $3 \mathrm{C} 295$ & 22.15 \\
\hline & 2 & Grid & $41.92 \pm 0.12$ & $14 \pm 2$ & $3 \mathrm{C} 295$ & 22.15 \\
\hline & 3 & Grid & $43.52 \pm 0.13$ & $15 \pm 1$ & 3C295 & 22.15 \\
\hline & 4 & Grid & $44.70 \pm 0.13$ & $16 \pm 2$ & 3C295 & 22.15 \\
\hline & 5 & Grid & $47.91 \pm 0.13$ & $16 \pm 2$ & 3C295 & 22.15 \\
\hline & 6 & Grid & $49.18 \pm 0.17$ & $16 \pm 2$ & $3 \mathrm{C} 295$ & 22.15 \\
\hline \multicolumn{7}{|c|}{ GBT16B_400_13 } \\
\hline & 0 & Grid & $35.22 \pm 0.22$ & $11 \pm 1$ & 3C295 & 22.15 \\
\hline & 1 & Grid & $36.53 \pm 0.23$ & $12 \pm 3$ & $3 \mathrm{C} 295$ & 22.15 \\
\hline & 2 & Grid & $39.52 \pm 0.20$ & $12 \pm 1$ & 3C295 & 22.15 \\
\hline & 3 & Grid & $37.52 \pm 0.33$ & $13 \pm 1$ & 3C295 & 22.15 \\
\hline & 4 & Grid & $39.08 \pm 0.13$ & $13 \pm 1$ & 3C295 & 22.15 \\
\hline & 5 & Grid & $39.60 \pm 0.17$ & $13 \pm 1$ & $3 \mathrm{C} 295$ & 22.15 \\
\hline & 6 & Grid & $38.27 \pm 0.18$ & $13 \pm 1$ & $3 \mathrm{C} 295$ & 22.15 \\
\hline \multicolumn{7}{|c|}{ GBT16B_400_14 } \\
\hline & 0 & Grid & $37.50 \pm 0.33$ & $11 \pm 1$ & $3 \mathrm{C} 147$ & 21.25 \\
\hline & 1 & Grid & $36.67 \pm 0.25$ & $12 \pm 1$ & $3 \mathrm{C} 147$ & 21.25 \\
\hline & 2 & Grid & $39.52 \pm 0.20$ & $11 \pm 1$ & $3 \mathrm{C} 147$ & 21.25 \\
\hline & 3 & Grid & $40.85 \pm 0.22$ & $13 \pm 1$ & $3 \mathrm{C} 147$ & 21.25 \\
\hline & 4 & Grid & $39.35 \pm 0.18$ & $14 \pm 2$ & $3 \mathrm{C} 147$ & 21.25 \\
\hline & 5 & Grid & $47.05 \pm 0.85$ & $13 \pm 1$ & $3 \mathrm{C} 147$ & 21.25 \\
\hline & 6 & Grid & $44.22 \pm 0.47$ & $12 \pm 1$ & $3 \mathrm{C} 147$ & 21.25 \\
\hline
\end{tabular}

Table 1.4 Summary of derived system properties in XX Polarization from calibration scans used to make the science images

The $T_{\text {sys }} / \eta$ for the XX polarization in the boresight beam has relatively little scatter around the mean of $35.7 \pm 0.8 \mathrm{~K}$, while the YY polarization values are consistently higher with a mean value of $40 \pm 1 \mathrm{~K}$ with the highest values coming at the beginning of observing runs. The increase towards higher and lower frequencies is the effect of hitting the analog filter roll-off, resulting in the overall shape of a second order polynomial. The slight increase in YY values are due to several factors such as known dead LNAs, a more favorable backend setup (the first few sessions only ran with a single HPC), calibration grids that include more (and evenly spaced) reference scans (calibration grids for GBT16B_400_1 to GBT16B_400_9 only included two reference scans), and/or better bit and byte lock. The trends for $T_{\text {sys }} / \eta$ presented in this plot is representative for the outer beams. The time averaged $T_{\text {sys }} / \eta$ 


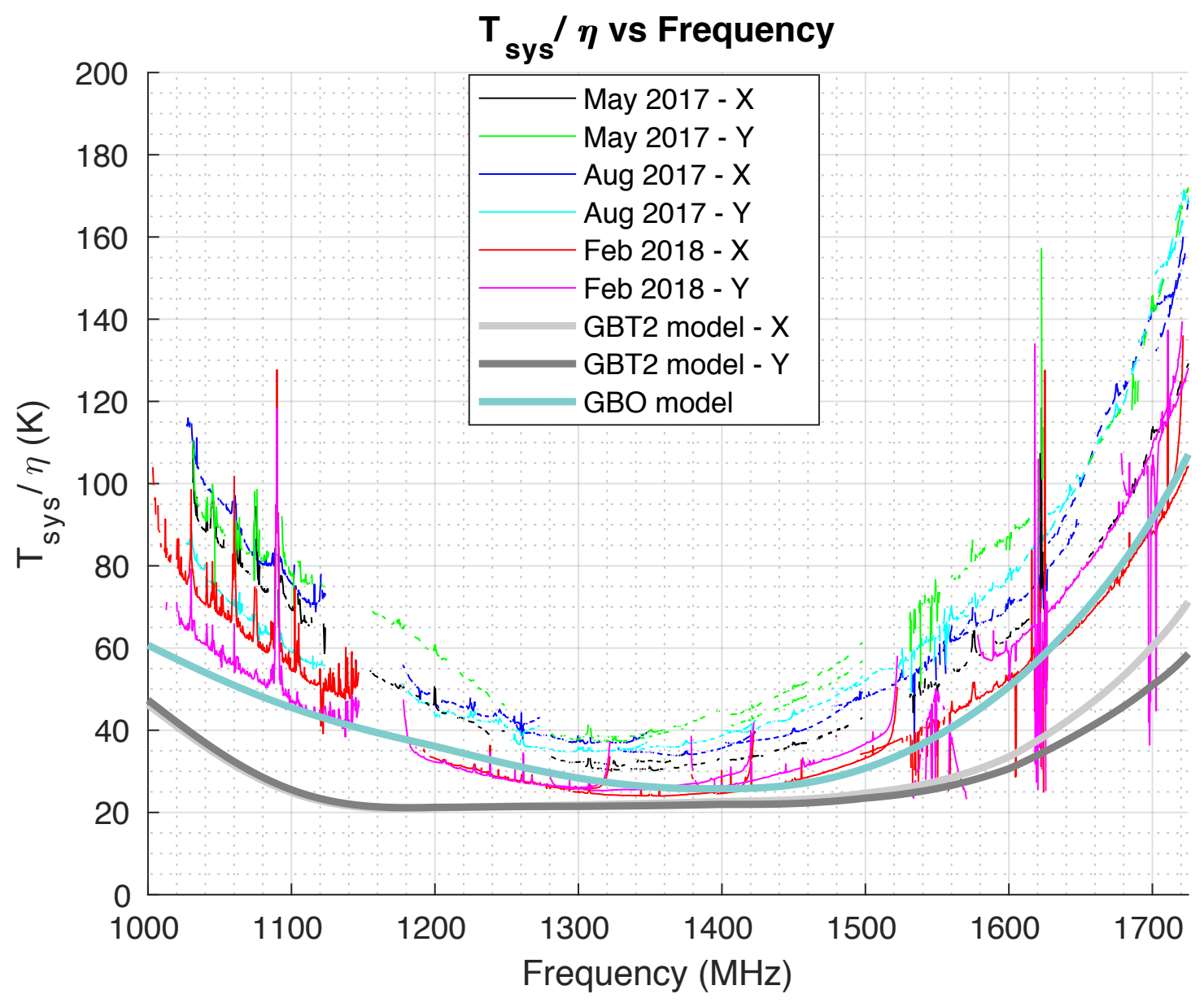

Figure 1.7 Temporal variation of $T_{\text {sys }} / \eta$ for the boresight beam over the entire analog frequency range. The general trend of decreasing $T_{\text {sys }} / \eta$ is also true for all outer beams.

for each beam and polarization are shown in Table 1.3.

The dashed black line horizontal line at $27.7 \mathrm{~K}$ represents the $T_{\text {sys }} / \eta$ of the existing L-Band receiver on the GBT, assuming a $T_{\text {sys }}$ value of $18 \mathrm{~K}$ and $\eta=0.65$ (Boothroyd et al., 2011). While most of the $T_{\text {sys }} / \eta$ lie above this benchmark for the boresight beam, several seven-point and grid calibration observations are at or exceed single pixel performance. Again, this behavior is also true for the outer beams.

Figure 1.7 shows the decrease in $T_{\text {sys }} / \eta$ derived from 'frequency sweep' observa- 
tions of a calibration source. The observing procedure was to perform a seven-point calibration scan with the LO set to $50 \mathrm{MHz}$ increments beginning at $1100 \mathrm{MHz}$ and continuing up to $1700 \mathrm{MHz}$, measure the $T_{\text {sys }} / \eta$ quantity as a function of the $150 \mathrm{MHz}$ bandpass for each formed beam, and merge the results. There is a trend of decreasing $T_{\text {sys }} / \eta$ as a function of time for both polarizations with the February 2018 calibration data showing the lowest observed $T_{\text {sys }} / \eta$, which can be attributed to improvements made to the signal processing algorithms of the backend. Specifically, a bit underflow when the data in the $\mathrm{ROACH}$ is reduced to 8-bit/8-bit real and imaginary values just before packetization.

A correction to increase the digital gain just prior to the re-quantization was implemented for the August 2017 and February 2018 observing runs. For some frequencies, namely $1200 \mathrm{MHz}$ to $1400 \mathrm{MHz}$, the derived $T_{\text {sys }} / \eta$ even out performs the GBO model in Figure 1.7. While this figure again only shows data for the boresight beam, the trend is observed in all outer beams.

A figure of merit to compare the performance of FLAG to the existing singlepixel feed is the respective noise from a single DecLatMap procedure. As discussed in Section 4.4.1, a typical science map is $4 \operatorname{deg}^{2}$ centered on the source and consists of 41 total columns that span $2^{\circ}$ made up of 71 distinct integrations dumped every 1.67'. Consider an integration time of the single-pixel map $\left(t_{i n t, S P}\right)$ set at $5 \mathrm{~s}$ as compared to $0.5 \mathrm{~s}$ for an default FLAG observation. Thus, the rate at which the telescope moves is 0.33 arcminutes $\mathrm{s}^{-1}$ and 3.33 arcminutes $\mathrm{s}^{-1}$ for a single-pixel and FLAG map, respectively. The area of the single-pixel GBT beam with a fullwidth half-max $(\mathrm{FWHM})$ of $9.1^{\prime}$ is $1.1331 \times \mathrm{FWHM}^{2} \sim 94 \operatorname{arcminutes}^{2}$. A standard 
DecLatMap will therefore have 154 GBT beams. Excluding overhead, a single pixel map takes a total of $t_{m a p, S P}=71 \times 41 \times t_{i n t, s p}=14555.0 \mathrm{~s}=4.04$ hours, while an equivalent FLAG map takes $t_{m a p, F L A G}=1455.5 \mathrm{~s}=0.404$ hours. Dividing the total time for the two maps by the number of beams gives a total 'signal' integration time $\left(t_{\text {sig }}\right)$ of $95.1 \mathrm{~s} /$ beam and $9.5 \mathrm{~s} /$ beam for the single-pixel and FLAG, respectively. Recall that spectral line mapping data are calibrated by using the first and last four integrations of particular scan. The effective integration time for the single pixel map is then

$$
t_{\mathrm{eff}}=\frac{t_{\mathrm{sig}} t_{\mathrm{ref}}}{t_{\mathrm{sig}}+t_{\mathrm{ref}}}=\frac{95.1 \cdot 40}{95.1+40}=28.2 \mathrm{~s} / \text { beam } .
$$

Repeating the calculation for the equivalent FLAG map gives $2.8 \mathrm{~s} /$ beam. The factor of 10 decrease is due to $t_{\text {int,FLAG }}$ being set to $0.5 \mathrm{~s}$ as opposed to $5 \mathrm{~s}$; thus, $t_{\text {map,FLAG }}, t_{\text {ref }}$, and $t_{\text {sig }}$ are similarly a factor of 10 lower. This is not to say that a single equivalent FLAG map will reach the same sensitivity in $10 \times$ less time.

The predicted root-mean-square (rms; $\sigma$ ) is given by the ideal radiometer equation in Equation 1.12. Recall that only the XX polarization is analyzed, so $N_{\text {pol }}$ is equal to one), $\Delta \nu$ represents the frequency width of a single velocity channel (typically $24.414 \mathrm{kHz}$ ). In our case, the equivalent system flux density derived from Equation 4.9 can be directly substituted for $T_{\text {sys }}$ in the equation above to calculate the theoretical rms noise in units of Jy/beam. Utilizing the antenna of gain of 1.86 $\mathrm{K} / \mathrm{Jy}$ derived in Section 2.3.1 and assuming a $T_{\text {sys }}$ of $18 \mathrm{~K}$, the equivalent $S_{\mathrm{SEFD}}$ for a typical single pixel observation is $9.68 \mathrm{Jy} /$ beam. Plugging in the respective $t_{\text {eff }}$ values for a typical the single-pixel and FLAG map, and taking the mean $S_{\text {SEFD }}$ 
of the boresight beam used to scale all science observations in Table 1.4, gives the theoretical rms respectively as $12 \mathrm{mJy} /$ beam versus $36 \mathrm{mJy} /$ beam for a single polarization in the single pixel and and single formed beam in the FLAG maps, respectively. Assuming $36 \mathrm{mJy} /$ beam for the other six electronically formed beams in a FLAG map, the predicted FLAG noise should drop by a factor of $1 / \sqrt{7}$ to be $13.6 \mathrm{mJy} /$ beam, which compares reasonably well to an equivalent single-pixel map. There is, of course, inherent scatter in $S_{\mathrm{SEFD}}$. A more exact calculation for the noise in a single FLAG map is

$$
\sigma=\frac{1}{\sqrt{N \cdot N_{\mathrm{pol}} \Delta \nu t_{\mathrm{eff}}}} \sum_{\mathrm{i}=1}^{N} S_{\mathrm{SEFD}, \mathrm{i}}
$$

where $N$ is the total number of beams (usually seven, in our case). Plugging in the mean $S_{\mathrm{SEFD}}$ for each beam as shown in Table 1.4 and again considering only a single polarization, the typical rms noise for a typical FLAG map will be $55 \mathrm{mJy} /$ beam. Scaling the $t_{\text {eff }}$ of $2.82 \mathrm{~s} /$ beam for a single electronically formed FLAG beam by a factor of 7 to account for all formed beams, and taking the ratio of the result with the single-pixel $t_{\text {eff }}$ of $28.20 \mathrm{~s} /$ beam gives 1.4 . This ratio implies an observer can reach an equivalent $t_{\text {eff }}$ in $1.4 \times t_{\text {map,FLAG }}$, or 0.57 hours. Thus, the total mapping time required to reach an equivalent single pixel $t_{\text {eff }}$ reduces by a factor of $t_{\text {map,SP }} / 0.57$ $=4.04 / 0.57 \sim 7$ 

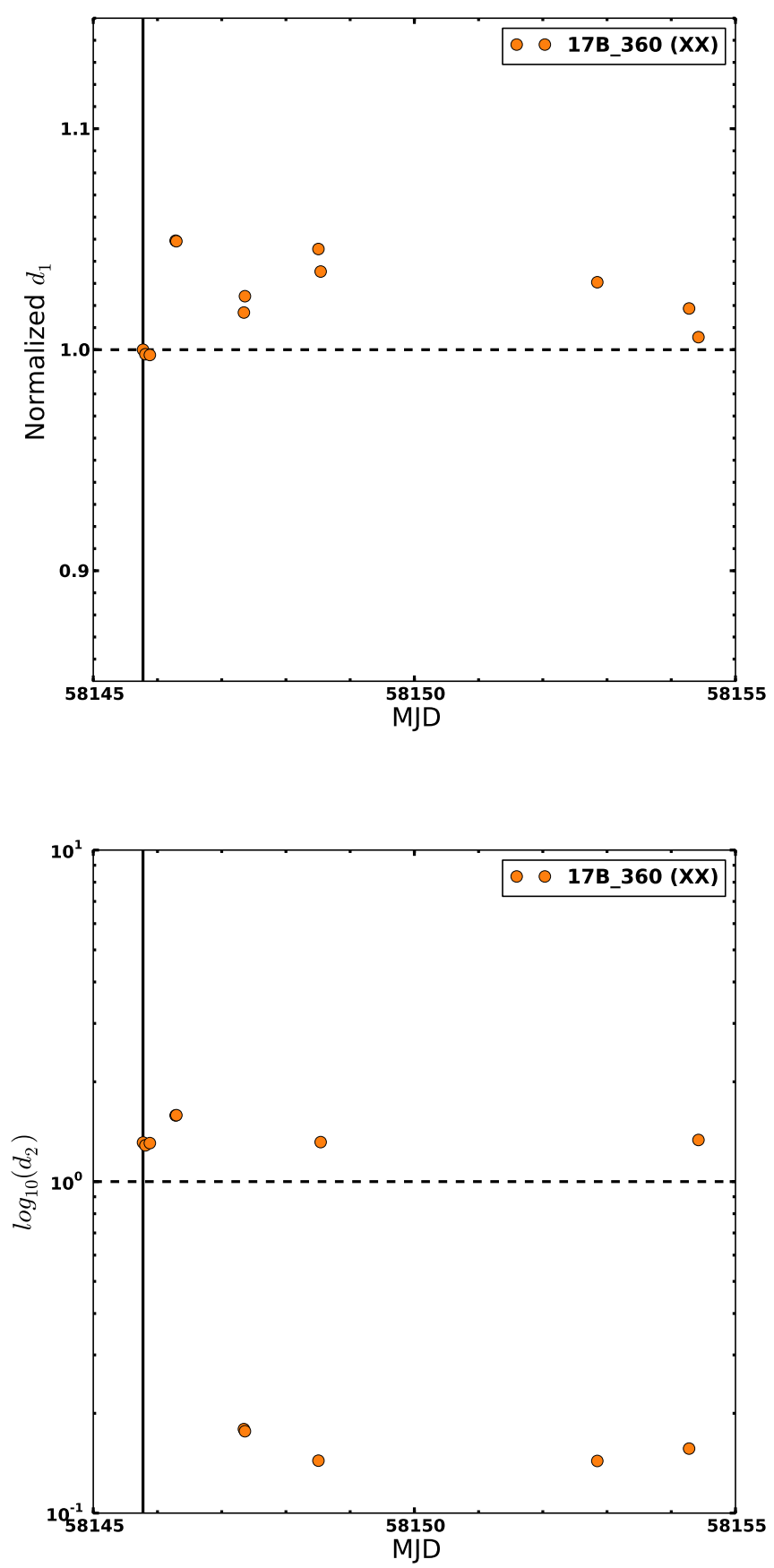

Figure 1.8 Normalized $d_{1}$ (see text; top) and $d_{2}$ (bottom) between the first and subsequent weight calibration vectors for GBT17B_360 sessions 01 to 07, GBT17B_455 session 01, and GBT18A_443 session 01 (orange). All values shown are for the boresight beam in XX polarization, though very similar trends exist for the outer beams. The horizontal dashed lines show where these quantities would indicate perfect agreement over time, while the solid vertical line denotes the beginning MJD of the observing run. 


\subsubsection{Beamformer Weight Validity over Time}

The calibration procedures described above, while straightforward and not too time consuming, still contribute to about 30 minutes of overhead and, in principle, are only valid for a given 'word/bit lock'. The front end employs a new digitaldown-link mechanism that performs all analog-to-digital conversions in a compact assembly that sits at prime focus (Morgan et al., 2013). Two integral processes in the success of the DDL are achieving word and bit lock, which respectively refer to the recovery of the most-significant bit from each input sample and the deserialization bit alignment along the optical fiber. Since word/bit lock is currently lost with every change in the LO setting, an observer must derive fresh beam weights at the beginning of each observing session to achieve any formed beams on the sky and maintain an advantage over a traditional single pixel feed. Other reasons recalibration may be necessary include large variations in the contribution of spillover and sky noise to the signal model and the relative electronic gain drift between dipoles (Jeffs et al., 2008). Important factors that impact the quality of the weights include robust word and bit locks, constraining the desired steering vector for a formed beam, and utilizing a sufficiently bright calibration source to adequately characterize the system response when on and off source.

While the current state of the FLAG system requires new beamforming weights every session, it is still interesting to explore how the complex weight vectors derived from a given calibration observation vary with time between sessions and even different calibration scans within the same session. Studying the variations will help 
reveal characteristic properties and/or behavior of the weights that demonstrate the stability of the system with time.

Recall that an element in the weight vector as described by Equation 4.2 is a complex number that contains the amplitude and phase information to be applied to the output of a given dipole in order to steer a beam in a given direction. It is therefore sensible to compare these two quantities to obtain a qualitative description of these weights over time. Dramatic variations in the phase indicate that the two weight vectors being compared will not provide reliably similar on-sky beam patterns, since the directional response to an incoming signal originating from the same incidence angle will be different. To measure the difference in phase, a distance metric can be defined

$$
d_{1}=\left\|\mathbf{\mathbf { a } _ { 1 }}-\tilde{\mathbf{\mathbf { a } _ { 2 }}}\right\|
$$

where $\mathbf{a}_{\mathbf{1}}$ and $\mathbf{a}_{\mathbf{2}}$ are the vector norms (i.e., the square root of the sum of each element's squared complex modulus) of the weight vectors, or $\mathbf{w}_{\mathbf{1}} /\left\|\mathbf{w}_{\mathbf{1}}\right\|$ and $\mathbf{w}_{\mathbf{2}} /\left\|\mathbf{w}_{\mathbf{2}}\right\|$, respectively.

The vector $\tilde{\mathbf{a}_{2}}$ represents the subsequent weight vector that has been corrected for the 'bulk phase difference' between the two vectors. This bulk phase offset arises from the steering vectors, which are found by solving for the dominant eigenvector in the generalized eigenvalue problem in Equation 4.7. Since eigenvectors are basis vectors that have arbitrary scaling, it is the unknown scaling of the phase between calibration data sets that contributes to the bulk phase offset. A subsequent weight vector can be phase aligned to some initial weight vector by first making the first 
element of $\mathbf{a}_{\mathbf{1}}$ real and then computing

$$
\hat{\phi}=\angle\left(\mathbf{a}_{\mathbf{2}}^{\mathbf{H}} \mathbf{a}_{\mathbf{1}}\right)
$$

where $\hat{\phi}$ is the angle of the Hermition product of $\mathbf{a}_{\mathbf{2}}^{\mathbf{H}} \mathbf{a}_{\mathbf{1}}$. The phase aligned weight vector is therefore $\tilde{\mathbf{a}_{2}}=e^{i \hat{\phi}} \mathbf{a}_{\mathbf{2}}$.

Because the distance metric $d_{1}$ is the overall magnitude of an element-wise difference between two an M element vectors, it encapsulates all the phase differences between respective dipoles into a single quantity. Small variations in $d_{1}$ over time indicate similar phases (save for the bulk phase offset due, in part, to new word/bit lock) between the derived weight vectors, meaning the direction response of the array is stable over time; thus, the beam pattern shape and $T_{\text {sys }} / \eta$ remain relatively unchanged.

The two vectors used to compute $d_{1}$ are unit normalized, so it does not describe the scale variations between two weight vectors. Large variations in the relative scale will affect the gain stability over the field-of-view, and may cause difficulties in flux calibrations between observing sessions. To detail this behavior a separate distance metric can be defined

$$
d_{2}=\left\|\mathbf{w}_{\mathbf{2}}\right\| /\left\|\mathbf{w}_{\mathbf{1}}\right\|
$$

Figure 1.8 shows the $d_{1}$ (top) and $d_{2}$ (bottom) as a function of time for the 16B_400 run consisting of sessions 09 to 14 (blue) and 17B_360 run consisting of sessions 01 to 07 (blue) and calibration data from 17B_455_01 (orange) and 18A_443_01 
(orange). Note that $d_{1}$ is normalized to the value computed between the first weight vector derived for a particular observing run and the subsequent weight vector from the next calibration observation to represent a fractional change. A value of unity for both quantities indicates no variation in the weight vectors over time. Only mild variation $(\lesssim 5 \%)$ is seen in the distance metrics tracing the total change in phase over time, indicating that the directional response to identical coincident signals is very similar over time. However, the same cannot be concluded for the scale variations as traced by $d_{2}$. For several sessions in the 17B_360 the amplitude fluctuates between factors of 20 to 40, although the weight vectors derived for 16B_400 are relatively stable. While not affecting overall sensitivity, such large amplitude variations introduce significant difficulties in the process of matching flux scales between observations. This is especially arduous when multiple observing sessions of the same object are needed to be combined. If such behavior can be determined during an observing session, it would be prudent of the observer to consider re-deriving the weight vector to acquire a more consistent amplitude.

\subsubsection{Hi FLAG Observations}

Two key milestones for the FLAG system are the detection of HI in an extragalactic source and the completely recovery of the Hi emission as already traced by the single pixel feed. This subsection will therefore focus on the results of observations of two sources that have ample existing GBT data for comparisons of the noise, spatial and spectral coincidence, and overall flux scaling. 


\subsubsection{NGC6946}

The HI distribution in NGC6946 is well studied utilizing both high angular resolution VLA data (Boomsma et al., 2008) and GBT data. This particular source is an ideal candidate for testing the sensitivity of the FLAG maps given the single pixel GBT data for NGC6946 with an rms noise of $8 \mathrm{mK}$ per $5.2 \mathrm{~km} \mathrm{~s}^{-1}$ velocity channel, or a $3 \sigma N_{\mathrm{HI}}$ detection level of $4.5 \times 10^{18} \mathrm{~cm}^{-2}$ over a $20 \mathrm{~km} \mathrm{~s}^{-1}$ spectral line, and the presence of several companion galaxies in the field (Pisano, 2014). Furthermore, the proximity of the source emission in relation to Milky Way emission and the relatively large angular extent of the emission in and around NGC6946 provide good tests for spatial and spectral coincidence in the beam-combined data (see procedure described in Section 4.4.4).

Figure 1.9 shows the flux as a function of velocity for the single pixel data (orange line) and FLAG data (blue). Each FLAG data cube was regridded to the same FOV and spectral scale as the single pixel data cube to ensure the flux was measured over the exact same angular area. For all instances save for Beam 3, we see that the flux as detected by FLAG is $\sim 30 \%$ lower than what is detected by the single pixel data.

There could be several reasons for such a large flux offset between the two data sets. A particularly conspicuous source for the offset may also lie in the weighted average of the two frequency dithered bandpasses. The flux as a function velocity is again plotted but for the cubes generated from GBT16B_400_12 (LO at 1450.0000 $\mathrm{MHz}$ ) and GBT16B_400_13 (LO at 1449.8484) MHz in Figures 1.10 and 1.11, re- 


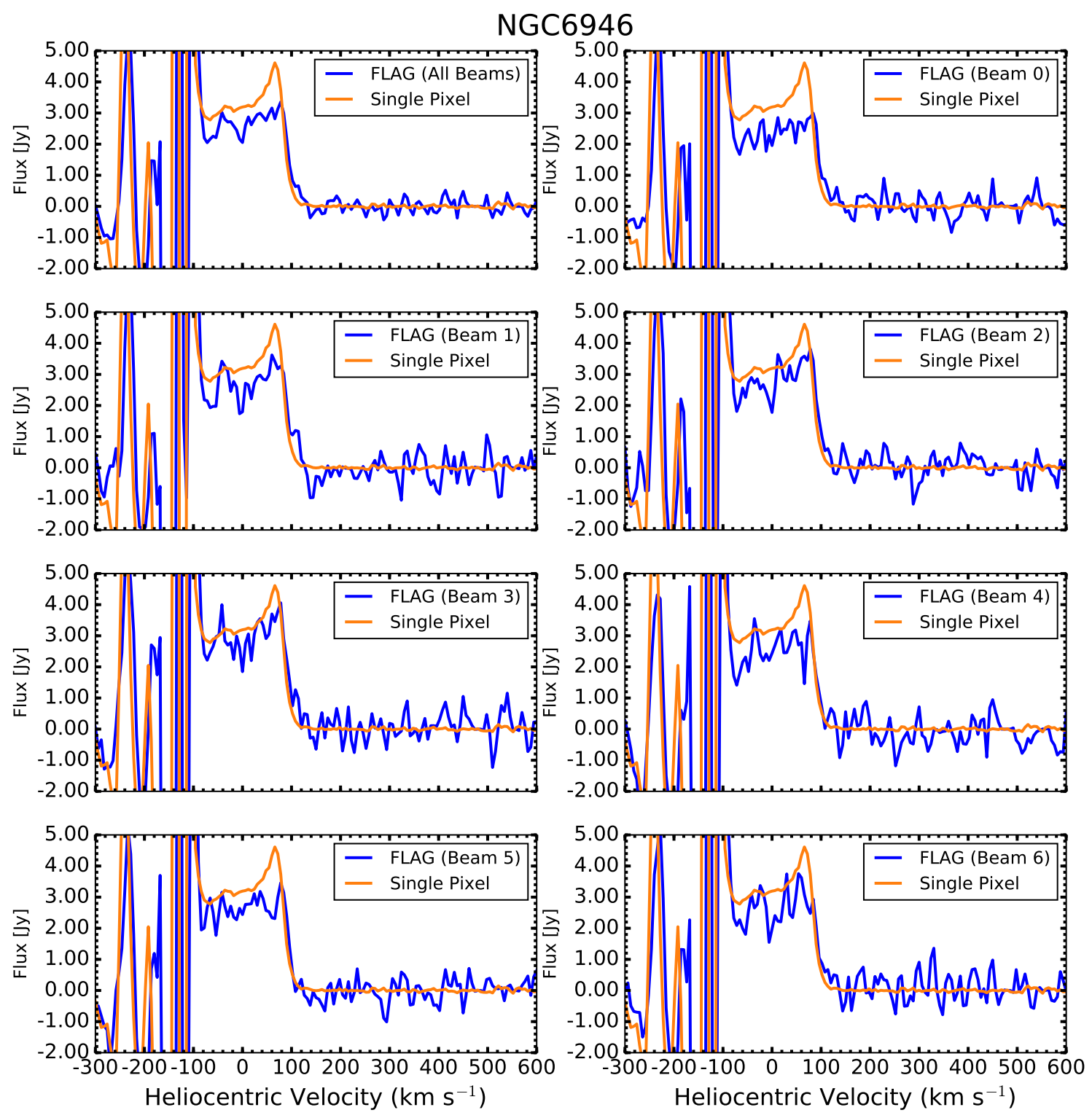

Figure $1.9 \mathrm{HI}$ profiles showing flux as a function of velocity for the combined data set (all beams; top left), in addition to the seven individual beams. All data are from the weighted average combination of the frequency dithered observations from sessions GBT16B_400_12 (LO set to $1450.00 \mathrm{MHz}$ ) and GBT16B_400_13 (LO shifted down one-half of a course channel to $1449.85 \mathrm{MHz}$. The data are weighted by the respective $S_{\mathrm{SEFD}}$ for each beam. 

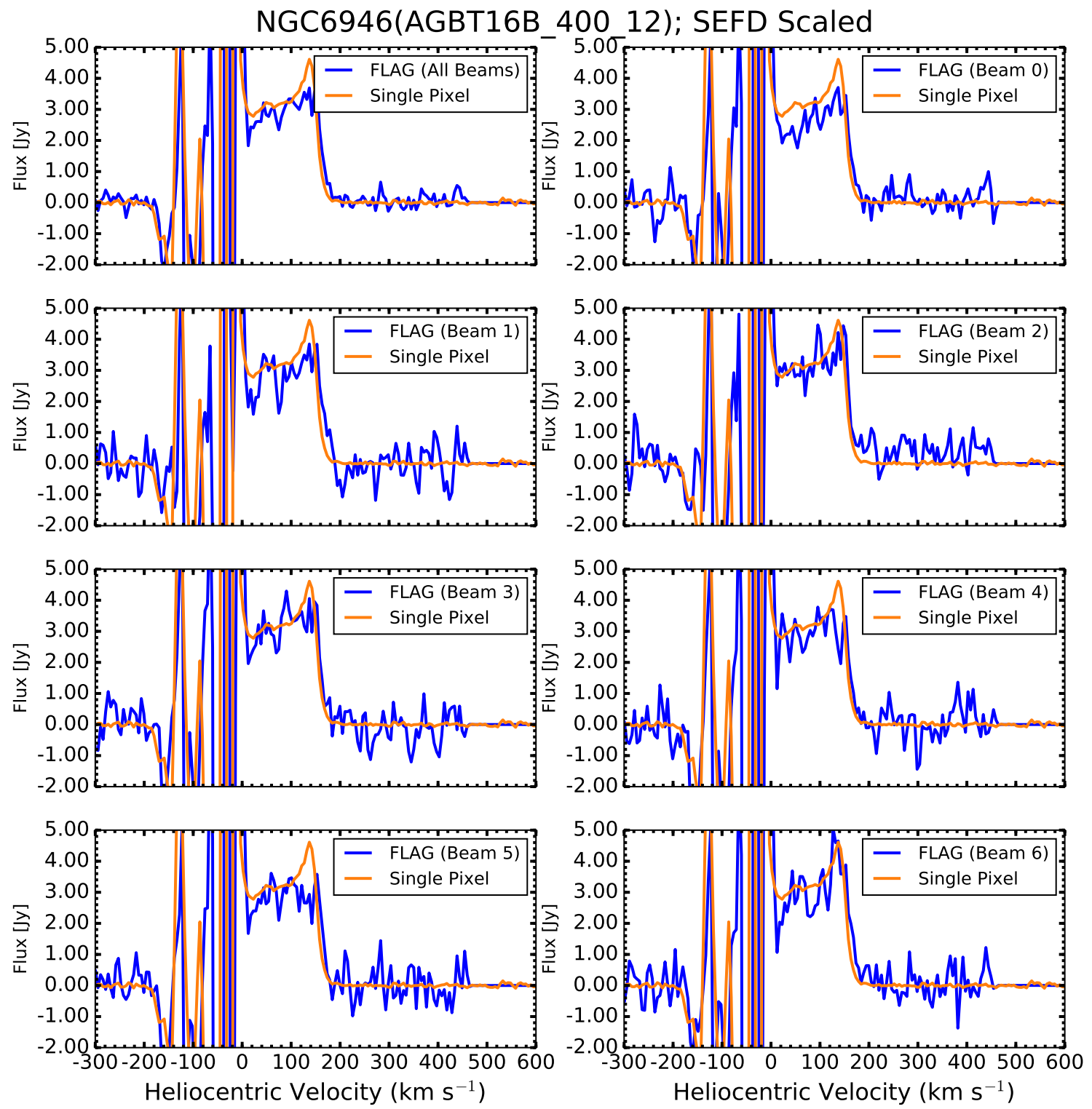

Figure 1.10 Hi profiles showing flux as a function of velocity for the combined data set of GBT16B_400_12 (LO set at $1450.0000 \mathrm{MHz}$ ), in addition to the seven individual beams. All data were scaled by the $S_{\mathrm{SEFD}}$ for each beam. 
NGC6946 (AGBT16B_400_13); SEFD Scaled
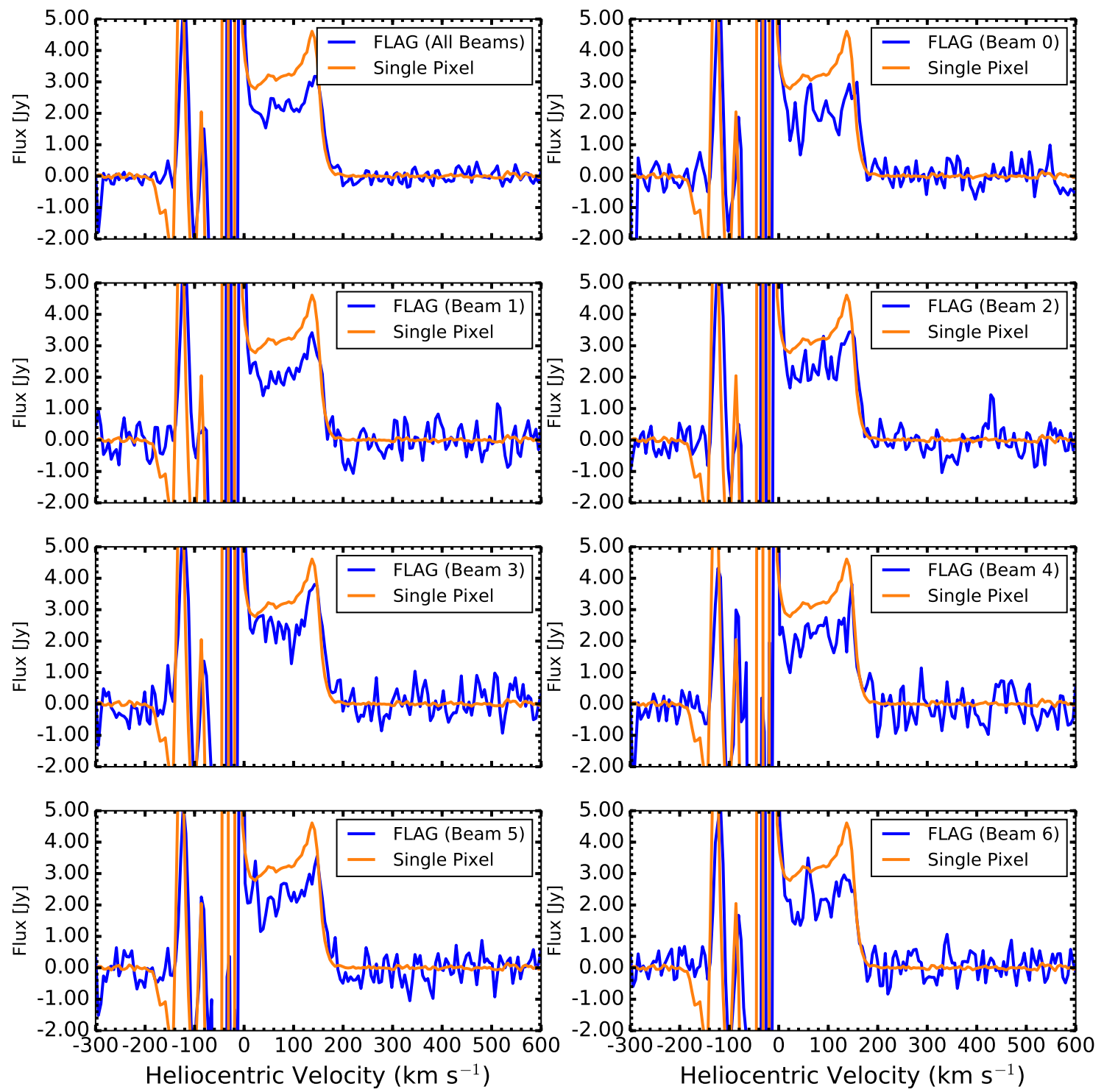

Figure 1.11 Hi profiles showing flux as a function of velocity for the combined data set of GBT16B_400_13 (LO set at $1449.8484 \mathrm{MHz}$ ), in addition to the seven individual beams. All data were scaled by the $S_{\mathrm{SEFD}}$ for each beam. 

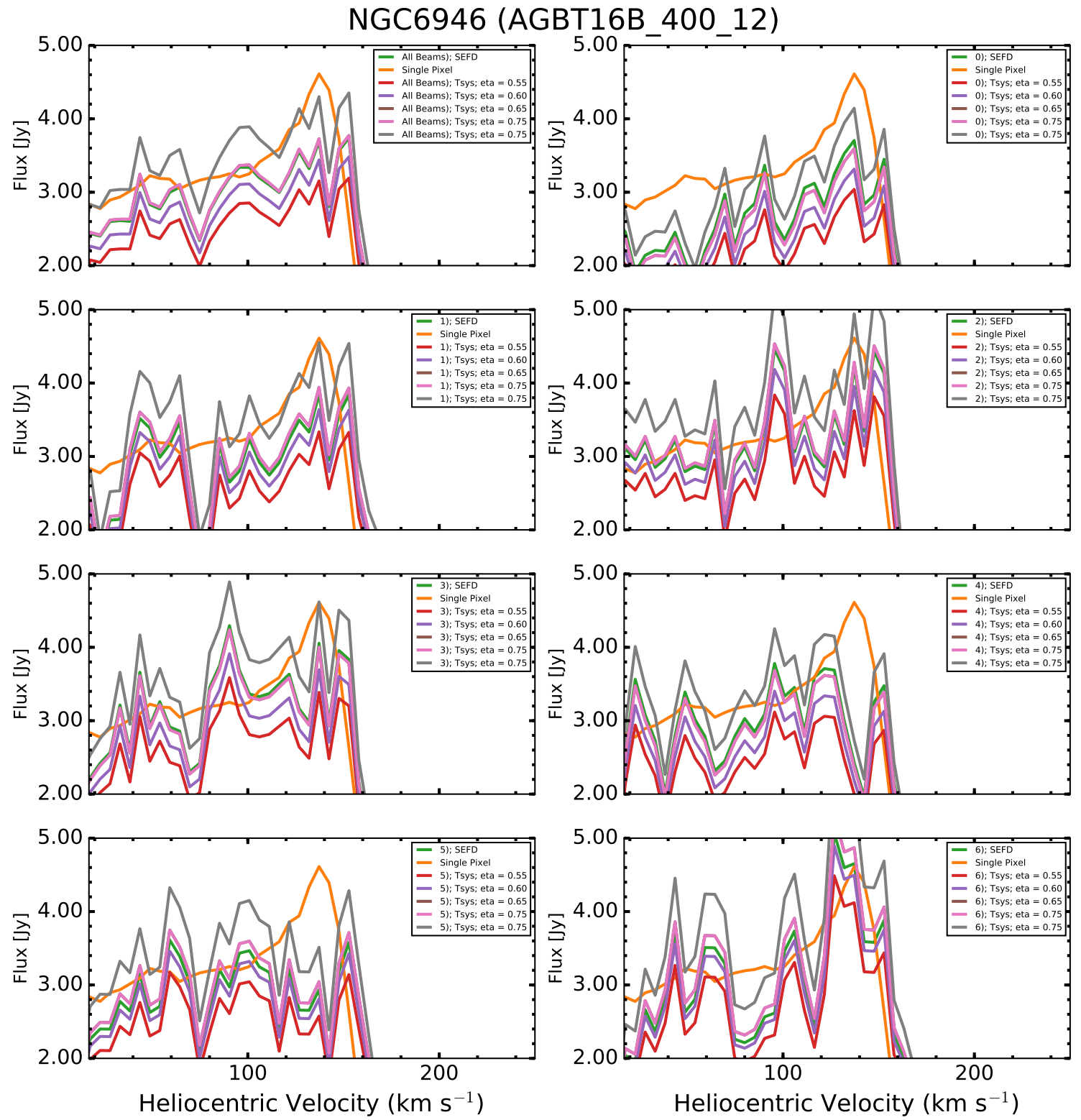

Figure 1.12 Hi profiles showing flux as a function of velocity for the data set of GBT16B_400_12 where the calibrated data were scaled by the derived $S_{\text {SEFD }}$ (green) and $T_{\text {sys }} / \eta$ varying $\eta$ from 0.55 (red), 0.60 (purple), 0.65 (magenta), 0.70 (pink), and 0.75 (grey). The single pixel profile is denoted in orange. 

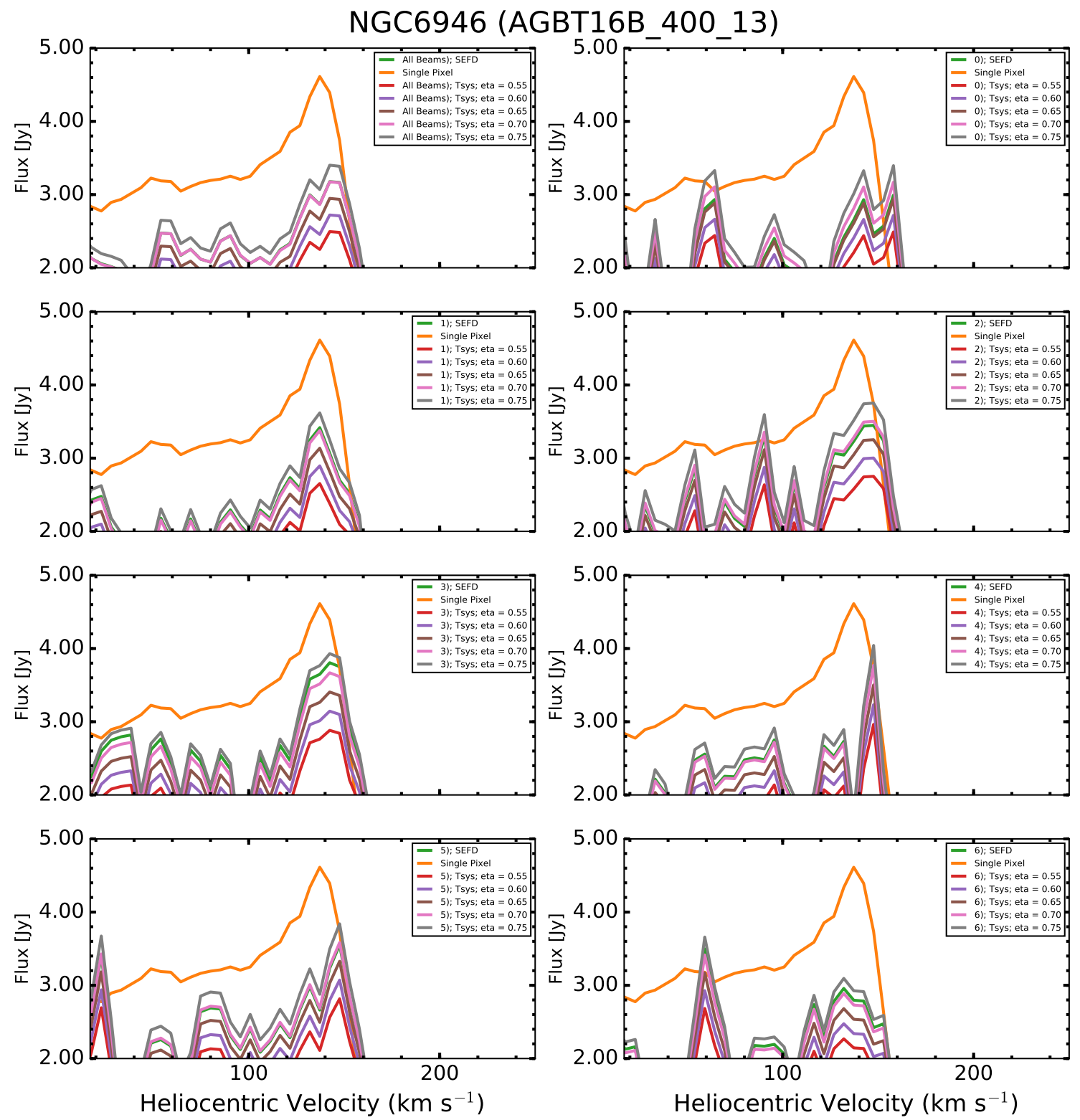

Figure $1.13 \mathrm{HI}$ profiles showing flux as a function of velocity for the combined data set of GBT16B_400_13 where the calibrated data were scaled by the derived $S_{\text {sys }}$ (green) and $T_{\text {sys }} / \eta$ varying $\eta$ from 0.55 (red), 0.60 (purple), 0.65 (magenta), 0.70 (pink), and 0.75 (grey). The single pixel profile is denoted in orange. 

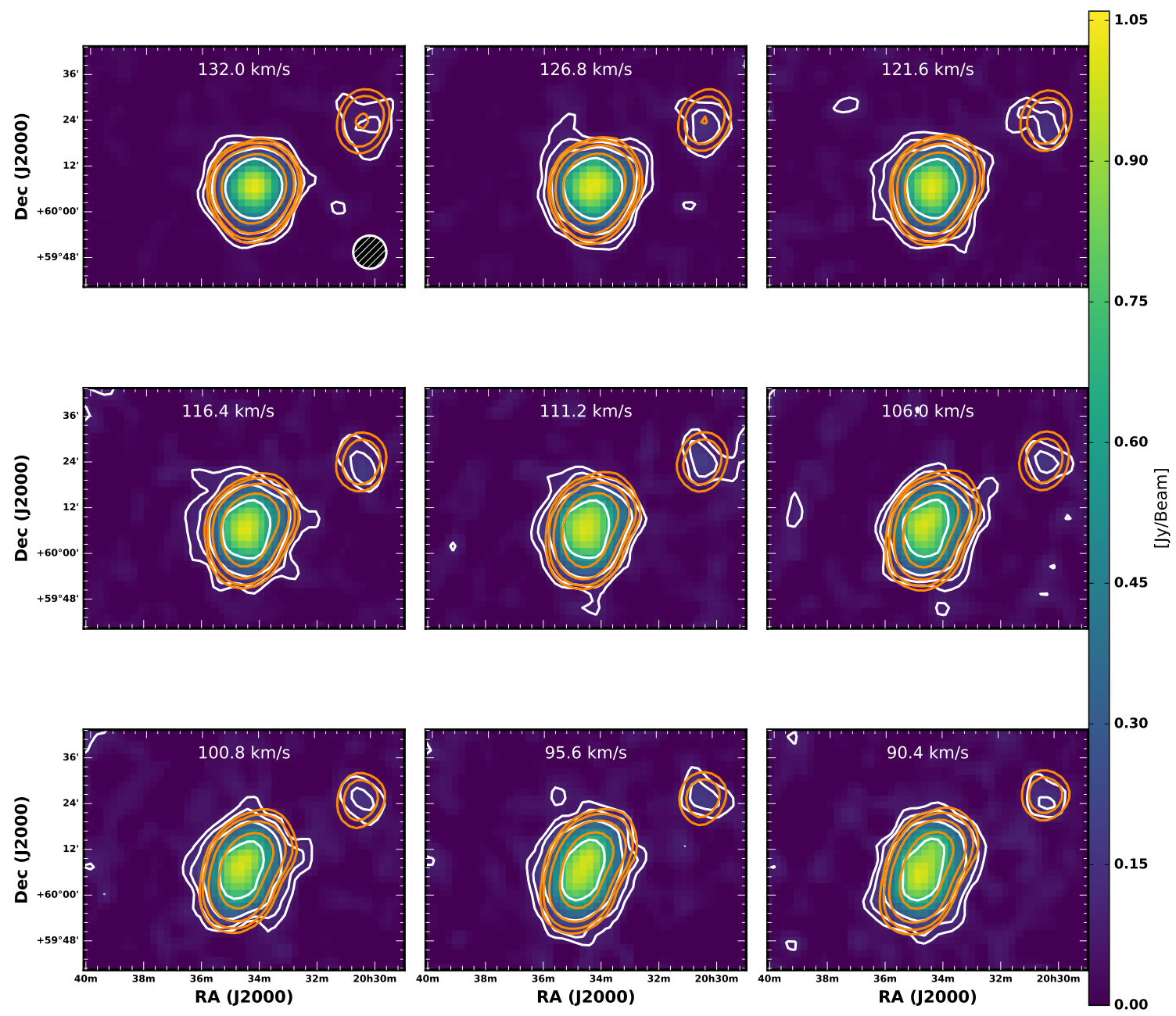

Figure 1.14 Channel maps of the combined data of NGC6946 (all beams from session 12 and 13). In all panels, the color scale and white contours represent the FLAG data, while the orange contours are the single pixel counterpart data set; both sets of contours begin at the 3 times the rms noise of the FLAG data (20 mJy/beam) and continue at 5, 10, and 25 times that level. 


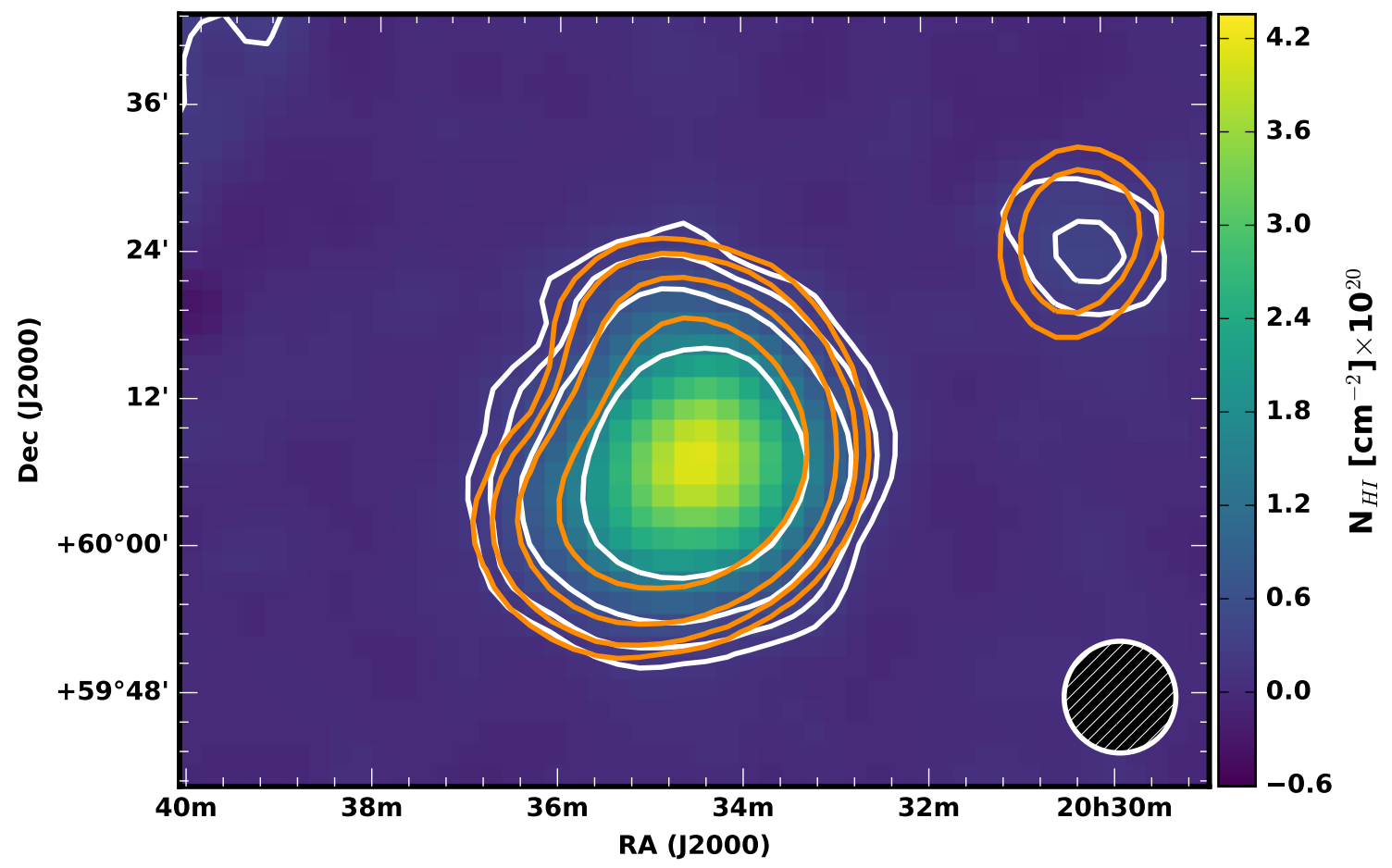

Figure 1.15 $N_{\mathrm{HI}}$ map of NGC6946. Again, the color scale and white contours represent the FLAG data, while the orange contours are the single pixel counterpart data set; both sets of contours begin at the -3 times the $1 \sigma$ uncertainty for the integrated FLAG data set $\left(7 \times 10^{18} \mathrm{~cm}^{-2}\right.$; dashed lines) of the FLAG data and continue to 3 , 5,10 , and 25 times that level (solid lines). 
spectively. There is a noticeable discrepancy between the flux profiles of the two data sets and single pixel data. For example, the percent error (taking the singlepixel flux as a 'theoretical' value of comparison) in the total flux measured between the velocities of $18 \mathrm{~km} \mathrm{~s}^{-1}$ and $210 \mathrm{~km} \mathrm{~s}^{-1}$ (to avoid the Galactic HI) for the combined beam data is $(5.5 \pm 0.4) \%$ for the GBT16B_400_12 in Figure 1.10, as opposed to $(29 \pm 2) \%$ for the equivalent cube for GBT16B_400_13 in Figure 1.11. Scale variations in the beamformer weights (see bottom panel of Figure 1.8) could contribute to the large offset. Given the very similar $S_{\mathrm{SEFD}}$ for each beam between the two sessions (See Table 1.4), using the inverse square of of the respective $S_{\mathrm{SEFD}}$ the weights in the weighted average between the two data sets will inevitably result in a final combined flux profile that is well below that of single pixel profile.

In an attempt to better match the single pixel profile, additional cubes were generated that were scaled with the derived $T_{\text {sys }} / \eta$ (see column 3 of Table 1.4) instead of $S_{\mathrm{SEFD}}$, but assuming varies values of $\eta$ set at $0.55,0.60,0.65,0.70$, and 0.75 . Zoom-ins of flux profiles that highlight the overall changes are shown in Figures 1.12 and 1.13. In the case of the GBT16B_400_12 data, the profile corresponding to $\eta$ equal to 0.75 initially looks to best matches the single pixel profile for all beams. However, when inspecting the integrated flux, the lowest percent error consistently measured over each beam is with the data scaled originally by the derived SEFD.

A large flux discrepancy still exists for the GBT16B_400_13 profiles in Figure 1.13 regardless of assumed $\eta$. In order to weight the data in a manner that reflects the more consistent flux scaling of the GBT16B_400_12 profiles as compared to the reference single pixel profile, the weight coefficients in the weighted average 
of the two data sets are the inverse squares of the percent error in total flux measured between $18 \mathrm{~km} \mathrm{~s}^{-1}$ and $210 \mathrm{~km} \mathrm{~s}^{-1}$ for the data scaled by the SEFD and single pixel data set (with a total flux of (474 \pm 24$) \mathrm{Jy} \mathrm{km} \mathrm{s}^{-1}$. These percent errors, along with the total flux values detected for each beam from GBT16B_400_12 and GBT16B_400_13 are tabulated in Table 1.5; the mean percent error for the seven beams of GBT16B_400_12 is s ranges between $(5.9 \pm 0.4) \%$, while increasing to a mean percent error of $(28 \pm 1) \%$ between beams for GBT16B_400_13.

The final profiles of these combined data are shown in Figure 1.16. Due to the larger flux offset in the GBT16B_400_13 data, the discrepancies in the final profiles have increased slightly to a mean value of $(12.7 \pm 0.9) \%$.

Despite the flux offset, the spatial and spectral agreement between the emission contours in individual channel maps of the final combined cube as shown in Figure 1.15 is very good in general. Discrepancies arise in the lowest contour level, a $3 \sigma$ noise level of $60 \mathrm{mJy} / \mathrm{beam}$ per channel. This can be attributed to the structure of the sidelobes in the beam-combined cube is very likely complex due to averaging several similar, yet still distinct, aperture illumination patterns as determined by the complex beamformer weights. The agreement along the spectral emission demonstrates that the filler software, PAF_Filler.py, correctly performs the conversion from the topocentric to the heliocentric spectral frame.

The contours in the $N_{\mathrm{HI}}$ image shown in Figure 1.14 also agree well at the high to middle $N_{\mathrm{HI}}$ levels before slightly deviating as in the individual channels maps at the lowest contour level. Again, this structure can be attributed to the complex sidelobe pattern. 


\begin{tabular}{lcc}
\hline \hline Beam $^{\mathrm{a}}$ & Flux $^{\mathrm{b}}[\mathrm{Jy} \mathrm{km} \mathrm{s}$ & \\
\hline GBT16B_400_12 & & Percent Errors \\
Combined $[\%]$ \\
0 (Boresight) & $448 \pm 22$ & $5.5 \pm 0.4$ \\
1 & $394 \pm 20$ & $17 \pm 1$ \\
2 & $425 \pm 21$ & $10.3 \pm 0.7$ \\
3 & $497 \pm 25$ & $4.8 \pm 0.3$ \\
4 & $473 \pm 24$ & $<1$ \\
5 & $394 \pm 22$ & $17 \pm 1$ \\
6 & $431 \pm 22$ & $9.1 \pm 0.6$ \\
GBT16B_400_13 & $475 \pm 24$ & $<1$ \\
Combined & & \\
0 (Boresight) & $339 \pm 17$ & $28 \pm 2$ \\
1 & $310 \pm 16$ & $35 \pm 2$ \\
2 & $317 \pm 16$ & $33 \pm 2$ \\
3 & $367 \pm 18$ & $23 \pm 1$ \\
4 & $397 \pm 20$ & $16 \pm 1$ \\
5 & $322 \pm 16$ & $32 \pm 2$ \\
6 & $347 \pm 17$ & $27 \pm 2$ \\
\hline
\end{tabular}

Table 1.5 Flux Offsets for NGC6946 Data

${ }^{a}$ Formed Beam Number

${ }^{b}$ Total flux in FLAG cube

${ }^{c}$ Percent Error (taking the single-pixel flux of (474 \pm 24$) \mathrm{Jy} \mathrm{km} \mathrm{s}^{-1}$ as the value of comparison 
NGC6946
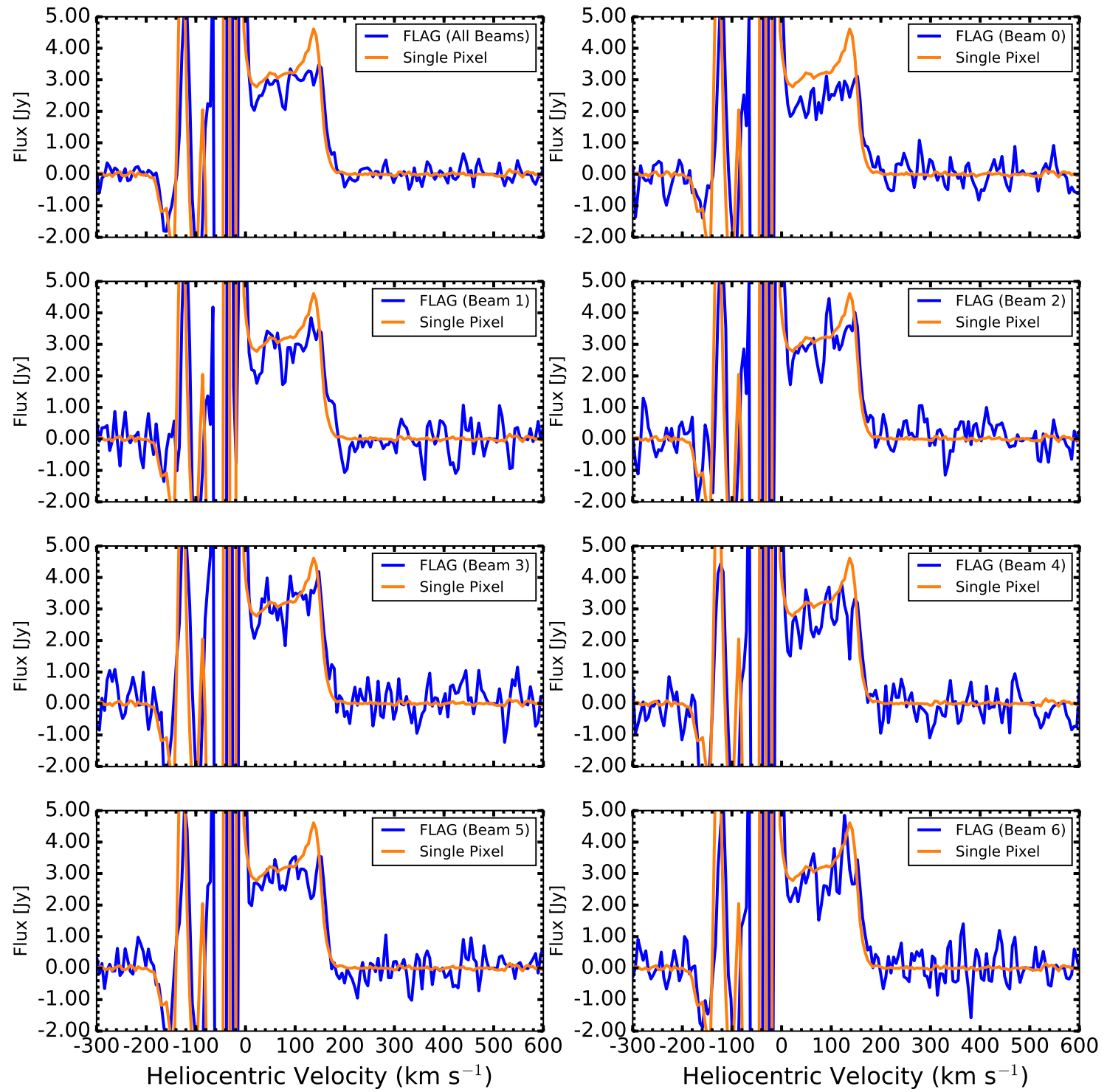

Figure 1.16 Hi profiles showing flux as a function of velocity for the combined data set (all beams; top left), in addition to the seven individual beams. All data are from the combination of the the frequency dithered from GBT16B_400_12/13. 


\subsubsection{M31-M33 Cloud}

The Hi cloud, M31 Cloud 6, is a discrete structure that exists between the Local Group galaxies M31 and M33 at an adopted distance of $800 \mathrm{kpc}$ (Wolfe et al., 2016). This and several other clouds were first observed at low enough angular resolution enough with the WSRT in total power mode Braun \& Thilker (2004) that the they resembled a smooth and extended Hi feature. The high angular resolution, high velocity resolution, and high sensitivity observations of Wolfe et al. (2013) performed with the GBT resolved the HI into discrete clouds approximately the size of a GBT beamwidth. This particular cloud possesses the highest peak brightness temperature of Wolfe et al. (2016) sample at $80 \mathrm{mK}$ and is an excellent source to test how the measured rms goes with $t_{\text {eff }}$ for FLAG.

The observations of M31 Cloud 6 consisted of four total on/off pointing pairs with a duration of 5 minutes for each scan. The last pair of on/off pointings are excluded from the remaining analysis due to RFI present in all integrations. Note that due to time constraints, no dithered frequency observation was performed on this source. Seven total beams were formed with the spacing listed in the first two columns of Table 4.4.1. The pointing data for each formed beam were calibrated according to Equations 4.9 and 1.10 and continuum subtracted from a fit to the emission free regions with a 4th order polynomial. The average noise measured over several emission free channels is $6 \mathrm{mJy} /$ beam, which is within $10 \%$ of the theoretically predicted noise considering a $S_{\mathrm{SEFD}}$ of $12 \mathrm{Jy} /$ beam (see Table 1.4), $\Delta \nu$ of $9.474 \mathrm{kHZ}$, and $t_{\mathrm{eff}}$ of 7.5 minutes. 
M31 Cloud 6
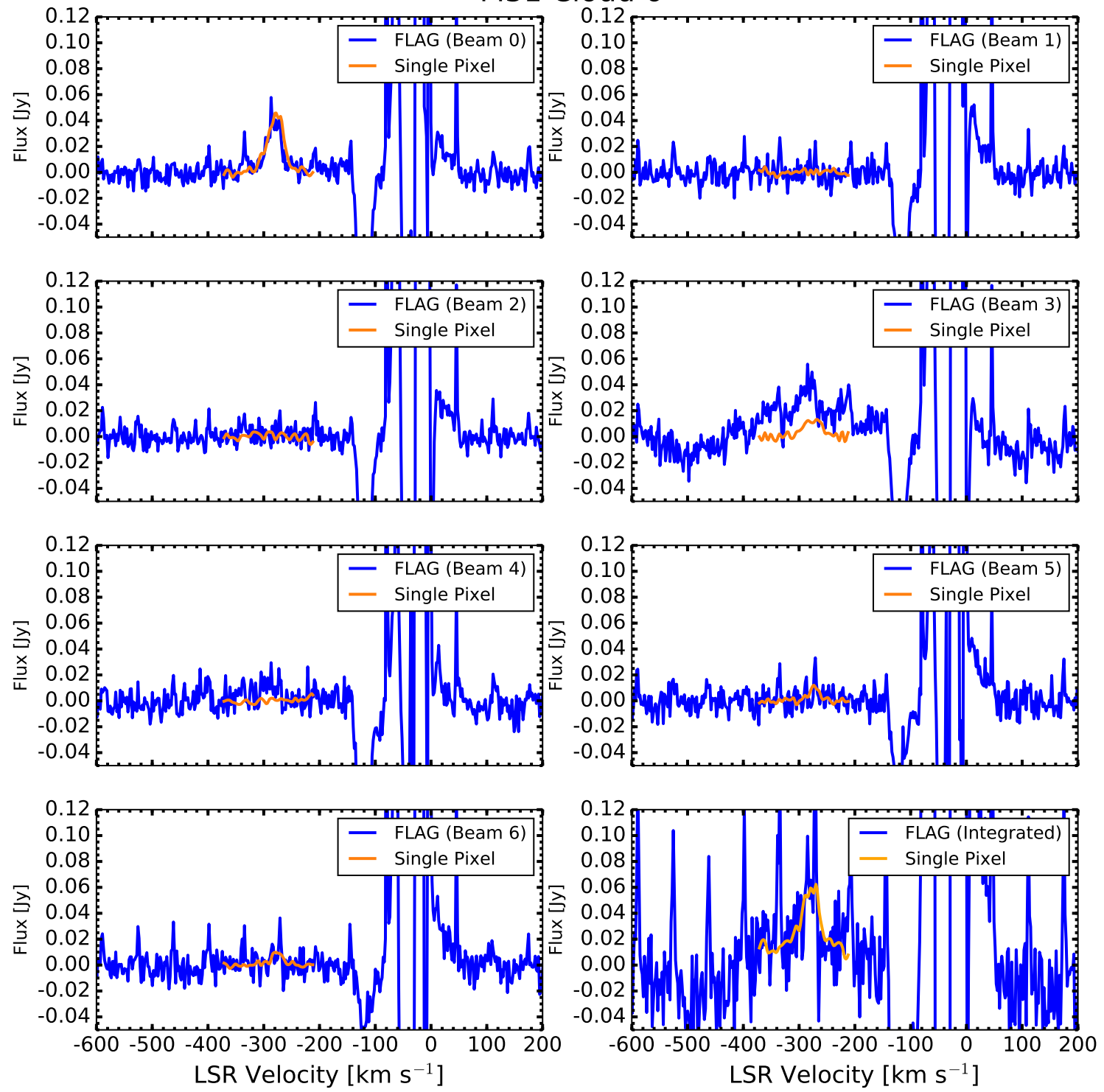

Figure 1.17 Hi profiles showing flux as a function of velocity for the individual beams of the pointing towards M31 Cloud 6. The mean spectrum is shown in the lower right panel. The source is only detected in the boresight beam. 


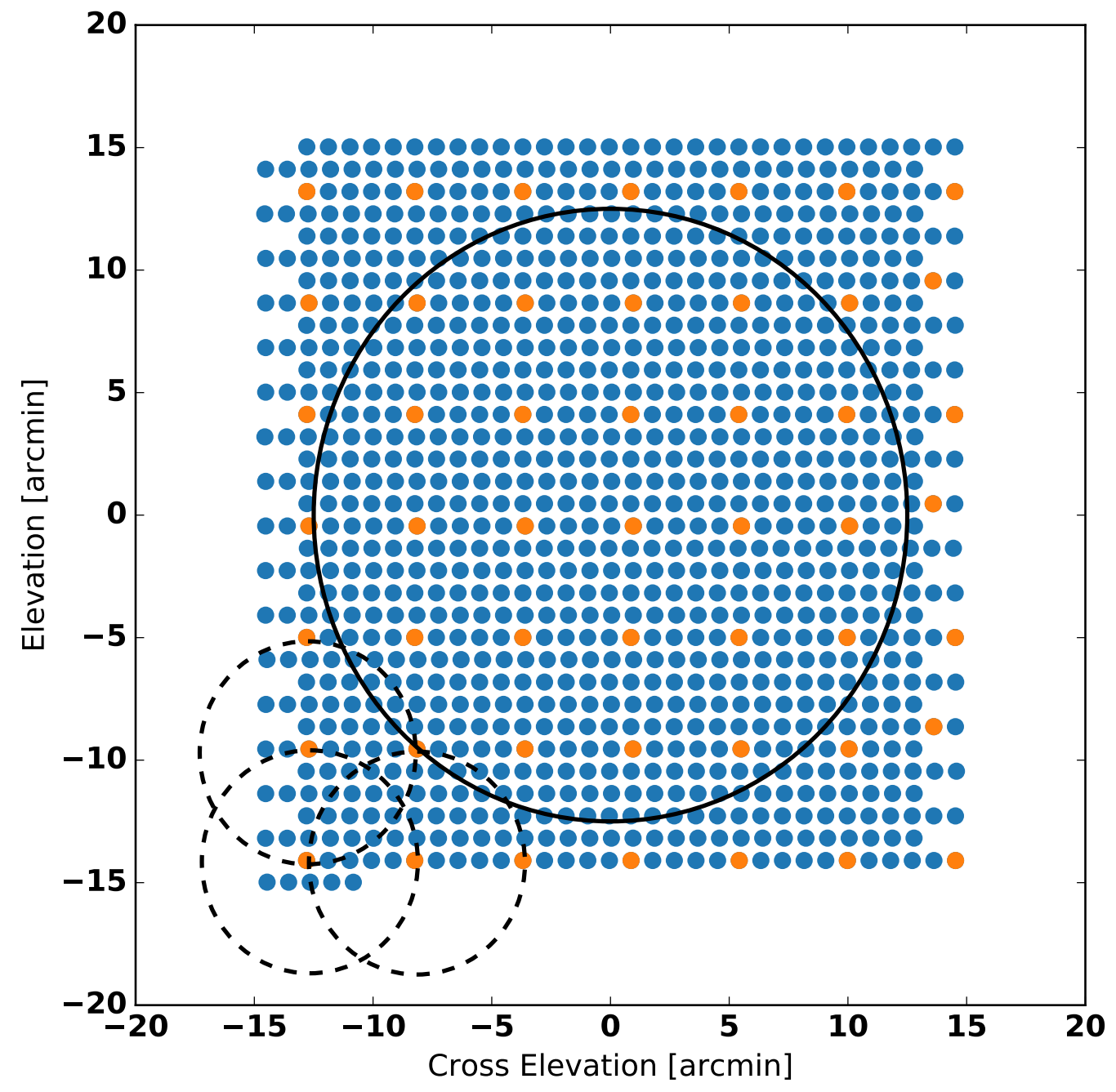

Figure 1.18 The formed beam locations over the PAF FOV (blue circles). The orange circles are the initial beam sample considered for the M31 Cloud 6 'snapshot' map based on the overlap at the half-power point. The orange outside the solid circle were excluded to ensure no loss of sensitivity. The dashed circles give an approximation of the angular widths of each beam. 

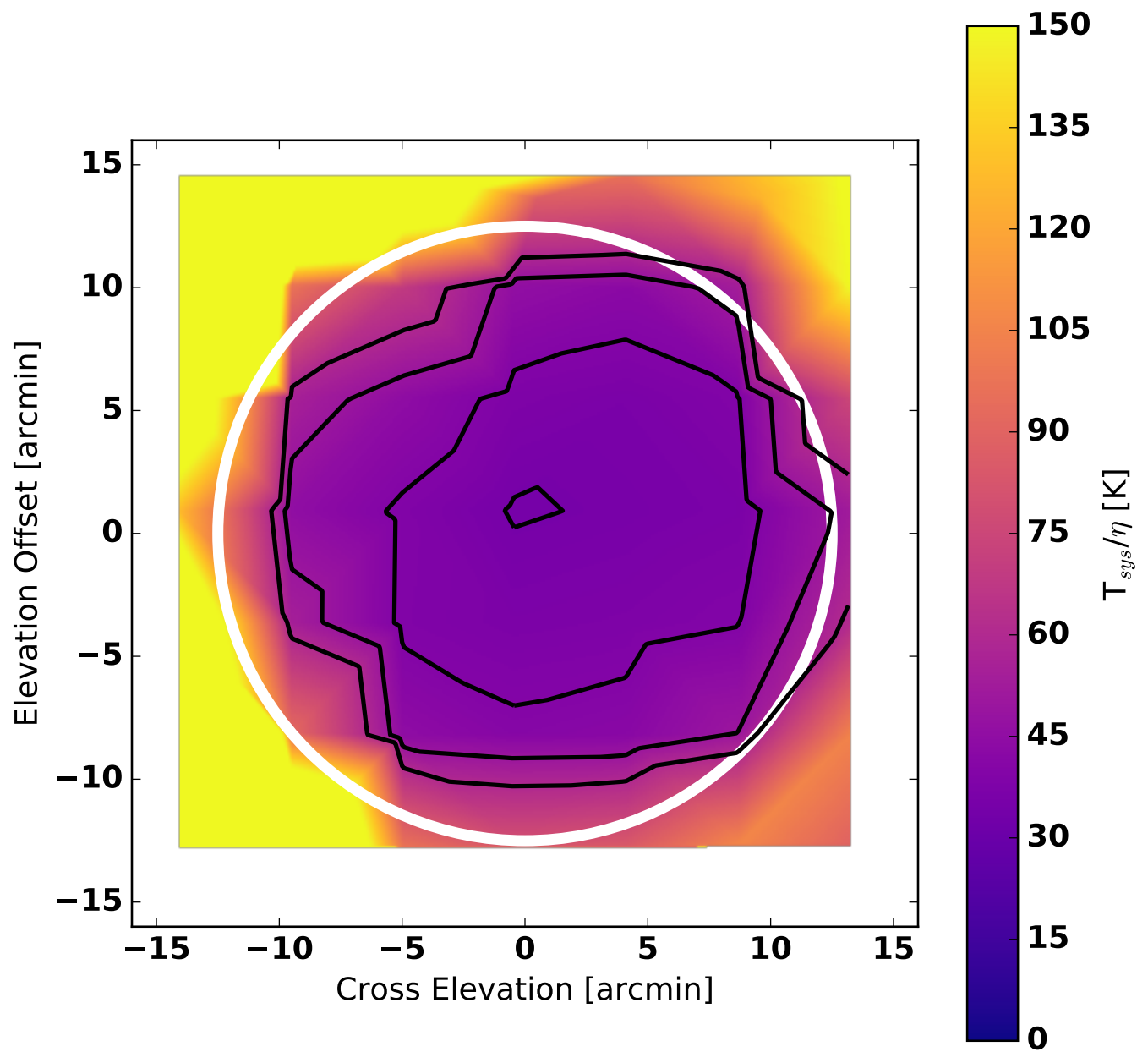

Figure 1.19 The $T_{\text {sys }} / \eta$ variations across the FOV for the snapshot image of M31 Cloud 6. The central contour level is $30 \mathrm{~K}$ and continues at $35,40,50$, and $60 \mathrm{~K}$. The thick white circle represents the angular threshold outside of which no formed beams were included in the snapshot image. 

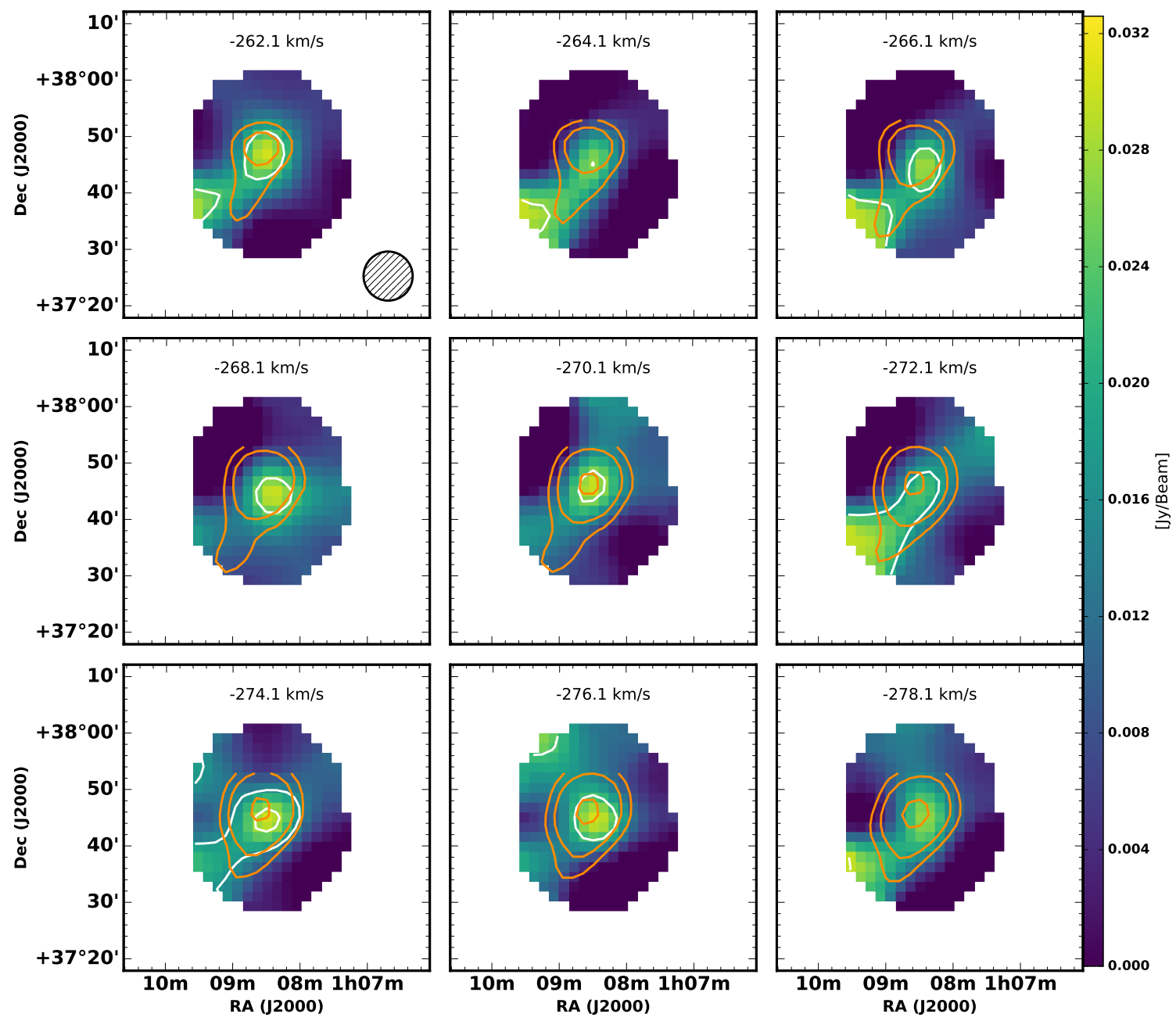

Figure 1.20 Channel maps of the M31 Cloud 6 Snapshot. In all panels, the color scale and white contours represent the FLAG data, while the orange contours are the single pixel counterpart data set; both sets of contours begin at the $3 \sigma$ noise of the FLAG data $(14 \mathrm{mK})$ and continue at 3, 5, and 10 times that level. 


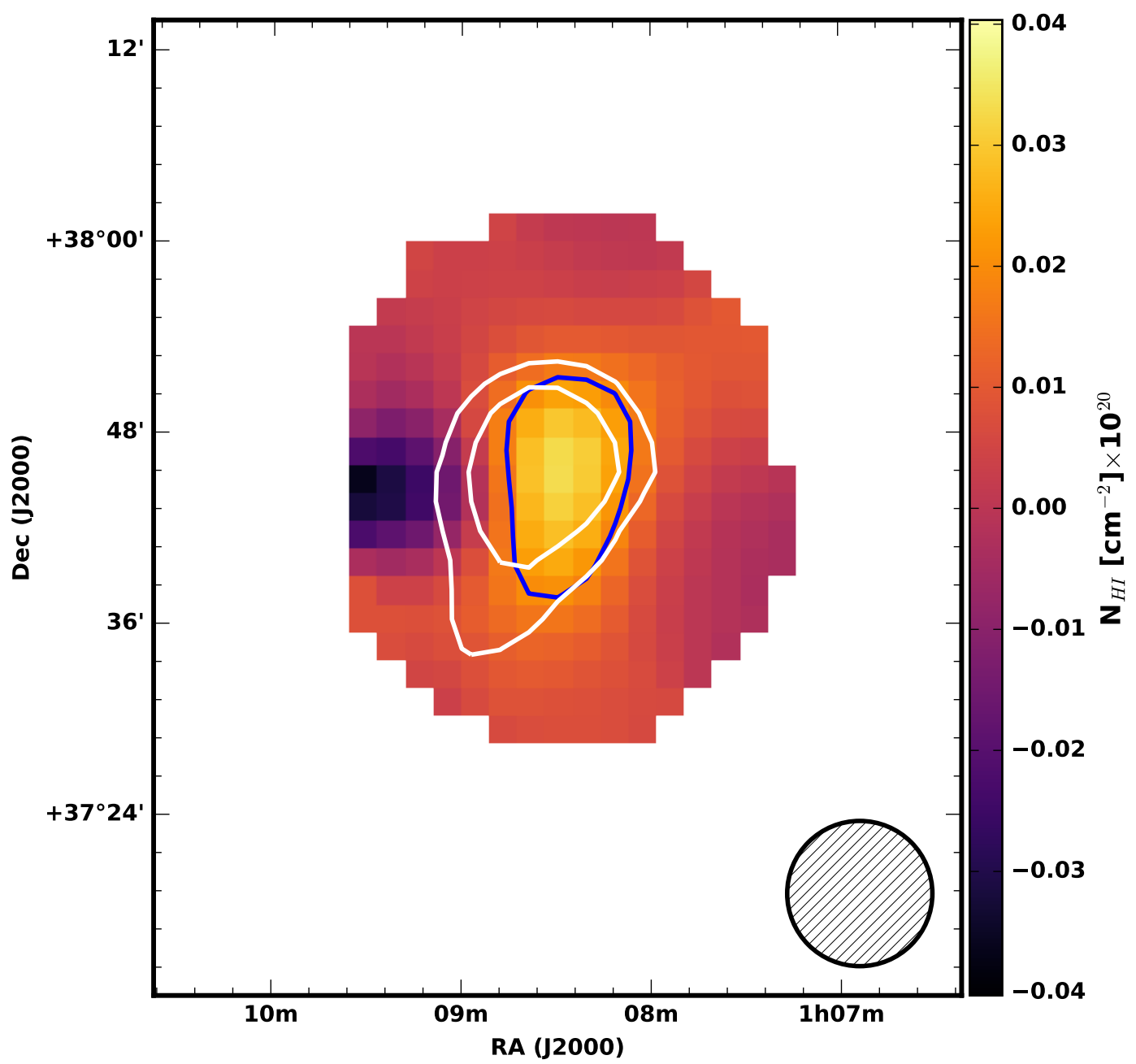

Figure 1.21 $N_{\mathrm{HI}}$ map of the M31 Cloud 6 Snapshot image. The color scale and blue contours represent the FLAG data, while the white contours are the single pixel counterpart data set; both sets of contours begin at the $3 \sigma$ of the FLAG data set $\left(2 \times 10^{18} \mathrm{~cm}^{-2}\right)$ and continue to 3 and 5 times that level. 
Figure 1.17 shows these calibrated spectra for each individually formed beam (blue) with the spectra taken from the location of the formed beam center in the single-pixel data cube (orange). The lower right panel shows the average spectrum of the seven formed beams. The source is only detected in the boresight beam, which is unsurprising given the half-power points of the formed beams were not overlapping during the 16B_400 observing run. The peak fluxes between the boresight spectrum and the single-pixel data match excellently. Additionally, the total flux detected in the boresight beam as determined by the integral between LSR velocities of -320 to $-245 \mathrm{~km} \mathrm{~s}^{-1}$ is $(1.60 \pm 0.13) \mathrm{Jy} \mathrm{km} \mathrm{s}^{-1}$, which matches the single pixel value of $(1.63 \pm 0.04) \mathrm{Jy} \mathrm{km} \mathrm{s}^{-1}$ within the statistical uncertainties.

There are some aspects of these spectra that emphasize issues with the system that need to be addressed in the future. For one, the apparent detection in Beam 3 is due to residual baseline structure. Even more pertinent are the artifacts caused by the scalloping that show up as spurious features seen in all individual beam spectra. Their presence explicitly demonstrates the necessity of implementing a revised PFB scheme in the PFBCORR mode to mitigate influence of the scalloping on the calibrated data. That said, the clear detection of M31 Cloud 6 in the boresight beam, and the excellent noise properties as compared to theoretical expectations further establishes the feasibility of using FLAG to study faint Hi sources at unprecedented levels.

Recall that correlation matrices characterizing the noise in the system and a signal are necessary for electronically forming a beam on the sky. A calibration grid procedure as described in Section consists of $\sim 1000$ 'signal' integrations over 
the $30 \times 30 \mathrm{deg}^{2}$ FOV traced out by the grid trajectory. In principle, if the nearest dedicated 'Off' pointing is used to obtain a noise correlation matrix, a beam can be formed at each of signal integrations resulting in a 'snapshot' of the sky intensity made with many hundreds of beams. Such an imaging technique would be beneficial for obtaining images of compact $\left(\lesssim 20^{\prime}\right)$, but still extended enough $\left(>9^{\prime}\right)$ to be resolved, sources within the FLAG FOV.

Beamformer weights were generated using the calibration grid of 3C147 for session GBT16B_400_14. Again, the spectra were calibrated according to Equations 4.9 and 1.10, but instead of seven total SDFITS files, a total of 1028 beams are available. The pointing centers of these beams are shown as circular blue points in Figure 1.18. Not all beams need to be used, however. The dashed circles in Figure 1.18 show the approximate beam width for several individual formed beams. The overlap from individual beams would lead to significant oversampling. The layout of the beams selected for imaging is such that the FOV has beams overlapping at their half-power points. The beams that fit this criteria are filled in with orange in Figure 1.18. There is still concern about the potentially steep drop in sensitivity towards the edge of FOV, which will contribute image artifacts near the edge of the FOV. The $T_{\text {sys }} / \eta$ for each beam is interpolated to a grid and shown in Figure 1.19. The variation across the image is moderately uniform within the central region up to about $12^{\prime}$ in radius before sharply increasing due to the loss response from dipoles that cannot see the point calibration source. To ensure the beams included in the final image do not suffer from degradation due to loss of sensitivity, only the beams within the spatial boundary denoted by the solid circles in Figures 1.18 (solid black) 
and 1.19 (solid white) are include in the final image. The data were imaged using the GBO pipeline task gbtgridder ${ }^{6}$ with a Gaussian-Bessel convolution function, $105 \square$ " pixels, with a spatial extent of $128 \times 128$ pixels.

Individual channel maps are shown in Figure 1.20. The channel maps show reasonable agreement between the contours of the FLAG and single-pixel data sets in most channels, although clear artifacts towards the edge of the FOV still have signatures that extend into the central region to affect the source emission. The $N_{\mathrm{HI}}$ image in Figure 1.21 reveals the artifact more clearly, indicating it is likely a product of degraded beamformer towards the edge of the FOV. That said, the contours tracing integrated emission from the two data sets match reasonably well at the faintest of $N_{\mathrm{HI}}$ levels. The generally good spatial agreement of the emission contours across multiple intensity levels over a diameter of $\sim 20^{\prime}$ demonstrates that this imaging mode has potential.

\subsection{Conclusions, Current Status and Future}

The spectral line commissioning results and derived system properties of a new beamformer for FLAG, a cryogenically cooled PAF for the GBT, were presented. The main conclusions from these initial data are:

- Custom software written to convert the raw correlation matrices to beamformed spectra that can be used in the GBO computing environment by the application of complex beam weights works as intended. The conversion, or 'fill-

\footnotetext{
${ }^{6}$ https://github.com/nrao/gbtgridder
} 
ing', software is available at https://github.com/nipingel/SpectralFiller.

- FLAG is most sensitive between the topocentric frequencies of $1200 \mathrm{MHz}$ up to about $1500 \mathrm{MHz}$ as revealed by the frequency sweep observation. The mean $T_{\text {sys }} / \eta$ derived for all beams over all observing runs is consistent with the existing single pixel L band receiver, agreeing with the conclusion of Roshi et al. (2018) that FLAG is currently the world's most sensitive PAF.

- On-the-fly maps of the external galaxy NGC6946 show equivalent $t_{\text {eff }}$ can be reached about seven times faster due to the increased FOV provided by FLAG over the single pixel receiver.

- Comparisons between FLAG maps and equivalent maps made with the singlepixel receiver show reasonable agreement in terms of the spatial contours of the emission - albeit nosier at the lowest statistically significant emission levels due to higher sidelobe levels in the formed beams. The source of the $\sim 30 \%$ flux offset in each beam as compared to equivalent single-pixel data still needs to be determined.

- The overall phase of the derived complex beamforming weight vectors does not vary much over timescales of $\sim 1$ week. However, the amplitude of the weights can vary largely between independent sessions on the timescale of days. The large spread in amplitude, while not detrimental to the overall sensitivity, will cause considerable gain variations across the FOV and introduce make flux scale matching between sessions. 
- A position switched observation of a faint Hi cloud reveals a clear detection in the boresight beam, verifying the capabilities of FLAG to probe low $N_{\mathrm{HI}}$ levels in extragalactic sources. An experimental snapshot image, where $\sim 45$ beams were simultaneously formed over the FOV of FLAG shows reasonable agreement with the single-pixel data both on a channel by channel comparison and for intensity integrated along the spectral axis. That said, due to a sharp decrease in sensitivity towards the edge of the FOV care must be taken to select the spatial layout of the beams; that is, only select beams that overlap at the half-power point within inner $\sim 20^{\prime}$ ). A snapshot imaging mode would be advantageous for acquiring images of objects smaller than about $20^{\prime}$ in extent, either through position switching or daisy mapping.

- Scalloping artifacts in fine channel mode, originating from the current implementation of the PFB, cause spurious spikes in the calibrated bandpass. While these can be mitigated by shifting the LO by one half of a coarse channel (i.e., $151.18 \mathrm{kHz}$ ) to create a frequency dithered image, the combination of these data introduces several additional calibration steps.

Given its status as the world's most sensitive PAF, FLAG will provide the GBT with a clear advantage over existing large single dish telescopes in terms of Hi survey speeds and help to ensure that it remains a premiere instrument for radio astrophysics. Open issues remain, however, that must be addressed to ensure reliable performance over observation sessions spread over multiple days. Firstly, the scalloping introduced by the current PFB implementation will be completely miti- 
gated in the next iteration of the backend software set to be written in the Summer of 2018. Secondly, individual threads handling one-twentieth of the bandpass simply die during a scan, which causes - in some cases - multiple missing chunks of spectra. These dropouts cause discontinuous spectra. The issue seems to be related to the efficiency of the current GPU codes and is a priority going forward. Finally, the loss of beamformer weight validity whenever the LO is changed due to loss of word/bit lock contributes to increased overhead. Good beamformer weights can be achieved within about 5 to 10 minutes through the use of 7 -point calibration scans, but it is still imperative that overhead is reduced

Although there are several moderate backend issues to be addressed before FLAG becomes a publicly available receiver for the GBT, the commissioning results presented in this chapter clearly establish it among the premiere PAFs currently in operation on large radio telescopes and primed to become a shared risk instrument. Results of the science mapping observations taken during Winter 2018 observing run (GBT17B_360, GBT17B_455, and GBT17B_360) to be presented in future publications show promising steps towards the reduction of overhead, observations of Galactic sources, and further sensitivity improvements. The future of FLAG and PAFs in general — to provide expedited mapping times of diffuse $N_{\mathrm{HI}}$ and is extremely promising. 


\section{Chapter 5}

\section{Conclusions}

\subsection{Final Conclusions}

The preceding chapters discussed results from deep Hi surveys performed with the GBT. The analysis of that data focused on probing the low Hi column density gas by investigating the fraction of diffuse Hi mass at $N_{\mathrm{HI}}$ levels below $10^{19} \mathrm{~cm}^{-2}$. The median mass fraction of less than $2 \%$ for this gas (compared to the total HI mass), coupled with the lack of diffuse features whose origin cannot be attributed to tidal forces, indicates the HI distribution in the HALOGAS galaxies does not differ significantly between the $N_{\mathrm{HI}}$ levels between and $10^{18} \mathrm{~cm}^{-2}$ and $10^{19} \mathrm{~cm}^{-2}$. In addition to a brief discussion on potential avenues for future analysis — specifically the combination of the GBT and WSRT in the Fourier plane to correct for missing short spacings — the final conclusions of this dissertation are summarized.

This work utilized deep Hi observations performed with the GBT to investigate the diffuse Hi associated with the HALOGAS sample of galaxies. The major method to probe the low column density Hi was the calculation of a parameter, $f_{19}$, that is effectively the ratio of the HI mass contained strictly at above a $N_{\mathrm{HI}}$ level of $10^{19}$ $\mathrm{cm}^{-2}$ to the total Hi mass. The major conclusions from this investigation are: (1) the median $f_{19}$ value is computed to be $0.022 \pm 0.002$, indicating that diffuse Hi reservoirs are not found to be prominent around the nearby galaxies of the HALOGAS sample; 
(2) two galaxies, NGC4258 and NGC4631, have distinctive diffuse Hi features that can be seen in individual channel maps and in the integrated Hi maps. While their $f_{19}$ values are relatively larger than the median at $0.065 \pm 0.003$ and $0.042 \pm 0.002$, respectively, the origin of these features are very likely tidal and not related in infalling cold gas from the IGM; (3) plotting $f_{19}$ verses several inherent galaxy properties that are predicted to be related to cold mode accretion — such as galaxy density and baryonic mass (Kereš et al., 2005) — revealed no trends.

Other results presented in this work were the commissioning Hi observations of a cyrogenically cooled PAF known as FLAG on the GBT that utilized a custom built beamforming backend. The custom reduction software, PAF_Filler.py, which performs the post-correlation beamforming given a set of complex beamforming weights, applies the beam offsets, and performs the necessary Doppler corrections, is successful in generating SDFITS files that can be manipulated in GBTIDL and reduced in commonly used radio astronomy software packages such as AIPS. Observations of NGC6946 successfully recreate the observed spatial distribution of the HI when compared with data taken with the current single-pixel receiver (Pisano, 2014). However, a flux offset on the order of $30 \%$ exists within these data, which can possibly be attributed to large variations in the scaling of the derived beamforming weights. A faint and compact Hi source, M31 Cloud 6, was observed in a positionswitched manner. This source is detected in the boresight beam of the PAF and agrees very well with on-the-fly mapping data from the single-pixel receiver (Wolfe et al., 2016) in terms of the overall profile shape and peak when the brightness temperature is plotted as a function of radial velocity. Since the calibration process 
of mapping over a bright calibrator results in many distinct beams over the $30 \times 30$ $\operatorname{arcminutes}^{2}$ area of the calibration map, a 'snapshot' of M31 Cloud 6 was attempted. This involved careful selection of the formed beams such that they overlap at the half-power points of their respective responses within the inherent FOV of FLAG (i.e. $<12.5^{\prime}$ in radius). While artifacts exist in the resulting images - likely from deceased sensitivity in the beams formed near the edge of the FOV — the relatively good agreement between the $N_{\mathrm{HI}}$ contours of the snapshot and single-pixel data is encouraging for the feasibility of this mode.

My plans for future work with these data include utilizing the GBT data to fill in the missing data in the center of the $u-v$ plane that is inherent to interferometer data will result in data cubes that combine the high angular resolution of the WSRT and superior angular sensitivity of the GBT. Given that the WSRT HALOGAS data are already the standard data sets for the search for cold gas accretion in the form of discrete Hi clouds, such combined cubes would form an excellent basis for future kinematic studies that identify infalling Hr. Such cubes could also be used to further probe the extreme outskirts of galaxy disks to characterize potential ionization edges as predicted by photoionization models. With a simple linear extrapolation of the available rotation curves of the HALOGAS galaxies, similar stacking experiments as those discussed in Ianjamasimanana et al. (2018) can be performed to increase the signal-to-noise of the already deep GBT data in search of a detection at previously unprobed $N_{\mathrm{HI}}$ levels. Such a detection would indicate there is HI at these low $N_{\mathrm{HI}}$ levels to be observed and bolster the case for the necessary time investment future HI surveys planned for the SKA and its precursors must make to detect Hi associated 
with the cosmic web. 


\section{Appendix A}

\section{Quantum Mechanical Derivation of the Spin-Flip Transition}

In a system consisting of two spin- $1 / 2$ particles, each particle can either be spin-up or spin-down; thus the four allowed basis sates are: $\uparrow \uparrow, \uparrow \downarrow, \downarrow \uparrow, \downarrow \downarrow$. In a more rigorous manner, these basis states can be defined defined as $\left|s_{1}, m_{1}\right\rangle \otimes\left|s_{2}, m_{2}\right\rangle$, where $s_{1}$ and $s_{2}$ are respectively the proton $\left(p^{+}\right)$and electron $\left(e^{-}\right)$spin states, and $m_{1}$ and $m_{2}$ are similarly the possible projections on the $z$-axis. The outer product in the above equation symbolizes that the coupled system spans a four dimensional Hilbert space.

Adding the angular momentum $s=s_{1}+s_{2}$ (sum of $p^{+}$and $e^{-}$spins), the coupled state is represented by

$$
|s, m\rangle=\sum_{m_{1}, m_{2}, m} C_{m_{1}, m_{2}, m}^{s_{1}, s_{2}, s}\left|s_{1}, m_{1}\right\rangle\left|s_{2}, m_{2}\right\rangle,
$$

where $C_{m_{1}, m_{2}, m}^{s_{1}, s_{2}, s}$ represents the expansion coefficients of total angular momentum, also known as Clebsch-Gordan coefficients (Griffiths, 1995). The basis vectors

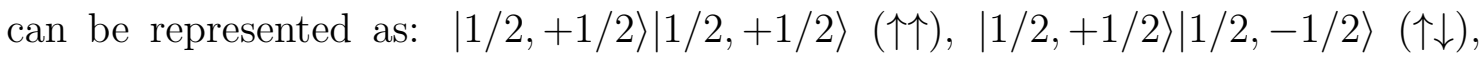
$|1 / 2,-1 / 2\rangle|1 / 2,+1 / 2\rangle(\downarrow \uparrow)$, and $|1 / 2,-1 / 2\rangle|1 / 2,-1 / 2\rangle(\downarrow \downarrow)$.

The sum of the angular momentum implies the system can only take on the 
values of either 0 or 1 . Thus, the three states with $s=1$ are

$$
\begin{gathered}
|1,1\rangle=|1 / 2,+1 / 2\rangle|1 / 2,+1 / 2\rangle=\uparrow \uparrow \\
|1,0\rangle=\frac{1}{\sqrt{2}}(|1 / 2,+1 / 2\rangle|1 / 2,-1 / 2\rangle+|1 / 2,-1 / 2\rangle|1 / 2,+1 / 2\rangle)=\frac{1}{\sqrt{2}}(\uparrow \downarrow+\downarrow \uparrow) \\
|1,-1\rangle=|1 / 2,-1 / 2\rangle|1 / 2,-1 / 2\rangle=\downarrow \downarrow
\end{gathered}
$$

and are known as the triplet states. The states where $s=0$, or the singlet state is

$$
|1,0\rangle=\frac{1}{\sqrt{2}}(|1 / 2,+1 / 2\rangle|1 / 2,-1 / 2\rangle-|1 / 2,-1 / 2\rangle|1 / 2,+1 / 2\rangle)=\frac{1}{\sqrt{2}}(\uparrow \downarrow-\downarrow \uparrow) .
$$

Due to the triplet states possessing the same total angular momentum, all three triplet states are degenerate in the absence of an external magnetic field. The energy difference between the triplet and singlet states arises from the alignment of the magnetic moments of the two particles.

A particle's magnetic moment $(\vec{\mu})$ is a quantity that determines the torque $(\tau=\vec{\mu} \times \vec{B})$ it will experienced in the presence of the magnetic field $\vec{B}$. The incremental work done by the field as the dipole is rotated through angle $d \theta$ is $d W=-\tau d \theta=-\mu B \sin \theta d \theta$. Knowing that the work done is equal to the decrease in potential energy: $d U=-d W=\mu B \sin \theta d \theta$, the potential energy is computed as such

$$
U=\int d U=\int|\mu||B| \sin \theta d \theta=-|\mu||B| \cos \theta+U_{0}=-\vec{\mu} \cdot \vec{B}
$$


where the integration constant of $U_{0}=0$ when choosing $U=0$ at $\theta=\pi / 2$.

Because the $p^{+}$and $e^{-}$each have equal but opposite charges, they each produce a magnetic field that generate a magnetic moment in the the other particle as they move relative to each other. It is these two interactions that ultimately generates the emission astronomers observe on Earth. Consider the interaction of two magnetic moments while the two radii of the $e^{-}$and $p^{+}$overlap. The form of the ground state wave function for hydrogen in spherical coordinates is

$$
\Psi_{1,0,0}(r, \theta, \phi)=\frac{1}{\sqrt{\pi a_{0}^{3}}} e^{-r / a_{0}}
$$

where $a_{0}=\frac{4 \pi \epsilon_{0} \hbar}{m q^{2}}$ is the Bohr radius (Griffiths, 1995). The probability of the $e^{-}$ being within the radius of the $p^{+}, b$, is therefore

$$
P(r \leq b)=\left\langle\Psi_{1,0,0}(r \leq b) \mid \Psi_{1,0,0}(r \leq b)\right\rangle=\frac{1}{\pi a_{0}^{3}} \int_{0}^{2 \pi} \int_{0}^{\pi} \int_{0}^{b} e^{-2 r / a_{0}} r^{2} \sin \theta d r d \theta d \phi
$$

Integrating by parts twice gives

$$
P(r \leq b)=1-e^{2 b / a}\left(1+2 b / a_{0}+2\left(b / a_{0}\right)^{2}\right),
$$

which reduces to $P(r \leq b)=\xi / 6$, or $4 b^{3} / 3 a_{0}^{3}$ when taking the lowest non-trivial term in the Taylor expansion of Equation A.9 with $2 b / a_{0}$ set to $\xi$.

To calculate the energy difference between the triplet and singlet energy states when the magnetic moment $\mu_{e}$ of the $e^{-}$interacts with the magnetic field of the 
proton $B_{p}$, combine the approximated result of Equation A.9 with that of A.6

$$
U=-P\left(\overrightarrow{\mu_{e}} \cdot \overrightarrow{B_{p}}\right)
$$

Knowing that the magnetic moment of an $e^{-}$is

$$
\overrightarrow{\mu_{e}}=g_{e} \frac{q}{2 m_{e}} \overrightarrow{S_{e}}
$$

where $\vec{S}_{e}$ is the spin state and $g_{e}$ is the g-factor (a quantity that characterizes the magnetic moment and gyromagnetic ratio) equal to -2.002319 (Mohr et al., 2016), and $m_{e}$ is the mass of an electron, one can calculate the potential energy of the spin interaction in the presence of a directed external magnetic field. The magnetic moment of a $p^{+}$is similarly defined as $\overrightarrow{\mu_{p}}=g_{p} \frac{q}{2 m_{p}} \vec{S}$, where $g_{p}, \overrightarrow{S_{p}} m_{p}$ represent the g-factor of 5.58569 (Mohr et al., 2016), the spin state, and mass of a proton.

Assuming the magnetic field inside the spherical $p^{+}$is passing uniformly through, it can be approximated as $\overrightarrow{B_{p}}=\frac{\mu_{0}}{2 \pi b^{3}} \cdot \overrightarrow{\mu_{p}}$ (see Application 13.1 in Zangwill 2013). Applying Equation A.10, the potential energy is explicitly given by

$$
U=\frac{\mu_{0} g_{e} g_{p} e^{2}}{3 a_{0}^{3} \pi m_{p} m_{e}} \overrightarrow{S_{p}} \cdot \overrightarrow{S_{e}}
$$

The operator, $\overrightarrow{S_{p}} \cdot \overrightarrow{S_{e}}$ (Griffiths, 1995), is the spin-spin coupling factor, which fundamentally demonstrates the origin of of the energy difference between the triplet and singlet eigenstates. To explicitly calculate the difference in potential energies, take 
the dot product of the total angular momentum operator $\vec{S} \cdot \vec{S}=\left(\overrightarrow{S_{e}}+\overrightarrow{S_{p}}\right) \cdot\left(\overrightarrow{S_{e}}+\overrightarrow{S_{p}}\right)$ with itself, and rearrange to find

$$
\overrightarrow{S_{e}} \cdot \overrightarrow{S_{p}}=\frac{1}{2}\left(\vec{S} \cdot \vec{S}-\overrightarrow{S_{e}} \cdot \overrightarrow{S_{e}}-\overrightarrow{S_{p}} \cdot \overrightarrow{S_{p}}\right)
$$

which is an eigenvalues equation that explicitly relates the total energy of an eigenstate to the total angular momentum, $\ell=0$ or $1, p^{+}$spin $s_{1}=1 / 2$ and $-1 / 2$, and similarly $e^{-}$spin $s_{2}$. Calculating Equation A.13 for the triplet state where $\ell$ $=1, s_{1}=1 / 2$, and $s_{2}=1 / 2$ gives $\frac{1}{4} \hbar^{2}|1,1\rangle$. The operator applied to the singlet state gives $-\frac{3}{4} \hbar^{2}|0,0\rangle$. These values demonstrate that the an Hi atom is in a higher energy state when the the two constituent particle spins are aligned than when they are anti-aligned in the singlet state. Knowing these values, and utilizing Equation A.12, the difference in potential energy is therefore $9.411718 \times 10^{-18}$ erg; thus, when an Hi atom spontaneously decays from the state where the spins are aligned (but the magnetic moments are anti-aligned due to the oppositely charged particles), a photon is released with a rest frame frequency

$$
\nu_{H I}=\frac{E}{h}=\frac{9.411718 \times 10^{-18} \mathrm{erg}}{6.626070 \times 10^{-27} \mathrm{erg} \mathrm{s}}=1420.405751 \mathrm{MHz}
$$

where $h$ is the Planck constant. This is the very frequency predicted by van de Hulst in 1944 and later observed by Ewen \& Purcell (1951). 


\section{Bibliography}

Aniano, G., Draine, B. T., Gordon, K. D., \& Sandstrom, K. 2011, PASP, 123, 1218

Bannister, K. W., Shannon, R. M., Macquart, J.-P., et al. 2017, ApJL, 841, L12

Barnes, D. G., Staveley-Smith, L., de Blok, W. J. G., et al. 2001, MNRAS, 322, 486

Begeman, K. G. 1989, A\&A, 223, 47

Bekki, K. 2008, MNRAS, 390, L24

Benjamin, R. A. 2002, in Astronomical Society of the Pacific Conference Series, Vol. 276, Seeing Through the Dust: The Detection of HI and the Exploration of the ISM in Galaxies, ed. A. R. Taylor, T. L. Landecker, \& A. G. Willis, 201

Birnboim, Y., \& Dekel, A. 2003, MNRAS, 345, 349

Bland-Hawthorn, J., Maloney, P. R., Stephens, A., Zovaro, A., \& Popping, A. 2017, ApJ, 849, 51

Boomsma, R., Oosterloo, T. A., Fraternali, F., van der Hulst, J. M., \& Sancisi, R. 2008, A\&A, 490, 555

Boothroyd, A. I., Blagrave, K., Lockman, F. J., et al. 2011, A\&A, 536, A81

Bosma, A. 1983, in IAU Symposium, Vol. 100, Internal Kinematics and Dynamics of Galaxies, ed. E. Athanassoula, 11-20 
Braun, R., \& Thilker, D. 2004, in Astronomical Society of the Pacific Conference Series, Vol. 327, Satellites and Tidal Streams, ed. F. Prada, D. Martinez Delgado, \& T. J. Mahoney, 139

Braun, R., \& Walterbos, R. A. M. 1985, A\&A, 143, 307

Bregman, J. N. 1980, ApJ, 236, 577

Buat, V., Boissier, S., Burgarella, D., et al. 2008, A\&A, 483, 107

Buat, V., Giovannoli, E., Heinis, S., et al. 2011, A\&A, 533, A93

Chynoweth, K. M., Langston, G. I., \& Holley-Bockelmann, K. 2011, AJ, 141, 9

Cluver, M. E., Jarrett, T. H., Hopkins, A. M., et al. 2014, ApJ, 782, 90

Combes, F. 1978, A\&A, 65, 47

Combes, F., Foy, F. C., Gottesman, S. T., \& Weliachew, L. 1980, A\&A, 84, 85

Condon, J. J., \& Ransom, S. M. 2016, Essential Radio Astronomy

Corbelli, E., Lorenzoni, S., Walterbos, R., Braun, R., \& Thilker, D. 2010, A\&A, 511, A89

Cox, A. L., Sparke, L. S., van Moorsel, G., \& Shaw, M. 1996, AJ, 111, 1505

Dahari, O. A. 1984, PhD thesis, California Univ., Santa Cruz.

de Blok, W. J. G., Keating, K. M., Pisano, D. J., et al. 2014a, A\&A, 569, A68

de Blok, W. J. G., Józsa, G. I. G., Patterson, M., et al. 2014b, A\&A, 566, A80 
de Blok, W. J. G., Adams, E. A. K., Amram, P., et al. 2017, ArXiv e-prints, arXiv:1709.08458

de Vaucouleurs, G., de Vaucouleurs, A., \& Corwin, J. R. 1976, in Second reference catalogue of bright galaxies, Vol. 1976, p. Austin: University of Texas Press., Vol. 1976

DeBoer, D. R., Gough, R. G., Bunton, J. D., et al. 2009, IEEE Proceedings, 97, 1507

Di Teodoro, E. M., McClure-Griffiths, N. M., Lockman, F. J., et al. 2018, ApJ, 855, 33

Dove, J. B., \& Shull, J. M. 1994, ApJ, 423, 196

Driver, S. P., Norberg, P., Baldry, I. K., et al. 2009, Astronomy and Geophysics, $50,5.12$

Driver, S. P., Hill, D. T., Kelvin, L. S., et al. 2011, MNRAS, 413, 971

Elmer, M., Jeffs, B. D., Warnick, K. F., Fisher, J. R., \& Norrod, R. D. 2012, IEEE Transactions on Antennas and Propagation, 60, 903

Eneev, T. M., Kozlov, N. N., \& Sunyaev, R. A. 1973, A\&A, 22, 41

Ewen, H. I., \& Purcell, E. M. 1951, Nature, 168, 356

Fisher, J. R., \& Bradley, R. F. 2000, in Proc. SPIE, Vol. 4015, Radio Telescopes, ed. H. R. Butcher, 308-318 
Fraternali, F. 2017, in Astrophysics and Space Science Library, Vol. 430, Gas Accretion onto Galaxies, ed. A. Fox \& R. Davé, 323

Fraternali, F., \& Binney, J. J. 2006, MNRAS, 366, 449

—. 2008, MNRAS, 386, 935

Fraternali, F., Marasco, A., Armillotta, L., \& Marinacci, F. 2015, MNRAS, 447, L70

Fraternali, F., Oosterloo, T., Sancisi, R., \& van Moorsel, G. 2001, ApJL, 562, L47

Fraternali, F., van Moorsel, G., Sancisi, R., \& Oosterloo, T. 2002, AJ, 123, 3124

Frenk, C. S., White, S. D. M., Efstathiou, G., \& Davis, M. 1985, Nature, 317, 595

García-Ruiz, I., Sancisi, R., \& Kuijken, K. 2002, A\&A, 394, 769

Gil de Paz, A., Boissier, S., Madore, B. F., et al. 2007, ApJS, 173, 185

Gottesman, S. T. 1980, AJ, 85, 824

Griffiths, D. J. 1995, Introduction to Quantum Mechanics

Haynes, M. P., \& ALFALFA Team. 2008, in American Institute of Physics Conference Series, Vol. 1035, The Evolution of Galaxies Through the Neutral Hydrogen Window, ed. R. Minchin \& E. Momjian, 238-241

Haynes, M. P., Giovanelli, R., Martin, A. M., et al. 2011, AJ, 142, 170

Heald, G., Józsa, G., Serra, P., et al. 2011, A\&A, 526, A118 
-. 2012, A\&A, 544, C1

Heywood, I., Bannister, K. W., Marvil, J., et al. 2016, MNRAS, 457, 4160

Hobbs, G., Heywood, I., Bell, M. E., et al. 2016, MNRAS, 456, 3948

Ianjamasimanana, R., de Blok, W. J. G., \& Heald, G. H. 2017, AJ, 153, 213

Ianjamasimanana, R., Walter, F., de Blok, W. J. G., Heald, G. H., \& Brinks, E. 2018, ArXiv e-prints, arXiv:1803.10291

Jarrett, T. H., Masci, F., Tsai, C. W., et al. 2013, AJ, 145, 6

Jeffs, B. D., Warnick, K. F., Landon, J., et al. 2008, IEEE Journal of Selected Topics in Signal Processing, 2, 635

Jorsater, S., \& van Moorsel, G. A. 1995, AJ, 110, 2037

Joung, M. R., Bryan, G. L., \& Putman, M. E. 2012, ApJ, 745, 148

Kamphuis, P., Rand, R. J., Józsa, G. I. G., et al. 2013, MNRAS, 434, 2069

Kannappan, S. J. 2004, ApJL, 611, L89

Kannappan, S. J., Stark, D. V., Eckert, K. D., et al. 2013, ApJ, 777, 42

Karachentsev, I. D., \& Kaisina, E. I. 2013, AJ, 146, 46

Kauffmann, G., Li, C., \& Heckman, T. M. 2010, MNRAS, 409, 491

Kemble, E. C., \& Zener, C. 1929, Physical Review, 33, 512 
Kereš, D., Katz, N., Fardal, M., Davé, R., \& Weinberg, D. H. 2009, MNRAS, 395, 160

Kereš, D., Katz, N., Weinberg, D. H., \& Davé, R. 2005, MNRAS, 363, 2

Landon, J., Elmer, M., Waldron, J., et al. 2010, AJ, 139, 1154

Lelli, F., Verheijen, M., \& Fraternali, F. 2014, MNRAS, 445, 1694

Madau, P., \& Dickinson, M. 2014, ARA\&A, 52, 415

Maloney, P. 1993, ApJ, 414, 41

Mangum, J. G., Emerson, D. T., \& Greisen, E. W. 2007, A\&A, 474, 679

Marinacci, F., Fraternali, F., Ciotti, L., \& Nipoti, C. 2010, MNRAS, 401, 2451

Marinacci, F., Grand, R. J. J., Pakmor, R., et al. 2017, MNRAS, 466, 3859

Mohr, P. J., Newell, D. B., \& Taylor, B. N. 2016, Reviews of Modern Physics, 88, 035009

Morgan, M. A., Fisher, J. R., \& Castro, J. J. 2013, PASP, 125, 695

Moss, V. A., McClure-Griffiths, N. M., Murphy, T., et al. 2013, ApJS, 209, 12

Nelson, D., Vogelsberger, M., Genel, S., et al. 2013, MNRAS, 429, 3353

Norman, C. A., \& Ikeuchi, S. 1989, ApJ, 345, 372

Noterdaeme, P., Petitjean, P., Carithers, W. C., et al. 2012, A\&A, 547, L1

Nuza, S. E., Parisi, F., Scannapieco, C., et al. 2014, MNRAS, 441, 2593 
Ocvirk, P., Pichon, C., \& Teyssier, R. 2008, MNRAS, 390, 1326

Oosterloo, T., Fraternali, F., \& Sancisi, R. 2007, AJ, 134, 1019

Oosterloo, T., Verheijen, M. A. W., van Cappellen, W., et al. 2009, in Wide Field Astronomy Technology for the Square Kilometre Array, 70

Perley, R. A., \& Butler, B. J. 2017, ApJS, 230, 7

Pingel, N., Lee, M.-Y., Burkhart, B., \& Stanimirović, S. 2018, ArXiv e-prints, arXiv:1802.10092

Pingel, N. M., Stanimirović, S., Peek, J. E. G., et al. 2013, ApJ, 779, 36

Pisano, D. J. 2014, AJ, 147, 48

Pisano, D. J., Wilcots, E. M., \& Elmegreen, B. G. 1998, AJ, 115, 975

Popping, A., Davé, R., Braun, R., \& Oppenheimer, B. D. 2009, A\&A, 504, 15

Prialnik, D. 2009, An Introduction to the Theory of Stellar Structure and Evolution

Putman, M. E. 2000, PASA, 17, 1

Putman, M. E., Peek, J. E. G., Muratov, A., et al. 2009, ApJ, 703, 1486

Rahmati, A., Schaye, J., Bower, R. G., et al. 2015, MNRAS, 452, 2034

Rand, R. J. 1994, A\&A, 285, 833

Rand, R. J., Allan, J., Heald, G., \& Jozsa, G. 2011, in Bulletin of the American Astronomical Society, Vol. 43, American Astronomical Society Meeting Abstracts \#217, 246.20 
Rees, M. J., \& Ostriker, J. P. 1977, MNRAS, 179, 541

Reynolds, T. N., Staveley-Smith, L., Rhee, J., et al. 2017, PASA, 34, e051

Rhee, J., Lah, P., Briggs, F. H., et al. 2018, MNRAS, 473, 1879

Ribaudo, J., Lehner, N., Howk, J. C., et al. 2011, ApJ, 743, 207

Richter, P., Nuza, S. E., Fox, A. J., et al. 2017, A\&A, 607, A48

Roberts, M. S. 1966, Physical Review Letters, 17, 1203

Roshi, A. D., Shillue, W., Simon, B., et al. 2018, ArXiv e-prints, arXiv:1803.04473

Rupen, M. P. 1991, AJ, 102, 48

Sancisi, R., \& Allen, R. J. 1979, A\&A, 74, 73

Sancisi, R., Fraternali, F., Oosterloo, T., \& van der Hulst, T. 2008, A\&A Rev., 15, 189

Sault, R. J., Teuben, P. J., \& Wright, M. C. H. 1995, in Astronomical Society of the Pacific Conference Series, Vol. 77, Astronomical Data Analysis Software and Systems IV, ed. R. A. Shaw, H. E. Payne, \& J. J. E. Hayes, 433

Sohn, Y.-J., \& Davidge, T. J. 1996, AJ, 112, 2559

Stanimirovic, S. 2002, in Astronomical Society of the Pacific Conference Series, Vol. 278, Single-Dish Radio Astronomy: Techniques and Applications, ed. S. Stanimirovic, D. Altschuler, P. Goldsmith, \& C. Salter, 375-396 
Stanimirović, S., Staveley-Smith, L., Dickey, J. M., Sault, R. J., \& Snowden, S. L. 1999, MNRAS, 302, 417

Stil, J. M., \& Israel, F. P. 2002, A\&A, 389, 29

Stocke, J. T., Keeney, B. A., \& Danforth, C. W. 2010, PASA, 27, 256

Swaters, R. A., Sancisi, R., \& van der Hulst, J. M. 1997, ApJ, 491, 140

Tully, R. B. 1988, Nearby galaxies catalog

van der Kruit, P. C., Oort, J. H., \& Mathewson, D. S. 1972, A\&A, 21, 169

van Gorkom, J. 1996, in Astronomical Society of the Pacific Conference Series, Vol. 106, The Minnesota Lectures on Extragalactic Neutral Hydrogen, ed. E. D. Skillman, 293

Vargas, C. J., Heald, G., Walterbos, R. A. M., et al. 2017, ApJ, 839, 118

Verheijen, M. A. W., \& Sancisi, R. 2001, A\&A, 370, 765

Vilnrotter, V., Fort, D., \& Iijima, B. 1995, in Astronomical Society of the Pacific Conference Series, Vol. 75, Multi-Feed Systems for Radio Telescopes, ed. D. T. Emerson \& J. M. Payne, 61

Wakker, B. P., Savage, B. D., Sembach, K. R., et al. 2003, ApJS, 146, 1

Wallin, J. F., Holincheck, A. J., \& Harvey, A. 2016, Astronomy and Computing, 16, 26

Walter, F., Brinks, E., de Blok, W. J. G., et al. 2008, AJ, 136, 2563 
Weliachew, L., Sancisi, R., \& Guelin, M. 1978, A\&A, 65, 37

White, S. D. M., \& Frenk, C. S. 1991, ApJ, 379, 52

Wilson, T. L., Rohlfs, K., \& Hüttemeister, S. 2013, Tools of Radio Astronomy, doi:10.1007/978-3-642-39950-3

Wolfe, S. A., Lockman, F. J., \& Pisano, D. J. 2016, ApJ, 816, 81

Wolfe, S. A., Pisano, D. J., \& Lockman, F. J. 2015, GBT Memo Series, GBT Memo 289

Wolfe, S. A., Pisano, D. J., Lockman, F. J., McGaugh, S. S., \& Shaya, E. J. 2013, Nature, 497, 224

Wolfinger, K., Kilborn, V. A., Koribalski, B. S., et al. 2013, MNRAS, 428, 1790

Zangwill, A. 2013, Modern Electrodynamics

Zhang, H.-X., Hunter, D. A., \& Elmegreen, B. G. 2012, ApJ, 754, 29

Zhu, Y.-N., Wu, H., Li, H.-N., \& Cao, C. 2010, Research in Astronomy and Astrophysics, 10, 329

Zschaechner, L. K., Rand, R. J., Heald, G. H., Gentile, G., \& Józsa, G. 2012, ApJ, 760,37

Zschaechner, L. K., Rand, R. J., Heald, G. H., Gentile, G., \& Kamphuis, P. 2011, ArXiv e-prints, arXiv:1107.4064 